\title{
Vascular and renal adjustments during and after preeclampsia
}

Citation for published version (APA):

Lopes van Balen, V. A. (2018). Vascular and renal adjustments during and after preeclampsia. [Doctoral Thesis, Maastricht University]. Maastricht University. https://doi.org/10.26481/dis.20181102vl

Document status and date:

Published: 01/01/2018

DOI:

10.26481/dis.20181102vl

Document Version:

Publisher's PDF, also known as Version of record

\section{Please check the document version of this publication:}

- A submitted manuscript is the version of the article upon submission and before peer-review. There can be important differences between the submitted version and the official published version of record.

People interested in the research are advised to contact the author for the final version of the publication, or visit the DOI to the publisher's website.

- The final author version and the galley proof are versions of the publication after peer review.

- The final published version features the final layout of the paper including the volume, issue and page numbers.

Link to publication

\footnotetext{
General rights rights.

- You may freely distribute the URL identifying the publication in the public portal. please follow below link for the End User Agreement:

www.umlib.nl/taverne-license

Take down policy

If you believe that this document breaches copyright please contact us at:

repository@maastrichtuniversity.nl

providing details and we will investigate your claim.
}

Copyright and moral rights for the publications made accessible in the public portal are retained by the authors and/or other copyright owners and it is a condition of accessing publications that users recognise and abide by the legal requirements associated with these

- Users may download and print one copy of any publication from the public portal for the purpose of private study or research.

- You may not further distribute the material or use it for any profit-making activity or commercial gain

If the publication is distributed under the terms of Article $25 \mathrm{fa}$ of the Dutch Copyright Act, indicated by the "Taverne" license above, 


\section{VASCULAR AND RENAL ADJUSTMENTS DURING AND AFTER PREECLAMPSIA}


Financial support by Mitch \& Mates for the publication of this thesis is gratefully acknowledged.

\section{mitch\&mates}

Financial support by the Dutch Heart Foundation for the publication of this thesis is gratefully acknowledged.

Financial support by the Dutch Kidney Foundation for the publication of this thesis is gratefully acknowledged.

ISBN:

978-94-028-1144-5

Cover design and layout:

(c) evelienjagtman.com

Print:

Ipskamp printing, ww.proefschriften.net

(c) V.A. Lopes van Balen, Maastricht 2018

All rights are reserved. No part of this book may be reproduced or distributed in any form or by any means, without prior written permission of the author. 


\title{
VASCULAR AND RENAL ADJUSTMENTS \\ DURING AND AFTER PREECLAMPSIA
}

\author{
Proefschrift \\ ter verkrijging van de graad van doctor \\ aan de Universiteit van Maastricht, \\ op gezag van Rector Magnificus, Prof. dr. Rianne M. Letschert, \\ volgens het besluit van het college van Decanen, \\ in het openbaar te verdedigen op \\ 02-11-2018 om 12:00 uur
}

door

Veronica Agatha Lopes van Balen

geboren op 23-02-1987

te Rio de Janeiro 


\section{Promotor}

Prof. dr. M.E.A. Spaanderman

\section{Copromotor}

Dr. J.J. Spaan, Amphia Ziekenhuis, Breda

\section{Beoordelingscommissie}

Prof. dr. B.W.W. Kramer (voorzitter)

Prof. dr. W. Gyselaers, Ziekenhuis Oost-Limburg, Genk, België

Prof. dr. M.T.E. Hopman, Radboud Universiteit Nijmegen

Prof. dr. J.P. Kooman 


\section{TABLE OF CONTENTS}

\section{CHAPTER 1 General}

introduction

CHAPTER 2

Physiologic adaptation of endothelial function to pregnancy:

a systematic review and meta-analysis

\section{CHAPTER 3}

Kidney function during pregnancy: a systematic review and meta-analysis

\section{CHAPTER 4}

Early pregnancy circulatory adaptation and recurrent hypertensive

disease: an explorative study

\section{CHAPTER 5}

Prevalence of chronic kidney disease after preeclampsia

\section{CHAPTER 6}

Endothelial and kidney function in women with a history of preeclampsia

and healthy parous controls

\section{CHAPTER 7}

Vascular aging in young and middle-aged women after a hypertensive complicated and uncomplicated pregnancy

\section{CHAPTER 8}

General discussion

\section{CHAPTER 9}

Valorisation

\section{CHAPTER 10}

Summary/Samenvatting

CHAPTER 11

List of co-authors / List of publications / Curriculum Vitae / Dankwoord 

Para Regina Maria Lopes van Balen Minha querida amiga e mãe 


\section{CHAPTER 1}

General introduction 



\section{INTRODUCTION}

Our endothelium serves as a distribution network that interacts with all organs and plays a part in several regulatory vessel functions. Endothelial cells respond to the specific environment they are in and vascular endothelial dysfunction can therefore express itself in different ways. Research regarding expressions of endothelial function during pregnancy, such as vasodilation and kidney function, can provide more insight in maternal adaptation to pregnancy. Moreover, pregnancies complicated by a vascular hypertensive disease affect endothelial function and are a window into future cardiovascular and kidney disease.

\section{OUR ENDOTHELIUM}

Endothelium is a monocellular layer present at the inner surface of blood vessels, separating circulating blood from tissue. It responds to physical and/or chemical stimuli with the release of corrective paracrine and endocrine substances to maintain vasomotor balance, vascular-tissue integrity and blood homeostasis. Therefore, it can be considered the largest endocrine organ in the human body. Different mechanisms that are kept in balance by the endothelium are vaso- constriction and dilation, thrombolysis and thrombosis, anti-proliferation and growth factors, (anti) inflammation, (anti) oxidation, platelet-disaggregation and adhesion (see Figure 1) ${ }^{1}$.

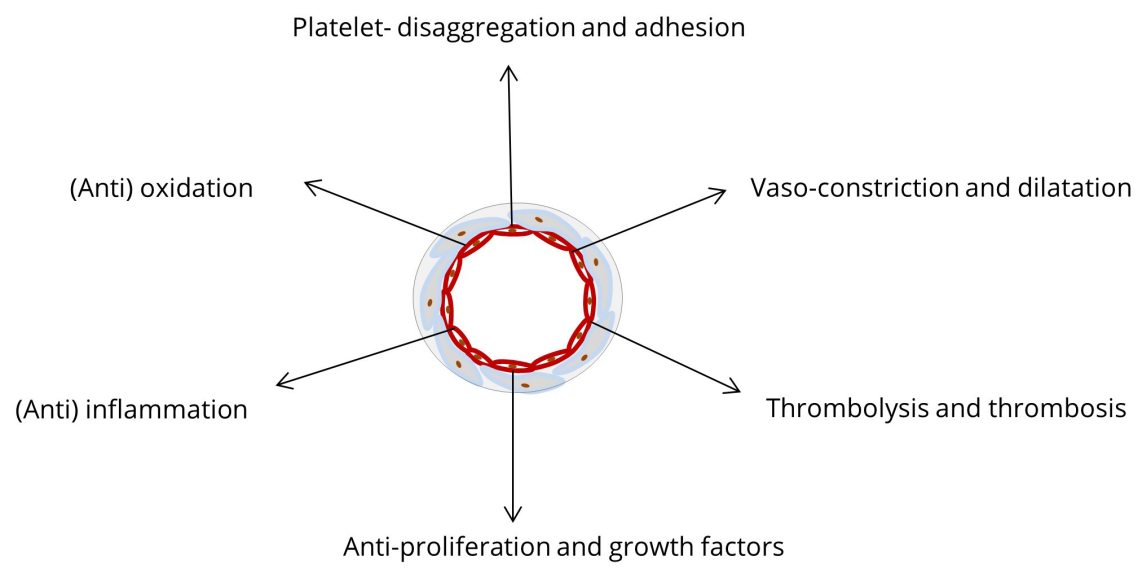

Figure 1 Schematic cross sectional representation of a blood vessel and the functions of our endothelium. 
The vaso-homeostasis is important in regulating (blood) pressure and in the reduction of stress on the vessel wall. It is primarily effectuated by nitric oxide (NO), prostacyclin, endothelium derived hyperpolarizing factor, angiotensin, endothelin, thromboxane and an extensive network of feedback stimuli 2,3 . NO, the most prominent effector, is primarily released as a consequence of increased shear stress caused by alterations in (blood) flow. Interestingly, the endothelial cell itself adapts its shape based on the mechanical forces of blood flow and distension, this in order to reduce the occurrence of vascular lesions ${ }^{1}$.

\section{WHY IS OUR ENDOTHELIUM INTERESTING?}

A dysfunctioning endothelium, resulting in a dysbalance of excreted substances, is considered the first step in the development of atherosclerosis and subsequently cardiovascular diseases (CVD). When the endothelium loses its ability to maintain the delicate balance of mechanical and biochemical vaso-homeostasis, it becomes vulnerable to the invasion of lipids and leukocytes at locations where lesions occur.

Atherosclerosis may begin as early as in childhood with the development of fatty streaks inside small lesions ${ }^{4}$. Over time a fatty streak can develop into a plaque. The advanced lesions of atherosclerosis occur with increasing frequency with age and this process can be accelerated by (classical) cardiovascular risk factors. Classical, but also modifiable, risk factors are diabetes mellitus, kidney disease, dyslipidaemia, obesity, smoking, alcohol intake, lack of exercise and cafeteria diet. Classical non-modifiable risk factors are family history, gender and age ${ }^{5}$. Other factors that are thought to negatively affect endothelial function are: an increase in endogenous endothelial NO synthase (eNOS) inhibitor, raised levels of vasoconstrictors such as angiotensin and endothelin, and pro-inflammation and inactivation of $\mathrm{NO}$ by reactive oxygen species ${ }^{6}$. An intermittent consistency of shear stress is also a factor that can affect endothelial function. The development of plaques usually takes place in areas where shear stress is high, oscillating or retrograde, creating an inflammatory response or causing erosion and/or plaque damage ?

There is no doubt that advancing age is accompanied by loss in vessel function ${ }^{7}$. Until menopause, women generally have a lower risk of developing CVD compared to men of similar age ${ }^{8}$. Estrogen is thought to play a pivotal role in this decreased risk. Estrogen has besides antihypertensive effects, also an attenuating effect on vascular injury and atherosclerosis mediated through $\mathrm{NO}$ and angiotensin converting enzyme. Moreover, it also decreases this risk as a result of different mechanisms, in particular the beneficial effect on the lipid profile and an anti-inflammatory effect 9,10. After menopause these protective effects are lost and the risk of CVD in women is considered similar to the risk for men. Another sex difference is the clinical presentation of CVD because more women than men present with acute coronary syndrome but non-obstructive arteries. 
This implies that other mechanisms could be present besides plaque formation, such as abnormal microvascular function of the coronary arteries ${ }^{11}$. The growing body of evidence for different mechanisms underlying cardiovascular disease in women make it more relevant to also study vascular endothelial function specifically in a female population.

Our endothelium is not only interesting as a marker of cardiovascular disease. Because it lines every organ, endothelial dysfunction can be assessed in different ways and in different vascular beds. Our kidneys for example, are also lined with vascular endothelium and, even though kidney function is not considered a common parameter of endothelial function, it actually is. Damaged endothelium in the kidney results in urinary loss of albumin and a decrease in glomerular filtration rate ${ }^{12-15}$.

\section{MEASURING ENDOTHELIAL FUNCTION}

Over the years, endothelial function has been measured through different methods available. Nowadays there are several common invasive and non-invasive assessment measures. All common invasive measurements of endothelial function encompass measuring vascular response after the infusion of a vasoactive substance whilst noninvasive measurements evaluate patterns of blood flow through vessels, either with or without a temporary occlusion. During pregnancy, various methods have been used to study endothelial function. Most studies, however, have been restricted to in vitro experiments on isolated endothelial cells or vessels from maternal or foetal tissues because of the invasive nature of most of these tests. Flow mediated dilation (FMD) is a measure that, over the last decades, has increased in popularity because it can also be used as a proxy for cardiovascular health.

\section{MEASURING FLOW MEDIATED DILATION (FMD)}

FMD is a non-invasive two-dimensional ultrasound technique to measure vascular endothelial function. A damaged endothelium usually results in a decreased FMD. FMD is described as a NO endothelium-dependent process reflecting the dilation of an artery, usually of the brachial artery, when exposed to reactive hyperaemia ${ }^{16-18}$. FMD is calculated by the absolute increment in arterial diameter after deflation of a blood pressure cuff, thus inducing reactive hyperaemia, in relation to the artery diameter, given in percentages ${ }^{19}$. With FMD, endothelium reactivity can be studied in systemic arteries safely during pregnancy.

When using the brachial artery, a blood pressure cuff is placed on the forearm and a continuous measurement of blood velocity and artery diameter using duplex ultrasound is performed on the brachial artery. After several minutes of obtaining a baseline resting velocity and diameter the blood pressure cuff is inflated to $200 \mathrm{mmHg}$, and this is maintained for 5 minutes. This causes ischemia and consequently dilation of downstream 
resistance vessels via auto-regulatory mechanisms. After release of the blood pressure cuff blood flow increases inducing a brief high-flow state through the brachial artery (reactive hyperemia) to accommodate the dilated resistance vessels (see Figure 2). The resulting increase in shear stress causes the brachial artery to dilate. This reaction and the vessels recovery is recordered during several minutes. The magnitude of dilation, due to an increase in shear stress and the subsequent release of $\mathrm{NO}$, is determined by three different aspects namely: characteristics of the stimulus (amount and pattern), transduction of the vasodilator response to the surrounding smooth muscles, and structural characteristics of the vessel wall.

The exact system for detecting an increase in shear stress is thought to be related to calcium-activated potassium channels that react by shifting to an open conformation, hyperpolarizing the endothelial cell and subsequently causing calcium to enter the cell ${ }^{18,20}$ ${ }^{23}$. As such, calcium activates eNOS leading to NO synthesis and causing the surrounding smooth muscle cells to relax.
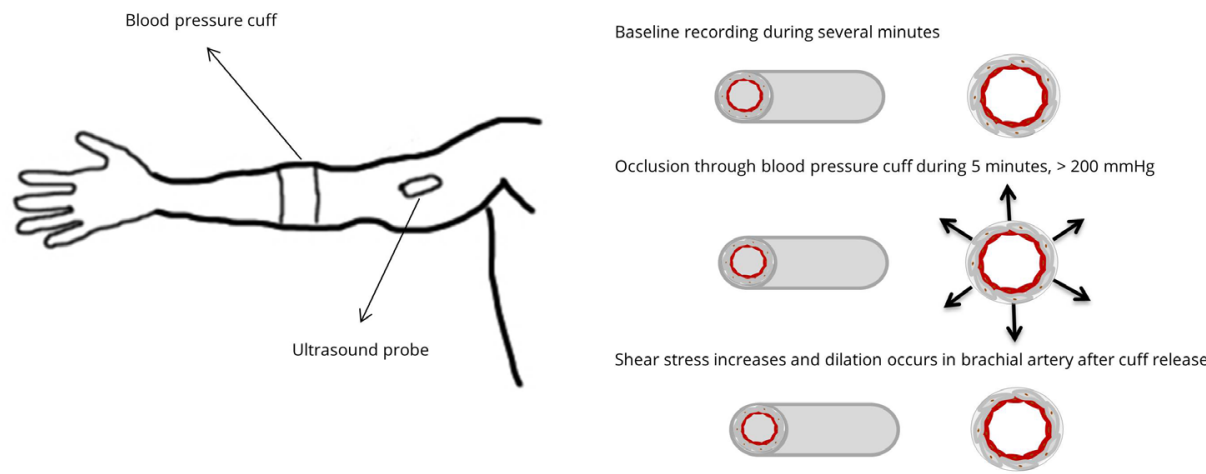

Recovery baseline recording during several minutes

Figure 2 Schematic representation of flow mediated dilation.

Since its development in 1992, FMD measurement protocols have improved substantially. Recommendations proposed in a comprehensive tutorial by Harris et el, published in 2010, represent the most recent advancements in ultrasonic measurement of FMD in an attempt to reach standardisation across research sites and to subsequently facilitate the use of FMD as a clinically relevant research too ${ }^{17}$. When measuring FMD one should keep in mind that the magnitude of the reaction can be influenced by different aspects: dietary intake (specifically caffeine, alcohol, fat and vitamin C), recent aerobic exercise, medication 
use, menstrual cycle and sympathetic stimuli. An experienced ultra-sonographer is a prerequisite to an optimal quality of the measurement. Recently it has also been suggested that FMD should be indexed for baseline diameter, through allometric scaling, considering that smaller vessels have a greater FMD response than larger vessels ${ }^{24,25}$.

\section{MEASURING KIDNEY FUNCTION}

Kidney function is generally expressed by two parameters: glomerular filtration rate (GFR), indicating the speed of filtration, and albuminuria, indicating the quality of the glomerular endothelium. GFR is determined by a multiplication of the glomerular capillary filtration coefficient and the net filtration pressure.

The capillary filtration coefficient is determined by hydraulic conductivity and the amount of surface area of glomerular capillaries. The net filtration pressure is determined by hydrostatic and oncotic pressures in the glomerulus and Bowman's capsule (see Figure 3).

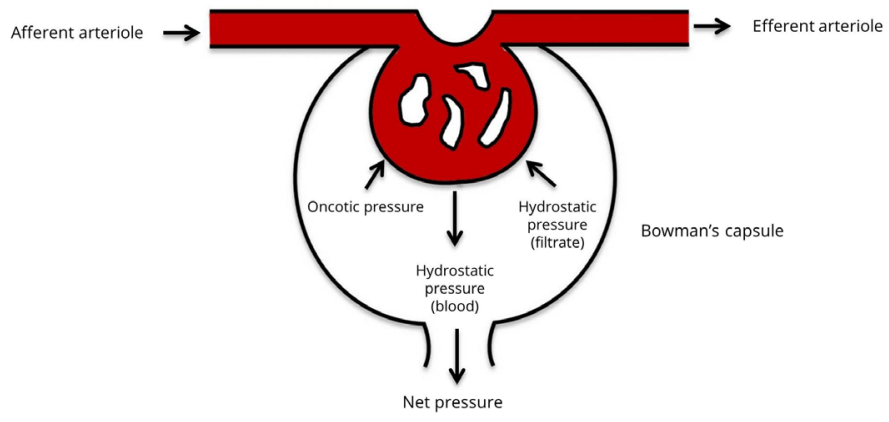

Figure 3 Schematic representation of net filtration pressure.

The filtration coefficient can be influenced by a change of the number of functional glomerular capillaries (changes in surface area) or change in the thickness of the glomerular capillary membrane (changes in hydraulic conductivity) but these are, in a healthy kidney, a stable part of the equation. Under healthy conditions, the concentration of protein in the glomerular filtrate is so low that oncotic pressure is considered zero. Net filtration pressure is therefore mostly determined by hydrostatic pressure ${ }^{26}$. 
GFR can be measured by the renal clearance of different substances. The ideal marker is a solute that is nontoxic, freely filtered at the glomerulus and neither secreted nor reabsorbed by the kidneys tubules. Over the years multiple endogenous and exogenous filtration markers have been used to evaluate kidney function ${ }^{27}$. Inulin is the most commonly used exogenous marker and considered the golden standard while creatinine is the most used endogenous marker. Measuring GFR through inulin has the disadvantages of being a time-consuming and complex procedure that requires urine catheterisation to be accurate.

Because of the disadvantages of inulin, creatinine has become widely accepted to estimate GFR even though it is less accurate. Creatinine is derived from the metabolism of creatine in skeletal muscle and from dietary meat intake and is present in a relatively constant concentration ${ }^{28}$. It is usually measured in a 24 hour urine collection in combination with serum blood creatinine, termed creatinine clearance. There are two main reasons for the inaccuracy of creatinine clearance namely, the inaccuracies in the method of collection and the fact that creatinine quantities in the blood can be influences by dietary intake and/or exercise ${ }^{29}$.

GFR estimates can be calculated by different formulas that take several factors into account, namely weight, age and ethnicity. The more commonly used formulas are Cockcroft-Gault (CG), Modification of Diet in Renal Disease (MDRD) and the more recent Chronic Kidney Disease Epidemiology Collaboration (CKD-EPI). It is relevant to note that all of these formulas are based on large cohorts of non-pregnant patients with at least mild renal insufficiency ${ }^{30-32}$. During pregnancy GFR estimates can vary in accuracy and creatinine clearance, MDRD and CG are found to underestimate GFR ${ }^{33-35}$.

Not only does GFR provide us information on kidney function, so does albuminuria. Albuminuria is the loss of albumin through the glomerular endothelium, which is indicative of the quality of the endothelium than lines the kidneys. A damaged endothelium at the glomerulus will result in (increased) loss of albumin in the urine.

\section{ENDOTHELIAL FUNCTION AND PREGNANCY}

The adaptation a female body goes through during pregnancy is extensive. Major hemodynamic changes induced by pregnancy include a reduction in systemic vascular resistance, blood volume expansion, an increase in cardiac output, sodium and water retention and a decrease in systemic blood pressure. Most adaptive changes appear early in pregnancy, reach their peak during the second trimester, and then remain relatively constant until delivery. Ultimately, they parallel optimal growth and development of the foetus. The mechanisms responsible for the pregnancy associated vasodilation and 
decrease in vascular resistance are not yet fully understood but is thought to be mostly accomplished by a decrease of endothelial vascular responsiveness to constrictors angiotensin and norepinephrine and an increased susceptibility for endothelium derived relaxing factor and NO, both vasodilators ${ }^{36}$. Estrogen and relaxin also play an important role in decreasing vascular resistance through vasodilation ${ }^{37}$.

Maternal adaptations during pregnancy result in an (temporary) increase in shear stress on the mono-cellular layer of vascular endothelium and thus triggering vascular reactivity to self-regulate vessel tone. Subsequently, local endothelial mechanisms induce vasomotor response by means of vasodilation in functionally intact endothelium as observed in conduit arteries ${ }^{20,38}$. Furthermore, besides the drop in renal resistance, these changes also induce an increased renal blood flow and subsequently an increase in GFR. When the resistance of the afferent arteriole decreases, renin is released. The release of renin causes an increase in the formation of angiotensin I, which in turn is converted to angiotensin II that constricts the kidney's efferent arterioles thereby increasing glomerular hydrostatic pressure. This results in an increase of filtration pressure and consequently an increase in GFR. Additionally to the increase in net filtration pressure, the oncotic pressure substantially decreases because of plasma volume expansion, which also contributes to the rise in GFR during pregnancy ${ }^{39}$. Interestingly, our kidneys show similar antihypertensive effects from estrogen signalling, since $\mathrm{NO}$ also participates in kidney control of extracellular fluid homeostasis ${ }^{40}$.

\section{PREECLAMPSIA AS AN ENDOTHELIAL DISEASE}

Preeclampsia is a pregnancy related syndrome that usually occurs after 20 weeks of pregnancy. Even though it has a relative small incidence of $2-8 \%$ of all pregnancies, it has a great impact on maternal and foetal morbidity and mortality. If uncontrolled it can progress to eclampsia, which is characterized by epileptic seizures. About $14 \%$ of maternal deaths worldwide are caused by preeclampsia; it is the second most occurring cause of maternal death ${ }^{41}$. There is currently no definite treatment and/or cure except for delivery.

Preeclampsia is usually a state of endothelial dysfunction that is expressed by hypertension and commonly coincided by proteinuria, disseminated intravascular coagulation, hepatic and/or kidney problems. During preeclampsia, vascular endothelial function is disturbed which can be seen by a decrease or downregulation of different factors needed for uncomplicated maternal adaptation to pregnancy. The further understanding of maladaptive responses during pregnancy could provide more insight in the pathophysiology of preeclampsia. 
What makes research concerning the aftermath of preeclampsia complicated is the difficulty to discern between a maternal predisposition for and consequence of the syndrome. There are several arguments to be made for an interaction between multiple factors. The most compelling argument is that preeclampsia is a multi-organ syndrome that may develop through different pathophysiologic pathways that vary considerably and therefore presents itself through a wide range of symptoms. Most notable predisposing maternal factors are genetics, a low plasma volume before pregnancy, obesity, age, hypertension, diabetes, renal disease, vascular disease and autoimmune disease that influence the development of preeclampsia. After pregnancy complicated by preeclampsia the endothelium seems to be affected up until a few years after delivery but there are some studies that indicate this negative effect on the endothelium might not persist over time ${ }^{42-45}$. Overall this generalized endothelial dysfunction does seem to be more prevalent after a pregnancy complicated by preeclampsia. Women with a history of preeclampsia are at a 2 - 6 fold increased risk of future cardiovascular disease and a 3 - 7 fold increased risk of future kidney disease, depending on the severity of the disease during pregnancy ${ }^{46,47}$. It seems therefore relevant to understand why this increased risk is present and what, if any, the common pathways would be. 


\section{AIMS AND OUTLINE OF THIS THESIS}

This thesis aims to provide insight in maternal adaptation to pregnancy of endothelial and kidney function, to discuss maladaptation during pregnancy and the possible long-term consequences on vascular endothelial and kidney function thereof.

CHAPTER 2 establishes reference values for flow mediated dilation (FMD) and brachial artery diameter (BAD) in pregnancy and provides insight in the physiological and pathological course of endothelial adaptation throughout human singleton pregnancies.

CHAPTER 3 systematically reviews and quantifies current literature on kidney function during pregnancy, estimates the extent of adaptation over the course of both physiological and hypertensive complicated singleton pregnancies, and determines a possible threshold to distinguishing normal from abnormal.

CHAPTER 4 discusses early systemic maternal kidney and cardiovascular adaptation to pregnancy in women with a hypertensive complicated pregnancy in their history, and defines differences in women who do and do not develop a recurrent hypertensive disorder later on in pregnancy.

CHAPTER 5 determines the prevalence of chronic kidney disease in women with a history of preeclampsia that would need monitoring of kidney function according the KDIGO guideline.

CHAPTER 6 aims to shed light on the relationship between endothelial dependent vasodilation expressed by FMD and kidney function expressed by glomerular filtration rate and microalbuminuria, as both can reflect endothelial (dys)function.

CHAPTER 7 evaluates the arterial aging process and the effects of obstetric history upon the physiologic age related decline in vascular endothelial function. 


\section{REFERENCES}

1. Esper RJ, Nordaby RA, Vilarino JO, Paragano A, Cacharron JL, Machado RA. Endothelial dysfunction: a comprehensive appraisal. Cardiovasc. Diabetol. Feb 23 2006;5:4.

2. Widlansky ME, Gokce N, KeaneyJF, Vita JA. The clinical implications of endothelial dysfunction.J. Am. Coll. Cardiol. 2003;42(7):1149-1160

3. Vane JR, Anggard EE, Botting RM. Regulatory Functions of the Vascular Endothelium. The New Eng/and Journal of Medicine. 1990;323(1):28-36.

4. Tuzcu EM, Kapadia SR, Tutar E, et al. High Prevalence of Coronary Atherosclerosis in Asymptomatic Teenagers and Young Adults. Circulation. 2001;103:2705-2710.

5. Collins P, Rosano G, Casey C, et al. Management of cardiovascular risk in the perimenopausal women: a consensus statement of European cardiologists and gynecologists. Climacteric. Dec 2007;10(6):508-526.

6. Adams MR. Clinical assessment of endothelial function. Endothelium. Nov-Dec 2006;13(6):367-374.

7. Lakatta EG. Arterial and Cardiac Aging: Major Shareholders in Cardiovascular Disease Enterprises: Part I: Aging Arteries: A "Set Up" for Vascular Disease. Circulation. 2003;107(1):139-146.

8. Mozaffarian D, Benjamin EJ, Go AS, et al. Heart Disease and Stroke Statistics-2016 Update. A Report From the American Heart Association. 2016;133(4):e38-e360.

9. Robert H. Knopp, Pathmaja Paramsothy, Barbara M. Retzlaff, et al. Sex Differences in Lipoprotein Metabolism and Dietary Response: Basis in Hormonal Differences and Implications for Cardiovascular Disease. Current Cardiology Reports 2006;8:452-459.

10. Gilliver SC. Sex steroids as inflammatory regulators.J. Steroid Biochem. Mol. Biol. May 31 2010;120(2-3):105-115.

11. Murthy VL, Naya M, Taqueti VR, et al. Effects of sex on coronary microvascular dysfunction and cardiac outcomes. Circulation. Jun 17 2014;129(24):2518-2527.

12. Malyszko J. Mechanism of endothelial dysfunction in chronic kidney disease. Clin. Chim. Acta. Oct 09 2010;411(19-20):1412-1420.

13. Friden $\mathrm{V}$, Oveland $\mathrm{E}$, Tenstad $\mathrm{O}$, et al. The glomerular endothelial cell coat is essential for glomerular filtration. Kidney Int. Jun 2011;79(12):1322-1330

14. Ballermann BJ. Contribution of the endothelium to the glomerular permselectivity barrier in health and disease. Nephron Physiol. 2007;106(2):p19-25.

15. Salmon AH, Satchell SC. Endothelial glycocalyx dysfunction in disease: albuminuria and increased microvascular permeability. J. Pathol. Mar 2012;226(4):562-574.

16. Corretti MC, Anderson TJ, Benjamin EJ, et al. Guidelines for the ultrasound assessment of endothelialdependent flow-mediated vasodilation of the brachial artery. J. Am. Coll. Cardiol. 2002;39(2):257-265.

17. Harris RA, Nishiyama SK, Wray DW, Richardson RS. Ultrasound assessment of flow-mediated dilation. Hypertension. May 2010;55(5):1075-1085.

18. Miura H, Wachtel RE, Liu Y, et al. Flow-Induced Dilation of Human Coronary Arterioles Important Role of Ca2 -Activated K Channels Circulation. 2001;103:1992-1998.

19. Uehata A, Lieberman EH, Gerhard MD, et al. Noninvasive assessment of endothelium-dependent flowmediated dilation of the brachial artery. Vasc. Med. 1997;2(2):87-92

20. Pohl U, Holtz J, Busseand R, Bassenge E. Crucial Role of Endothelium in the Vasodilator Response to Increased Flow in Vivo. Hypertension. 1986;8:37-44.

21. Olesen SP, Clapham DE, Davies PF. Haemodynamic shear stress activates a K+ current in vascular endothelial cells. Nature. Jan 14 1988;331(6152):168-170.

22. Cooke JP, Jr. ER, Andon NA, Loscalzo J, Dzau VJ. Flow Activates an Endothelial Potassium Channel to Release an Endogenous Nitrovasodilator. J. Clin. Invest. 1991;88:1663-1671. 
23. Joannides R, Haefeli WE, Linder L, et al. Nitric-Oxide Is Responsible for Flow-Dependent Dilatation of Human Peripheral Conduit Arteries in-Vivo. Circulation. Mar 1 1995;91(5):1314-1319.

24. Atkinson G, Batterham AM. The percentage flow-mediated dilation index: a large-sample investigation of its appropriateness, potential for bias and causal nexus in vascular medicine. Vasc. Med. Dec 2013;18(6):354-365.

25. Atkinson G, Batterham AM, Thijssen DH, Green DJ. A new approach to improve the specificity of flow-mediated dilation for indicating endothelial function in cardiovascular research. J. Hypertens. Feb 2013;31(2):287-291.

26. Guyton AC, Hall JE, Lhmeier TE, Jackson TE, Manning RD, Jr. The Ninth J. A. F. Stevenson Memorial Lecture: The many roles of the kidney in arterial pressure control and hypertension. Can. J. Physiol. Pharmacol. Jun 1981;59(6):513-519.

27. Lamb EJ, Stevens PE. Estimating and measuring glomerular filtration rate: methods of measurement and markers for estimation. Curr. Opin. Nephrol. Hypertens. May 2014;23(3):258-266.

28. Stevens LA, Levey AS. Measurement of kidney function. Med. Clin. North Am. May 2005;89(3):457-473.

29. Stevens LA, Coresh J, Greene T, Levey AS. Assessing kidney function--measured and estimated glomerular filtration rate. N. Engl. J. Med. Jun 8 2006;354(23):2473-2483.

30. Levey AS, Stevens LA, Schmid CH, et al. A new equation to estimate glomerular filtration rate. Ann. Intern. Med. May 05 2009;150(9):604-612.

31. Levey AS, Bosch JP, Lewis JB, Greene T, Rogers N, Roth D. A more accurate method to estimate glomerular filtration rate from serum creatinine: a new prediction equation. Modification of Diet in Renal Disease Study Group. Ann. Intern. Med. Mar 16 1999;130(6):461-470.

32. Cockcroft DW, Gault MH. Prediction of creatinine clearance from serum creatinine. Nephron. 1976;16(1):31-41.

33. Koetje PM, Spaan J, Kooman JP, Spaanderman MEA, Peeters LLH. Pregnancy reduces the accuracy of the estimated glomerular filtration rate based on Cockroft-Gault and MDRD formulas. Reprod. Sci. May 2011;18(5):456-462.

34. Ahmed SB, Bentley-Lewis R, Hollenberg NK, Graves SW, Seely EW. A comparison of prediction equations for estimating glomerular filtration rate in pregnancy. Hypertens. Pregnancy. 2009;28(3):243-255.

35. Smith MC, Moran P, Ward MK, Davison JM. Assessment of glomerular filtration rate during pregnancy using the MDRD formula. Br. J. Obstet. Gynaecol. Jan 2008;115(1):109-112.

36. Anders Per Faber-Swenson SPMOC, Walters WAW. Endothelial cell function enhancement in a late normal human pregnancy. Aust. N. Z. J. Obstet. Gynaecol. 2004(44):525-529.

37. Chakrabarti S, Morton JS, Davidge ST. Mechanisms of estrogen effects on the endothelium: an overview. Can. J. Cardiol. Jul 2014;30(7):705-712.

38. Lie M, Sejersted OM, Kiil F. Local Regulation of Vascular Cross Section during Changes in Femoral Arterial Blood Flow in Dogs. CircaUuon Rtitmcb. 1970;27:727-737.

39. Cheung KL, Lafayette RA. Renal physiology of pregnancy. Adv. Chronic Kidney Dis. May 2013;20(3):209-214.

40. McGuire BB, Watson RW, Perez-Barriocanal F, FitzpatrickJM, Docherty NG. Gender differences in the reninangiotensin and nitric oxide systems: relevance in the normal and diseased kidney. Kidney Blood Press. Res. 2007;30(2):67-80.

41. Say L, Chou D, Gemmill A, et al. Global causes of maternal death: a WHO systematic analysis. Lancet Global Health. Jun 2014;2(6):E323-E333.

42. Hamad R, Eriksson MJ, A S, Hamsten A, Bremmea K. Decreased flow-mediated dilation is present 1 year after a pre-eclamptic pregnancy. J. Hypertens. 2007;25(2301-2307).

43. Weissgerber TL, Milic NM, Milin-Lazovic JS, Garovic VD. Impaired Flow-Mediated Dilation Before, During, and After Preeclampsia: A Systematic Review and Meta-Analysis. Hypertension. Feb 2016;67(2):415-423.

44. Lampinena KH, Ronnbackb M, Kaajaa R, Groop P-H. Impaired vascular dilatation in women with a history of pre-eclampsia. J. Hypertens. 2006;24:751-756. 
45. Sandvik MK, Leirgul E, Nygard O, et al. Preeclampsia in healthy women and endothelial dysfunction 10 years later. Am. J. Obstet. Gynecol. Dec 2013;209(6):569 e561-569 e510.

46. Bjørn Egil Vikse, Lorentz M. Irgens, Torbjørn Leivestad, Rolv Skjærven, Iversen BM. Preeclampsia and the Risk of End-Stage Renal Disease. N. Engl. J. Med. 2008;359:800-809.

47. McDonald SD, Malinowski A, Zhou Q, Yusuf S, Devereaux PJ. Cardiovascular sequelae of preeclampsia/ eclampsia: a systematic review and meta-analyses. Am. Heart J. Nov 2008;156(5):918-930. 

d

4

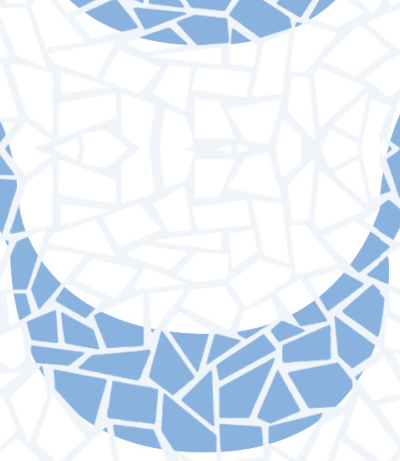

40.2
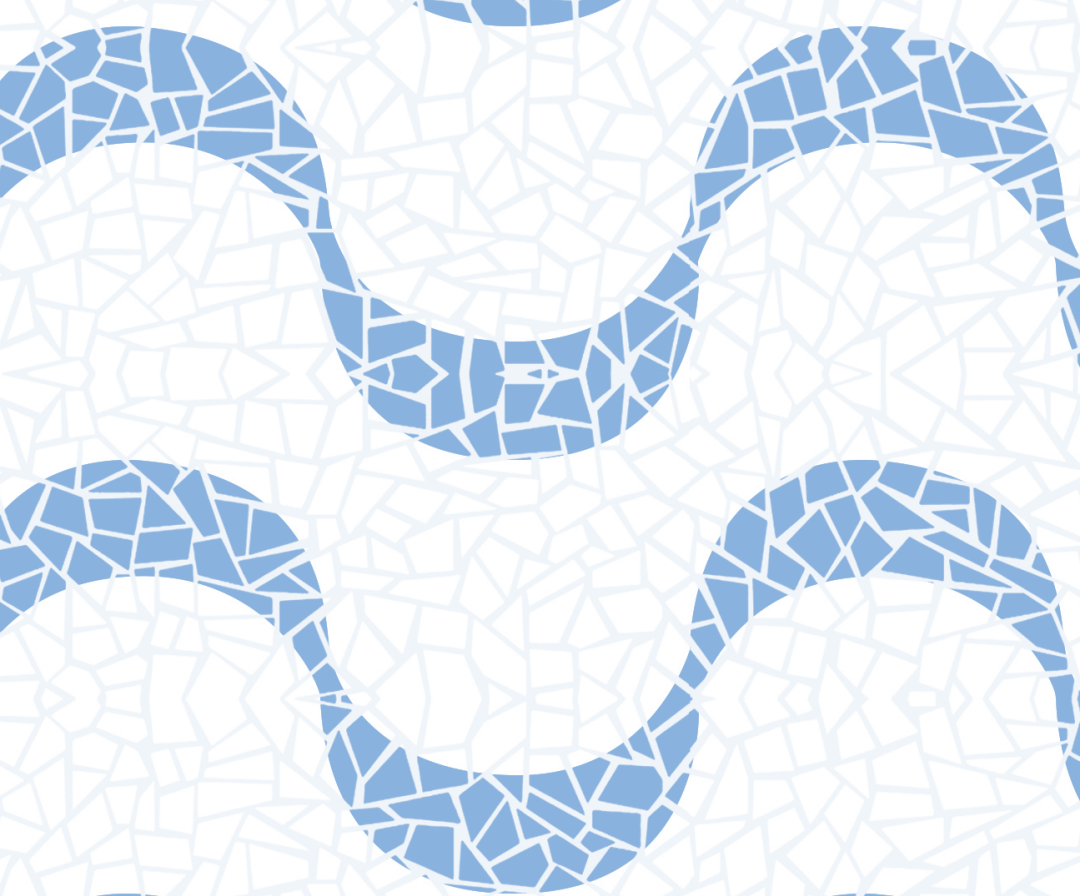

$4 \leq 1$

पर्थ

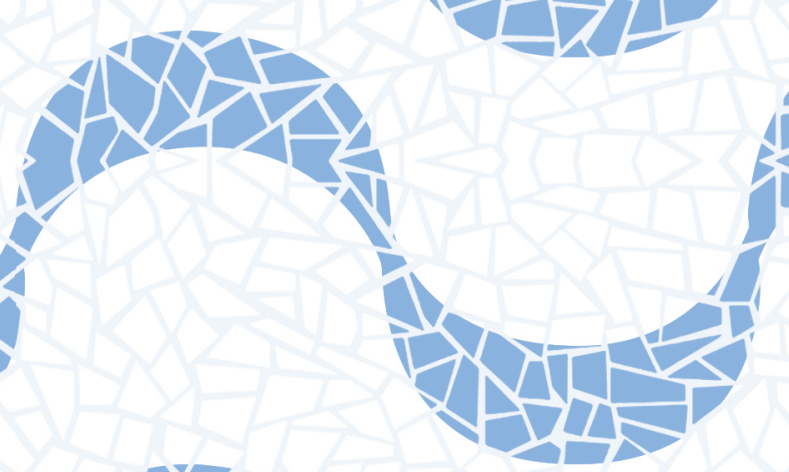

करो
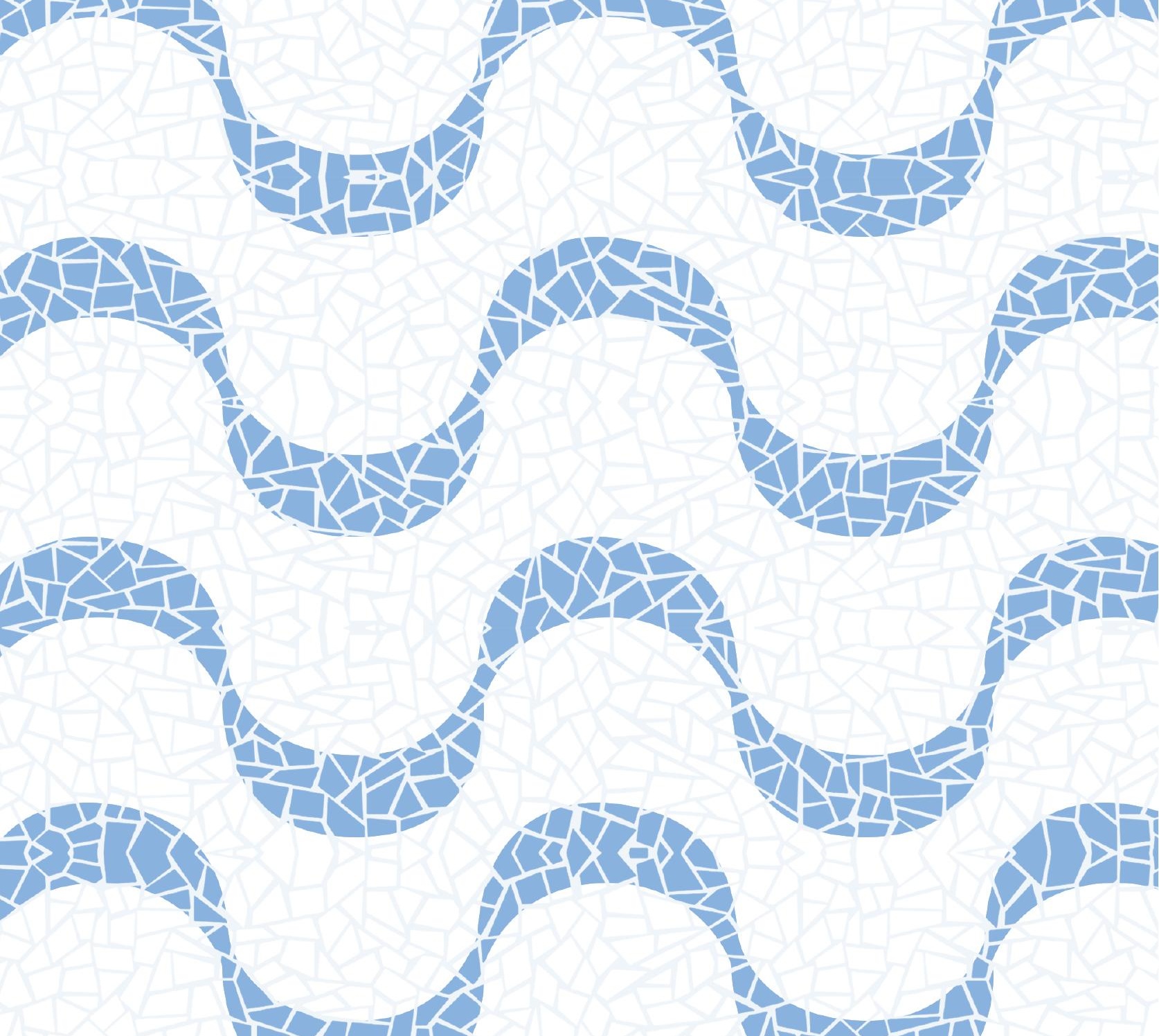


\title{
CHAPTER 2
}

\section{Physiologic adaptation of endothelial function to pregnancy: a systematic review and meta-analysis}

\author{
Veronica A. Lopes van Balen \\ Tessa A.G. van Gansewinkel \\ Sander de Haas \\ Sander M.J. van Kuijk \\ Joris van Drongelen \\ Chahinda Ghossein-Doha \\ Marc E.A. Spaanderman
}

Ultrasound Obstetrics and Gynecology; 2017 December 50(6):697-708 


\section{ABSTRACT}

INTRODUCTION The purpose of this systematic review and meta-analysis is to establish reference values for flow mediated dilatation (FMD) and brachial artery diameter (BAD) in pregnancy and to provide insight in the physiological and pathological course of endothelial adaptation throughout human singleton pregnancies.

METHODS A meta-analysis was performed following a systematic review of current literature on FMD, as a derivative for endothelial function and BAD, throughout uncomplicated and complicated pregnancies. PubMed (NCBI) and Embase (Ovid) electronic databases were used for this literature search which was performed from inception to June $9^{\text {th }}$ 2016. To allow judgment of changes as compared to a nonpregnant state, studies were required to report both a non-pregnant mean reference of FMD (matched control group, prepregnancy or postpartum measurement) and mean FMD during a predetermined and reported gestational age. Pooled mean differences between the reference and pregnant FMD values were calculated.

RESULTS Fourteen studies which enrolled 1231 participants met the selection criteria. Publication dates ranged from 1999 to 2014. In uncomplicated pregnancies FMD was increased in the second and third trimester. Between 15 and 21 weeks of gestation absolute FMD increases the most by a mean $(95 \% \mathrm{Cl})$ of $1.89 \%(0.2-3.5 \%)$. This was a relative increase of $22.5 \%(3.0 \%-42.0 \%)$ compared to non-pregnant reference. BAD increased progressively in a steady manner but did not reach significance in the first half of the second trimester. We could not discern differences in FMD and BAD between complicated and uncomplicated pregnancies at 29 - 35 weeks' gestation, reported in three studies that met our inclusion criteria. Despite the increase in FMD and BAD throughout gestation both reference curves were characterized by wide $95 \%$ confidence intervals.

CONCLUSION During healthy pregnancy both endothelium-dependent vasodilatation and BAD increase. Women with a complicated pregnancy had FMD values within the lower range when compared to uncomplicated pregnancies but, as a group, did not differ from each other. 


\section{INTRODUCTION}

A physiological pregnancy is characterized by substantial hemodynamic adaptations of the maternal cardiovascular system. Major hemodynamic changes include an increase in cardiac output, sodium and water retention leading to plasma volume expansion, and reductions in systemic vascular resistance and systemic blood pressure ${ }^{1-10}$. To accommodate the increased arterial flow, the arterial system has to adapt to keep endothelial shear rates within healthy boundaries. The mechanisms responsible for the pregnancy associated vasodilation are not yet fully understood but it is thought to be mostly accomplished by a decrease of endothelial vascular responsiveness to constrictors angiotensin II and norepinephrine and an increased availability and susceptibility for endothelium- derived relaxing factor and nitric oxide (NO). Moreover oestrogen and progesterone, which increase steadily during pregnancy are also known to stimulate NO production and may account, at least partly, for the observed vascular functional adjustment ${ }^{11-14}$. Several studies showed enhanced endothelial function during normal pregnancy reflected by an increased flow mediated dilation (FMD) and brachial artery diameter (BAD) ${ }^{15,16 .}$

Gestational hypertensive disorders (GHD) and foetal growth restriction (FGR), as part of the placenta syndrome, are believed to be elicited by a pathological placental adaptation and/ or maternal vascular vulnerability, characterized by endothelial dysfunction. The increased vascular resistance seen during GHD and FGR relates to a decreased NO production or diminished response to NO in the vascular smooth muscle cells indicating an underlying endothelial problem ${ }^{17}$. FMD has been widely used to determine endothelial function ${ }^{18}$. It can be used as a non-invasive two-dimensional ultrasound technique of the brachial artery to measure vascular endothelial function. Several studies demonstrated endothelial dysfunction during GHD and FGR, also shown by a decreased FMD, when compared to uncomplicated pregnancy ${ }^{19,20}$. However, endothelial adaptation to pregnancy and thereafter is uncertain. Moreover, the physiological pattern is unclear due to contradictory study results $16,21,22$. We hypothesised that endothelium dependent FMD gradually increases throughout gestation. This meta-analysis aimed to systematically review and quantify current literature on endothelial adaptation during pregnancy, with FMD as the derivative, to estimate the extent of adaptation over the course of both uncomplicated and complicated pregnancies. We aim to construct, based on data from the included studies, reference curves for brachial FMD and BAD. 


\section{METHODS}

FMD is described as a NO- and endothelium-dependent process reflecting the dilatation of an artery when exposed to reactive hyperaemia 18,23,24. The percentage flow mediated dilatation (FMD\%) is estimated as the absolute increment in brachial arterial diameter after deflation of a blood pressure cuff, thus inducing hyperaemia, in comparison to the $B A D{ }^{25}$. A decreased FMD response reflects endothelial dysfunction and is therefore an indication of endothelial health ${ }^{26,27}$.

\section{LITERATURE SEARCH}

Studies evaluating endothelial function during uncomplicated and complicated pregnancies were searched using PubMed (NCBI) and Embase (Ovid). These electronic databases provided publications from 1952 and 1972, respectively, up until June $9^{\text {th }}$ 2016. The search strategy focused on pregnancy, both uncomplicated and complicated, and FMD as detailed in Table 1. The complicated pregnancy component was subdivided in pregnancy-induced hypertension, FGR, preeclampsia and gestational diabetes. All components consist of free search terms in titles and abstracts and MeSH and Emtree terms for, respectively, Pubmed and Embase databases. Limits were set on humans and journal articles. Reference lists of all included studies were reviewed for additional studies.

\section{SELECTION OF STUDIES}

Studies were qualified for inclusion following a two-phased selection process. All articles obtained from the search were screened independently for eligibility by two investigators $(V L V B, T V G)$ based on title and abstract only. Articles were eligible if the method of brachial FMD measurements, as a proxy for endothelial function, was cited. Discrepancies were resolved by mutual agreement. Subsequently, articles that passed the first selection phase were read completely and screened for inclusion based on the inclusion and exclusion criteria by the same investigators. Studies were eligible for inclusion if they reported original data, including: mean FMD (\%) during human singleton pregnancies with either standard deviation (SD), standard error (SE), or $95 \%$ confidence interval $(95 \% \mathrm{Cl})$. We assumed that FMD values were normally distributed. In addition, studies had to include a reference FMD measurement of either non-pregnant, pre-pregnant or ( $\geq 6$ weeks) post-partum controls. Pre-pregnant and postpartum measurements subjects were also measured during pregnancy. This was relevant to be able to calculate differences between pregnant and non-pregnant FMD values within studies. 
Studies that did not measure FMD on the brachial artery were excluded. No restrictions were set on age, weight, height, ethnicity or parity. Additionally, studies assessing women with a pre-existing cardiovascular history or reporting data in other languages than English, Dutch, Spanish, Italian, Portuguese or German were excluded.

Studies were not excluded based on the use of medication, but were excluded based on participants' smoking status. Furthermore, intervention studies, case reports and reviews were also excluded. In case of duplicate reporting of experimental data the most recent publication was used.

\section{DATA EXTRACTION}

Data regarding study characteristics, anthropometric measures, effect measures and method used to measure brachial FMD were extracted from the selected studies. With reference to study characteristics the following was obtained: authors, year of publication, study design, sample size, population description, inclusion and exclusion criteria and key conclusions. Additionally, extracted subject characteristics consist of age, non-pregnant weight and body mass index (BMI), height, parity, gravidity, body surface area, heart rate (HR), systolic blood pressure (SBP), diastolic blood pressure (DBP), mean arterial pressure (MAP), gestational age at time of FMD measurement, birth weight of the offspring, and gestational age at delivery. For women with a complicated pregnancy gestational age at onset of the hypertensive disorder was also documented. Effect measures include FMD as primary outcome and BAD as a secondary outcome. Furthermore, data relating to ultrasound assessment of endothelial function were extracted as described in a tutorial by Harris et el. ${ }^{23}$. Included in this tutorial were the essential elements of FMD measurements, including appropriate ultrasound technology (high-resolution, multi-frequency linear ultrasound Doppler probe, duplex mode, angle steer and insonation angle correction, ECG gating, intensity-weighted velocity calculations), subject preparation (vitamin supplementation, medications, tobacco use, caffeine, previous exercise/rested state, fasted state, adequate acclimatization, repeated measurements), baseline measurements (baseline arterial diameter and baseline blood velocity), vascular occlusion (the cuff type, cuff position, cuff duration), reactive hyperaemia measurements (temporal kinetics of arterial diameters and blood velocities and calculation of shear rate) and FMD analysis (edge detection software, calculation of FMD, normalization of FMD). Each included study was given a percentage of optimality, based on the sum of optimal items. This data was used to evaluate the quality of FMD measurement. All essential elements were of equal importance and weighed accordingly in the total score. 
Studies with a positive score $\geq 60 \%$ were defined as high quality (HQ), those scoring $\geq 30 \%$ and $<60 \%$ as moderate quality (MQ) and those scoring $<30 \%$ as low quality (LQ). Requests were made to retrieve additional information from the authors of the included articles if data were unclear or incomplete ${ }^{20}$.

\section{QUALITY ASSESSMENT}

The quality and risk of bias of included studies were assessed independently by two investigators (VLVB , TVG) according to a modified set of items as reported in the Quality in Prognosis Study (QUIPS) tool ${ }^{28}$. This modification was made to suit the purposes of this review. A plus or minus or interrogation mark (that counts as a minus and was used when item was not applicable) was allocated per study for risk of bias on five domains: study participation, study attrition, variable measurement, data reporting, and study design. All domains were deemed of equal importance, and thus weighed accordingly in the total score. In case of loss to follow-up, studies were solely scored for study attrition. Studies with a positive score $\geq 60 \%$ were defined as high quality (HQ), those scoring $\geq 30 \%$ and $<60 \%$ as moderate quality (MQ) and those scoring < $30 \%$ as low quality (LQ).

\section{DATA AND STATISTICAL ANALYSIS}

FMD measurements were categorized in five different intervals of gestational age $(<14$ 15-21, 22-28, 29-35, and 36-41 weeks). These gestational age intervals were adapted from Abudu et el. ${ }^{29}$ and enabled a most precise categorization of almost all available FMD measurements. FMD and diameter change was calculated separately for these predefined intervals using a random-effects model as described by DerSimonian and Laird ${ }^{30}$. SD was obtained from SE or 95\% Cl and calculated for combined groups according to the Cochrane handbook for systematic review of interventions whenever necessary ${ }^{31}$. The random-effects model allows for inter-study variation and was chosen since observational data of different pregnant populations were used. Egger's regression test for funnel plot asymmetry was performed to test for the presence of publication bias ${ }^{32}$.

The primary outcome was the mean difference in FMD between pregnancy and the reference value, reported with a $95 \% \mathrm{Cl}$. In case of a study reporting both non-pregnant controls and post-partum reference measurements, the latter was selected to reduce measurement errors. When a study provided multiple post-partum values, the last one was obtained as a reference measurement. We reported the absolute FMD increase from reference in percentage and the relative increase from reference, also in percentages. Some longitudinal studies reported reference and pregnant FMD of the same women 16,20,33,34. Point estimates are unaffected by these dependent observations. 
If the authors did not present sufficient information to determine the covariance between measurements, instead of estimating (a range of) covariance, we ignored their dependence. Since the variance of the mean difference decreases as the covariance between the two measurements increases, not taking the covariance into account can only have resulted in slightly conservative estimates of precision.

The ratio between total heterogeneity and total variability (I-squared statistic $\left.\left(I^{2}\right)\right)$ was computed as a measure of heterogeneity. $I^{2}$ can distinguish true heterogeneity from sampling variance and is expressed as a percentage ${ }^{35}$. Sources of heterogeneity and differences between uncomplicated and complicated pregnancies were investigated by meta-regression analyses using a mixed-effects model. We considered type of reference, FMD measurement quality and study quality as sources of heterogeneity. All variables were corrected for gestational age. The meta-analyses and meta-regression analyses were performed in R version 3.2.3 using the meta package ${ }^{36,37}$. 


\section{RESULTS}

\section{STUDY AND DATA SELECTION}

The literature search yielded 2424 articles, after duplicate removal, from both Embase and Pubmed electronic databases (Figure 1). After screening based on title and abstract we identified 181 studies eligible for full-article analysis. Following exclusion, largely due to the lack of FMD reference measurements $(n=71), 14$ articles were ultimately selected. Studies excluded based on study design did either: not measure FMD, solely reported correlations or only stated non-pregnant and postpartum brachial FMD measurements. Additionally, one article was excluded due to suspicion of duplicate reporting ${ }^{38}$, while five other articles were excluded because of unobtainable data from abstracts ${ }^{39-43}$. One article was excluded based on FMD measurement method, due to measurements of the radial artery rather than the brachial artery ${ }^{44}$. We did not find additional studies after screening reference lists. For all included studies reporting more than one dataset, only data with an eligible reference value ${ }^{45}$, FMD measurement value, ${ }^{20}$ and cross-sectional data ${ }^{16}$ were included. Two studies included both non-pregnant controls and postpartum controls ${ }^{15,34}$. One study reported FMD data in healthy children and adults of both sex with and without a history of stroke or coronary heart disease and healthy pregnant women. Only the FMD values of healthy pregnant women were included along with the reference FMD ${ }^{45}$. In another study FMD data were presented in active pregnant, sedentary pregnant, active non-pregnant and sedentary non-pregnant women, but solely the combined data of pregnant and non-pregnant were included ${ }^{21}$. In a study consisting of non-smokers and smokers, only non-smoker FMD data were included because of the known negative effect of smoking on endothelial function ${ }^{46}$. The 14 articles selected for analysis included 387 non-pregnant, 542 healthy pregnant, 223 postpartum, and 79 GHD FMD measurments.

\section{CHARACTERISTICS}

Study characteristics and anthropometric measures of study participants included in this review and meta-analyses are described in Tables 2 and 3, for uncomplicated pregnancies and pregnancies complicated by GHD, respectively. For most studies overall characteristics are presented, since these did not report subgroup characteristics. Pregnancies complicated by preeclampsia 47,48 and gestational hypertension ${ }^{20}$ were indexed as pregnancies complicated by GHD.

\section{DATA EXTRACTION}

Participant's anthropometric data were insufficiently and infrequently reported by the authors. It was therefore impossible to analyse the contribution of these variables to the heterogeneity observed. 

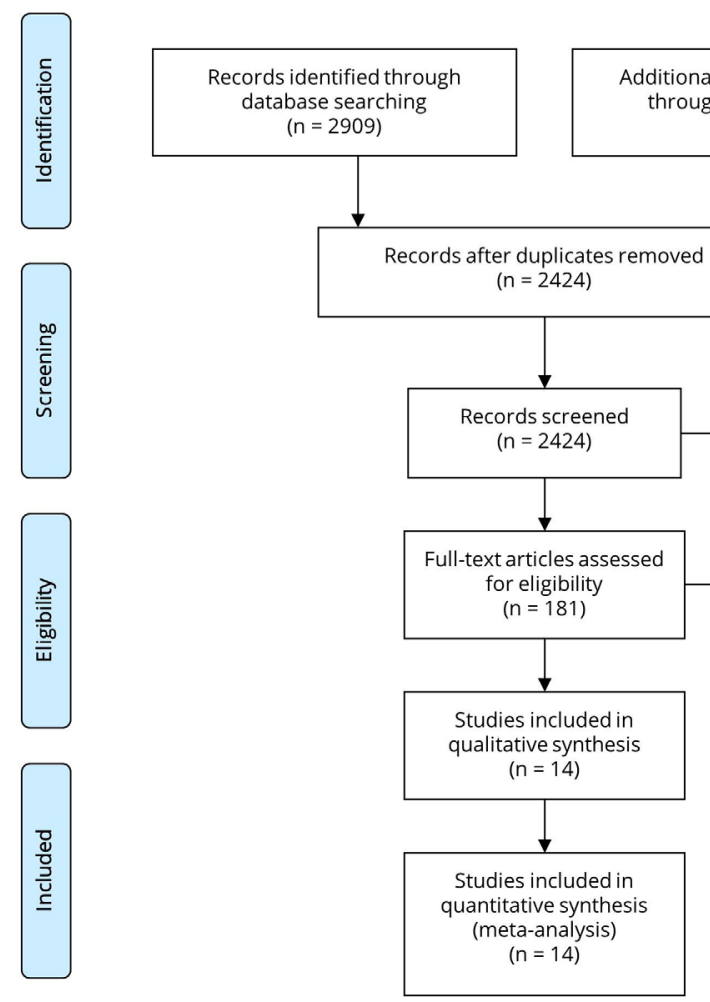

Aditional records identified

rough other sources $(n=0)$

Figure 1 Flow chart for selection, inclusion and exclusion.

\section{QUALITY ASSESSMENT}

Quality assessment of included studies was subdivided in HQ ( $n=2), M Q(n=10)$ and LQ $(n=2)$ as illustrated in Table 4. Outcome scores varied between 24\% and 65\% (median 47\% IQR 37 - 53\%). All studies fulfilled two items; 'Clear reporting of gestational age' and an 'equal method and setting for all study participants throughout follow-up'. All but three stated a mean of the time frame of measurements at gestational age $45,48,49$. However these data were later provided by the author of one article ${ }^{49}$. Items that scored lowest were a pre-pregnant FMD value as reference $(n=0)$ and adequate description of ethnicity $(n=0)$.

FMD quality assessment of included studies was subdivided in MQ $(n=4)$ and LQ $(n=$ 10) studies, with no HQ studies (Table 5). Outcome scores varied between $4 \%$ and $42 \%$ (median 23\% IQR 17-32\%). These scores are predominantly the result of insufficient and/ or absence of data reporting method, rather than elements not being optimal. 
The meta-regression analysis, to interpret heterogeneity, in terms of study-level covariates, showed a significant statistical association between FMD course and different study qualities respectively (LQ vs. MQ vs. HQ $(0.06 \leq \mathrm{p} \leq 0.02))$. There was no significant effect of gestational age on the course of FMD measurement ( $p=0.07$ ). The FMD measurement quality was not significant ( $p=0.38$ ), nor the type of reference measurement (postpartum vs. non-pregnant controls, $p=0.92$ ). The meta-regression analysis for BAD showed a significant effect of gestational age $(p=0.02)$, indicating a considerable increase during the course of pregnancy. There was no effect on the BAD course and different study qualities (LQ vs. MQ vs. HQ, $0.15 \leq \mathrm{p} \leq 0.19$ ), nor the type of reference measurement (postpartum vs. non-pregnant controls, $p=0.66$ ) or FMD measurement quality $(p=0.09)$.

\section{FLOW MEDIATED DILATATION}

Between 15 and 21 weeks of gestation, FMD showed the greatest increases of $1.89 \%$ (0.2 - 3.5\%). This was a relative increase of 22.5\% (3.0\% - 42.0\%) compared to reference. In addition, the highest increase in FMD disclosed in this review was 7.27\% (4.2 - 10.3\%), which was substantially larger compared to the other studies in this category ${ }^{33}$. Seeing that the second trimester in this review reported its greatest increase, and more importantly, a specific study ${ }^{33}$ showed a substantially larger effect than the other articles, this raised a concern regarding its influence on the mean percentage increase during this interval. Thus, a sub analysis of the forest plots was performed without this particular study, that still resulted in a significant increase of 1.05\% (0.09-2.02\%) during the 15-21 weeks interval. One study reported FMD data between 14 and 16 weeks' gestation and was allocated to this interval in the analysis ${ }^{45}$. Forest plots of FMD course throughout uncomplicated pregnancies compared to reference values are depicted in Figure 2. Funnel plot asymmetry was not statistically significant at any interval $(0.22<p<0.62)$. FMD was increased to a lesser degree with $0.94 \%$ (0.0 - 1.9\%), relative $10.4 \%(0.0-20.7 \%)$, in the last half of the second trimester at 22 - 28 weeks' gestation. At 29 - 35 weeks of pregnancy FMD was increased by $1.24 \%$ (0.3 - 2.2\%), relative 13.9\% (3.3 - 24.6\%), which is greater than the previous interval, but lesser than the first half of the second trimester. Most studies reported data on this interval. The greatest increase in FMD reported in this category was 5.40\% (2.0 - 8.7\%). Lastly, no increase was seen between 36 and 41 weeks' gestation compared to reference conditions. One study reported FMD data between 32 and 39 weeks' gestation and was allocated to the last interval in the analysis ${ }^{45}$.

A forest plot of the FMD course during pregnancies complicated by GHD compared to reference values is illustrated in Figure 3. Included studies report data on FMD during complicated pregnancies between 29 and 35 weeks. There was no significant increase in FMD, 1.2\% (-0.5 - 2.9\%) relative 13.9\% (-5.9\% - 33.7\%), between 29 and 35 weeks compared to reference values, nor was this different from uncomplicated pregnancies $(p=0.77)$. 


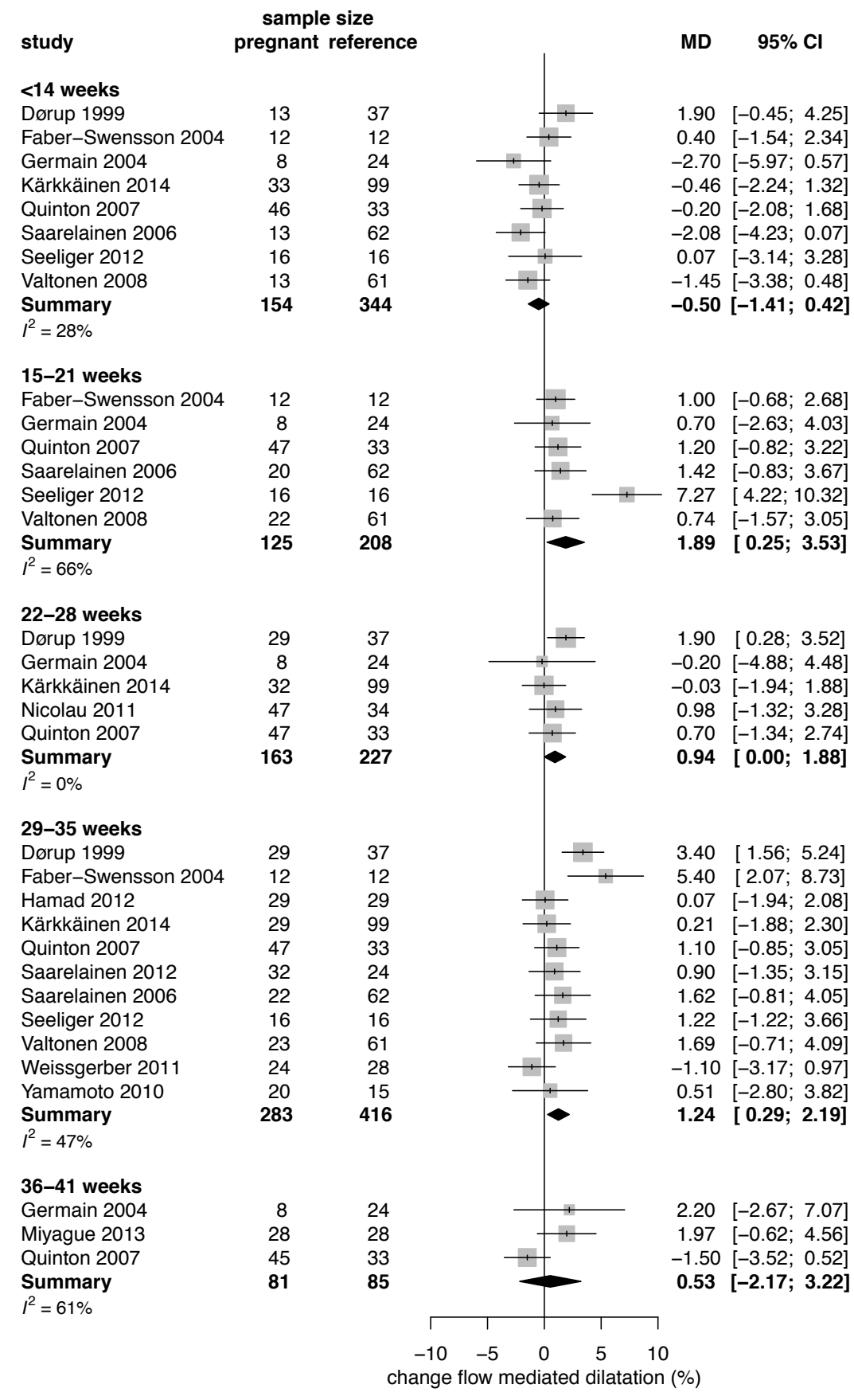

Figure 2 Forest plot of flow mediated dilatation (FMD) during physiologic pregnancies at $<14$ weeks, 15 - 21 weeks, 22 - 28 weeks, 29 - 35 weeks, and 36 - 41 weeks' gestation compared to the non-pregnant reference value. FMD is reported as mean difference (MD) between pregnant and reference $\mathrm{FMD}$, with $95 \% \mathrm{Cl}$, as a percentage. 
Study

29-35 weeks

Hamad 2012

Saarelainen 2012

Yamamoto 2010

Summary

$I^{2}=25 \%$
Sample size

Pregnant Reference

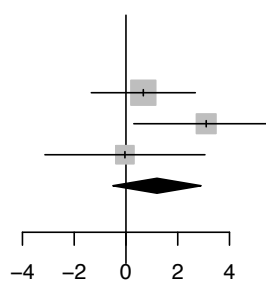

MD

$95 \% \mathrm{Cl}$

$0.67 \quad[-1.34 ; 2.68]$

$3.10 \quad[0.30 ; 5.90]$

$-0.04[-3.13 ; 3.05]$

$1.20[-0.51 ; 2.90]$

Change flow mediated dilatation (\%)

Figure 3 Forest plot of flow mediated dilatation (FMD) during pregnancies complicated with preeclampsia and pregnancy-induced hypertension at 29 - 35 weeks' gestation compared to the reference value. FMD is reported as mean difference (MD) between pregnant and reference FMD, with $95 \% \mathrm{Cl}$, as a percentage.

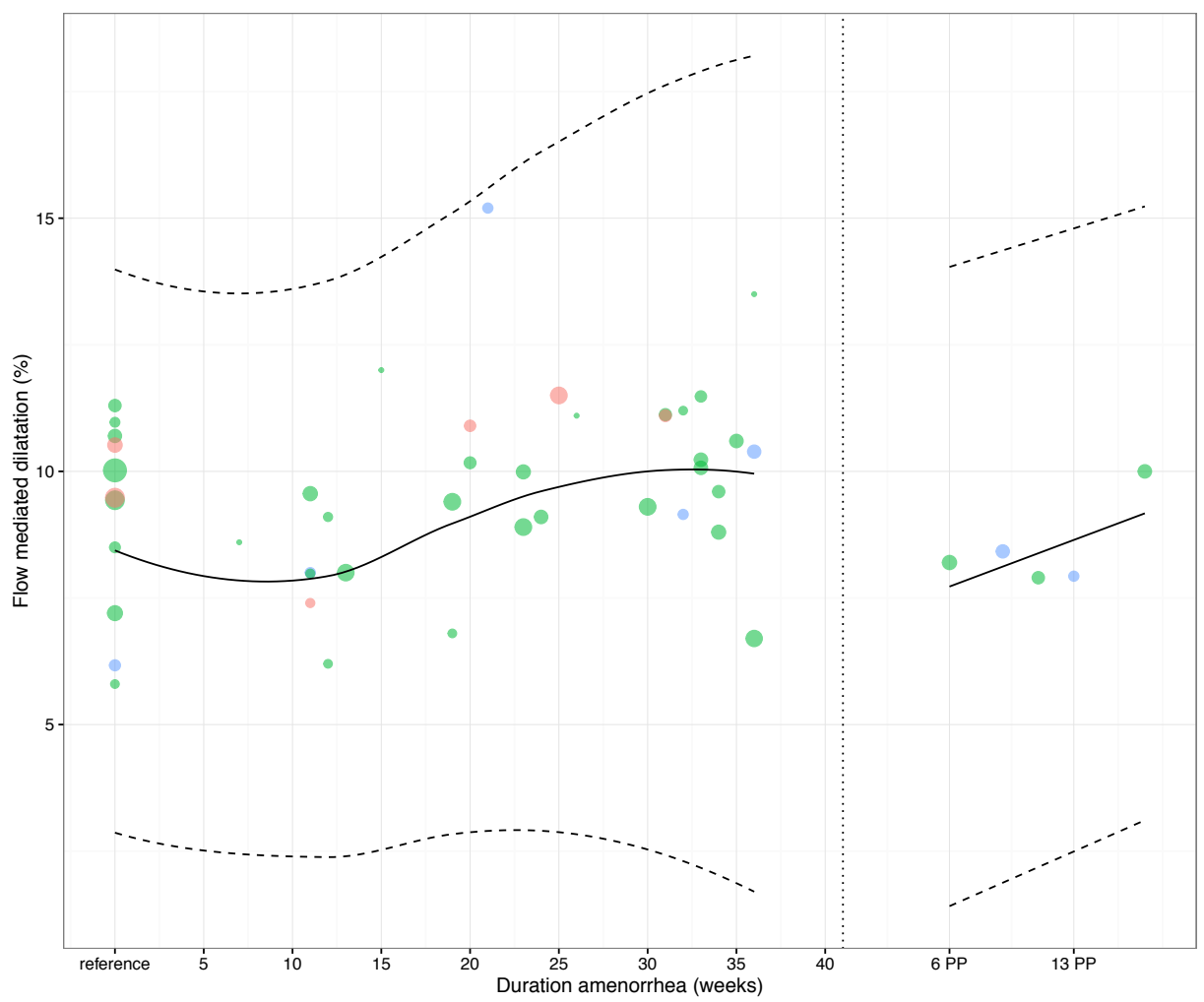

Figure 4 Flow mediated dilatation as a percentage during physiologic pregnancies and postpartum (PP) in percentage. The circle size indicates the sample size of the point estimate. The colour of the circle refers to the quality assigned to the study: red $=L Q$, green $=M Q$, blue $=H Q$. Curve fit is weighted by the inverse variance and plotted with $5^{\text {th }}$ and $95^{\text {th }}$ percentiles. The $50^{\text {th }}$ percentile is represented by a solid line. 
In order to construct reference values for FMD throughout physiological, uncomplicated, pregnancy the mean FMD values of both healthy pregnant, non-pregnant and postpartum measurements of the included studies were plotted, along with the $5^{\text {th }}$ and $95^{\text {th }}$ percentile calculated from intra-study variances (Figure 4). Each point estimate is indexed as a value from a LQ, MQ of HQ study as stipulated by study quality assessment.

Figure 7 illustrates reference values for BAD throughout physiological, uncomplicated, pregnancy, similarly to FMD in uncomplicated pregnancies, with the absolute course by means of the mean, $5^{\text {th }}$ and $95^{\text {th }}$ percentile of both non-pregnant, healthy pregnant and postpartum measurements, portraying intra-study variances.

\section{BRACHIAL ARTERY DIAMETER}

When compared to reference conditions, BAD increased significantly, in a progressive manner, during the intervals 22 - 28, 29 - 35 and 36 - 41 weeks' gestation with values of, respectively, $0.13 \mathrm{~mm}(0.0-0.2 \mathrm{~mm})$ relative $4.2 \%(0.9-7.4 \%), 0.20 \mathrm{~mm}(0.1-0.3 \mathrm{~mm})$ relative $6.5 \%(4.8-8.4 \%)$, and $0.24 \mathrm{~mm}(0.1-0.4 \mathrm{~mm})$ relative $7.7 \%(2.9-12.8 \%)$. Forest plots of BAD course throughout uncomplicated pregnancies and pregnancies by GHD compared to the reference value are depicted in Figure 5 and Figure 6, respectively. Funnel plot asymmetry was detected at 22 to 28 weeks' gestational age in healthy pregnancies ( $p$ $=0.03$ ). However, the pooled estimate remained $0.13 \mathrm{~mm}$ after correction, using the trim and fill method ${ }^{50}$. No other funnel plot asymmetry was found $(0.38<p<0.77)$.

In pregnancies complicated by GHD brachial artery diameter significantly increased by $0.36 \mathrm{~mm}(0.22-0.49 \mathrm{~mm})$ relative $11.6 \%(7.1-15.8 \%)$ at 29 - 35 weeks of gestation ${ }^{20,47}$, when compared to reference measures. No significant differences in BAD measurements were detected between healthy pregnancies and pregnancies complicated by GHD in this specific interval $(p=0.25)$. 


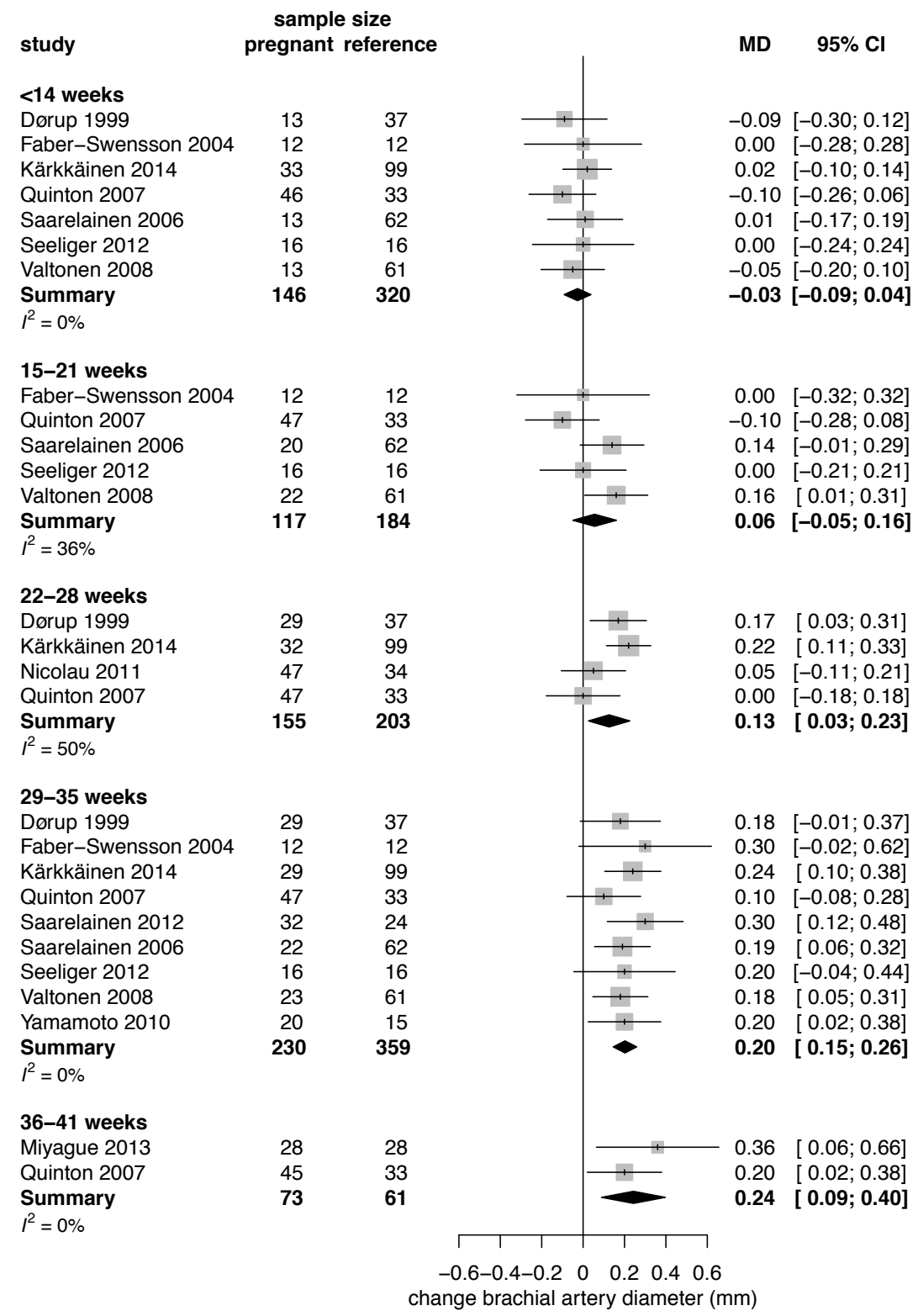

Figure 5 Forest plot of brachial artery diameter (BAD) during physiologic pregnancies at $<14$ weeks, 15 - 21 weeks, 22 - 28 weeks, 29 - 35 weeks, and 36 - 41 weeks' gestation compared to reference. BAD is reported as mean difference (MD) between pregnant and reference BAD, with $95 \% \mathrm{Cl}$, in millimetres (mm). 


\begin{tabular}{lrr} 
Study & \multicolumn{2}{c}{$\begin{array}{c}\text { Sample size } \\
\text { Pregnant Referen }\end{array}$} \\
29-35 weeks & & \\
Saarelainen 2012 & 26 & 24 \\
Yamamoto 2010 & 20 & 15 \\
Summary & 46 & $\mathbf{3 9}$ \\
$I^{2}=0 \%$ & &
\end{tabular}

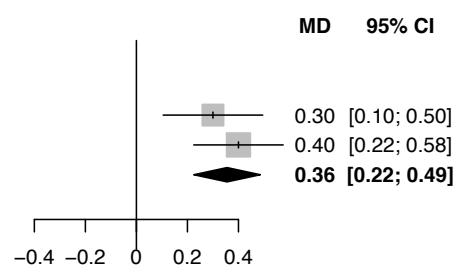

Change brachial artery diameter $(\mathrm{mm})$

Figure 6 Forest plot of brachial artery diameter (BAD) during pregnancies complicated by gestational hypertensive disease at 29 - 35 weeks' gestation compared to reference. BAD is reported as mean difference (MD) between pregnant and reference BAD, with $95 \% \mathrm{Cl}$, in millimetres $(\mathrm{mm})$.

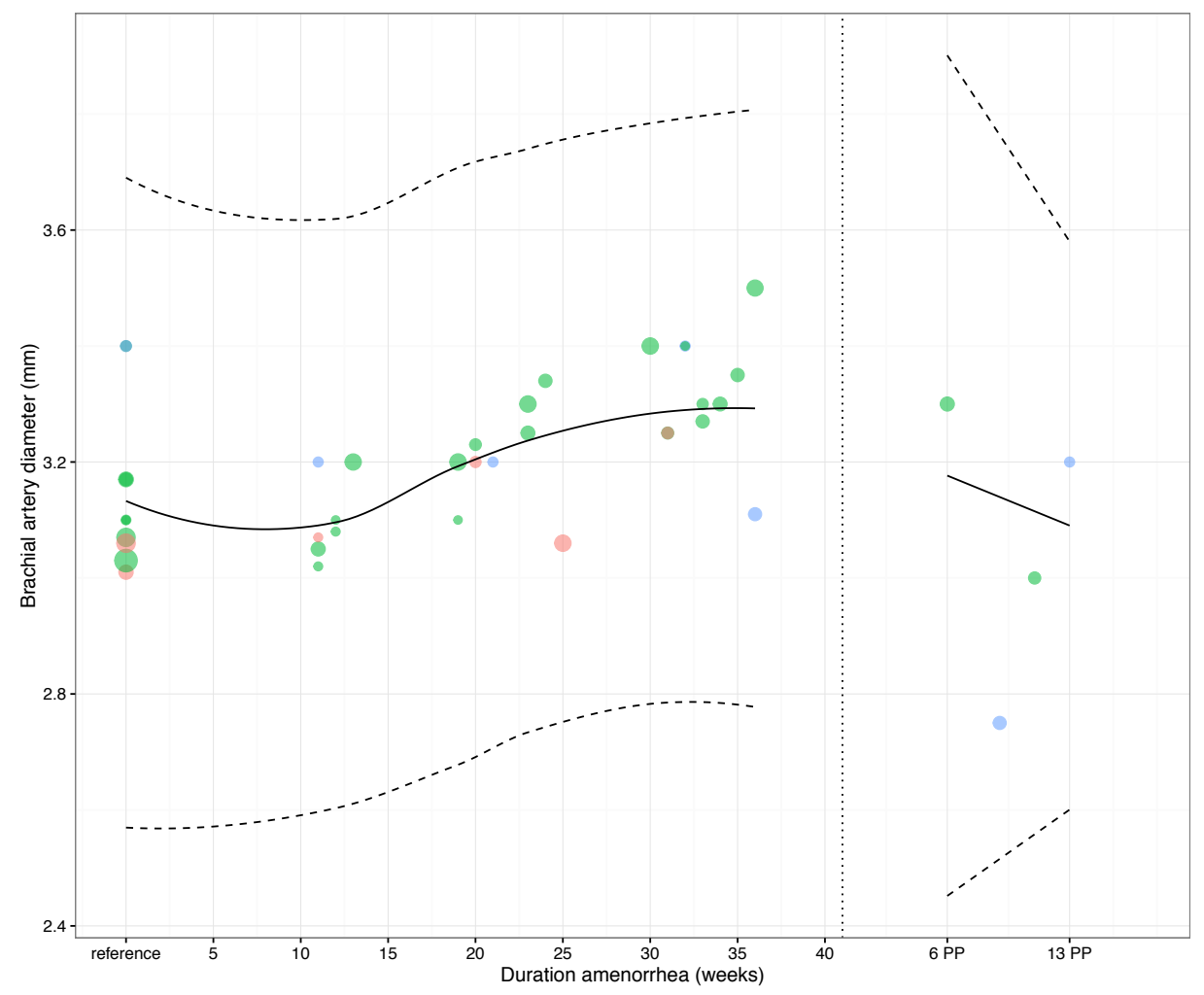

Figure 7 Brachial artery diameters in millimetres $(\mathrm{mm})$ during physiologic pregnancies and postpartum (PP). The circle size indicates the sample size of the point estimate. The colour of the circle refers to the quality assigned to the study: red $=\mathrm{LQ}$, green= MQ, blue $=\mathrm{HQ}$. Curve fit is weighted by the inverse variance and plotted with $5^{\text {th }}$ and $95^{\text {th }}$ percentiles. The $50^{\text {th }}$ percentile is represented by a solid line. 


\section{DISCUSSION}

In this meta-analysis we systematically reviewed the adaptation of FMD and BAD throughout pregnancy. In uncomplicated pregnancies FMD was increased in the second and third trimester. BAD increased progressively in a steady manner, by the second trimester, but did not reach significance in the first half of the second trimester. In pregnancies complicated by GHD, both BAD and FMD were not significantly different compared to healthy pregnancies.

Pregnancy is characterized by profound vasodilatation ${ }^{51}$. Throughout gestation extensive hemodynamic adaptations evolve, by way of plasma expansion and increase in cardiac output, instigated by an abrupt and profound reduction in peripheral vascular resistance ${ }^{51}$. These adaptations cause an increase in shear stress, on the vascular endothelium, thereby triggering vascular reactivity to self-regulate tone. In contrast, pregnancy induced haemodilution and a decreased blood viscosity lowers shear forces upon the endothelium 52. Subsequently, local endothelial mechanisms induce a vasomotor response by means of vasodilatation in functionally intact endothelium, as observed in conduit arteries, designated as FMD ${ }^{24,53}$. The exact system for detecting an increase in shear stress is thought to be related to calcium-activated potassium channels that react by shifting to an open conformation, hyperpolarizing the endothelial cell and subsequently causing calcium to enter the cell $24,54-57$. As such, calcium activates endothelial nitric oxide synthase leading to NO synthesis.

Physiologically, adequate endothelial function exhibits balance and local regulation of anti-inflammatory-, antithrombotic-, anticoagulant-, profibrinolytic-, antihypertrophic- and endothelium-dependent vasodilatation characteristics ${ }^{58-60}$. A dysfunctional endothelium can be non-invasively evaluated by assessing the endothelium dependent vasodilatation, a measurement also feasible during pregnancy. The change in vasodilator response during pregnancy may be reflected by an increase in FMD. We observed that additional to a progressively increasing BAD, FMD is increased in the second half of pregnancy, a finding in line with several longitudinal studies ${ }^{15}$.

The fact that FMD does not steadily rise throughout gestation could be the result of the progressively increasing BAD. Considering that smaller vessels have a more profound FMD response to a stimulus than larger vessels do, it is striking than an increased FMD is seen coinciding with an increased BAD during pregnancy ${ }^{61-63}$. Moreover, when stretched, the elastic properties of the same vessel respond differently, usually towards more stiffening. Interestingly, even though vascular function is increased during pregnancy, we found no indication that vessel structure is permanently changed, as both function and structure 
return to similar levels after pregnancy. This is in contrast to studies indicating that an increased function could result in structural adaptation ${ }^{64,65}$. As mentioned, the increase in flow-mediated response is directly proportional to baseline diameter, making it baseline diameter dependent. An allometric model involving FMD (on a log scale) as the outcome and BAD (log-transformed) as a covariate has been proposed to resolve this dependency 66. However, we could not investigate this dependency in this meta-analysis. Besides mechanical effects, increased gestation-induced low-grade inflammation may also affect $\mathrm{FMD}$, as the maternal inflammatory response in late pregnancy is suggested to relate to changes in FMD due to diminished NO-mediated dilatation ${ }^{19}$.

Maladaptation of maternal vascular and central hemodynamic function in pregnancy may affect remodelling of arteries and with it trophoblastic invasion, creating an imbalance in maternal-foetal interface. These adverse adjustments may result in additional endothelial impairment. Clinically this impairment manifests in placental dysfunction and induced foetal growth restriction on the one hand, and maternal capillary-leak induced oedema, organ hypoperfusion, hypertension, proteinuria, platelet activation and consumption and erythrocyte decay on the other hand ${ }^{67,68}$. Recently a large meta-analysis reported a lower FMD before the clinical onset and during preeclampsia compared to uncomplicated pregnancies ${ }^{69}$. We were unable to confirm their findings, mainly due to our different objective for which we included only studies using a non-pregnant reference value within the same study. However, when we compare mean FMD values of the studies included in that review to the reference values computed by our study, they are all below the $50^{\text {th }}$ percentile and, more than half below the $25^{\text {th }}$ percentile reference line.

Pitfalls and discrepancies that could have resulted in the dispersal of non-pregnant FMD values can be found in methodological shortcomings of included studies. According to Weissgerber et el. ${ }^{21}$ continuous FMD diameter measurements are required to adjust for delayed peak dilatation in pregnancy. Since post-release diameter at 60 seconds differs significantly from non-pregnant women who peak on average at 45 seconds. Other methodological shortcomings within studies such as caffeine use, menstrual phase used as reference, fasted state and adequate acclimatization could also be responsible for the broad interval between the $5^{\text {th }}$ and $95^{\text {th }}$ percentiles. With reference to the detected funnel plot asymmetry in physiological pregnancies during gestational age 22 - 28 week, the results should be interpreted with caution. Even though the trim and fill method provided an identical estimate, no presuppositions should be drawn. Considering that the mechanism for publication bias is unknown and rationales other than this specific bias could have elicited funnel plot asymmetry ${ }^{31}$. According to Egger et el., the capacity to ascertain publication bias is restricted when meta-analysis consist solely of limited and/ or small studies, and in this case both ${ }^{32}$. 
Limitations of this study should be acknowledged. First, our meta-analysis was based on a relatively limited number of studies, mostly due to the necessity of a non-pregnant reference measurement within the studied population. This could have resulted in a lower statistical power because of numbers, but increased validity because of the use of identical measurement techniques. Second, authors reported participant's anthropometric data insufficiently and infrequently. It was therefore impossible to analyse the contribution of these variables to the heterogeneity observed. Third, methodological shortcomings within and disparities amidst studies have limited data interpretation, which is reflected by our meta-regression analysis that exhibited a significant effect on the regression when study quality was taken into account. Groups overlap due to the combination of extensive spreading of non-pregnant FMD values and broad reference interval of pregnant women. This implies that, in clinical practice, no conclusion can be drawn based on a single FMD measurement, but serial assessments within one individual may detect alterations in individuals' endothelial function that could be of added value in clinical practice to identify women with deviant endothelial function.

\section{CONCLUSION}

This review and meta-analysis was systematically conducted to summarize and evaluate current literature on the extent of physiologic and pathological endothelial adaptation throughout pregnancy. During healthy pregnancy endothelial dependent vasodilatation and brachial artery diameter are increased. Women with a complicated pregnancy fell within the lower range of FMD values when compared with uncomplicated pregnancies, but as group, did not differ from each other. A lack of serial assessments impeded an adequate comparison with the course of adaptation of uncomplicated pregnancy. 


\section{REFERENCES}

1. Mahendru AA, Everett TR, Wilkinson IB, Lees CC, McEniery CM. A longitudinal study of maternal cardiovascular function from preconception to the postpartum period. J. Hypertens. Apr 2014;32(4):849-856.

2. ClappJF, Capeless E. Cardiovascular Function Before, During, and After the First and Subsequent Pregnancies. Am. J. Cardiol. 1997;80:1469-1473.

3. Grindheim G, Estensen ME, Langesaeter E, Rosseland LA, Toska K. Changes in blood pressure during healthy pregnancy: a longitudinal cohort study. J. Hypertens. Feb 2012;30(2):342-350.

4. deAlvarez RR, Wash S. Glomerular filtration rates, renal plasma flow, and sodium and water retention in pregnancy toxemia. Am. J. Obstetrics and Gynaecology. Dec 1950;60(5):1051-1067.

5. Pritchard JA. Changes in the blood volume during pregnancy and delivery. Anesthesiology. 1965;26(4):393399.

6. Chesley LC. Plasma and red cell volumes during pregnancy. Am. J. Obstet. Gynecol. Feb 01 1972;112(3):440450.

7. Bader RA, Bader ME, Rose DF, Braunwald E. Hemodynamics at rest and during exercise in normal pregnancy as studies by cardiac catheterization. J. Clin. Invest. Oct 1955;34(10):1524-1536.

8. Robson SC, Hunter S, Boys RJ, Dunlop W. Serial study of factors influencing changes in cardiac output during human pregnancy. Am. J. Physiol. 1989;256(25):1060-1065.

9. Hunter S, Robson SC. Adaptation of the maternal heart in pregnancy. Br. Heart J. 1992;68:540-543.

10. Mabie WC, DiSessa TG, Crocker LG, Sibai BM, Arheart KL. A longitudinal study of cardiac output in normal human pregnancy. Am. J. Obstet. Gynecol. Mar 1994;170(3):849-856.

11. Haynes MP, Sinha D, Russell KS, et al. Membrane Estrogen Receptor Engagement Activates Endothelial Nitric Oxide Synthase via the PI3-Kinase-Akt Pathway in Human Endothelial Cells. Circ Res. . 2000;87:677-682.

12. Simoncini T, Hafezi-Moghadam A, Brazil DP, Ley K, Chin WW, Liao JK. Interaction of oestrogen receptor with the regulatory subunit of phosphatidylinositol-3-OH kinase. Nature. Sep 28 2000;407(6803):538-541.

13. Boeldt DS, Bird IM. Vascular adaptation in pregnancy and endothelial dysfunction in preeclampsia. J. Endocrinol. Jan 2017;232(1):R27-R44.

14. Bird IM, Zhang L, Magness RR. Possible mechanisms underlying pregnancy-induced changes in uterine artery endothelial function. Am. J. Physiol. Regul. Integr. Comp. Physiol. Feb 2003;284(2):R245-258.

15. Quinton AE, Cook CM, Peek MJ. A longitudinal study using ultrasound to assess flow-mediated dilatation in normal human pregnancy. Hypertens. Pregnancy. 2007;26(3):273-281.

16. Dorup I, Skajaa K, Sorensen KE. Normal pregnancy is associated with enhanced endothelium-dependent flow-mediated vasodilation. Am. J. Physiol. 2. 1999;276: H821-H825.

17. Lopez-Jaramillo P, Garcia RG, Lopez M. Preventing pregnancy-induced hypertension: are there regional differences for this global problem? J. Hypertens. Jun 2005;23(6):1121-1129.

18. Corretti MC, Anderson TJ, Benjamin EJ, et al. Guidelines for the ultrasound assessment of endothelialdependent flow-mediated vasodilation of the brachial artery: a report of the International Brachial Artery Reactivity Task Force. Journal of the American College of Cardiology. Jan 16 2002;39(2):257-265.

19. Flo K, Blix ES, Husebekk A, et al. A longitudinal study of maternal endothelial function, inflammatory response and uterine artery blood flow during the second half of pregnancy. Acta Obstet. Gynecol. Scand. Feb 2016;95(2):225-232.

20. Saarelainen $\mathrm{H}$, Karkkainen $\mathrm{H}$, Valtonen $\mathrm{P}$, et al. Flow-mediated vasodilation is not attenuated in hypertensive pregnancies despite biochemical signs of inflammation. ISRN Obstet. Gynecol. 2012;2012:709464.

21. Weissgerber TL, Davies GA, Tschakovsky ME. Brachial artery flow-mediated dilation is not affected by pregnancy or regular exercise participation. Clin. Sci. (Lond.). Oct 2011;121(8):355-365.

22. Hamad R, Eriksson MJ, A S, Hamsten A, Bremmea K. Decreased flow-mediated dilation is present 1 year after a pre-eclamptic pregnancy. J. Hypertens. 2007;25(2301-2307).

23. Harris RA, Nishiyama SK, Wray DW, Richardson RS. Ultrasound assessment of flow-mediated dilation. Hypertension. May 2010;55(5):1075-1085.

24. Miura H, Wachtel RE, Liu Y, et al. Flow-Induced Dilation of Human Coronary Arterioles Important Role of Ca2 -Activated K Channels Circulation. 2001;103:1992-1998.

25. Uehata A, Lieberman EH, Gerhard MD, et al. Noninvasive assessment of endothelium-dependent flowmediated dilation of the brachial artery. Vasc. Med. 1997;2(2):87-92. 
26. Deanfield JE, Halcox JP, Rabelink TJ. Endothelial function and dysfunction: testing and clinical relevance. Circulation. Mar 13 2007;115(10):1285-1295.

27. Esper RJ, Nordaby RA, Vilarino JO, Paragano A, Cacharron JL, Machado RA. Endothelial dysfunction: a comprehensive appraisal. Cardiovasc. Diabetol. Feb 23 2006;5:4

28. Hayden JA, L.Cartwright DAvdWJ, Cote P, Bombardier C. Assessing Bias in Studies of Prognostic Factors. Ann. Intern. Med. 2013;158:280-286.

29. Abudu OO, Sofola OA. Plasma volume in normal pregnant Nigerian primigravidae. Int. J. Gynaecol. Obstet. Apr 1985;23(2):137-142.

30. DerSimonian R, Laird N. Meta-Analysis in Clinical Trials. Control. Clin. Trials. 1986;7:177-188.

31. Higgins J, Green S. Cochrane Handbook for Systematic Reviews of Interventions Version 5.1.0 [updated March 2011]. Cochrane Collab 2011, :Available from http//handbook.cochrane.org.

32. Egger M, Smith GD, Schneider M, Minder C. Bias in meta-analysis detected by a simple, graphical test. Br. Med. J. 1997;316:629-634

33. Seeliger C, Brueckmann A, Schleußner E. Maternale Endothelfunktion in Schwangerschaft und Wochenbett - ultraschallgestützte longitudinale Erfassung mittels FlowMediated Dilatation (FMD). Z. Gastroenterol. Mar 2012;50(3):271-272.

34. Miyague AH, Martins WP, Machado JS, et al. Maternal flow-mediated dilation and nitrite concentration during third trimester of pregnancy and postpartum period. Hypertens. Pregnancy. Aug 2013;32(3):225-234.

35. Higgins J, Thompson SG, Deeks JJ, Altman DG. Measuring inconsistency in meta-analyses. Br. Med. J. 2003;327:557-560.

36. Team TRC. R: A Language and Environment for Statistical Computing. 2015.

37. Schwarzer G. Meta: General package for meta-analysis. R package version 4.3-2 2015

38. Weissgerber TL, Davies G a L, ME. T. Low flow-mediated constriction occurs in the radial but not the brachial artery in healthy pregnant and nonpregnant women. J. Appl. Physiol. 2010;108(5):1097-1105.

39. Noori M, Donald AE, Hingorani AD, Williams DJ. Gestational vasoconstriction of the brachial artery at low-flow; a novel observation that suggests shear stress has a primary role in the vasodilatation of healthy pregnancy. Archives of Disease in Childhood - Fetal and Neonatal Edition. 2011;96(Suppl 1):Fa97-Fa97.

40. Floka S, Barabashkina A. The cardiovascular system condition and the kidney function in pregnant women with hypertension. Pregnancy Hypertension. Conference: 17th World Congress of the International Society for the Study of Hypertension in Pregnancy, ISSHP. 2010.

41. Mori T, Watanabe K, Iwasaki A, et al. Differences in vascular reactivity between pregnant women with chronic hypertension and preeclampsia. Hypertens. Res. Feb 2014;37(2):145-150.

42. Andolf E, Kahan $T$, lacobaeus $C$, Jörneskog $G$, Thorsell M, Bremme K. The vascular tree during pregnancy and at follow-up - A longitudinal study of maternal vascular function. Pregnancy Hypertens. 2013;3(2):67.

43. Brückmann A, Seeliger Ch, E S. Prediction of preeclampsia with flow-mediated-dilatation in early pregnancy and evaluation of endothelial function during pregnancy and postpartum.

44. Yoshida A, Nakao S, Kobayashi H, Kobayashi M. Noninvasive Assessment of Flow-Mediated Vasodilation With 30-MHz Transducer in Pregnant Women. Hypertension. 1998;31(1200-1201).

45. Alfredo M Germain, Verónica Irribarra, Gloria Valdés, et al. Ultrasound assessment of endothelial function in Chilean children and adults. Rev Méd Chile 2004(132):437-444.

46. Nicolau LG, Martins WP, Gallarreta FM, Lima JC, Filho FM. Influence of pregnancy and smoking on brachial artery flow-mediated dilation values and time until maximum response. Arch. Gynecol. Obstet. Aug 2011;284(2):313-317.

47. Yamamoto T, Suzuki Y, Kojima K, Suzumori N, Suzuki T. The biological investigation of prostacyclin in preeclamptic women seen reduced endothelial function. Hypertens. Pregnancy. 2010;29(4):484-491.

48. Hamad RR, Eriksson MJ, Berg E, Larsson A, Bremme K. Impaired endothelial function and elevated levels of pentraxin 3 in early-onset preeclampsia. Acta Obstet. Gynecol. Scand. Jan 2012;91(1):50-56.

49. Saarelainen H, Tomi Laitinen, Raitakari OT, et al. Pregnancy-Related Hyperlipidemia and Endothelial Function in Healthy Women. Circ. J. 2006;70:768-772.

50. Duval S, Tweedie R. Trim and Fill: A Simple Funnel-Plot-Based Method of Testing and Adjusting for Publication Bias in Meta-Analysis. Biometrics. 2000;56:455-463.

51. Bosio PM, McKenna PJ, Conroy R, O'Herlihy C. Maternal central hemodynamics in hypertensive disorders of pregnancy. Obstet. Gynecol. Dec 1999;94(6):978-984.

52. Quyyumi AA. Endothelial Function in Health and Disease: New Insights into the Genesis of Cardiovascular Disease. Am J Med. 1998;105:32-39. 
53. Lie M, Sejersted OM, Kiil F. Local Regulation of Vascular Cross Section during Changes in Femoral Arterial Blood Flow in Dogs. CircaUuon Rtitmcb. 1970;27:727-737.

54. Pohl U, Holtz J, Busseand R, Bassenge E. Crucial Role of Endothelium in the Vasodilator Response to Increased Flow in Vivo. Hypertension. 1986;8:37-44.

55. Olesen SP, Clapham DE, Davies PF. Haemodynamic shear stress activates a K+ current in vascular endothelial cells. Nature. Jan 14 1988;331(6152):168-170.

56. Cooke JP, Jr. ER, Andon NA, Loscalzo J, Dzau VJ. Flow Activates an Endothelial Potassium Channel to Release an Endogenous Nitrovasodilator. J. Clin. Invest. 1991;88:1663-1671.

57. Joannides R, Haefeli WE, Linder L, et al. Nitric-Oxide Is Responsible for Flow-Dependent Dilatation of Human Peripheral Conduit Arteries in-Vivo. Circulation. Mar 1 1995;91(5):1314-1319.

58. Nathan C. Points of control in inflammation. Nature. 2002;420:846-852.

59. Pearson JD. Endothelial cell function and thrombosis. Baillieres Best Pract. Res. Clin. Haematol. Sep 1999;12(3):329-341.

60. Furchgott RF, Zawadzki JV. The obligatory role of endothelial cells in the relaxation of arterial smooth muscle by acetylcholine. Nature. Nov 27 1980;288(5789):373-376.

61. Herrington DM, Fan L, Drum M, et al. Brachial flow-mediated vasodilator responses in population-based research: methods, reproducibility and effects of age, gender and baseline diameter. J. Cardiovasc. Risk. Oct 2001;8(5):319-328.

62. Anderson TJ, Uehata A, Gerhard MD, et al. Close Relation of Endothelial Function in the Human Coronary and Peripheral Circulations. JACC 1995;26(5):1235-1241.

63. David S. Celemajer, Keld E Sorsensen, Vanda M Gooch, et al. Non-invasive detection of endothelial dysfunction in children and adults at risk of atherosclerosis. The Lancet. 1992;340:1111-1115.

64. Thijssen DH, Carter SE, Green DJ. Arterial structure and function in vascular ageing: are you as old as your arteries? J. Physiol. Apr 15 2016;594(8):2275-2284.

65. Scholten RR, Thijssen DJ, Lotgering FK, Hopman MT, Spaanderman MEA. Cardiovascular effects of aerobic exercise training in formerly preeclamptic women and healthy parous control subjects. Am. J. Obstet. Gynecol. Nov 2014;211(5):516 e511-516 e511.

66. Atkinson G, Batterham AM. The clinical relevance of the percentage flow-mediated dilation index. Curr. Hypertens. Rep. Feb 2015;17(2):4.

67. Stella $\mathrm{CL}$, Sibai BM. Preeclampsia: Diagnosis and management of the atypical presentation. J. Matern. Fetal Neonatal Med. Jul 2006;19(7):381-386.

68. Report of the National High Blood Pressure Education Program Working Group on High Blood Pressure in Pregnancy. Am. J. Obstet. Gynecol. 2000;183(1):s1-s22.

69. Weissgerber TL, Milic NM, Milin-Lazovic JS, Garovic VD. Impaired Flow-Mediated Dilation Before, During, and After Preeclampsia A Systematic Review and Meta-Analysis. Hypertension. Feb 2016;67(2):415-423.

70. Faber-Swenson A, O'Callaghan S, Walters W. Endothelial cell function enhancement in a late normal human pregnancy. Aust. N. Z. J. Obstet. Gynaecol. 2004(44):525-529.

71. Kärkkäinen $H$, Saarelainen $H$, Valtonen $P$, et al. Carotid artery elasticity decreases during pregnancy - the Cardiovascular Risk in Young Finns study. BMC Pregnancy Childbirth. 2014;14(98).

72. Valtonen P, Laitinen T, Lyyra-Laitinen T, et al. Serum L-Homoarginine Concentration is Elevated During Normal Pregnancy and is Related to Flow-Mediated Vasodilatation. Circ. J. 2008;72:1879-1884. 


$\begin{array}{ll}\text { Component } & \text { PubMed } \\ \text { Pregnancy } & \text { "Pregnancy" [Mesh] OR "pregnancy" [Title/Abstract] OR "pregnancies" [Title/ } \\ & \text { Abstract] OR "pregnant" [Title/Abstract] OR "gestation" [Title/Abstract] OR } \\ & \text { "gestations" [Title/Abstract] OR "gestational" [Title/Abstract] OR "gravidity" } \\ & \text { [Mesh] OR "gravidity" [Title/Abstract] OR "gravidities" [Title/Abstract] OR "gravid" } \\ & \text { [Title/Abstract] } \\ \text { Pregnancy- } & \text { "Hypertension, pregnancy-induced" [Mesh] OR "pregnancy induced } \\ \text { induced } & \text { hypertension" [Title/Abstract] OR "pregnancy associated hypertension" [Title/ } \\ \text { hypertension } & \text { Abstract] OR "PIH" [Title/Abstract] OR "hypertensive pregnancy" [Title/Abstract] } \\ & \text { OR "pregnancy hypertension" [Title/Abstract] OR "gestational hypertension" } \\ & \text { [Title/Abstract] OR "HELLP syndrome" [Mesh] OR "HELLP" [Title/Abstract] OR } \\ & \text { "Hemolysis, Elevated Liver Enzymes, Lowered Platelets" [Title/Abstract] }\end{array}$

$\begin{array}{ll}\text { Fetal growth } & \text { "Fetal Growth Retardation" [Mesh] OR "foetal growth retardation" [Title/ } \\ \text { retardation } & \text { Abstract] OR "fetal growth restriction" [Title/Abstract] OR "FGR" [Title/Abstract] } \\ & \text { OR "intrauterine growth retardation" [Title/Abstract] OR "intrauterine growth } \\ & \text { restriction" [Title/Abstract] OR "IUGR" [Title/Abstract] OR "Infant, Small for } \\ & \text { Gestational Age" [Mesh] OR "small for gestational age" [Title/Abstract] OR "SGA" } \\ & \text { [Title/Abstract] } \\ \text { Preeclampsia } & \text { "Pre-eclampsia" [Mesh] OR "pre-eclampsia" [Title/Abstract] OR "preeclampsia" } \\ & \text { [Title/Abstract] OR "preeclamptic" [Title/Abstract] OR "pre-eclamptic" [Title/ } \\ & \text { Abstract] or "PE" [Title/Abstract] OR "Eclampsia" [Mesh] OR "eclampsia" [Title/ } \\ & \text { Abstract] OR "eclampsias" [Title/Abstract] OR "eclamptic" [Title/Abstract] OR } \\ & \text { "toxemia" [Title/Abstract] OR "toxemias" [Title/Abstract] }\end{array}$
Gestational "Diabetes, Gestational" [Mesh] OR "pregnancy induced diabetes" [Title/ diabetes Abstract] OR "gestational diabetes" [Title/Abstract] OR "diabetes gravidarum" [Title/abstract]

Flow mediated "Brachial Artery" [MeSH] OR "Endothelium, Vascular" [MeSH] OR "Vasodilation" dilatation $\quad[\mathrm{MeSH}] \mathrm{OR}$ "FMD" [Title/Abstract] OR "flow mediated" [Title/Abstract] OR "flow mediated vasodilatation" [Title/Abstract] OR "flow mediated vasodilation" [Title/ Abstract] OR "flow mediated dilatation" [Title/Abstract] OR "flow mediated dilation" [Title/Abstract] OR "flow dependent" [Title/Abstract] OR "flow dependent vasodilatation" [Title/Abstract] OR "flow dependent vasodilation" [Title/Abstract] OR "flow dependent dilatation" [Title/Abstract] OR "flow dependent dilation" [Title/Abstract] OR "brachial artery" [Title/Abstract] OR "brachial artery vasodilatation" [Title/Abstract] OR "brachial artery vasodilation" [Title/Abstract] OR "brachial artery dilatation" [Title/Abstract] OR "brachial artery dilation" [Title/Abstract] OR "brachial artery reactivity" [Title/Abstract] OR "endothelium dependent" [Title/Abstract] OR "endothelium dependent vasodilatation" [Title/Abstract] OR "endothelium dependent vasodilation" [Title/Abstract] OR "endothelium dependent dilatation" [Title/Abstract] OR "endothelium dependent dilation" [Title/Abstract] OR "endothelium mediated" [Title/Abstract] OR "endothelium mediated vasodilatation" [Title/Abstract] OR "endothelium mediated vasodilation" [Title/Abstract] OR "endothelium mediated dilatation" [Title/Abstract] OR "endothelium mediated dilation" [Title/ Abstract] OR "shear stress" [Title/Abstract] 


\section{Embase}

exp pregnancy/ or exp gravidity/ or exp gestation/ or (pregnancy or pregnancies or pregnant or gestation or gestations or gestational or gravidity or gravidities or gravid).ti,ab.

exp maternal hypertension/ or exp HELLP syndrome/ or (pregnancy induced hypertension or pregnancy associated hypertension or PIH or hypertensive pregnancy or pregnancy hypertension or gestational hypertension or HELLP or Hemolysis, Elevated Liver Enzymes, Lowered Platelets).ti,ab.

exp intrauterine growth retardation/ or exp small for date infant/ or (fetal growth retardation or fetal growth restriction or FGR or intrauterine growth retardation or intrauterine growth restriction or IUGR or small for gestational age or SGA ).ti,ab.

exp preeclampsia/ or exp eclampsia/ or (pre-eclampsia or preeclampsia or pre-eclamptic or preeclamptic or PE or eclampsia or eclampsias or eclamptic or toxemia or toxemias).ti,ab.

exp pregnancy diabetes mellitus/ or (pregnancy induced diabetes or gestational diabetes or diabetes gravidarum).ti,ab.

FMD or flow mediated or flow mediated vasodilatation or flow mediated vasodilation or flow mediated dilatation or flow mediated dilation or flow dependent or flow dependent vasodilatation or flow dependent vasodilation or flow dependent dilatation or flow dependent dilation or brachial artery or brachial artery vasodilatation or brachial artery vasodilation or brachial artery dilatation or brachial artery dilation or brachial artery reactivity or endothelium dependent or endothelium dependent vasodilatation or endothelium dependent vasodilation or endothelium dependent dilatation or endothelium dependent dilation or endothelium mediated or endothelium mediated vasodilatation or endothelium mediated vasodilation or endothelium mediated dilatation or endothelium mediated dilation or shear stress).ti,ab. 


\begin{tabular}{|c|c|c|c|c|c|c|c|c|}
\hline \multirow{3}{*}{ Study } & \multicolumn{8}{|c|}{ Non pregnant } \\
\hline & \multicolumn{2}{|c|}{ Subjects (n) } & \multicolumn{2}{|c|}{ Age (years) } & \multicolumn{2}{|c|}{ Weight (kg) } & \multicolumn{2}{|c|}{ Height (cm) } \\
\hline & $\mathrm{R}$ & $\mathrm{P}$ & $\mathrm{R}$ & $\mathrm{P}$ & $\mathrm{R}$ & $\mathrm{P}$ & $\mathrm{R}$ & $\mathrm{P}$ \\
\hline Dørup 1999 & 37 & 71 & 29.7 & 30.4 & - & - & - & - \\
\hline Faber-Swensson $2004^{70}$ & 12 & 12 & - & - & - & - & - & - \\
\hline Germain 2004 & 24 & 8 & 33.3 & 29.9 & - & - & - & - \\
\hline Hamad 2012 & 65 & 30 & 31 & 31 & - & - & 167 & 167 \\
\hline Kärkkäinen $2014{ }^{71}$ & 99 & 94 & 32.2 & 31.9 & 68.4 & - & 166.3 & 166.2 \\
\hline Miyague 2013 & 28 & 28 & 23.7 & 23.7 & - & - & - & - \\
\hline Nicolau 2011 & 34 & 47 & 25.3 & 25.3 & - & - & - & - \\
\hline Quinton 2007 & 33 & 47 & 28.4 & 28.4 & - & - & 165 & 165 \\
\hline Saarelainen 2006 & 62 & 55 & 30.7 & 30.7 & 67 & - & 167 & 166 \\
\hline Saarelainen 2012 & 24 & 32 & 31 & 31 & - & - & - & - \\
\hline Seeliger 2012 & 16 & 16 & 30.6 & 30.6 & 63.2 & 63.2 & 165.5 & 165.5 \\
\hline Valtonen 200872 & 61 & 58 & 31 & 31 & 66.9 & - & 168 & 165 \\
\hline Weissgerber 2011 & 28 & 24 & 32.8 & 31.3 & - & - & - & - \\
\hline Yamamoto 2010 & 15 & 20 & 30 & 30 & - & - & - & - \\
\hline
\end{tabular}

Table 2 Characteristics of included studies for physiological pregnancies, arranged by first author and year of publication. Characteristics are presented by means reported by the study. Parity is in italic. Parity/gravity: Nulli, Primi, Multi. Reference (R) flow mediated dilation was measured in non-pregnant controls (NP) or postpartum (PP). During physiologic pregnancy (P), flow mediated dilation was measured at different durations of amenorrhea.

\begin{tabular}{|c|c|c|c|c|c|c|c|c|}
\hline \multirow{3}{*}{ Study } & & & \multicolumn{6}{|c|}{ Non-pregnant } \\
\hline & \multicolumn{2}{|c|}{ Subjects (n) } & \multicolumn{2}{|c|}{ Age (years) } & \multicolumn{2}{|c|}{ Weight (kg) } & \multicolumn{2}{|c|}{ Height (cm) } \\
\hline & $\mathrm{R}$ & GHD & $\mathrm{R}$ & GHD & $\mathrm{R}$ & GHD & $\mathrm{R}$ & GHD \\
\hline Hamad 2012 & 33 & 33 & 31 & 31 & - & - & 166 & 166 \\
\hline Saarelainen 2012 & 24 & 26 & 31 & 31 & - & - & - & - \\
\hline Yamamoto 2010 & 15 & 20 & 30 & 30 & - & - & - & - \\
\hline
\end{tabular}

Table 3 Characteristics of included studies, gestational hypertensive disorder pregnancies, arranged by first author and year of publication. Characteristics are presented by means reported by the study. Parity is in italic. Parity/gravity: Nulli, Primi, Multi. Reference (R) flow mediated dilation was measured in non-pregnant controls (NP) or postpartum (PP). During gestational hypertensive disorder pregnancies (GHD) flow mediated dilation was measured at 34 and 35 weeks of amenorrhea. 


\begin{tabular}{|c|c|c|c|c|c|c|c|}
\hline \multicolumn{8}{|c|}{ Parity / gravidity (n) } \\
\hline \multicolumn{2}{|c|}{ Nulli } & \multicolumn{2}{|c|}{ Primi } & \multicolumn{2}{|c|}{ Multi } & \multirow[t]{2}{*}{ Reference } & \multirow{2}{*}{$\begin{array}{l}\text { Duration of amenorrhea at FMD } \\
\text { measurement (weeks) }\end{array}$} \\
\hline $\mathrm{R}$ & $\mathrm{P}$ & $\mathrm{R}$ & $P$ & $\mathrm{R}$ & $\mathrm{P}$ & & \\
\hline- & - & - & - & - & - & NP & $12,24,35$ \\
\hline- & - & - & - & - & - & NP & $12,19,32$ \\
\hline- & - & - & - & - & - & NP & $14-15,24-27+6,32-38+6$ \\
\hline & & 30 & 30 & & & PP (3 months) & 33 \\
\hline- & - & - & - & - & - & NP & $11,23,33$ \\
\hline- & - & 16 & 16 & 12 & 12 & PP (10 weeks) & 36 \\
\hline- & - & - & - & - & - & NP & 25 \\
\hline- & - & - & - & - & - & PP (7 weeks) & $13,19,23,30,36$ \\
\hline- & - & - & - & - & - & NP & $<14,15-27,>28$ \\
\hline 18 & 18 & - & - & - & - & PP (3 months) & 34 \\
\hline- & - & - & - & - & - & PP (14 weeks) & $11,21,32$ \\
\hline- & - & - & - & - & - & NP & $11,20,31$ \\
\hline - & - & - & - & - & - & NP & 34 \\
\hline- & - & 12 & 14 & 3 & 6 & NP & 33 \\
\hline
\end{tabular}

\section{Parity / gravidity (n)}

Nulli Primi

Multi Reference

Duration of amenorrhea at

FMD measurement (weeks)

$\begin{array}{llllllll}\text { R } & \text { GHD } & \text { R } & \text { GHD } & \text { R } & \text { GHD } & & \\ - & - & 33 & 33 & - & - & \text { PP (3-6 months) } & 35 \\ 19 & 19 & - & - & - & - & \text { PP (3 months) } & 35 \\ - & - & 12 & 15 & 3 & 5 & \text { NP } & 34\end{array}$


Adequate description of participants' characteristics

Parity or gravidity

Health or comorbidities of participants

Clear reporting of weeks amenorrhea

Study Participation

Ethnicity

Height

Non-pregnant weight/BMl

Use of medication or supplements

Adequate description of participant recruitment

Adequate description of inclusion and exclusion criteria

Reasons for loss to follow-up/drop-out are provided

Study Attrition

Adequate description of participants lost to follow-up / differences between participants who completed and drop-outs

Method of measurement is adequately valid and reliable

Variable Measurements

The methods and setting are the same for all study participants and throughout follow up

Data Reporting

Time frame of measurements (gestational age) are reported as mean Study used a longitudinal study design

Study Design

Multiple (>2) longitudinal pregnant measurements during pregnancy of the variable

Baseline value was a pre-pregnant measurement of the variable

Score (\%)

Quality

Table 4 Quality assessment of included studies based on modified QUIPS criteria. The individual studies are defined as a high quality study ( $\mathrm{HQ})$, moderate quality study (MQ) or low quality study (LQ) 


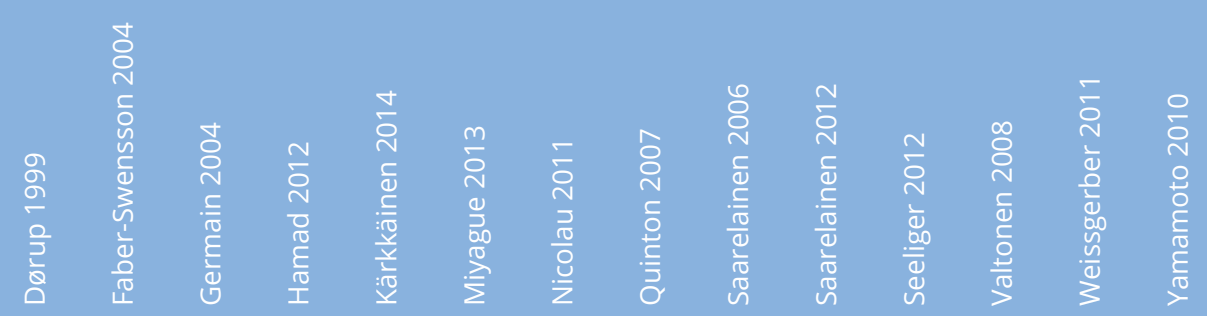

\begin{tabular}{|c|c|c|c|c|c|c|c|c|c|c|c|c|}
\hline+ & - & + & - & + & - & + & - & - & + & + & + & + \\
\hline+ & + & + & + & + & + & + & + & + & + & + & + & + \\
\hline
\end{tabular}

\begin{tabular}{|c|c|c|c|c|c|c|c|c|c|c|c|c|c|}
\hline- & - & - & - & - & - & - & - & - & - & - & - & - & - \\
\hline- & - & - & + & + & - & - & + & + & - & + & + & - & - \\
\hline+ & + & - & - & - & + & - & - & - & - & + & - & + & + \\
\hline+ & + & - & + & - & + & - & + & - & - & + & - & + & + \\
\hline$?$ & $?$ & + & $?$ & + & + & + & + & $?$ & + & + & $?$ & + & $?$ \\
\hline
\end{tabular}

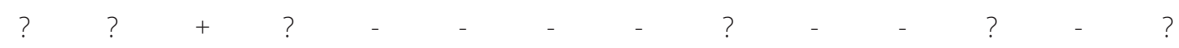

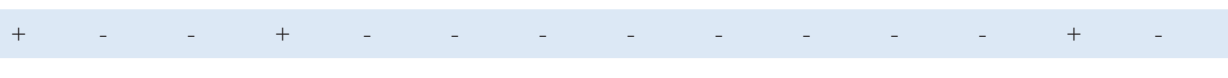

\begin{tabular}{|c|c|c|c|c|c|c|c|c|c|c|c|c|c|}
\hline+ & + & - & - & + & + & + & + & - & + & + & + & + & + \\
\hline- & + & + & + & - & + & - & + & - & + & + & - & - & - \\
\hline
\end{tabular}

\begin{tabular}{llllllllllllll}
47 & 53 & 35 & 53 & 41 & 65 & 29 & 53 & 24 & 47 & 65 & 35 & 59 & 41 \\
$M Q$ & $M Q$ & $M Q$ & $M Q$ & $M Q$ & $H Q$ & $L Q$ & $M Q$ & $L Q$ & $M Q$ & $H Q$ & $M Q$ & $M Q$ & $M Q$ \\
\hline
\end{tabular}


Domain

Appropriate Ultrasound Technology

Subject preparation

Baseline measurements

Vascular occlusion

Reactive hyperemia measurements

FMD analysis

\section{Items for consideration}

High resolution probe

Duplex mode

Angle steer and insonation angle correction

ECG gating

Intensity-weighted velocity calculations

Vitamin supplementation

Drug use

Tobacco use

Caffeine use

Menstrual Phase

Previous exercise/rested state

Fasted state

Adequate acclimatization

Repeated measurements

Baseline arterial diameter

Baseline blood velocity

Cuff type

Cuff position

Cuff duration

Temporal kinetics of arterial diameters and blood velocities

Calculation of shear rate

Edge detection software

Calculation of FMD

Normalization of FMD

score (\%)

Quality

Table 5 Quality assessment of FMD measurement of the included studies based on Harris criteria.

The individual studies are defined as moderate quality study (MQ) or low quality study (LQ). 


\begin{tabular}{|c|c|c|c|c|c|c|c|c|c|c|c|c|c|}
\hline $\begin{array}{l}\text { ㅇ } \\
\text { ㅇ } \\
\frac{\circ}{2} \\
\frac{1}{8}\end{array}$ & 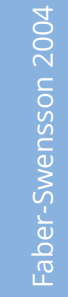 & 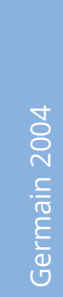 & $\begin{array}{l}\text { N } \\
\text { N } \\
\text { O } \\
\frac{\pi}{1} \\
\frac{1}{1}\end{array}$ & 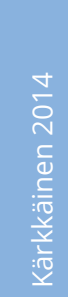 & 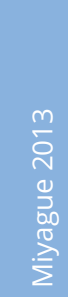 & 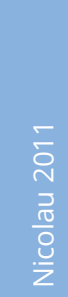 & 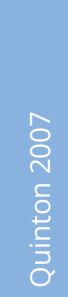 & 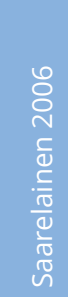 & 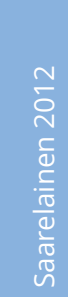 & $\begin{array}{l}\text { ㅁ } \\
\text { N } \\
\frac{1}{0} \\
\frac{.0}{\bar{d}} \\
\sim \\
\sim\end{array}$ & 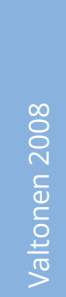 & $\begin{array}{l}\frac{\sigma}{0} \\
\text { v } \\
\frac{1}{d} \\
\frac{0}{1} \\
\frac{1}{d} \\
\text { D. } \\
\frac{n}{d} \\
3\end{array}$ & 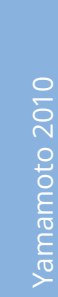 \\
\hline- & + & + & - & + & + & + & + & $?$ & $?$ & + & + & + & - \\
\hline$?$ & $?$ & $?$ & $?$ & $?$ & $?$ & $?$ & $?$ & $?$ & $?$ & + & $?$ & + & $?$ \\
\hline - & $?$ & $?$ & $?$ & $?$ & $?$ & $?$ & + & $?$ & $?$ & - & ? & - & $?$ \\
\hline+ & - & - & $?$ & + & + & + & + & + & + & + & + & $?$ & $?$ \\
\hline$?$ & $?$ & $?$ & $?$ & $?$ & $?$ & $?$ & $?$ & $?$ & $?$ & $?$ & $?$ & $?$ & $?$ \\
\hline$?$ & $?$ & $?$ & $?$ & $?$ & $?$ & $?$ & $?$ & $?$ & $?$ & - & $?$ & $?$ & $?$ \\
\hline- & + & $?$ & $?$ & $?$ & - & $?$ & $?$ & $?$ & $?$ & + & $?$ & - & - \\
\hline+ & + & + & + & - & + & + & + & - & + & + & - & + & + \\
\hline$?$ & $?$ & + & $?$ & $?$ & + & - & + & $?$ & $?$ & - & $?$ & + & $?$ \\
\hline$?$ & - & - & $?$ & $?$ & $?$ & + & + & $?$ & $?$ & + & - & - & $?$ \\
\hline$?$ & $?$ & $?$ & $?$ & $?$ & $?$ & $?$ & $?$ & $?$ & $?$ & $?$ & $?$ & + & $?$ \\
\hline$?$ & $?$ & + & $?$ & + & + & + & + & $?$ & - & + & $?$ & - & $?$ \\
\hline- & - & - & $?$ & $?$ & - & - & - & $?$ & $?$ & - & $?$ & - & $?$ \\
\hline- & $?$ & - & $?$ & $?$ & $?$ & $?$ & $?$ & $?$ & - & $?$ & $?$ & $?$ & $?$ \\
\hline - & - & - & + & $?$ & - & - & - & $?$ & $?$ & - & $?$ & - & $?$ \\
\hline+ & $?$ & $?$ & $?$ & $?$ & $?$ & $?$ & $?$ & $?$ & $?$ & $?$ & $?$ & $?$ & $?$ \\
\hline$?$ & $?$ & - & $?$ & $?$ & - & $?$ & - & $?$ & - & $?$ & $?$ & - & $?$ \\
\hline+ & + & + & + & + & + & + & + & + & + & + & + & + & - \\
\hline- & - & + & + & - & + & + & + & - & - & + & - & + & $?$ \\
\hline- & - & - & - & - & $?$ & - & - & - & - & $?$ & - & + & - \\
\hline$?$ & $?$ & - & $?$ & $?$ & $?$ & $?$ & $?$ & $?$ & $?$ & $?$ & $?$ & - & $?$ \\
\hline- & $?$ & - & + & $?$ & $?$ & $?$ & $?$ & $?$ & $?$ & $?$ & $?$ & + & $?$ \\
\hline$?$ & - & + & + & + & + & - & + & + & $?$ & + & + & $?$ & - \\
\hline$?$ & $?$ & - & $?$ & $?$ & $?$ & $?$ & $?$ & $?$ & $?$ & $?$ & $?$ & + & $?$ \\
\hline 17 & 17 & 29 & 25 & 21 & 33 & 29 & 42 & 13 & 13 & 42 & 17 & 42 & 4 \\
\hline LQ & LQ & LQ & LQ & LQ & $\mathrm{MQ}$ & LQ & $\mathrm{MQ}$ & LQ & LQ & $\mathrm{MQ}$ & LQ & $\mathrm{MQ}$ & LQ \\
\hline
\end{tabular}


d

4

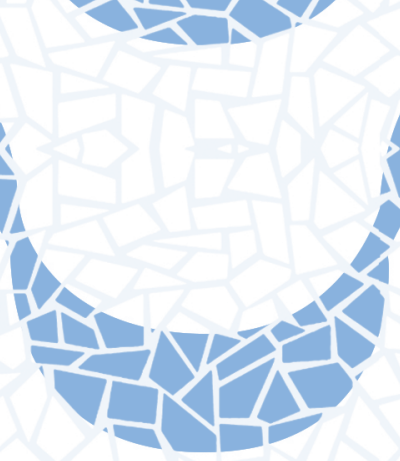

40.2
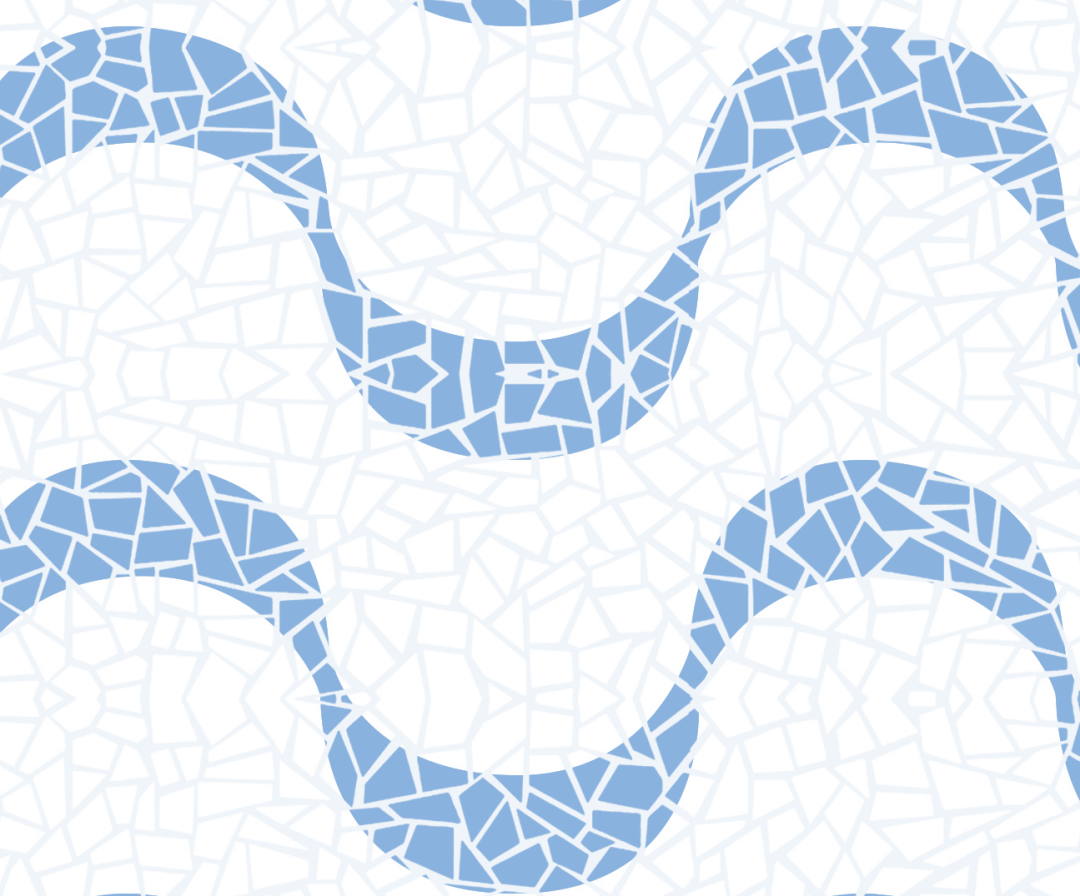

$4 \leq 1$

पर्थ

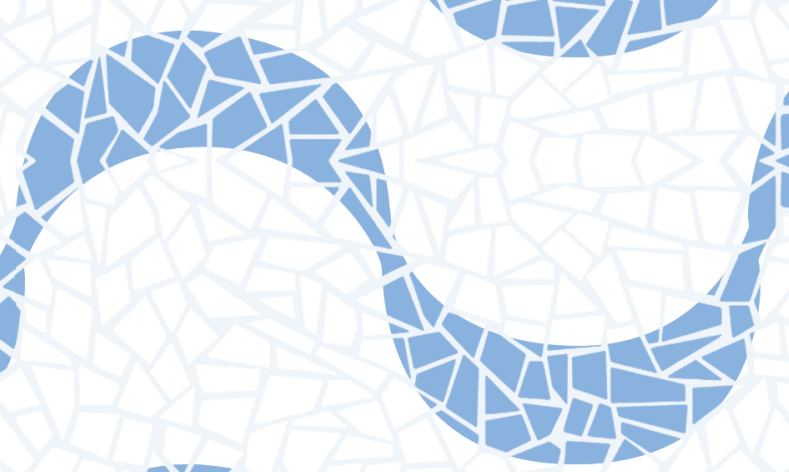

करो
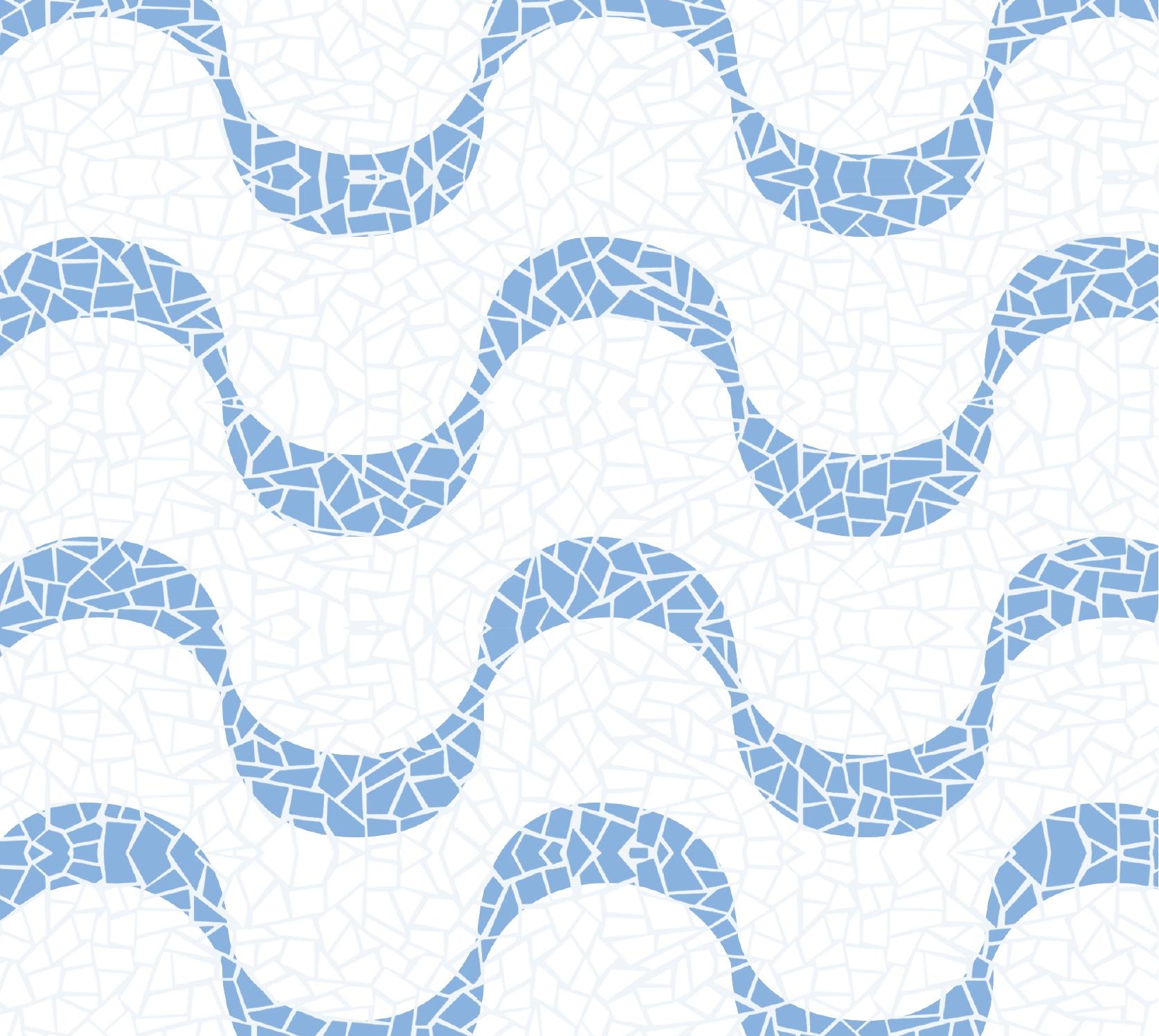


\title{
CHAPTER 3
}

\section{Kidney function during pregnancy: a systematic review and meta-analysis}

\author{
Veronica A. Lopes van Balen \\ Tessa A.G. van Gansewinkel \\ Sander de Haas \\ Julia J. Spaan \\ Chahinda Ghossein-Doha \\ Sander M.J. van Kuijk \\ Joris van Drongelen \\ Tom Cornelis \\ Marc E.A. Spaanderman
}

Accepted for publication in Ultrasound Obstetrics and Gynecology 


\section{ABSTRACT}

OBJECTIVES The purpose of this meta-analysis is to systematically review current literature on kidney function changes during pregnancy, to estimate the extent of adaptation over the course of both healthy physiological and hypertensive complicated singleton pregnancies and to determine healthy pregnancy reference values.

METHODS A meta-analysis was performed following a systematic review of current literature on kidney function during uncomplicated and complicated pregnancies. PubMed (NCBI) and Embase (Ovid) electronic databases were used for the literature search, which was performed from inception to July 2017. Studies were required to report a non-pregnant reference value of kidney function (either non-pregnant control group, prepregnancy or postpartum measurement) and a pregnancy measurement during a predetermined and reported gestational age. Pooled mean differences between pregnancy measurements and reference value were calculated for predefined intervals of gestational age using a random-effects model described by DerSimonian and Laird.

RESULTS Twenty-nine studies were included with publication dates ranging from 1958 to 2016. As early as the first trimester, glomerular filtration rate (GFR) increases, up until 40 - 50\% during physiologic pregnancy when compared to non-pregnant conditions. Inulin clearance was highest at 36 - 41 weeks with a $55.6 \%$ increase $(\Delta 53.7$ $\mathrm{ml} / \mathrm{min} \mathrm{Cl} 44.7$ - 62.6), and creatinine clearance at $15-21$ weeks with a $37.6 \%$ increase $(\Delta 36.6 \mathrm{ml} / \mathrm{min} \mathrm{Cl} 26.2-46.9)$. Serum creatinine values decrease was most prominent at $15-21$ weeks with a $23.2 \%$ decrease $(\Delta-0.19 \mathrm{mg} / \mathrm{dl}(\mathrm{Cl}-0.23--0.15)$ compared to non-pregnant conditions. The meta-regression analysis, containing eight studies on complicated pregnancies, showed a significant difference in for all kidney function parameters when comparing uncomplicated to complicated gestation.

CONCLUSIONS In healthy pregnancy GFR accelerates as early as the first trimester and remains to function at a higher rate throughout gestation. In contrast, kidney function is decreased in hypertensive gestation. Although the upper limit of the reference curve changes throughout gestation, from a clinical perspective, a serum creatinine above $0.75 \mathrm{mg} / \mathrm{dl}$ ( $66 \mathrm{umol} / \mathrm{L}$ ) should be considered abnormal during pregnancy. 


\section{INTRODUCTION}

A decreased vascular resistance is thought to induce adaptations that occur during normal, physiologic pregnancy and are sustained by an increased nitric oxide production ${ }^{1,2}$. The decrease in resistance initiates a chain of events that results in a rise of cardiac output and abets the expansion of plasma volume by stimulating renal sodium and water retention, and sodium appetite and thirst ${ }^{3}$. Both increased renal blood flow and decreased oncotic pressure due to plasma volume expansion contribute to higher glomerular filtration rate $(\mathrm{GFR})^{4,5}$.

In humans, this increase in GFR is reported as early as the first weeks after conception and is usually sustained until the end of gestation, but data reported show considerable individual variation ${ }^{6}$. Pregnancies complicated by hypertensive disease show decreased kidney function. Despite this knowledge reference curves of kidney function in pregnancy are lacking in literature. ${ }^{7,8}$. This meta-analysis therefore aims to systematically review and quantify current literature on kidney function during pregnancy, to estimate the extent of adaptation over the course of both physiological and hypertensive complicated singleton pregnancies. Kidney function is represented by GFR and serum creatinine. Finally, we aim to construct GFR and serum creatinine levels references curves in healthy pregnancy, with marked $5^{\text {th }}, 50^{\text {th }}$ and $95^{\text {th }}$ percentile. 


\section{METHODS}

\section{LITERATURE SEARCH}

Studies evaluating kidney function during physiologic uncomplicated and hypertensive complicated pregnancies were collected using PubMed (NCBI) and Embase (Ovid). We searched publications in these electronic databases from inception until July 2017. The search strategy focused on pregnancy, both uncomplicated and complicated, and kidney function, as detailed in Table 1. Complicated pregnancy can be subdivided in pregnancy induced hypertension (PIH), foetal growth restriction (FGR), preeclampsia (PE) and gestational diabetes (GDM). All components consisted of free search terms in titles and abstracts and $\mathrm{MeSH}$ - and Emtree terms for respectively Pubmed and Embase databases. Reference lists of all included studies were reviewed for additional studies.

\section{SELECTION OF STUDIES}

Studies were qualified for inclusion following a two-phased selection process as illustrated in Figure 1. First, all obtained articles were screened independently for eligibility by two investigators (VLVB, TVG) based on title and abstract only. Discrepancies were resolved by mutual agreement. Subsequently, articles that passed the first selection phase were read completely and screened for inclusion based on the inclusion and exclusion criteria by the same investigators. Studies were eligible for inclusion if they reported original data, including mean GFR, estimated GFR (eGFR) or serum creatinine during human singleton pregnancies with either standard deviation (SD), standard error (SE), or 95\% confidence interval $(95 \%$ CI). We assumed that values were normally distributed. Studies had to include a reference measure of either pre-pregnant, non-pregnant or postpartum (> 6 weeks) controls. Pre-pregnant and postpartum measurements subjects were also measured during pregnancy. This was relevant in order to be able to calculate differences between pregnant and reference measurements within each study. Additionally, no restrictions were set on age, weight, height, ethnicity or parity. Exclusion criteria were as follows: studies assessing women with comorbidities (such as pre-existing cardiovascular history, diabetes mellitus, kidney disease and immunological disease), studies reporting data in other languages than English, Dutch, Spanish, Italian, Portuguese, French or German. Case reports and reviews were also excluded. Measurements reported after interventions were not used but baseline measurements of an intervention study were ${ }^{9-12}$.

\section{DATA EXTRACTION}

Data regarding study characteristics, anthropometric measures and methods used to measure (e)GFR and serum creatinine were extracted from the selected studies. Regarding study characteristics, the following were obtained: authors, year of publication, study design, sample size, population description, in- and exclusion criteria and key conclusions. 
Additionally, extracted subject characteristics consisted of age, non-pregnant weight and body mass index (BMI), height, parity, gravidity, body surface area, gestational age at time of (e)GFR or serum creatinine measurement, birthweight of offspring, and gestational age at delivery. For women with a gestational hypertensive disease gestational age, at onset of the hypertensive disorder, was documented. Effect measures include (e)GFR and serum creatinine as primary outcomes, heart rate (HR), systolic blood pressure (SBP), diastolic blood pressure (DBP) and mean arterial pressure (MAP) as secondary outcomes. We defined GFR as kidney function measured by either inulin or creatinine clearance (in ml/ min), and eGFR as kidney function measured by any formula based on serum creatinine (in $\mathrm{ml} / \mathrm{min} / 1.73 \mathrm{~m}^{2} \mathrm{or} \mathrm{ml} / \mathrm{min}$ ). Data on GFR measurement protocol, eGFR formulas and serum creatinine analysis were also extracted. If a study contained more than one measurement during our predefined intervals the mean value was calculated ${ }^{13,14}$. Requests were made to retrieve additional information from the authors of the included articles if data were unclear or incomplete.

\section{QUALITY ASSESSMENT}

The quality and risk of bias of included studies were assessed independently by two investigators according to a modified set of items as reported in the Quality in Prognosis Study (QUIPS) tool ${ }^{15}$. This modification was made to suit the purposes of this review. A plus or minus or interrogation mark (that counts as a minus and was used when follow-up was not applicable) was allocated per study for risk of bias on five domains: study participation, study attrition, variable measurement, data reporting, and study design. All domains were deemed of equal importance, and thus weighed accordingly in the total score. Studies with a positive score $\geq 60 \%$ were defined as high quality (HQ), those scoring $\geq 30 \%$ and $<$ $60 \%$ as moderate quality (MQ) and those scoring < $30 \%$ as low quality (LQ).

\section{DATA AND STATISTICAL ANALYSIS}

(e)GFR and serum creatinine were categorized into five different intervals of gestational age ( $<14,15$ - 21, 22 - 28, 29 - 35, and 36 - 41 weeks). These gestational age intervals were adapted from Abudu et el. ${ }^{16}$. SD was obtained from SE or 95\% Cl and calculated for combined groups according to the Cochrane handbook for systematic review of interventions whenever necessary ${ }^{17}$. (e)GFR and serum creatinine change were calculated separately for these predefined intervals using a random-effects model as described by DerSimonian and Laird ${ }^{18}$. The random-effects model allows for inter-study variation and was chosen by design since observational data of different pregnant populations were used. The primary outcome of each study was the mean difference in (e)GFR and serum creatinine between pregnancy and reference value, reported with a 95\% Cl. 
Reference values in healthy pregnancy were constructed by plotting the mean value for each individual study of the reference measurement (non-pregnant), the mean value at a certain gestational age and the value at a certain time postpartum. The $5^{\text {th }}$ and $95^{\text {th }}$ percentile were calculated from intra-study variance as 95\% prediction intervals. Each point estimate was indexed as a value from a LQ, MQ or HQ study as stipulated by study quality assessment. The meta-analyses and meta-regression analyses were performed in $\mathrm{R}$ version 3.2.3 using the meta package V4.8-4 ${ }^{19,20}$. Forest plots present the results of the meta-analysis. The ratio between total heterogeneity and total variability (I-squared statistic $\left.\left(I^{2}\right)\right)$ was computed as a measure of heterogeneity. $I^{2}$ can distinguish true heterogeneity from sampling variance and is expressed as a percentage ${ }^{21}$. Sources of heterogeneity were investigated by meta-regression analyses using a mixed-effects model. Egger's regression test for funnel plot asymmetry was performed to test for the presence of publication bias ${ }^{22}$. 


\section{RESULTS}

\section{STUDY AND DATA SELECTION}

The literature search resulted in 10160 articles after duplicate removal from both Pubmed and Embase electronic databases, as illustrated in Figure 1. Subsequently, screening records based on title and abstract yielded 176 studies eligible for full-article analysis of which a large number of studies were later precluded due to unobtainable data $(n=74)$. We excluded 147 full text articles for several reasons, which are further detailed in Figure 1. Requests made to retrieve additional information did not result in additional information.

Ultimately, 29 studies were included incorporating 20 preconceptional, 376 non-pregnant, 1037 healthy pregnant, 204 hypertensive complicated pregnancy and 246 postpartum kidney function values. No additional articles were found in the reference sections and none were excluded based on language. Three studies, two of which are included in this review and analysis, contained eGFR values ${ }^{23-25}$; all other studies reported GFR either measured by inulin or creatinine clearance. We were therefore unable to perform a metaanalysis on eGFR. Four studies contained values of inulin clearance expressed as m// $\mathrm{min} / 1.73 \mathrm{~m}^{2}$, which we were unable to process in this meta-analysis without measures of body surface area ${ }^{26-28}$.

Two studies reported results from repeated measures during the predefined interval and we opted to calculate a mean and SD for these studies, ignoring potential clustering 13,29. In a study containing 398 healthy pregnant participants a total of six women developed preeclampsia in late pregnancy, these women were not excluded from statistical analysis in this study, as well as from our meta-analysis due to the marginal suspected effect it might have had on the results ${ }^{30}$. Because our meta-analysis showed a significant difference between GFR measured by inulin and GFR measured by creatinine clearance $(p<0.001)$, we opted to analyse both separately. 

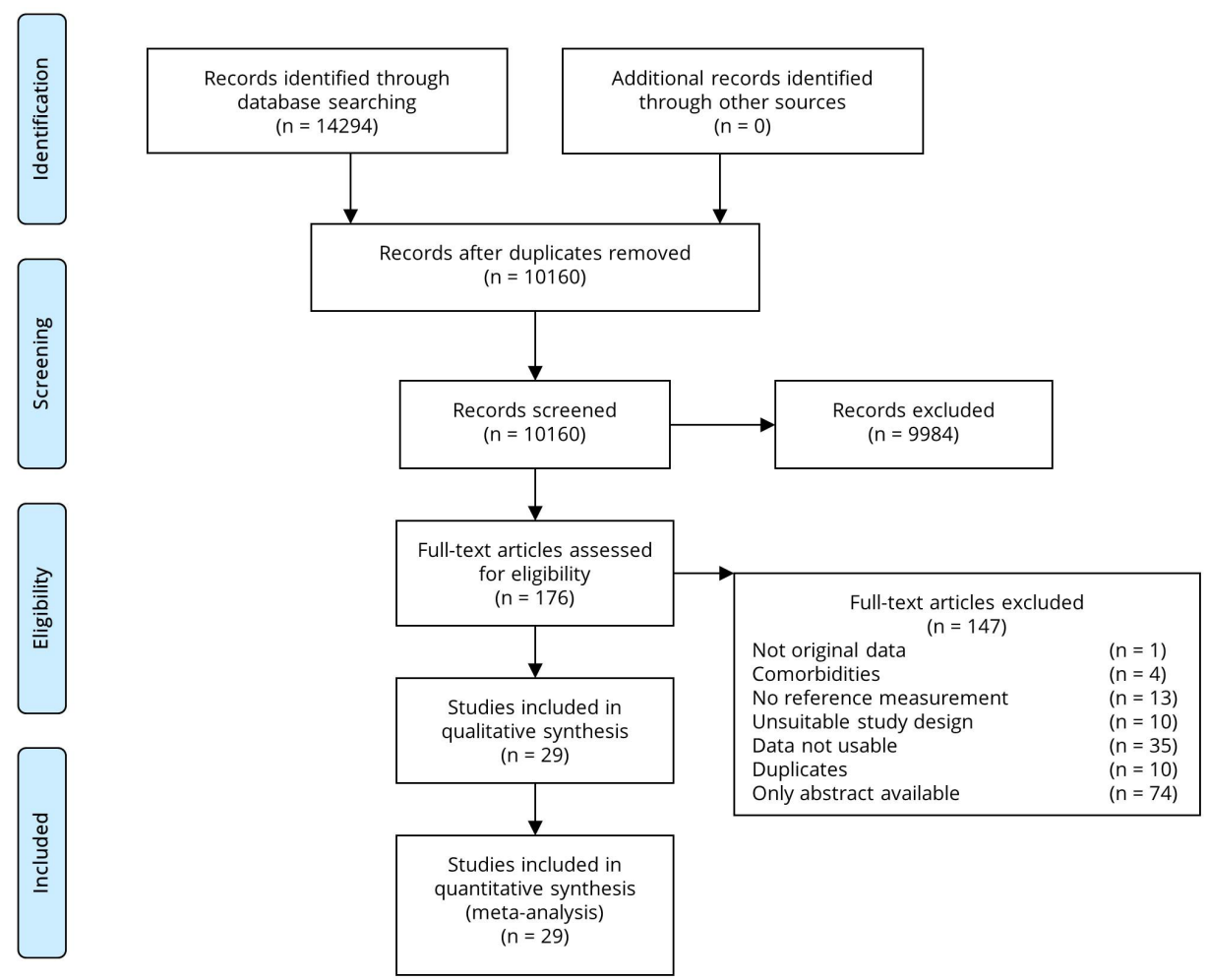

Figure 1 Flow chart for selection, inclusion and exclusion of studies.

\section{DATA EXTRACTION}

Study characteristics and anthropometric measures are illustrated in Tables 2 and 3, for uncomplicated pregnancies and pregnancies complicated by gestational hypertensive diseases, respectively. For most studies the overall characteristics are detailed. Included studies contained women with hypertensive complicated pregnancies, namely gestational hypertension ${ }^{31}$ and preeclampsia ${ }^{31-38}$. We found no studies containing women with gestational diabetes. Participant's anthropometric data were infrequently and incompletely reported by the authors. It was therefore impossible to analyse the contribution of these variables to the heterogeneity observed. Tables 4 through 6 illustrate for each study the measurement methods used for GFR measured by inulin, GFR measured by creatinine clearance and serum creatinine. 


\section{QUALITY ASSESSMENT}

Quality assessment of the included studies is depicted in Table 7a and 7b. Most studies were of $\mathrm{MQ}(n=15)$, whilst the remaining were subdivided in $\mathrm{HQ}(n=9)$ and $\mathrm{LQ}(n=5)$. Quality scores varied between 12\% and 82\% (median 53\%; IQR 41 - 65\%). Each study scored best on 'the methods and setting are the same for all study participants and throughout follow-up', while the least scored items were: 'baseline value was a prepregnant measurement of the variable' $(n=2)$, 'multiple $(>2)$ longitudinal measurements during pregnancy of the variable' $(n=6)$ and 'adequate description of participant's ethnicity characteristics' $(n=7)$.

\section{GLOMERULAR FILTRATION RATE - INULIN CLEARANCE (GFR - IC)}

A forest plot illustrating GFR-IC course during uncomplicated pregnancies compared to reference conditions is depicted in Figure 2. GFR-IC, when compared to reference values, is increased during uncomplicated pregnancy at all gestational age intervals $(<14,15-21$, 22 - 28, 29 - 35 and 36 - 41 weeks' gestation), with $40.7 \mathrm{ml} / \mathrm{min}(14.1$ - $67.3 \mathrm{ml} / \mathrm{min})$, relative $37.7 \%$ (13.1 - 62.4\%), $47.8 \mathrm{ml} / \mathrm{min}$ (35.1 - $60.6 \mathrm{ml} / \mathrm{min})$, relative $46.6 \%$ (34.2 - 59.1\%), 44.8 $\mathrm{ml} / \mathrm{min}(29.2$ - $60.4 \mathrm{ml} / \mathrm{min})$, relative $40.2 \%(26.2-54.1 \%), 39.6 \mathrm{ml} / \mathrm{min}(27.1-52.1 \mathrm{ml} /$ $\mathrm{min})$, relative $35.6 \%$ (24.4 - 46.8\%), and $53.7 \mathrm{ml} / \mathrm{min}(44.7-62.6 \mathrm{ml} / \mathrm{min})$, relative $55.6 \%$ (46.3 - 64.9\%), respectively. The meta-regression analysis showed no significant effect of gestational age on the course of GFR-IC measurement ( $p=0.789$ ).

In terms of study-level covariates, to interpret heterogeneity, a significant statistical association between GFR-IC and reference measurement (postpartum vs. non-pregnant, $p=0.001$, there were no pre-pregnancy measurements) and GFR-IC and different study qualities (MQ vs. HQ, p = 0.036, there is only one LQ study and its effect could therefore not be calculated) was found. Eggers's funnel plot asymmetry could not be calculated at < 14 weeks due to a lack of studies, but was not statistically significant at any other interval $(0.254 \leq p \leq 0.721)$.

Two included studies report data on GFR-IC during complicated pregnancies (Figure 3) between 29 - 35 weeks and 36 - 41 weeks respectively. In contrast to uncomplicated pregnancies, a significant decrease of $-62.4 \mathrm{ml} / \mathrm{min}(-75.9--48.9 \mathrm{ml} / \mathrm{min})$, relative $-51.2 \%$ $(-62.3--40.2 \%)$ in GFR-IC was seen at $29-35$ weeks, and no effect was seen at $36-41$ weeks (-3.0 $\mathrm{ml} / \mathrm{min}(-8.7-2.7 \mathrm{ml} / \mathrm{min})$, relative $-3.3 \%(-9.5-3.0 \%))$, compared to reference values. These results differed significantly from uncomplicated pregnancies $(p<0.001)$. Reference values for GFR-IC throughout physiological, uncomplicated pregnancy are presented in Figure 4, where we observed an overall increase during pregnancy and a slight decrease after 28 weeks of gestational age. 


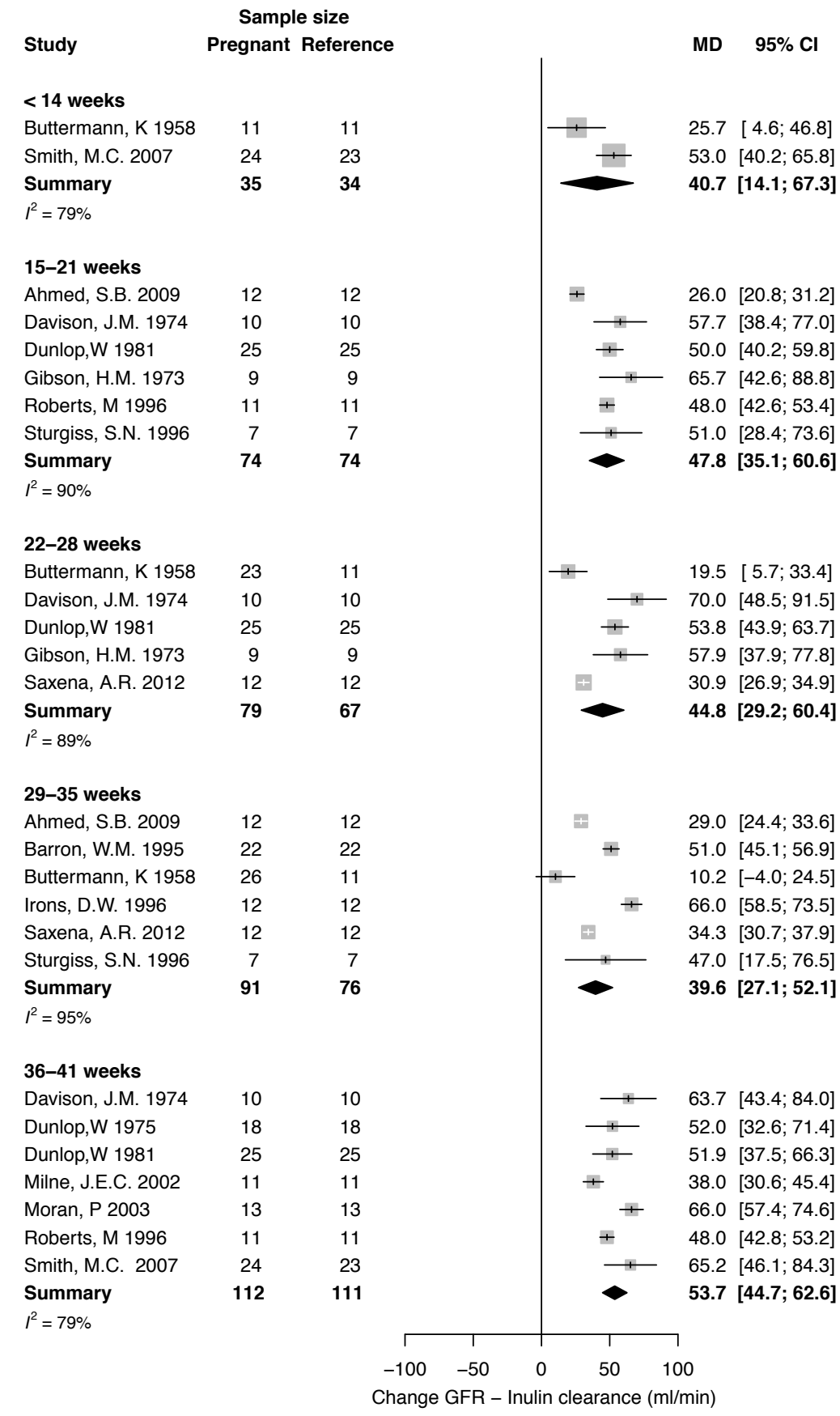

Figure 2 Forest plot of glomerular filtration rate (GFR), measured with inulin in $\mathrm{ml} / \mathrm{min}$, during physiologic pregnancies at < 14 weeks, 15 - 21 weeks, 22 - 28 weeks, 29 - 35 weeks, and 36 - 41 weeks' gestation compared to the reference value. GFR is reported as mean difference (MD) between pregnant and reference values, with 95\% Cl. 


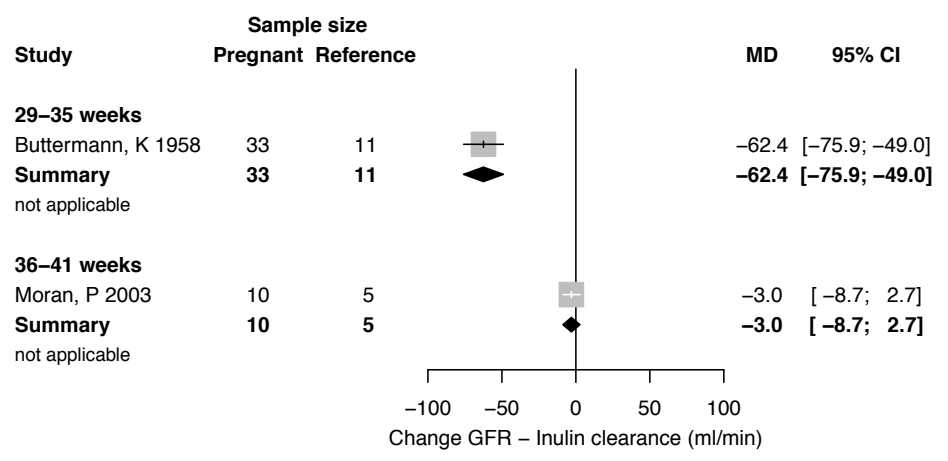

Figure 3 Forest plot of glomerular filtration rate (GFR), measured by inulin in $\mathrm{ml} / \mathrm{min}$, during hypertensive complicated pregnancies at 29 - 35 and 36 - 41 weeks' gestation compared to the reference value. GFR is reported as mean difference (MD) between pregnant and reference values, with $95 \% \mathrm{Cl}$.

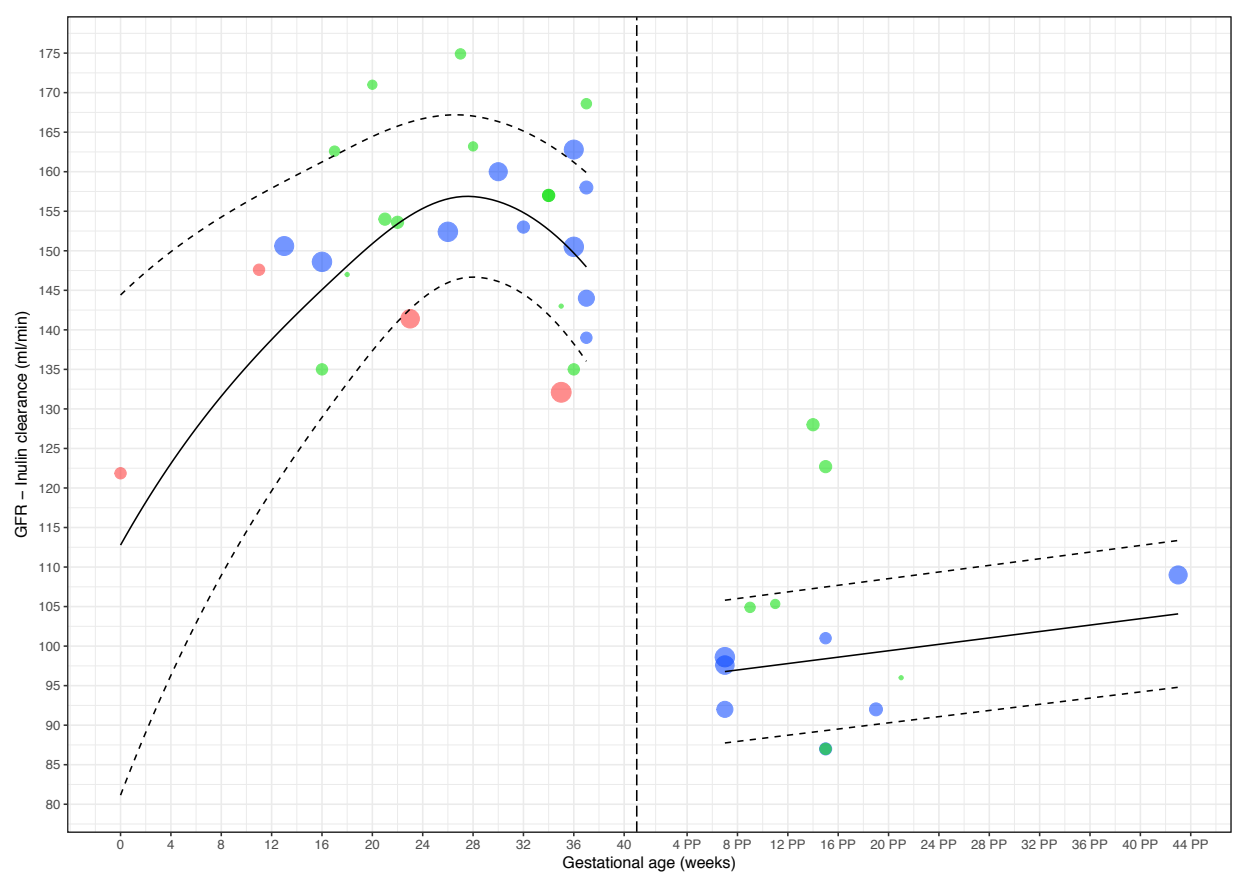

Figure 4 Increase in glomerular filtration rate (GFR), measured by inulin in $\mathrm{ml} / \mathrm{min}$, during physiologic pregnancy. The size of the circle indicates the sample size of the point estimate. The colour of the circle refers to the quality assigned to the study: red $=L Q$, green $=M Q$, blue $=H Q$. Curve fit is weighted by the inverse variance and plotted with $5^{\text {th }}$ and $95^{\text {th }}$ percentiles. The $50^{\text {th }}$ percentile is represented by a solid line. 
GLOMERULAR FILTRATION RATE - CREATININE CLEARANCE (GFR - CC)

A forest plot of GFR-CC in uncomplicated pregnancies compared to reference value is illustrated in Figure 5. When compared with the reference conditions, GFR-CC increased significantly throughout uncomplicated pregnancy.

GFR-CC increased progressively at $<14$ and 15 - 21 weeks of gestation with $22.0 \mathrm{ml} / \mathrm{min}$ (9.9 - $43.0 \mathrm{ml} / \mathrm{min})$, relative $23.7 \%(10.7$ - 36.7\%), and $36.6 \mathrm{ml} / \mathrm{min}(26.2-46.9 \mathrm{ml} / \mathrm{min})$, relative $37.6 \%$ (26.9 - 48.2\%), respectively. From 22 - 28 weeks of gestation onwards GFR was still increased by $28.7 \mathrm{ml} / \mathrm{min}$ (16.8 - $40.6 \mathrm{ml} / \mathrm{min})$, relative $26.9 \%$ (15.7 - 38.0\%), and $16.0 \mathrm{ml} / \mathrm{min}(9.1-22.9 \mathrm{ml} / \mathrm{min})$, relative $15.1 \%$ (8.6 - 21.7\%), at 29 - 35 weeks, but to a lesser extent than in previous intervals. There was no increase at 36 - 41 weeks of gestation (-8.8 $\mathrm{ml} / \mathrm{min}$ (-54.4 - $36.8 \mathrm{ml} / \mathrm{min})$, relative $-8.0 \%$ (-49.6 - 33.5\%).

The meta-regression analysis objectified a significant difference in GFR-CC and gestational age ( $p=0.007)$, however, in contrast to GFR-IC no significant effect was seen between different reference measurements (pre-pregnant vs. non-pregnant $p=0.227$, postpartum vs. non-pregnant $p=0.811)$. There was an effect of $M Q$ vs. HQ studies ( $p=0.010$ ) but no effect of LQ vs. HQ studies ( $p=0.07$ ) on the course of GFR-CC. Egger's test for funnel plot asymmetry found no indicatives for publication bias at any gestational age interval $(0.344 \leq \mathrm{p} \leq 0.697)$.

Six included studies reported data on GFR-CC in hypertensive complicated pregnancies (Figure 5). GFR-CC showed a significant decrease of $-14.7 \mathrm{ml} / \mathrm{min}(-22.1--7.3 \mathrm{ml} /$ min), relative $-14.1(-21.2--7.1 \%)$, at 29 - 35 weeks of gestation when compared to reference measurements, which was also significantly different when compared to healthy pregnancies at the same interval $(p=0.008)$. Figure 7 portrays reference values for GFR-CC throughout physiological, uncomplicated pregnancies. There is an increase in GFR-CC until around 16 weeks and a decrease thereafter. After 24 weeks of pregnancy the heterogeneity between studies increased as can be seen by the amount of studies outside the reference curves, estimated using aggregated data. 


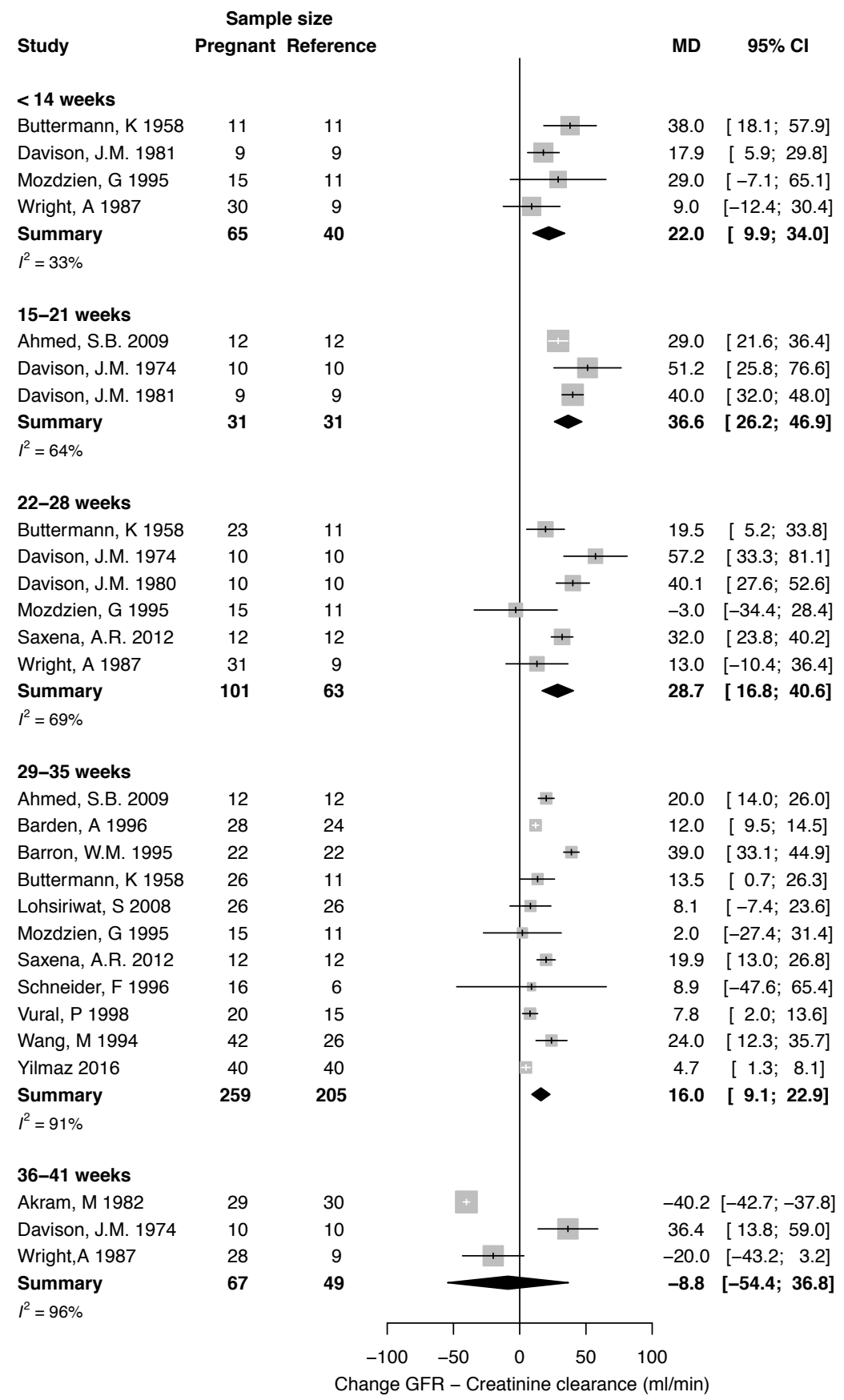

Figure 5 Forest plot of glomerular filtration rate (GFR), measured by creatinine clearance in $\mathrm{ml} /$ min, during physiologic, uncomplicated, pregnancies at $<14$ weeks, 15 - 21 weeks, 22 - 28 weeks, 29 - 35 weeks, and 36-41 weeks' gestation compared to the reference value. GFR is reported as mean difference (MD) between pregnant and reference values, with 95\% $\mathrm{Cl}$. 


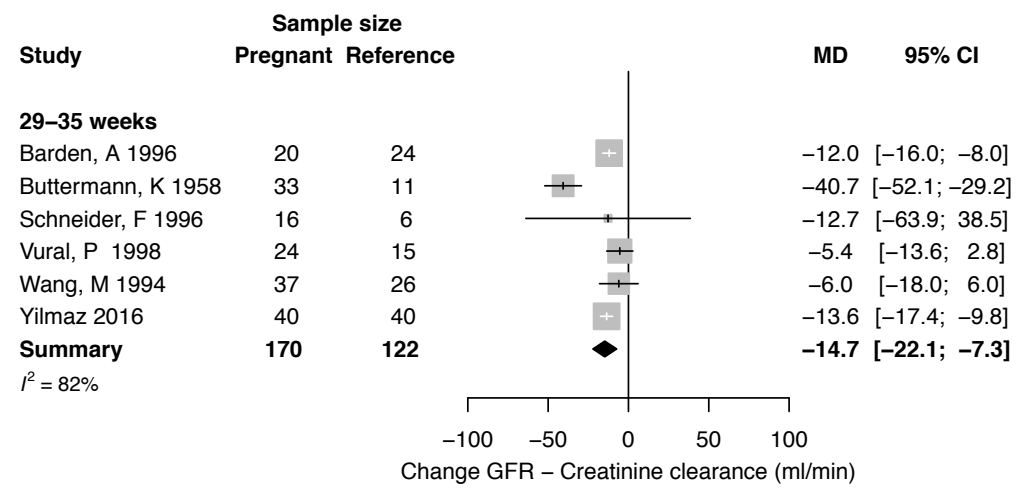

Figure 6 Forest plot of glomerular filtration rate (GFR), measured by creatinine clearance in $\mathrm{ml} /$ min, during hypertensive complicated pregnancies at 29 - 35 weeks' gestation compared to the reference value. GFR is reported as mean difference (MD) between pregnant and reference values, with $95 \% \mathrm{Cl}$.

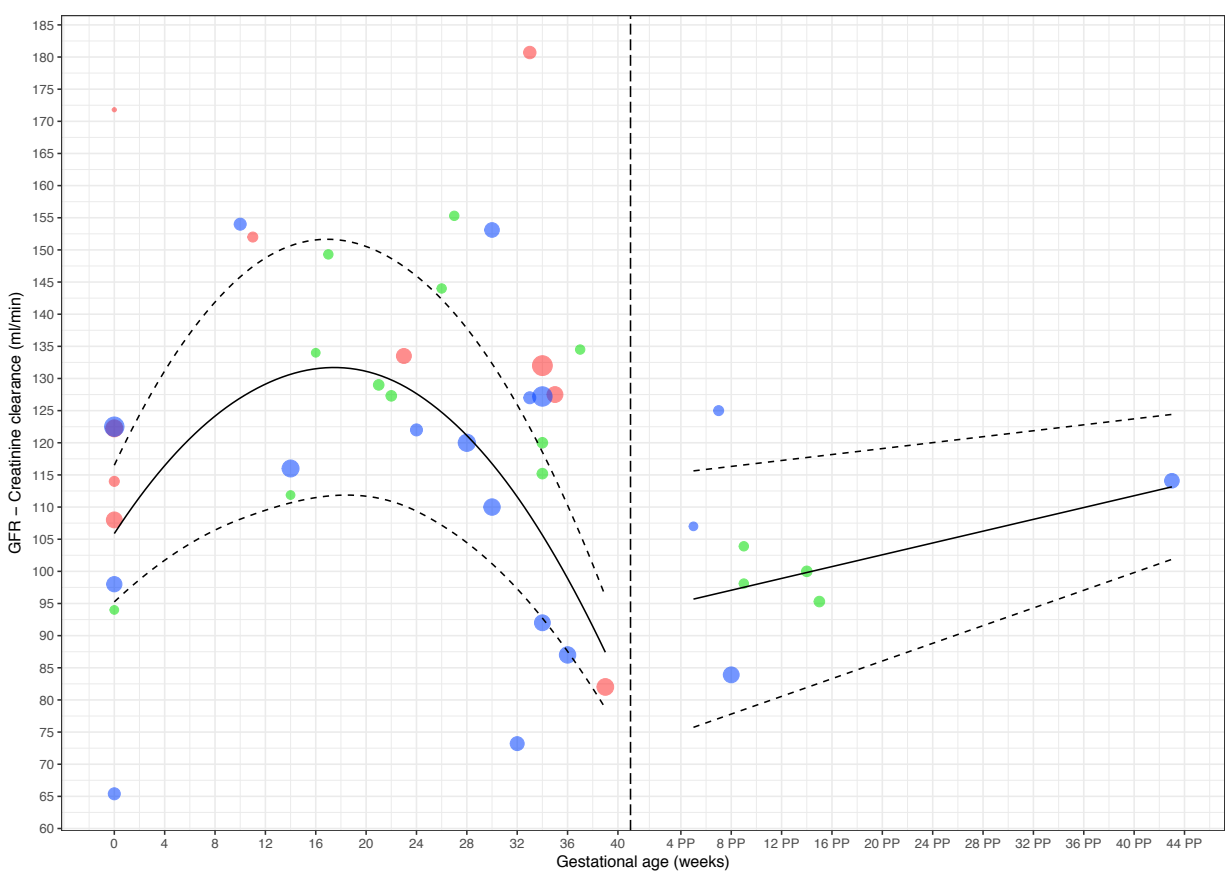

Figure 7 Increase in glomerular filtration rate (GFR), measured by creatinine clearance in $\mathrm{ml} / \mathrm{min}$, during physiologic pregnancy. The circle size indicates the sample size of the point estimate. The colour of the circle refers to the quality assigned to the study: red $=L Q$, green $=M Q$, blue $=H Q$. Curve fit is weighted by the inverse variance and plotted with $5^{\text {th }}$ and $95^{\text {th }}$ percentiles. The $50^{\text {th }}$ percentile is represented by a solid line. 
SERUM CREATININE

A forest plots of serum creatinine in uncomplicated pregnancies compared to the reference value is illustrated in Figure 8. Throughout uncomplicated pregnancy serum creatinine is decreased in comparison to non-pregnant reference conditions. At $<14$ weeks' gestation serum creatinine is seen at a slight decrement of $-0.13 \mathrm{mg} / \mathrm{dl}(-0.16$ $-0.11 \mathrm{mg} / \mathrm{dl})$, relative $-16.5(-20.4--14.0 \%)$, and it decreased further at $15-21$ weeks to $-0.19 \mathrm{mg} / \mathrm{dl}(-0.23--0.15 \mathrm{mg} / \mathrm{dl})$, relative $-23.2(-28.1--18.3 \%)$, only to reach a plateau at 22 - 29 weeks at $-0.18 \mathrm{mg} / \mathrm{dl}(-0.22--0.15 \mathrm{mg} / \mathrm{dl})$, relative $-22.6(-27.7--18.9 \%)$. At 29 - 35 weeks serum creatinine reaches the least difference between reference values of $-0.12 \mathrm{mg} / \mathrm{dl}(-0.15--0.10 \mathrm{mg} / \mathrm{dl})$, relative $-18.5(-15.5--13.0 \%)$, up to $-0.14 \mathrm{mg} / \mathrm{dl}$ $(-0.27--0.02 \mathrm{mg} / \mathrm{dl})$, relative $-17.7(-34.2--2.5 \%)$ at the end of pregnancy.

The meta-regression analysis showed that there was neither an effect of gestational age on the course of serum creatinine ( $p=0.687$ ), nor an effect of the reference measurements (pre-pregnant vs. non-pregnant, $p=0.553$ and postpartum vs. non-pregnant, $p=0.641$ ) or study quality (LQ vs. HQ p = 0.578 and MQ vs. HQ p = 0.157). Egger's regression test for funnel plot asymmetry was not statistically significant $(0.108<p<0.919)$. Studies with data on serum creatinine in hypertensive complicated pregnancies (Figure 9) reported data between 29-35 and 36-41 weeks' gestation.

In contrast to uncomplicated pregnancies, there was no change in serum creatinine in women with complicated pregnancies compared to non-pregnant values: $0.15 \mathrm{mg} /$ $\mathrm{dl}(-0.03-0.32)$, relative $21.2(-4.2-45.2 \%)$ at $29-35$ weeks and $-0.01 \mathrm{mg} / \mathrm{dl}(-0.16-$ $0.15 \mathrm{mg} / \mathrm{dl})$, relative $-1.4(-23.2-21.7 \%)$ at $36-41$ weeks. These values were however significantly different from uncomplicated pregnancy $(p=0.009)$. Reference values for serum creatinine, throughout physiological, uncomplicated pregnancy are depicted in Figure 10 and show a decrease during pregnancy until around 24 weeks after which it increases almost back to non-pregnant values. The upper range of the reference curve changes throughout gestation but a cut-off can be set at $0.75 \mathrm{mg} / \mathrm{dl}$ ( $66 \mathrm{umol} / \mathrm{L}$ ) based on the upper limit at the moment where serum creatinine is at its highest during pregnancy. In supplemental files all forest plots and reference values are also available in umol/L. 


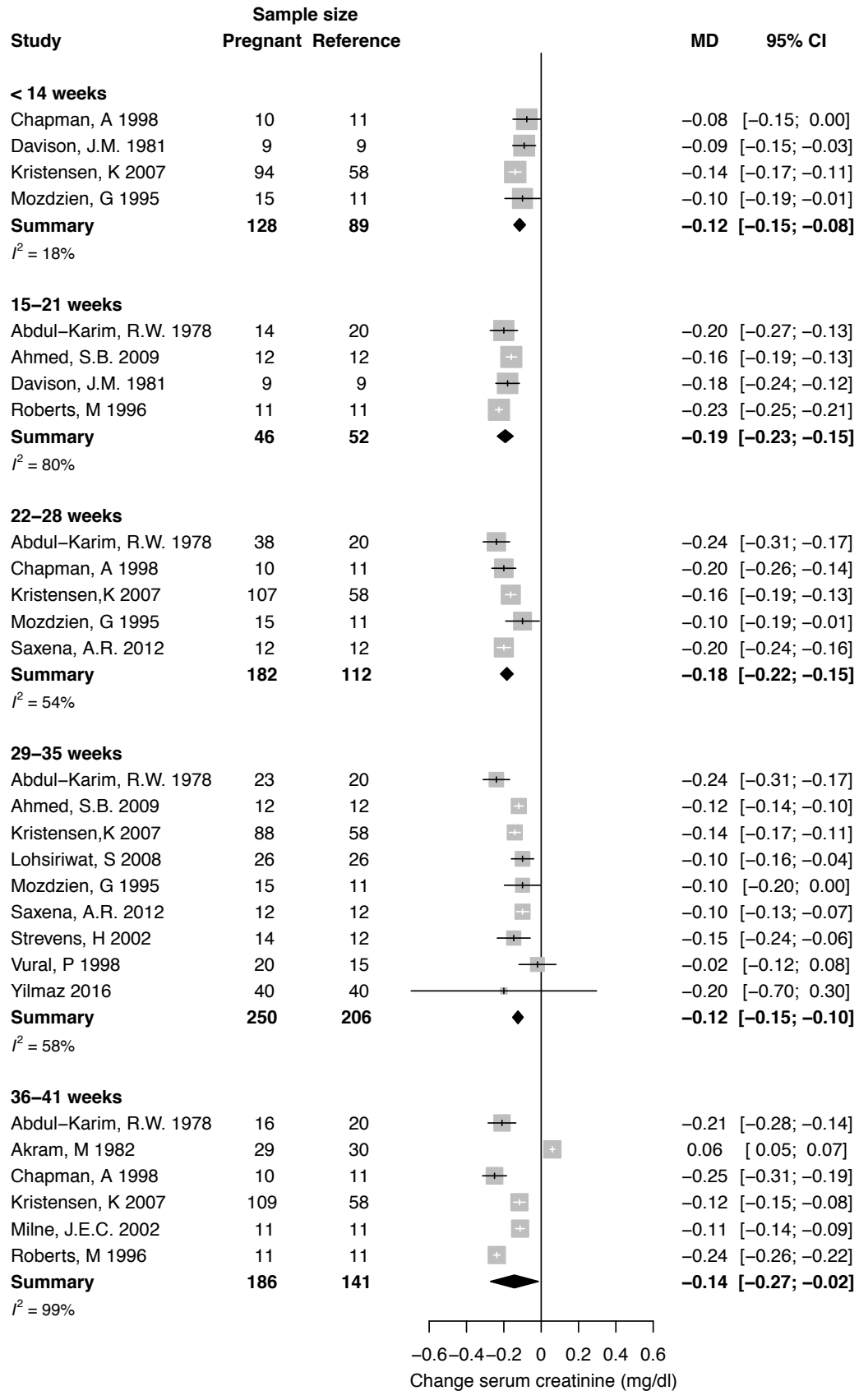

Figure 8 Forest plot of serum creatinine, in $\mathrm{mg} / \mathrm{dl}$, during physiologic pregnancies at $<14$ weeks, 15 - 21 weeks, 22 - 28 weeks, 29 - 35 weeks, and 36 - 41 weeks' gestation compared to reference values. Serum creatinine is reported as mean difference (MD) between pregnant and reference values, with $95 \% \mathrm{Cl}$. 


\section{Sample size}

Study

Pregnant Reference

29-35 weeks

Vural, P 1998

Yilmaz 2016

Summary

$I^{2}=13 \%$

36-41 weeks

Strevens, H 2002

Strevens, H 2002

Summary

$I^{2}=69 \%$

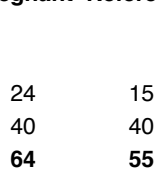

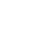

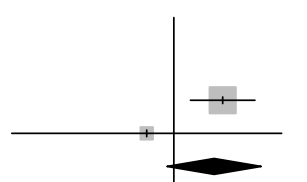

MD

$95 \% \mathrm{Cl}$

$0.18 \quad[0.06 ; 0.30]$

$-0.10[-0.60 ; 0.40]$

$0.15[-0.03 ; 0.32]$

$-0.08 \quad[-0.18 ; 0.03]$

$0.08 \quad[-0.06 ; 0.22]$

$-0.01[-0.16 ; 0.15]$

Figure 9 Forest plot of serum creatinine, in $\mathrm{mg} / \mathrm{dl}$, during hypertensive complicated pregnancies at $29-35$ and 36 - 41 weeks' gestation, compared to the reference values. Serum creatinine is reported as mean difference (MD) between pregnant and reference values, with 95\% Cl.

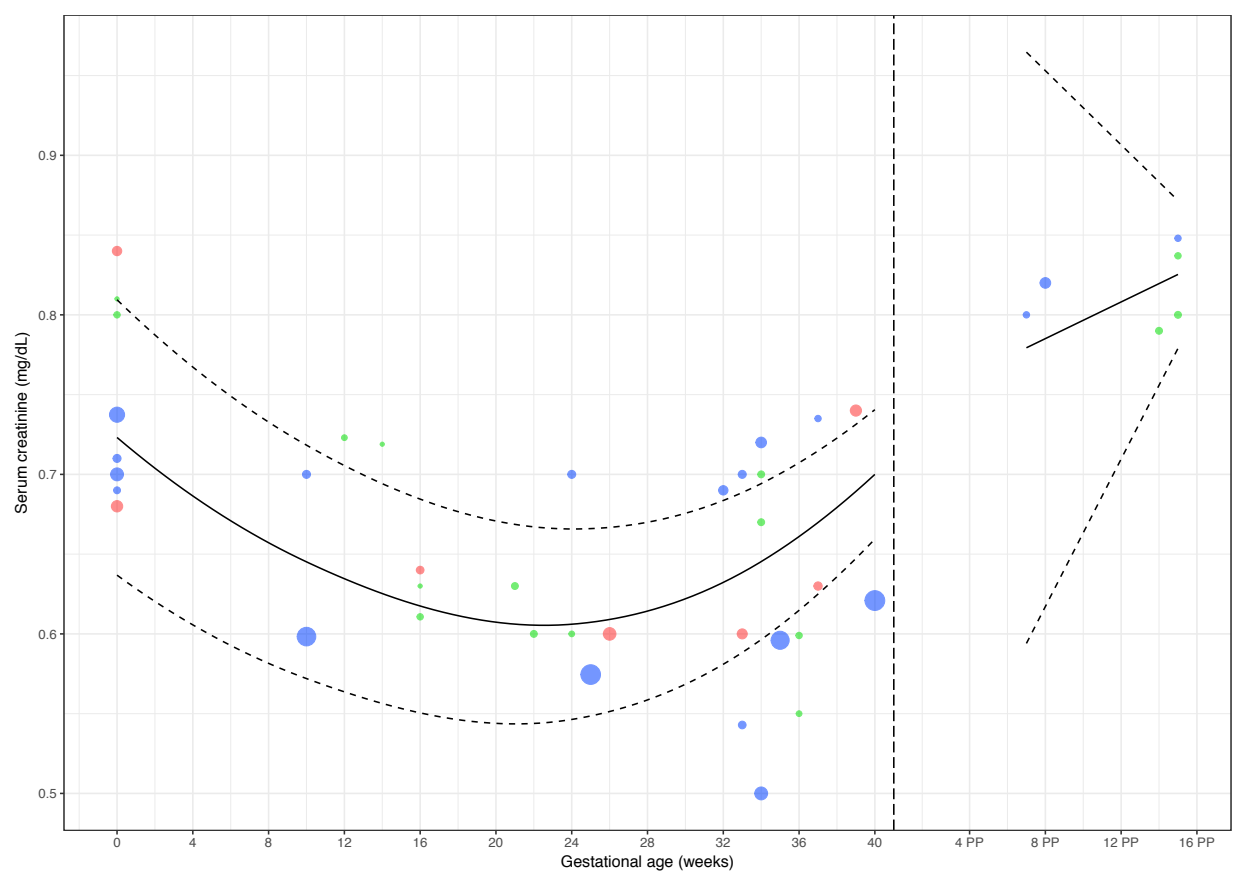

Figure 10 Decrease in serum creatinine, in mg/dl, during physiologic pregnancy. The circle size indicates the sample size of the point estimate. The colour of the circle refers to the quality assigned to the study: red $=\mathrm{LQ}$, green $=\mathrm{MQ}$, blue $=\mathrm{HQ}$. Curve fit is weighted by the inverse variance and plotted with $5^{\text {th }}$ and $95^{\text {th }}$ percentiles. The $50^{\text {th }}$ percentile is represented by a solid line. 


\section{DISCUSSION}

Kidney function, quantified by GFR, and either measured by inulin clearance or creatinine clearance, was increased during healthy uncomplicated pregnancy compared to nonpregnant conditions. The physiologic change in kidney function was not observed in women during a gestational hypertensive disease. Interestingly, GFR increase seems slightly less than previous literature details ${ }^{39}$. We found the largest increase during pregnancy to be 40 - 50\%, depending on the type of GFR measurement. Additionally, serum creatinine values were decreased during healthy uncomplicated pregnancies. Although the upper limit of the reference curve changed throughout gestation, from a clinical perspective, serum creatinine above $0.75 \mathrm{mg} / \mathrm{dl}$ ( $66 \mathrm{umol} / \mathrm{L}$ ) should be considered abnormal at all gestational ages.

GFR can be measured by the renal clearance of different substances. The ideal marker is a solute that is nontoxic, freely filtered at the glomerulus and neither secreted nor reabsorbed by the kidneys tubules. Despite the clinical importance of GFR, quantifying it is not without its difficulties. Over the years multiple endogenous and exogenous filtration markers have been used to evaluate kidney function ${ }^{40}$. Inulin is the most commonly used exogenous marker and considered the golden standard while creatinine is the most clinically used endogenous marker. Measuring GFR through inulin has the disadvantages of being a timeconsuming and complex procedure. Infusion protocols that use dextrose may be valid but usually overestimate GFR values due to hyperglycaemia. This may me particular relevant during pregnancy as maternal insulin resistance increases glucose availability ${ }^{41}$. The use of extra volume by infusion of saline, sometimes done when measuring GFR with inulin, can also positively affect GFR due to volume expansion. Because of the disadvantages of inulin, creatinine has become widely accepted to measure GFR even though it is less accurate. It is usually measured in a 24-hour urine collection in combination with serum creatinine to assess creatinine clearance. Inaccuracy of creatinine clearance is predominantly caused by the necessity to completely collect 24 hour urine and the bioavailability of creatinine quantities fluctuating as function of dietary intake and exercise induced muscle strain ${ }^{42,43}$.

The aggregated data used to build the GFR-CC curve show large heterogeneity between studies making it unfit to serve as a reference curve. Fitting curves using flexible regression techniques instead of a locally weighted curve, as we did to visualise reference ranges, could result in a better fit of all studies, it does however not result in a more reliable reference curve. 
Estimated GFR is calculated by serum creatinine and the use of different formulas that can take several factors into account, namely weight, age and ethnicity. The more commonly used formulas are Cockcroft-Gault (CG), Modification of Diet in Renal Disease (MDRD), and the more recent Chronic Kidney Disease Epidemiology Collaboration (CKD-EPI). During pregnancy (e)GFR can vary in accuracy and creatinine clearance, CG and MDRD are found to underestimate GFR ${ }^{23,25,44}$. It is also relevant to note that all of these formulas are based on large cohorts of non-pregnant patients with at least mild renal insufficiency ${ }^{45-47}$. eGFR is used frequently in daily practice but we did not find enough studies to include in our meta-analysis to provide an estimated difference during pregnancy. Measuring CKD-EPI, the more reliable estimation outside of pregnancy ${ }^{48}$, should be calculated before, during and after pregnancy in order to establish reference values relevant to clinicians in daily practice.

We found a significant difference in GFR both with inulin and creatinine clearance between women with uncomplicated pregnancies and women with a hypertensive complication in the second half of pregnancy; this is in agreement with known literature. The difference in changes of kidney function in pregnancies destined to be complicated by gestational hypertensive diseases could not be investigated because there were not enough studies in each interval. When assuming comparable body composition and glucose metabolism, a lower GFR and higher serum creatinine in gestational hypertensive pregnancies might originate from several mechanisms. Firstly, hypertension results in an increase in arterial tone as a result of an excess of vasoconstrictor over vasodilator influences in the systemic circulation ${ }^{49,50}$. The increased vascular tone causes in turn a higher renal vascular resistance that is responsible for a decrease in blood flow through the kidneys and therefore a decrease in GFR ${ }^{51}$.

Morphological changes in the kidney caused by preeclampsia, namely endotheliosis, can also result in a decrease in GFR. Secondly, it could be that some women, because of a pre-existing vulnerability, namely lesser nephrons, women with diabetes mellitus or immunologic disorders are more susceptible to damage to the kidneys and as a result a decrease in GFR. Since measuring GFR-IC during pregnancy is cumbersome and complex, GFR-CC is always considered to be the clinically more appropriate method to evaluate kidney function. However serum creatinine seems a reliable and stable measure with a low margin of error when compared to GFR-CC, which is also more discommodious for women. As serum creatinine is measured frequently during pregnancy it is an even more practical parameter. 
The following limitations of this study should be acknowledged. First, our meta-analysis was based on a relatively limited number of studies mostly due to the need of a non-pregnant reference within the studied population sample. This could have resulted in a lower statistical power, compared to the inclusion of all studies without reference samples, but it is thought to increase validity because of the use of identical measurement techniques. Second, authors reported participant's anthropometric data are often incomplete, making it impossible to analyse the contribution of these variables to the heterogeneity observed. Third, methodological shortcomings within and disparities amidst studies have resulted in limited data interpretation, which is reflected by our meta-regression analysis that exhibited a significant effect on the regression when study quality was taken into account. For MQ studies GFR-IC was on average $10 \mathrm{ml} / \mathrm{min}$ higher than HQ studies and for MQ GFR-CC studies was on averaged $22 \mathrm{ml} / \mathrm{min}$ lower when compared to HQ studies. A significant statistical association between GFR-IC course and reference measurement was also found. This difference was not found in GFR-CC and is possibly the effect of one single reference study that is relatively old and of a different methodological quality than other articles ${ }^{52}$. Also, according to Egger, the capacity to ascertain publication bias is restricted when meta-analysis consists solely of limited and/or small studies, and in this case both 22.

\section{CONCLUSION}

In healthy uncomplicated pregnancy kidney function is increased throughout, reflected by an increased GFR, measured by inulin or creatinine clearance, and a decrease in serum creatinine. In contrast, kidney function is decreased in hypertensive gestation. Although the upper limit of the reference curve changes throughout gestation, from a clinical perspective, serum creatinine above $0.75 \mathrm{mg} / \mathrm{dl}$ ( $66 \mathrm{umol} / \mathrm{L}$ ) should be considered abnormal at all gestational ages. 


\section{REFERENCES}

1. Bosio PM, McKenna PJ, Conroy R, O'Herlihy C. Maternal Central Hemodynamics in Hypertensive Disorders of Pregnancy. Obstet. Gynecol. 1999;94:978-984.

2. Morris NH, Eaton BM, Dekker G. Nitric oxide, the endothelium, pregnancy and pre-eclampsia. Br. J. Obstet. Gynaecol. Jan 1996;103(1):4-15.

3. Conrad KP. Mechanisms of Renal Vasodilatation and Hyperfiltration During Pregnancy: Current Perspectives and Potentia; Implications for Preeclampsia. Endothelium. 2005 2005;12:57-62.

4. Odutayo A, Hladunewich M. Obstetric nephrology: renal hemodynamic and metabolic physiology in normal pregnancy. Clin. J. Am. Soc. Nephrol. Dec 2012;7(12):2073-2080.

5. Cheung KL, Lafayette RA. Renal physiology of pregnancy. Adv. Chronic Kidney Dis. May 2013;20(3):209-214.

6. Davison JM, Dunlop W. Renal hemodynamics and tubular function in normal human pregnancy. Kidney Int. 1980;18(2):152-161.

7. Cornelis T, Odutayo A, Keunen J, Hladunewich M. The kidney in normal pregnancy and preeclampsia. Semin. Nephrol. Jan 2011;31(1):4-14.

8. Lafayette RA, Druzin M, Dibley R, et al. Nature of glomerular dysfunction in pre-eclampsia. Kidney Int. 1998;54:1240-1249.

9. Irons DW, Baylis PH, Davison JM. Effect of atrial natriuretic peptide on renal hemodynamics and sodium excretion during human pregnancy. Am. J. Physiol. Jul 1996;271(1 Pt 2):F239-242.

10. Milne JE, Lindheimer MD, Davison JM. Glomerular heteroporous membrane modeling in third trimester and postpartum before and during amino acid infusion. Am. J. Physiol. Renal Physiol. Jan 2002;282(1):F170-175.

11. Sturgiss SN, Wilkinson R, Davison JM. Renal reserve during human pregnancy. Am. J. Physiol. 1996;271:16-20.

12. Davison JM, Hytten FE. Glomerular filtration during and after pregnancy. Br. J. Obstet. Gynaecol. 1974;81:588595.

13. Chapman AB, Abraham WT, Zamudio S, et al. Temporal relationships between hormonal and hemodynamic changes in early human pregnancy. Kidney Int. Dec 1998;54(6):2056-2063.

14. Davison JM, MC. N. Serial changes in 24 hour creatinine clearance during normal menstrual cycles and the first trimester of pregnancy. Br. J. Obstet. Gynaecol. 1981;88(1):10-17.

15. Hayden JA, L.Cartwright DAvdWJ, Cote P, Bombardier C. Assessing Bias in Studies of Prognostic Factors. Ann. Intern. Med. 2013;158:280-286.

16. Abudu OO, Sofola OA. Plasma volume in normal pregnant Nigerian primigravidae. Int. J. Gynaecol. Obstet. Apr 1985;23(2):137-142.

17. Higgins J, Green S. Cochrane Handbook for Systematic Reviews of Interventions Version 5.1.0 [updated March 2011]. Cochrane Collab 2011, :Available from http//handbook.cochrane.org.

18. DerSimonian R, Laird N. Meta-Analysis in Clinical Trials. Control. Clin. Trials. 1986;7:177-188.

19. Team TRC. R: A Language and Environment for Statistical Computing. 2015.

20. Schwarzer G. Meta: General package for meta-analysis. R package version 4.3-2 2015.

21. Higgins J, Thompson SG, Deeks JJ, Altman DG. Measuring inconsistency in meta-analyses. Br. Med. J. 2003;327:557-560.

22. Egger M, Smith GD, Schneider M, Minder C. Bias in meta-analysis detected by a simple, graphical test. Br. Med. J. 1997;316:629-634.

23. Ahmed SB, Bentley-Lewis R, Hollenberg NK, Graves SW, Seely EW. A comparison of prediction equations for estimating glomerular filtration rate in pregnancy. Hypertens. Pregnancy. 2009;28(3):243-255.

24. Platts JK, Meadows $P$, Jones R, Harvey JN. The relation between tissue kallikrein excretion rate, aldosterone and glomerular filtration rate in human pregnancy. Br. J. Obstet. Gynaecol. Feb 2000;107(2):278-281.

25. Smith MC, Moran P, Ward MK, Davison JM. Assessment of glomerular filtration rate during pregnancy using the MDRD formula. Br. J. Obstet. Gynaecol. Jan 2008;115(1):109-112.

26. Brandstetter F, Schuller E. Studies on liver and kidney function in late toxemia. Arch Gynakol. 1955;186:189192.

27. deAlvarez RR, Wash S. Glomerular filtration rates, renal plasma flow, and sodium and water retention in pregnancy toxemia. Am. J. Obstetrics and Gynaecology. Dec 1950;60(5):1051-1067.

28. Varga I, Rigó J, Somos P, Joó JG, B N. Analysis of maternal circulation and renal function in physiologic pregnancies; parallel examinations of the changes in the cardiac output and the glomerular filtration rate. J. Matern. Fetal Med. 2000;9(2):97-104. 
29. Davison JM, Noble MCB. Serial changes in 24 hour creatinine clearance during normal menstrual cycles and the first trimester of pregnancy. Br. J. Obstet. Gynaecol. 1981;88:10-17.

30. Kristensen K, Lindstrom V, Schmidt C, et al. Temporal changes of the plasma levels of cystatin C, beta-trace protein, beta2-microglobulin, urate and creatinine during pregnancy indicate continuous alterations in the renal filtration process. Scand. J. Clin. Lab. Invest. 2007;67(6):612-618.

31. Strevens H, Wide-Swensson D, Torffvit O, Grubb A. Serum cystatin C for assessment of glomerular filtration rate in pregnant and non-pregnant women. Indications of altered filtration process in pregnancy. Scand. J. Clin. Lab. Invest. 2009;62(2):141-148.

32. Barden A, Beilin LJ, Burke V, Ritchie J, Michael CA, Walters BN. Relationships between plasma endothelin 1 and prostacyclin in normal and preeclamptic pregnancy. Hypertens. Pregnancy. 1996;15(1):25-38.

33. Buttermann K. Clearance-Untersuchungen in der normalen und pathologischen Schwangerschaft Zugleich eine kritische Beurteilung des Verfahrens. Archly fiir Gynakologie. 1958;190:448-492.

34. Moran P, Baylis PH, Lindheimer MD, Davison JM. Glomerular ultrafiltration in normal and preeclamptic pregnancy. J. Am. Soc. Nephrol. Mar 2003;14(3):648-652.

35. Schneider F, Lutun P, Baldauf J-J, et al. Plasma cyclic GMP concentrations and their relationship with changes of blood pressure levels in pre-eclampsia. Acta Obstet. Gynecol. Scand. 1996;75:40-44.

36. Vural P, Akgül C, Canbaz M. Urinary PGE2 and PGF2 Levels and Renal Functions in Preeclampsia. Gynecol. Obstet. Invest. 1998;45:237-241.

37. Wang MX, Brown MA, Buddle ML, Carlton MA, Cario GM, Whitworth JA. Endothelin excretion in hypertensive pregnancy. Relationship to glomerular filtration rate, blood pressure, and sodium excretion. Am. J. Hypertens. Apr 1994;7(4 Pt 1):308-313.

38. Yilmaz ZV, Akkas E, Yildirim T, Yilmaz R, Erdem Y. A novel marker in pregnant with preeclampsia: renalase. J. Matern. Fetal Neonatal Med. Apr 2017;30(7):808-813.

39. Lindheimer MD, Davison JM, Katz Al. The kidney and hypertension in pregnancy: twenty exciting years. Semin. Nephrol. Mar 2001;21(2):173-189.

40. Lamb EJ, Stevens PE. Estimating and measuring glomerular filtration rate: methods of measurement and markers for estimation. Curr. Opin. Nephrol. Hypertens. May 2014;23(3):258-266.

41. Sonagra AD, Biradar SM, K D, Murthy DSJ. Normal pregnancy- a state of insulin resistance. J Clin Diagn Res. Nov 2014;8(11):CC01-03.

42. Stevens LA, Levey AS. Measurement of kidney function. Med. Clin. North Am. May 2005;89(3):457-473.

43. Stevens LA, Coresh J, Greene T, Levey AS. Assessing kidney function--measured and estimated glomerular filtration rate. N. Engl. J. Med. Jun 8 2006;354(23):2473-2483.

44. Koetje PM, Spaan J, Kooman JP, Spaanderman MEA, Peeters LLH. Pregnancy reduces the accuracy of the estimated glomerular filtration rate based on Cockroft-Gault and MDRD formulas. Reprod. Sci. May 2011;18(5):456-462.

45. Levey AS, Stevens LA, Schmid CH, et al. A new equation to estimate glomerular filtration rate. Ann. Intern. Med. May 05 2009;150(9):604-612.

46. Levey AS, Bosch JP, Lewis JB, Greene T, Rogers N, Roth D. A more accurate method to estimate glomerular filtration rate from serum creatinine: a new prediction equation. Modification of Diet in Renal Disease Study Group. Ann. Intern. Med. Mar 16 1999;130(6):461-470.

47. Cockcroft DW, Gault MH. Prediction of creatinine clearance from serum creatinine. Nephron. 1976;16(1):31 41.

48. Inker LA, Shaffi K, Levey AS. Estimating glomerular filtration rate using the chronic kidney disease-epidemiology collaboration creatinine equation: better risk predictions. Circ. Heart Fail. May 01 2012;5(3):303-306.

49. Thadhani RI, Maynard SE, Glassock RJ, Sterns RH, Eckler K. Renal and urinary tract physiology in normal pregnancy. Www.uptodate.com. 2016

50. Lafayette R. The kidney in preeclampsia. Kidney Int. Mar 2005;67(3):1194-1203.

51. Karumanchi SA, Maynard SE, Stillman IE, Epstein FH, Sukhatme VP. Preeclampsia: a renal perspective. Kidney Int. Jun 2005;67(6):2101-2113.

52. Buttermann K. Clearance studies in normal and pathological pregnancy, with a critical evaluation of the method. Arch Gynakol. 1958;190(5):448-492.

53. Abdul-Karim RW, Harris JE, Beydoun SN, Cuenca VG. Endogenous creatinine clearance during pregnancy. II. Variations in normal standards based on methodology. Obstet. Gynecol. Apr 1978;51(4):431-432.

54. Akram M, Akhtar MS, Waqar N. Glomerular filtration rate and levels of serum and urinary proteins in nonpregnant, pregnant and post puerperal Pakistani women. J. Pak. Med. Assoc. Nov 1982;32(11):257-262. 
55. Barron WM, Lindheimer MD. Effect of oral protein loading on renal hemodynamics in human pregnancy. Am. J. Physiol. 1995;369:888-895.

56. Davison JM, Dunlop W, M. E. 24-Hour creatinine clearance during the third trimester of normal pregnancy. Br. J. Obstet. Gynaecol. 1980;97(2):106-109.

57. Dunlop W. Investigations into the influence of posture on renal plasma flow during late pregnancy. Br. J. Obstet. Gynaecol. 1975;82:588-590.

58. Dunlop W. Serial changes in renal haemodynamics during normal human pregnancy. Br. J. Obstet. Gynaecol. 1981;88(1):1-9.

59. Gibson HM. Plasma volume and glomerular filtration rate in pregnancy and their relation to differences in fetal growth. Br J Obstet Gynaecol. 1973;80:1067-1074.

60. Lohsiriwat S, Imrittha N. Effect of posture on creatinine clearance in late pregnancy and after pregnancy. J. Obstet. Gynaecol. Res. 2008;34(3):337-342.

61. Mozdzien G, Schinninger M, Zazgornik J. Kidney function and electrolyte metabolism in healthy pregnant women. Wien. Med. Wochenschr. 1995;145(1):12-17.

62. Roberts M, Lindheimer MD, Davison JM. Altered glomerular permselectivity to neutral dextrans and heteroporous membrane modeling in human pregnancy. Am. J. Physiol. Feb 1996;270(2 Pt 2):F338-343.

63. Saxena AR, Ananth Karumanchi S, Fan SL, et al. Correlation of cystatin-C with glomerular filtration rate by inulin clearance in pregnancy. Hypertens. Pregnancy. 2012;31(1):22-30.

64. Wright A, Steele P, Bennett JR, Watts G, Polak A. The urinary excretion of albumin in normal pregnancy. Br J Obstet Gynaecol. May 1987;94(5):408-412. 


\begin{tabular}{|c|c|}
\hline Component & PubMed \\
\hline Pregnancy & $\begin{array}{l}\text { "Pregnancy" [Mesh] OR "pregnancy" [Title/Abstract] OR "pregnancies" } \\
\text { [Title/Abstract] OR "pregnant" [Title/Abstract] OR "gestation" [Title/ } \\
\text { Abstract] OR "gestations" [Title/Abstract] OR "gestational" [Title/Abstract] } \\
\text { OR "gravidity" [Mesh] OR "gravidity" [Title/Abstract] OR "gravidities" [Title/ } \\
\text { Abstract] OR "gravid" [Title/Abstract] }\end{array}$ \\
\hline $\begin{array}{l}\text { Pregnancy-induced } \\
\text { hypertension }\end{array}$ & $\begin{array}{l}\text { "Hypertension, pregnancy-induced" [Mesh] OR "pregnancy induced } \\
\text { hypertension" [Title/Abstract] OR "pregnancy associated hypertension" } \\
\text { [Title/Abstract] OR "PIH" [Title/Abstract] OR "hypertensive pregnancy" } \\
\text { [Title/Abstract] OR "pregnancy hypertension" [Title/Abstract] OR } \\
\text { "gestational hypertension" [Title/Abstract] OR "HELLP syndrome" [Mesh] } \\
\text { OR "HELLP" [Title/Abstract] OR "Hemolysis, Elevated Liver Enzymes, } \\
\text { Lowered Platelets" [Title/Abstract] }\end{array}$ \\
\hline $\begin{array}{l}\text { Fetal growth } \\
\text { retardation }\end{array}$ & $\begin{array}{l}\text { "Fetal Growth Retardation" [Mesh] OR "foetal growth retardation" [Title/ } \\
\text { Abstract] OR "fetal growth restriction" [Title/Abstract] OR "FGR" [Title/ } \\
\text { Abstract] OR "intrauterine growth retardation" [Title/Abstract] OR } \\
\text { "intrauterine growth restriction" [Title/Abstract] OR "IUGR" [Title/Abstract] } \\
\text { OR "Infant, Small for Gestational Age" [Mesh] OR "small for gestational age" } \\
\text { [Title/Abstract] OR "SGA" [Title/Abstract] }\end{array}$ \\
\hline Preeclampsia & $\begin{array}{l}\text { "Pre-eclampsia" [Mesh] OR "pre-eclampsia" [Title/Abstract] OR } \\
\text { "preeclampsia" [Title/Abstract] OR "preeclamptic" [Title/Abstract] OR } \\
\text { "pre-eclamptic" [Title/Abstract] or "PE" [Title/Abstract] OR "Eclampsia" } \\
\text { [Mesh] OR "eclampsia" [Title/Abstract] OR "eclampsias" [Title/Abstract] OR } \\
\text { "eclamptic" [Title/Abstract] OR "toxemia" [Title/Abstract] OR "toxemias" } \\
\text { [Title/Abstract] }\end{array}$ \\
\hline $\begin{array}{l}\text { Gestational } \\
\text { diabetes }\end{array}$ & $\begin{array}{l}\text { "Diabetes, Gestational" [Mesh] OR "pregnancy induced diabetes" } \\
\text { [Title/Abstract] OR "gestational diabetes" [Title/Abstract] OR "diabetes } \\
\text { gravidarum" [Title/abstract] }\end{array}$ \\
\hline Kidney function & $\begin{array}{l}\text { "Glomerular Filtration Rate"[Mesh] OR "Kidney Function Tests"[Mesh] } \\
\text { OR "Renal Elimination"[Mesh] OR "glomerular filtration"[title/abstract] OR } \\
\text { "GFR"[title/abstract] OR "eGFR"[title/abstract] OR "kidney function"[title/ } \\
\text { abstract] OR "renal function"[title/abstract] OR "renal elimination"[title/ } \\
\text { abstract] OR "kidney elimination"[title/abstract] OR "creatinine } \\
\text { clearance"[title/abstract] OR "CCR"[title/abstract] OR "CrCl"[title/abstract] } \\
\text { OR "eCCR"[title/abstract] OR "inulin clearance"[title/abstract] OR "cystatin C } \\
\text { clearance"[title/abstract] OR "urea clearance"[title/abstract])) }\end{array}$ \\
\hline
\end{tabular}

Table 1 Literature search strategy for PubMed (NCBI) and Embase (Ovid) databases. 


\section{Embase}

exp pregnancy/ or exp gravidity/ or exp gestation/ or (pregnancy or pregnancies or pregnant or gestation or gestations or gestational or gravidity or gravidities or gravid).ti,ab.

exp maternal hypertension/ or exp HELLP syndrome/ or (pregnancy induced hypertension or pregnancy associated hypertension or PIH or hypertensive pregnancy or pregnancy hypertension or gestational hypertension or HELLP or Hemolysis, Elevated Liver Enzymes, Lowered Platelets).ti,ab.

exp intrauterine growth retardation/ or exp small for date infant/ or (fetal growth retardation or fetal growth restriction or FGR or intrauterine growth retardation or intrauterine growth restriction or IUGR or small for gestational age or SGA ).ti,ab.

exp preeclampsia/ or exp eclampsia/ or (pre-eclampsia or preeclampsia or pre-eclamptic or preeclamptic or PE or eclampsia or eclampsias or eclamptic or toxemia or toxemias).ti,ab.

exp pregnancy diabetes mellitus/ or (pregnancy induced diabetes or gestational diabetes or diabetes gravidarum).ti,ab.

Exp glomerular filtration/ or exp glomerular filtration rate/ or exp kidney function test/ or exp kidney function/ or exp creatinine clearance/ or exp inulin clearance/ or (glomerular filtration or GFR or eGFR or kidney function or renal function or renal elimination or kidney elimination or creatinine clearance or CCR or CrCl or eCCR or inulin clearance or cystatin c clearance or urea clearance).ti,ab. 


\begin{tabular}{|c|c|c|c|c|c|c|c|c|c|c|}
\hline \multirow[b]{3}{*}{ Study } & & & & & \multicolumn{4}{|c|}{ Non-pregnant } & \multicolumn{2}{|c|}{ Parity / gravidity (n) } \\
\hline & \multicolumn{2}{|c|}{$\begin{array}{l}\text { Subjects } \\
\text { (n) }\end{array}$} & \multicolumn{2}{|c|}{$\begin{array}{l}\text { Age } \\
\text { (years) }\end{array}$} & \multicolumn{2}{|c|}{$\begin{array}{l}\text { Weight } \\
(\mathrm{kg})\end{array}$} & \multicolumn{2}{|c|}{$\begin{array}{l}\text { Height } \\
(\mathrm{cm})\end{array}$} & \multicolumn{2}{|c|}{ Nulli } \\
\hline & $\mathrm{R}$ & $P$ & $\mathrm{R}$ & $\mathrm{P}$ & $\mathrm{R}$ & $P$ & $\mathrm{R}$ & $P$ & $\mathrm{R}$ & $\mathrm{P}$ \\
\hline Abdul-Karim $1978{ }^{53}$ & 20 & 91 & - & - & - & - & - & - & - & - \\
\hline Ahmed 2009 & 12 & 12 & - & - & 65 & 65 & 164 & 164 & - & - \\
\hline Akram 198254 & 30 & 29 & 28.5 & 29.7 & - & - & - & - & - & - \\
\hline Barden 1996 & 24 & 28 & 27.3 & 26.8 & - & - & - & - & - & - \\
\hline Barron 199555 & 22 & 22 & 28.7 & 28.7 & - & - & - & - & - & - \\
\hline Buttermann 1958 & 11 & 72 & - & - & - & - & - & - & - & - \\
\hline Chapman 1998 & 11 & 10 & 30.9 & 30.9 & 61.5 & - & - & - & 9 & - \\
\hline Davison 1974 & 10 & 10 & 28.7 & 28.7 & 56.1 & 56.1 & 160 & 160 & - & - \\
\hline Davison 1981 & 9 & 9 & 26.4 & 26.4 & 58.6 & 58.6 & 162 & 162 & 2 & 2 \\
\hline Davison 198056 & 10 & 10 & & & 61.8 & 61.8 & 164 & 164 & - & - \\
\hline Dunlop $1975^{57}$ & 18 & 18 & - & - & - & - & - & - & - & - \\
\hline Dunlop 198158 & 25 & 25 & 28.6 & 28.6 & 62.8 & 62.8 & 163 & 163 & - & - \\
\hline Gibson 197359 & 9 & 9 & 28.1 & 28.1 & 56.4 & 56.4 & 157 & 157 & - & - \\
\hline Irons 1996 & 12 & 12 & - & - & - & - & - & - & - & - \\
\hline Kristensen 2007 & 58 & 398 & 25 & 32 & - & - & - & - & 49 & 177 \\
\hline Lohsiriwat 200860 & 26 & 26 & 25.1 & 25.1 & 63.15 & 63.15 & - & - & - & - \\
\hline Milne 2002 & 11 & 11 & 27.1 & 27.1 & - & - & - & - & - & - \\
\hline Moran 2003 & 13 & 13 & - & - & - & - & - & - & - & - \\
\hline Mozdzien 199561 & 11 & 15 & 23 & 23 & - & - & 164 & 164 & - & - \\
\hline Roberts $1996{ }^{62}$ & 11 & 11 & 31 & 31 & - & - & 164 & 164 & 6 & 6 \\
\hline Saxena $2012^{63}$ & 12 & 12 & 28.7 & 28.7 & - & - & 163 & 164 & - & - \\
\hline Schneider 1996 & 6 & 16 & 23.5 & 28.5 & - & - & - & - & - & - \\
\hline Smith 2008 & 23 & 24 & - & - & - & - & - & - & - & - \\
\hline Strevens 2002 & 12 & 14 & 27 & 30 & 58.8 & - & 168 & 163 & - & - \\
\hline Sturgiss 1996 & 7 & 7 & 31.0 & 31.0 & 63.3 & 63.3 & 159 & 159 & - & - \\
\hline Vural 1998 & 15 & 20 & - & - & - & - & - & - & - & 20 \\
\hline Wang 1994 & 26 & 42 & 27 & 29 & - & - & - & - & - & - \\
\hline Wright 198764 & 9 & 31 & - & - & - & - & & - & - & - \\
\hline Yilmaz 2016 & 40 & 40 & 27.6 & 26.0 & - & - & - & - & - & - \\
\hline
\end{tabular}

Table 2 Characteristics of included studies, physiological pregnancies, arranged by first author and year of publication. Characteristics are presented by means reported by the study. Gravidity is underlined and parity is in Italic. Reference (R) of kidney function was measured in pre-pregnancy $(\mathrm{PC})$, non-pregnant (NP) or postpartum (PP) controls. During physiologic pregnancy (P) kidney function was measured at different durations of amenorrhea. $C C=$ creatinine clearance. 


\begin{tabular}{|c|c|c|c|c|c|c|}
\hline \multicolumn{7}{|c|}{ Parity / gravidity (n) } \\
\hline \multicolumn{2}{|c|}{ Primi } & \multicolumn{3}{|c|}{ Multi } & \multirow{2}{*}{$\begin{array}{l}\text { Duration of } \\
\text { amenorrhea at } \\
\text { measurement } \\
\text { (weeks) }\end{array}$} & \multirow{2}{*}{$\begin{array}{l}\text { Methods GFR (inulin and/or } \\
\text { duration of collection for } \mathrm{CC} \text { ), serum } \\
\text { creatinine }\end{array}$} \\
\hline $\mathrm{R}$ & P & $\mathrm{R}$ & $\mathrm{P}$ & Baseline & & \\
\hline- & - & - & - & NP & $16,26,33,37$ & Serum creatinine \\
\hline- & - & - & - & PP (15 weeks) & 21,34 & GFR (inulin, 24h CC), serum creatinine \\
\hline- & - & - & - & NP & $39,10 \mathrm{PP}$ & GFR (24h CC), serum creatinine \\
\hline- & 13 & - & 15 & NP & 30 & GFR (24h CC) \\
\hline- & - & - & - & PP (44 weeks) & 30 & GFR (inulin and 24h CC) \\
\hline- & - & - & - & NP & $11,23,35$ & GFR (inulin and 24h CC) \\
\hline- & - & - & - & $P C$ & $12,24,36$ & Serum creatinine \\
\hline 3 & 3 & 7 & 7 & PP (10 weeks) & $17,27,37$ & GFR (inulin and 24h CC), \\
\hline 7 & 7 & - & - & $P C$ & 14,16 & GFR (24h CC), serum creatinine \\
\hline 3 & 3 & 7 & 7 & PP (10 weeks) & 26 & GFR (24h CC) \\
\hline- & - & - & - & PP (8 weeks) & 37 & GFR (inulin) \\
\hline 10 & 10 & 15 & 15 & PP (8 weeks) & $16,26,36$ & GFR (inulin) \\
\hline$\underline{7}$ & $\underline{7}$ & $\underline{2}$ & $\underline{2}$ & PP (12 weeks) & 20,28 & GFR (inulin) \\
\hline 12 & 12 & - & - & PP (16 weeks) & 32 & GFR (inulin) \\
\hline- & - & - & - & NP & $10,25,35,40$ & Serum creatinine \\
\hline- & - & - & - & PP (9 weeks) & 34 & GFR (24h CC), serum creatinine \\
\hline- & - & - & - & PP (16 weeks) & 37 & GFR (inulin), serum creatinine \\
\hline- & - & - & - & PP (20 weeks) & 37 & GFR (inulin) \\
\hline 6 & 6 & 9 & 9 & PP (8 weeks) & $10,24,33$ & GFR (24h CC), serum creatinine \\
\hline- & - & - & - & PP (16 weeks) & 16,36 & GFR (inulin), serum creatinine \\
\hline- & - & - & - & PP (16 weeks) & 22,34 & GFR (inulin, 24h CC), serum creatinine \\
\hline$\underline{0}$ & 11 & - & - & NP & 33 & GFR (24h CC) \\
\hline- & - & - & - & PP (8 weeks) & 13,36 & GFR (inulin) \\
\hline- & - & - & - & NP & 33 & Serum creatinine \\
\hline- & - & - & - & PP (22 weeks) & 18,35 & GFR (inulin) \\
\hline- & - & - & - & NP & 32 & GFR (24h CC), serum creatinine \\
\hline- & - & - & - & NP & 34 & GFR (24h CC) \\
\hline 17 & 17 & - & - & PP (6 weeks) & $14,28,36$ & GFR (2h CC) \\
\hline- & - & - & - & NP & 34 & GFR (24h CC),serum creatinine \\
\hline
\end{tabular}




\begin{tabular}{|c|c|c|c|c|c|c|c|c|c|c|}
\hline \multirow[b]{3}{*}{ Study } & & & & & \multicolumn{4}{|c|}{ Non-pregnant } & \multicolumn{2}{|c|}{ Parity/gravidity (n) } \\
\hline & \multicolumn{2}{|c|}{ Subjects (n) } & \multicolumn{2}{|c|}{ Age (years) } & \multicolumn{2}{|c|}{ Weight (kg) } & \multicolumn{2}{|c|}{ Height (cm) } & \multicolumn{2}{|l|}{ Nulli } \\
\hline & $\mathrm{R}$ & $\mathrm{CP}$ & $\mathrm{R}$ & $\mathrm{CP}$ & $\mathrm{R}$ & $\mathrm{CP}$ & $\mathrm{R}$ & $\mathrm{CP}$ & $\mathrm{R}$ & $\mathrm{CP}$ \\
\hline Barden 1996 & 24 & 20 & 27.3 & 27.4 & - & - & - & - & - & - \\
\hline Buttermann 1958 & 11 & 33 & - & - & - & - & - & - & - & - \\
\hline Moran 2003 & 5 & 10 & - & - & - & - & - & - & - & - \\
\hline Schneider 1996 & 6 & 16 & 23.5 & 28.6 & - & - & - & - & - & - \\
\hline Strevens 2002 & 12 & 24 & 27 & 31 & 58.8 & - & 168 & 164 & - & - \\
\hline Vural 1998 & 15 & 24 & - & - & - & - & - & - & - & 24 \\
\hline Wang 1994 & 26 & 37 & 27 & 27 & - & - & - & - & - & - \\
\hline Yilmaz 2016 & 40 & 40 & 27.6 & 27.9 & - & - & - & - & - & - \\
\hline
\end{tabular}

Table 3 Characteristics of included studies, hypertensive complicated pregnancies, arranged by first author and year of publication. Characteristics are presented by means reported by the study. Gravidity is underlined and parity is in Italic. Parity/gravity: Nulli, Primi, Multi.Reference (R) kidney function was measured in non-pregnant (NP) or postpartum (PP) controls. During hypertensive complicated pregnancy gestational (CP) kidney function was measured between 30 and 37 weeks. $\mathrm{CC}=$ creatinine clearance. 


\begin{tabular}{|c|c|c|c|c|c|c|}
\hline \multicolumn{7}{|c|}{ Parity/gravidity (n) } \\
\hline \multicolumn{2}{|c|}{ Primi } & \multicolumn{3}{|l|}{ Multi } & \multirow{2}{*}{$\begin{array}{l}\text { Duration } \\
\text { amenorrhea } \\
\text { at measurement } \\
\text { (weeks) }\end{array}$} & \multirow{2}{*}{$\begin{array}{l}\text { Methods } \\
\text { GFR (inulin and/or duration } \\
\text { of collection for CC), serum } \\
\text { creatinine }\end{array}$} \\
\hline $\mathrm{R}$ & $\mathrm{CP}$ & $\mathrm{R}$ & $\mathrm{CP}$ & Baseline & & \\
\hline- & 9 & - & 11 & NP & 30 & GFR (24h CC) \\
\hline- & & & & NP & 35 & GFR (inulin and 24h CC) \\
\hline- & - & - & - & PP (20 weeks) & 37 & GFR (inulin) \\
\hline 0 & 11 & - & - & NP & 33 & GFR (not stated h CC) \\
\hline- & - & - & - & NP & 36 & Serum creatinine \\
\hline- & - & - & - & NP & 33 & GFR (24h CC), serum creatinine \\
\hline- & - & - & - & NP & 36 & GFR (24h CC) \\
\hline- & - & - & - & NP & 35 & GFR (24h CC), serum creatinine \\
\hline
\end{tabular}




\begin{tabular}{|c|c|}
\hline Study & Loadingdose \\
\hline Ahmed 2009 & Inulin $50 \mathrm{mg} / \mathrm{kg}$ and PAH $8 \mathrm{mg} / \mathrm{kg}$ \\
\hline Barron 1995 & Inulin 50mg/kg and PAH 8 mg/kg \\
\hline Buttermann 1958 & Inulin $10 \mathrm{ml} 10 \%$ and PAH $4 \mathrm{ml} 20 \%$ in 5 minutes \\
\hline Davison 1974 & $\begin{array}{l}\text { Dextrose } 10 \% \text { and } 1 \mathrm{~g} \text { of inulin } \\
\text { at a rate of } 16 \mathrm{ml} / \mathrm{min} \text { for } 10 \text { minutes } \\
\text { (to reach plasma levels between } 150-300 \mathrm{mg} / 100 \mathrm{ml} \text { ) }\end{array}$ \\
\hline Dunlop 1975 & not stated \\
\hline Dunlop 1981 & $\begin{array}{l}\text { Inulin } 400 \mathrm{mg} \text { and } \mathrm{PAH} 160 \mathrm{mg} \\
\text { at a rate of } 16 \mathrm{ml} / \mathrm{min} \text { for } 10 \text { minutes }\end{array}$ \\
\hline Gibson 1973 & not stated \\
\hline Irons 1996 & Inulin 25\% $10 \mathrm{ml}$ and PAH 20\% $2 \mathrm{ml}$ \\
\hline Milne 2002 & $\begin{array}{l}\text { Inulin 25\% } 10 \mathrm{ml} \text {, Dextran 10\% } 48 \mathrm{ml} \text { and PAH 20\% 2dl administered over } \\
10 \text { minutes }\end{array}$ \\
\hline Moran 2003 & $\begin{array}{l}\text { Inulin unknown \% } 10 \mathrm{ml} \text {, Dextran 10\% } 48 \mathrm{ml} \text { and PAH 20\% } 2 \mathrm{ml} \\
\text { administered over } 10 \text { minutes }\end{array}$ \\
\hline Roberts 1996 & Inulin 25\% $10 \mathrm{ml}$,Dextran 10\% $48 \mathrm{ml}$ and PAH 20\% $2 \mathrm{ml}$ administered \\
\hline Saxena 2012 & Inulin 50 mg/kg \\
\hline Smith 2008 & Inulin 25\% $10 \mathrm{ml}$ and $48 \mathrm{ml} 0.9 \% \mathrm{NaCl}$ over 5 minutes \\
\hline Sturgiss 1996 & Inulin $2.5 \mathrm{~g}$, PAH $400 \mathrm{mg}$ and $50 \mathrm{ml}$ of $0.9 \% \mathrm{NaCl}$ over $5 \mathrm{~min}$ \\
\hline
\end{tabular}

Table 4 Description of measurement methods used in studies with glomerular filtration rate measured by inulin.

$\mathrm{PAH}=\mathrm{p}$-aminohippurate, $\mathrm{NaCl}=0.9 \%$ saline . 


\section{Infusion rate}

Inulin at $30 \mathrm{mg} / \mathrm{min}$ and PAH at $12 \mathrm{mg} / \mathrm{min}$ for 60 minutes

Inulin at $40-50 \mathrm{mg} / \mathrm{min}$ and $\mathrm{PAH}$ at $10-15 \mathrm{mg} / \mathrm{min}$

at a rate of $0.6 \mathrm{ml} / \mathrm{min}$ for 60 minutes

Inulin $40 \mathrm{ml} 10 \%$ and PAH $10 \mathrm{ml} 20 \%$ in

at a rate of $0.4 \mathrm{ml} / \mathrm{min}$ for $90-120$ minutes

Dextrose $10 \%$ containing $0.5 \mathrm{~g}$ of inulin

at a rate of $8 \mathrm{ml} / \mathrm{minute}$

(to give plateau plasma levels of about $30 \mathrm{mg}$ inulin/100ml)

not stated

Inulin $40 \mathrm{mg}$ and PAH $16 \mathrm{mg}$

at a rate of $8 \mathrm{ml} / \mathrm{min}$ for 60 minutes

Dextrose $5 \%$ and inulin $0.5 \mathrm{~g} / 100 \mathrm{ml}$

at a rate of $8 \mathrm{ml} / \mathrm{min}$ (such that a constant plasma concentration of about $30 \mathrm{mg} / 100 \mathrm{ml}$ was achieved)

Inulin $60 \mathrm{ml}$ and PAH $29 \mathrm{ml}$ in $180 \mathrm{ml} \mathrm{NaCl}$

at a rate of $0.83 \mathrm{ml} / \mathrm{min}$ during 60 minutes

Dextran $264 \mathrm{ml}$, Inulin $75 \mathrm{ml}$ and PAH $36 \mathrm{ml}$

at a rate of $1 \mathrm{ml} / \mathrm{min}$ for 60 minutes

Dextran 10\%, PAH 2\% and Inulin 5\%

at a rate of $1 \mathrm{ml} / \mathrm{min}$ for 60 minutes

Dextran $175 \mathrm{ml}, \mathrm{PAH} 24 \mathrm{ml}$ and Inulin $50 \mathrm{ml}$

at a rate of $1 \mathrm{ml} / \mathrm{min}$ for 60 minutes

Inulin $30 \mathrm{mg} / \mathrm{kg}$ for 60 minutes

Inulin $70 \mathrm{ml}$ and $340 \mathrm{ml} \mathrm{NaCl}$

at a rate of $1 \mathrm{ml} / \mathrm{min}$ for 60 minutes

PAH $9.6 \mathrm{~g}$, Inulin $22.5 \mathrm{~g}$ and $500 \mathrm{ml} 0.9 \% \mathrm{NaCl}$

at a rate of $1 \mathrm{ml} / \mathrm{min}$ for 60 minutes 


\begin{tabular}{|c|c|c|}
\hline Study & Collection period & Creatinine clearance $(\mathrm{ml} / \mathrm{min})$ measurement methods \\
\hline Ahmed 2009 & $24 h$ & $\operatorname{Ucr}[\mathrm{mg} / \mathrm{dL}] \times \mathrm{Uv}[\mathrm{ml}] / \mathrm{Scr} \times 1140 \mathrm{~min})$ \\
\hline Akram 1982 & $24 h$ & Ucr $[\mathrm{mg} / 100 \mathrm{ml}] \times$ Uv [ml/min] / Scr [mg/100ml] \\
\hline Barden 1996 & $24 h$ & not stated \\
\hline Barron 1995 & $24 h$ & not stated \\
\hline Buttermann 1958 & $24 h$ & not stated \\
\hline Davison 1974 & $24 h$ & not stated \\
\hline Davison 1981 & $24 h$ & not stated \\
\hline Davison 1980 & $24 h$ & not stated \\
\hline Lohsiriwat 2008 & $24 h$ & $\operatorname{Ucr}[\mathrm{mg} / \mathrm{dl}] \times \operatorname{Uv}[\mathrm{ml} / \mathrm{min}] / \mathrm{Scr}[\mathrm{mg} / \mathrm{dL}]$ \\
\hline Mozdzien 1995 & $24 h$ & Ucr $\times$ Uv / Scr \\
\hline Saxena 2012 & $24 h$ & Ucr $[\mathrm{mg} / \mathrm{dL}] \times$ Uv [ml/day] / Scr [mg/dL] x $1440 \mathrm{~min} /$ day \\
\hline Schneider 1996 & $24 h$ & not stated \\
\hline Vural 1998 & $24 h$ & not stated \\
\hline Wang 1994 & $24 h$ & not stated \\
\hline Wright 1987 & $2 \mathrm{~h}$ & Ucr x Uv / Scr \\
\hline Yilmaz 2016 & $24 \mathrm{~h}$ & not stated \\
\hline
\end{tabular}

Table 5 Description of glomerular filtration rate measurement methods for creatinine clearance. $\mathrm{UV}=$ urine volume, $\mathrm{Ucr}=$ creatinine measured in urine, $\mathrm{Scr}=$ creatinine measured in serum blood 


\begin{tabular}{|c|c|}
\hline Study & Measurement method \\
\hline Abdul-Karim 1978 & Modified Jaffé reaction using Lloyd's reagent \\
\hline Ahmed 2009 & Alkaline pircrate reactions with a DAX96 (Bayer) machine \\
\hline Akram 1982 & Alkaline picrate method (Jaffe's Reaction) \\
\hline Chapman 1998 & Jaffé reaction and a Beckman 2 auto-analyser \\
\hline Davison 1981 & Enzymatic method using an LKB reaction rate analyser \\
\hline Kristensen 2007 & $\begin{array}{l}\text { Creatininase enzymatic method on a Hitachi Modular P analysis system with } \\
\text { reagent }\end{array}$ \\
\hline Lohsiriwat 2008 & $\begin{array}{l}\text { Colorimetric method based on Jaffés reaction by an automatic analyser } \\
\text { Hitachi } 705\end{array}$ \\
\hline Milne 2002 & not stated \\
\hline Mozdzien 1995 & Jaffé reaction by an automatic analyser Hitachi \\
\hline Roberts 1996 & not stated \\
\hline Saxena 2012 & not stated \\
\hline Strevens 2002 & $\begin{array}{l}\text { Kodak Ektachem } 700 \text { XR-C system using the enzymes creatinine } \\
\text { amidohydrolase and creatinine amidinohydrolase }\end{array}$ \\
\hline Vural 1998 & Jaffé method \\
\hline Yilmaz 2016 & not stated \\
\hline
\end{tabular}

Table 6 Description of measurement methods used in studies with serum creatinine. 
Study Participation Adequate description of participants' characteristics

\begin{tabular}{|c|c|}
\hline & Parity or gravidity \\
\hline & Health or comorbidities of participants \\
\hline & Clear reporting of weeks amenorrhea \\
\hline & Ethnicity \\
\hline & Height \\
\hline & Non-pregnant weight/BMl \\
\hline & Use of medication or supplements \\
\hline & Adequate description of participant recruitment \\
\hline & Adequate description of inclusion and exclusion criteria \\
\hline \multirow[t]{2}{*}{ Study Attrition } & Reasons for loss to follow-up/drop-out are provided \\
\hline & $\begin{array}{l}\text { Adequate description of participants lost to follow-up / differences } \\
\text { between participants who completed and drop-outs }\end{array}$ \\
\hline \multirow{2}{*}{$\begin{array}{l}\text { Variable } \\
\text { Measurements }\end{array}$} & Method of measurement is adequately valid and reliable \\
\hline & $\begin{array}{l}\text { The methods and setting are the same for all study participants and } \\
\text { throughout follow up }\end{array}$ \\
\hline Data Reporting & Time frame of measurements (gestational age) are reported as mean \\
\hline \multirow[t]{5}{*}{ Study Design } & Study used a longitudinal study design \\
\hline & $\begin{array}{l}\text { Multiple }(>2) \text { longitudinal pregnant measurements during pregnancy of the } \\
\text { variable }\end{array}$ \\
\hline & Baseline value was a prepregnant measurement of the variable \\
\hline & Score (\%) \\
\hline & Quality \\
\hline
\end{tabular}

Table 7a Quality assessment of 15 included studies based in the QUIPS criteria. The individual studies are defined as a high quality study (HQ), moderate quality study (MQ) or low quality study (LQ). 


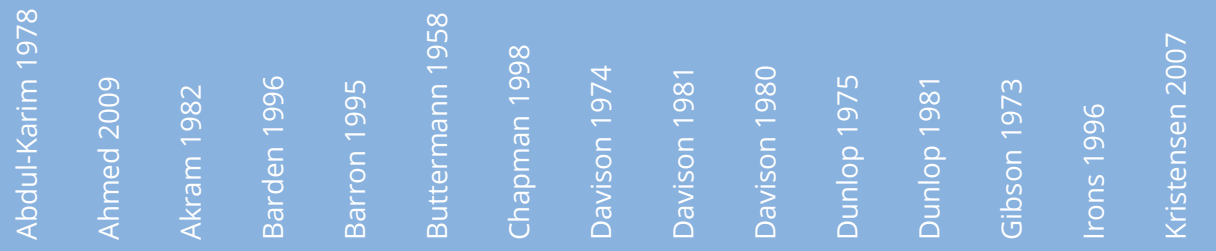

\begin{tabular}{|c|c|c|c|c|c|c|c|c|c|c|c|c|c|c|}
\hline+ & + & - & + & + & + & + & + & + & - & + & + & + & + & + \\
\hline- & + & + & - & - & - & - & - & - & - & - & - & - & - & - \\
\hline - & + & - & - & - & - & - & + & + & + & - & + & + & - & - \\
\hline- & + & - & - & - & - & - & + & + & + & - & + & + & - & + \\
\hline- & - & - & + & + & - & + & + & + & + & - & - & + & + & + \\
\hline- & + & - & + & + & - & + & + & + & + & - & - & + & + & + \\
\hline$?$ & $?$ & $?$ & $?$ & $?$ & $?$ & + & + & + & + & + & + & + & + & + \\
\hline- & + & - & - & + & + & - & + & - & - & + & + & + & + & + \\
\hline+ & + & + & + & + & + & + & + & + & + & + & + & + & + & + \\
\hline- & + & - & + & + & - & - & - & - & - & - & - & - & - & - \\
\hline- & + & - & - & - & - & + & + & + & + & + & + & + & + & - \\
\hline- & - & - & - & - & - & + & + & - & - & - & + & - & - & - \\
\hline
\end{tabular}


Domain

Study Participation

Study Attrition

Variable Measurements

Data Reporting Study Design
Items for consideration

Adequate description of participants' characteristics

Parity or gravidity

Health or comorbidities of participants

Clear reporting of weeks amenorrhea

Ethnicity

Height

Non-pregnant weight/BMl

Use of medication or supplements

Adequate description of participant recruitment

Adequate description of inclusion and exclusion criteria

Reasons for loss to follow-up/drop-out are provided

Adequate description of participants lost to follow-up / differences between participants who completed and drop-outs

Method of measurement is adequately valid and reliable

The methods and setting are the same for all study participants and throughout follow up

Time frame of measurements (gestational age) are reported as mean

Study used a longitudinal study design

Multiple (>2) longitudinal pregnant measurements during pregnancy of the variable

Baseline value was a prepregnant measurement of the variable

Score (\%)

Quality

Table 7b Quality assessment of 14 included studies based in the QUIPS criteria. The individual studies are defined as a high quality study (HQ), moderate quality study (MQ) or low quality study (LQ). 


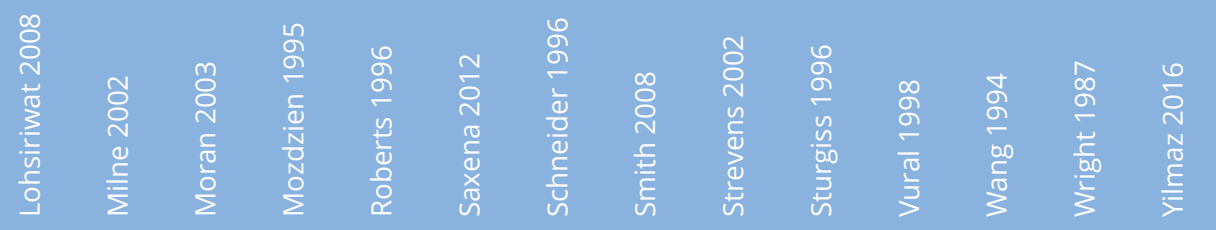

\begin{tabular}{|c|c|c|c|c|c|c|c|c|c|c|c|c|c|}
\hline- & + & - & + & + & + & + & - & - & - & + & - & + & + \\
\hline+ & + & + & + & + & + & - & + & + & + & + & - & + & + \\
\hline
\end{tabular}

\begin{tabular}{|c|c|c|c|c|c|c|c|c|c|c|c|c|c|}
\hline+ & + & + & - & + & + & - & - & - & - & - & - & - & - \\
\hline+ & - & + & - & - & + & + & - & - & + & + & - & - & - \\
\hline+ & + & + & - & + & - & - & + & + & + & + & - & - & + \\
\hline$?$ & $?$ & - & - & $?$ & + & $?$ & - & $?$ & $?$ & $?$ & $?$ & + & $?$ \\
\hline ? & $?$ & - & - & $?$ & - & $?$ & - & $?$ & $?$ & $?$ & $?$ & + & ? \\
\hline
\end{tabular}

\begin{tabular}{|c|c|c|c|c|c|c|c|c|c|c|c|c|c|}
\hline- & + & + & - & + & + & - & + & + & + & - & - & - & - \\
\hline+ & + & + & + & + & + & + & + & + & + & + & + & + & + \\
\hline- & - & - & - & + & + & + & - & + & + & + & + & + & + \\
\hline+ & + & + & + & + & + & - & + & - & + & - & - & + & - \\
\hline- & - & - & + & - & - & - & - & - & - & - & - & + & - \\
\hline - & - & - & - & - & - & - & - & - & - & - & - & - & - \\
\hline 53 & 53 & 47 & 47 & 65 & 71 & 29 & 41 & 47 & 65 & 47 & 24 & 59 & 41 \\
\hline$M Q$ & $M Q$ & $M Q$ & $M Q$ & $H Q$ & $\mathrm{HQ}$ & LQ & $M Q$ & $M Q$ & $H Q$ & $M Q$ & LQ & $M Q$ & $M Q$ \\
\hline
\end{tabular}




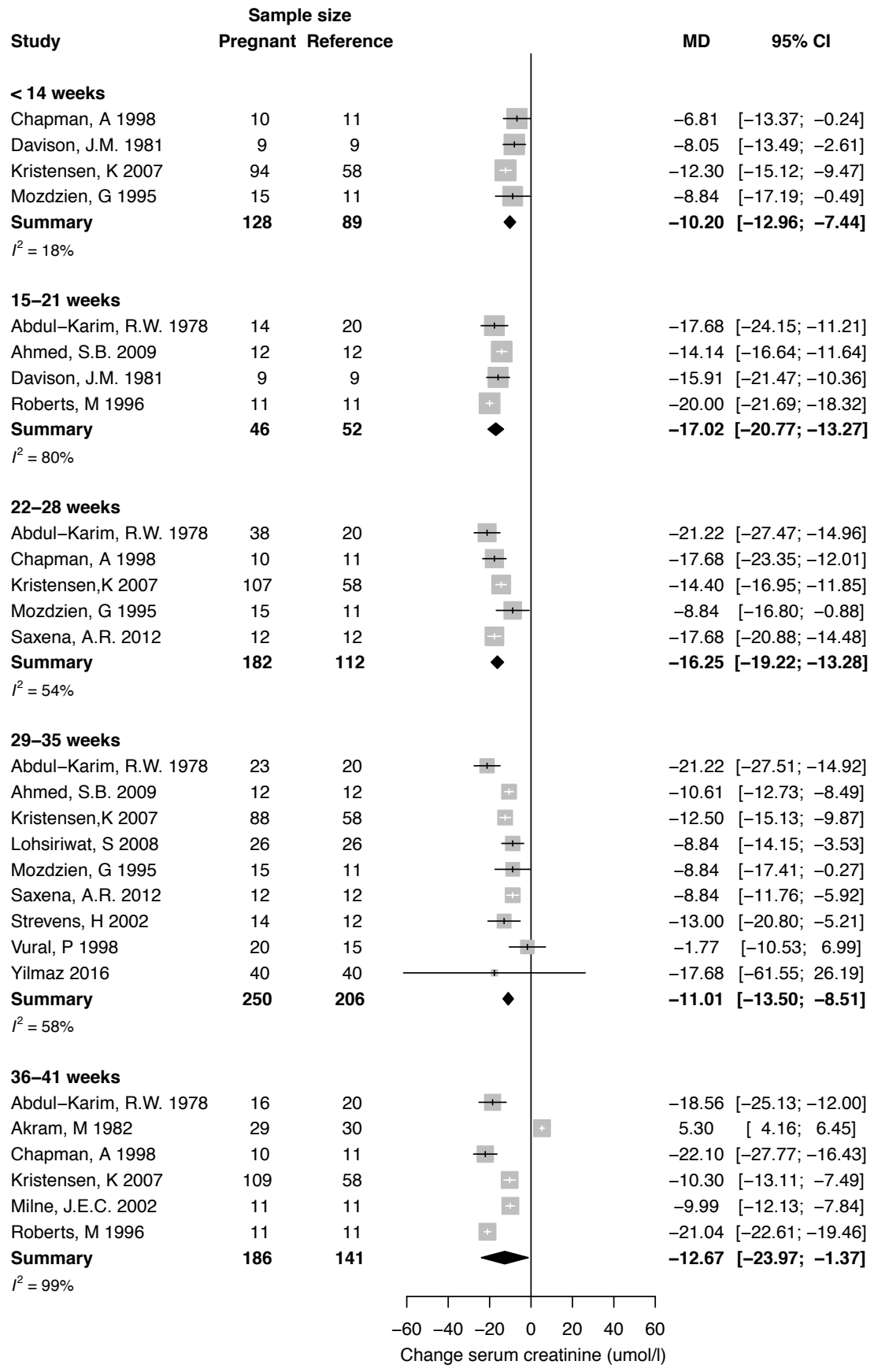

Supplemental file Serum creatinine, in umol/L, during physiologic pregnancies at < 14 weeks, 15 - 21 weeks, 22 - 28 weeks, 29 - 35 weeks, and 36 - 41 weeks' gestation compared to reference values. Serum creatinine is reported as mean difference (MD) between pregnant and reference values with a $95 \% \mathrm{Cl}$. 


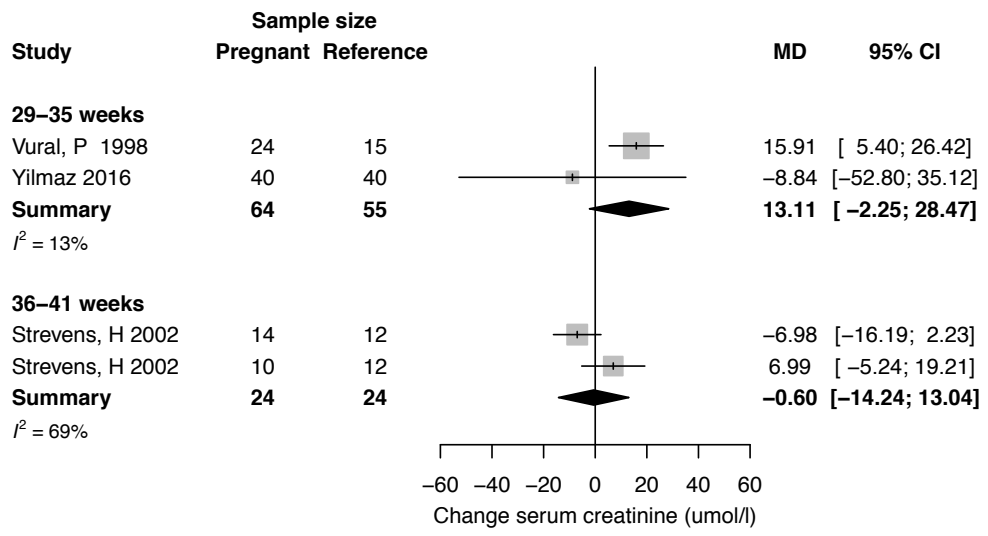

Supplemental file Serum creatinine, in umol/L, during hypertensive complicated pregnancies at 29 - 35 weeks and 36 - 41 weeks' gestation, compared to the reference value. Serum creatinine is reported as mean difference (MD) between pregnant and reference values with a 95\% Cl.

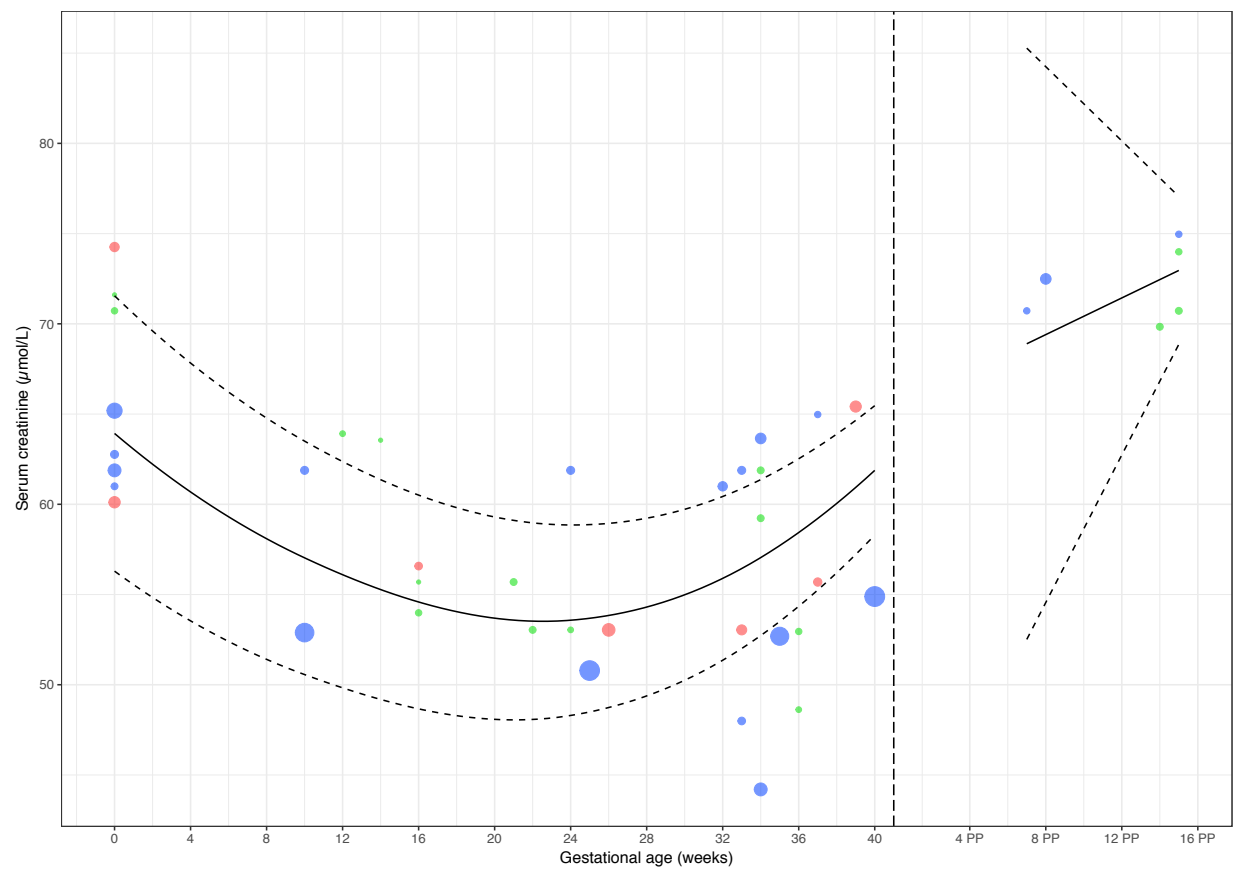

Supplemental file Decrease in serum creatinine, in umol/L, during physiologic pregnancy. The circle size indicates the sample size of the point estimate. The colour of the circle refers to the quality assigned to the study: red $=L Q$, green $=M Q$, blue $=H Q$. Curve fit is weighted by the inverse variance and plotted with $5^{\text {th }}$ and $95^{\text {th }}$ percentiles. The $50^{\text {th }}$ percentile is represented by a solid line. 
d

4

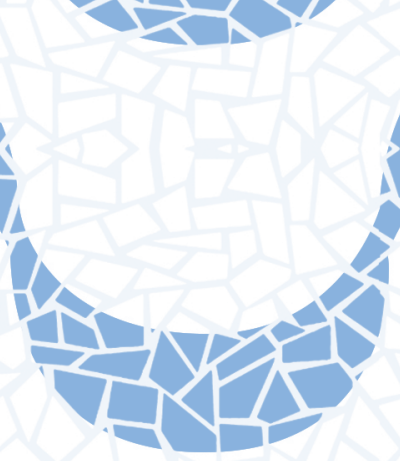

40.2
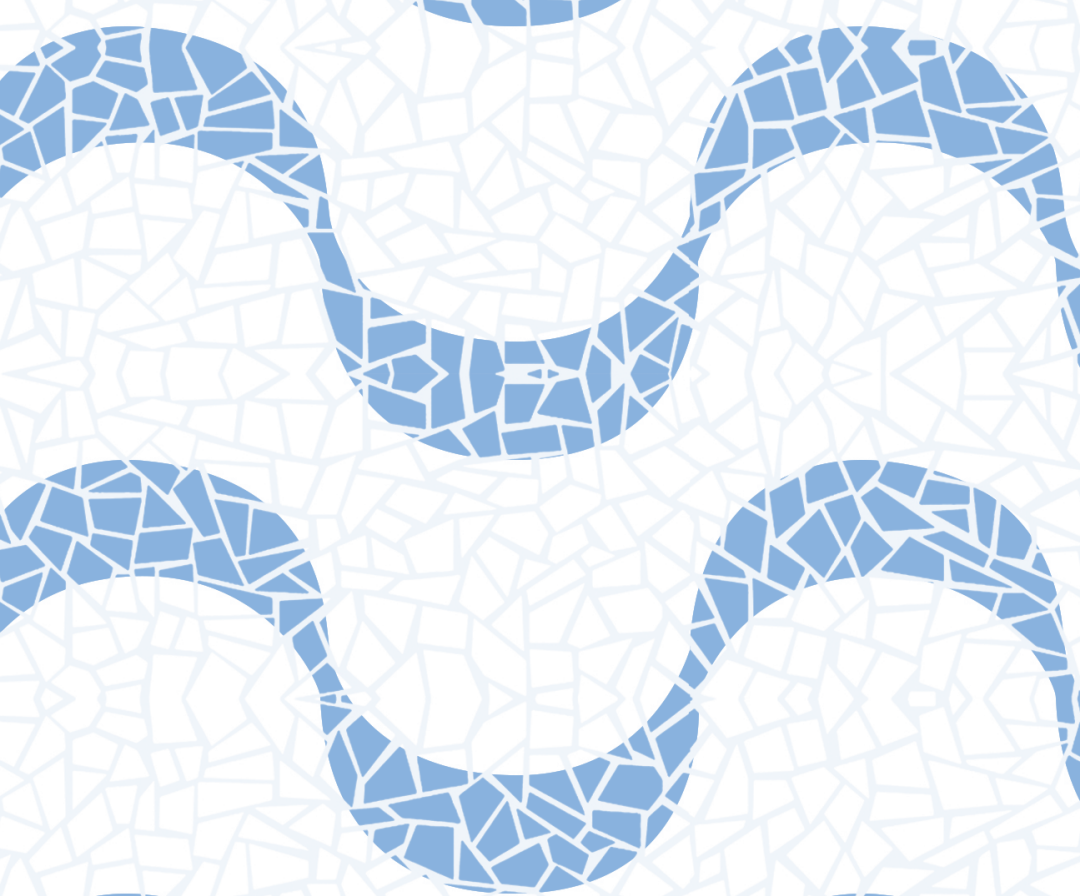

$4 \leq 1$

पर्थ

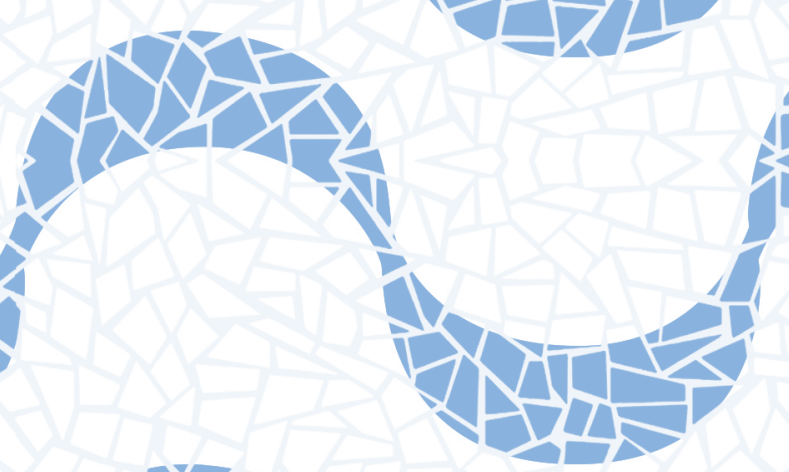

करो
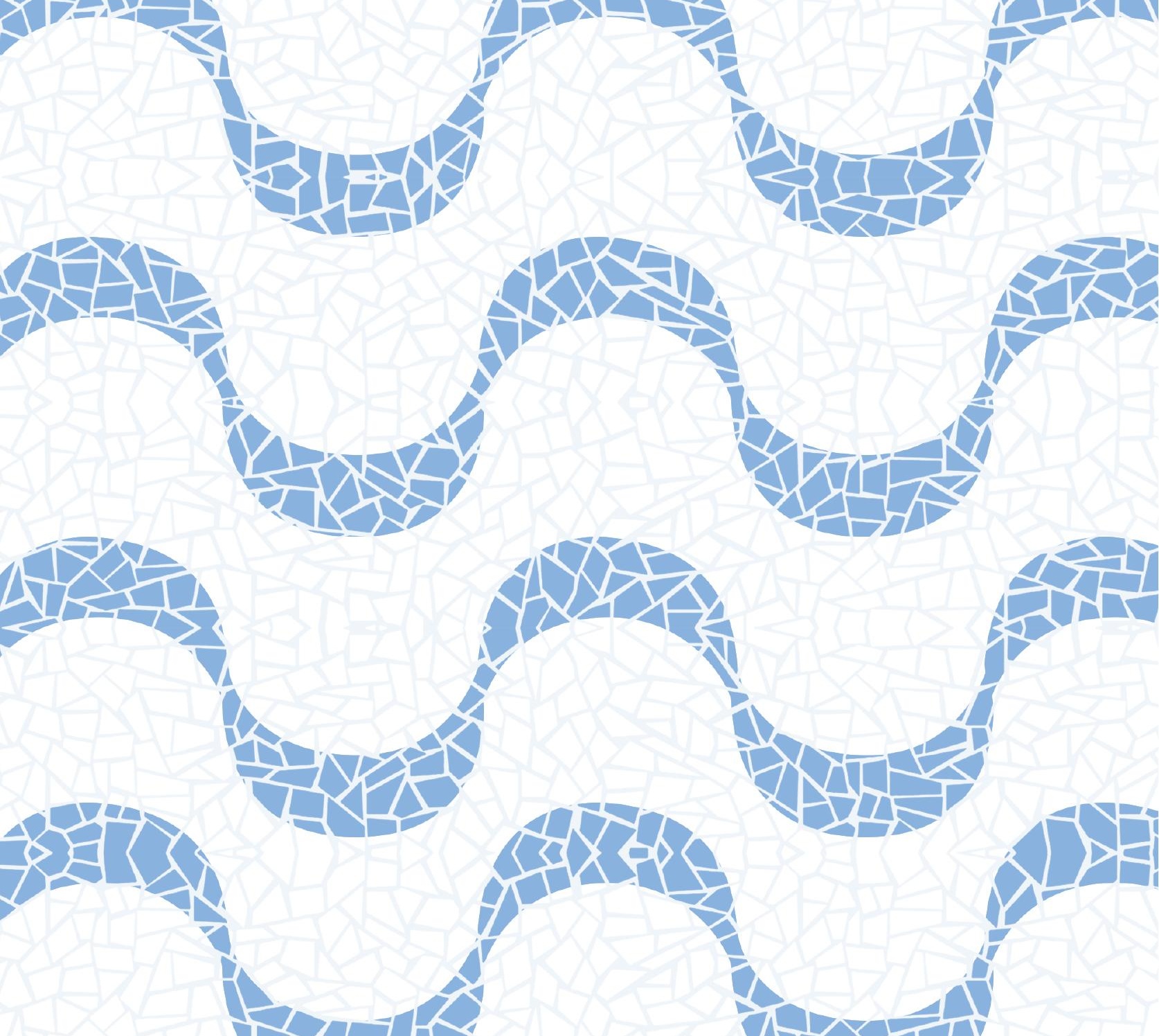


\title{
CHAPTER 4
}

\section{Early pregnancy circulatory adaptation and recurrent hypertensive disease: an explorative study}

\author{
Veronica A. Lopes van Balen \\ Julia J. Spaan \\ Chahinda Ghossein-Doha \\ Sander M.J. van Kuijk \\ Marc E.A. Spaanderman \\ Louis L.H. Peeters
}

Reproductive Sciences; 2013 September 20(9):1069-74 


\section{ABSTRACT}

INTRODUCTION Hypertensive pregnancy disorders are assumed to be preceded by defective spiral artery remodelling. Whether this localized aberration at the implantation site affects the initial maternal systemic cardiovascular and renal adaptation to pregnancy is unclear. We explored, in a high-risk population, whether the initial systemic maternal adaptation to pregnancy differs between women who do and do not develop a recurrent hypertensive disorder later on in pregnancy.

METHODS We enrolled 61 normotensive women with a previous hypertensive disorder of pregnancy and subdivided them in 2 subgroups, based on whether or not their next pregnancy remained uneventful $(n=33)$ or became complicated by a recurrent hypertensive disorder $(n=28)$. We measured before pregnancy and again at $18 \pm 2$ weeks of gestation cardiac output, blood pressure, plasma volume, creatinine clearance, and calculated total peripheral vascular resistance from cardiac output and blood pressure.

RESULTS Both subgroups responded to pregnancy with an increase in cardiac output, plasma volume, heart rate and creatinine clearance, and a decrease in blood pressure and total peripheral vascular resistance. Women who developed a recurrent hypertensive disorder differed from their counterparts with an uneventful next pregnancy by smaller pregnancy-induced increases in creatinine clearance (19\% vs. $31 \%, p=0.035)$ and cardiac output ( $10 \%$ vs. $20 \%, p=0.035)$, respectively.

CONCLUSION The initial systemic cardiovascular and renal adaptations to pregnancy in women who develop a recurrent gestational hypertensive disorder differ from those in their counterparts with an uneventful next pregnancy by smaller rises in creatinine clearance and cardiac output. 


\section{INTRODUCTION}

Hypertensive disorders of pregnancy are a major cause of maternal and perinatal mortality or morbidity. Gestational hypertension is the most common hypertensive disorder during pregnancy with a prevalence of 5 to $18 \%$. Preeclampsia on the other hand is clinically the most relevant gestational hypertensive complication. It has a prevalence of 2 to $5 \%$ in nulliparous women and is responsible for $12 \%$ of maternal deaths in the world ${ }^{1,2}$. The majority of women will have a following pregnancy. During that next pregnancy these women are at increased risk to develop gestational hypertension, preeclampsia, preterm birth and foetal growth restriction ${ }^{3-6}$.

Pregnancy induces marked changes in the cardiovascular and renal systems. A primary fall in the vascular resistance constitutes one of the earliest physiological changes in response to pregnancy ${ }^{7}$. It initiates a chain of events resulting in haemodilution, renal hyperfiltration, rise in cardiac output (CO), higher vascular compliance and a rise in plasma volume (PV), so as to maintain a high-flow and low-resistance circulation ${ }^{8,9}$. At present it is unknown whether this initial adaptive response to pregnancy differs between women with a history of a hypertensive pregnancy complication, who do or do not develop a recurrent hypertensive disorder in their next ongoing pregnancy ${ }^{10-14}$.

The present study was designed to compare the initial systemic hemodynamic and renal adaptations to pregnancy between those who did and those who did not develop a recurrent hypertensive pregnancy disorder in their next pregnancy. To this end, we studied the hemodynamic and renal functions in 61 women with a history of a gestational hypertensive disorder, before and again at $18 \pm 2$ weeks' pregnancy. 


\section{METHODS}

\section{STUDY POPULATION}

This observational cohort study was conducted at Maastricht University Medical Centre in the Netherlands between 2001 and 2011. The study was approved by the institutional medical ethics committee (MEC 10-4-049). We studied renal and hemodynamic function during the first half of pregnancy in women with a history of a gestational hypertensive disorder. Most of these women had a history of severe preeclampsia. Of a total of eightythree women, eleven women did not have a subsequent pregnancy. We excluded women with pre-existent hypertension, diabetes mellitus and renal disease $(n=11)$. All former patients who gave birth before the 34th pregnancy week in the prior pregnancy used 80 mg/day of acetylsalicylic acid from 12 until 36 weeks of pregnancy. In all, 33 (54\%) of the remaining 61 women had a normal next pregnancy whereas 28 (46\%) developed a recurrent gestational hypertensive disorder in their next pregnancy. All women had a preconceptional evaluation (at least 6 months after their previous delivery) and again at $18 \pm 2$ week gestational age. We defined a recurrent hypertensive complication as the development of gestational hypertension, preeclampsia, the haemolysis elevated liver enzymes and low platelet count syndrome (HELLP) syndrome, or placental insufficiency giving rise to a growth restricted infant ${ }^{15}$. According to the guidelines of the National Working Group on High Blood Pressure in Pregnancy preeclampsia was defined as de novo hypertension (arterial blood pressure > 140/90 mmHg, occurring after the 20th week of pregnancy) accompanied by proteinuria (> $300 \mathrm{mg} / 24 \mathrm{~h}$ or $>30 \mathrm{mg} / \mathrm{mmol}$ creatinine). We defined gestational hypertension as de novo hypertension without concomitant proteinuria. The HELLP syndrome was defined as the concomitant development of haemolysis, elevated liver enzymes and a low platelet count. Low birth weight was defined as a birth weight below the 10th centile based on reference curves derived from the Dutch perinatal registration ${ }^{16}$. We defined an uneventful pregnancy as a singleton pregnancy without complications, resulting in the term birth ( $\geq 37$ weeks) of an infant with a birth weight above the $10^{\text {th }}$ centile ${ }^{15}$.

\section{MEASUREMENT}

Measurements were performed in standardized environmental conditions with external disturbances kept to a minimum. Blood pressure was recorded with the subject in left-lateral tilt position. We registered blood pressure every three minutes, using a semiautomatic oscillometric device (Dinamap Vital Signs Monitor 1846; Critikon, Tampa, Florida, USA and Accutorr 2008; Mindray, Medical Netherlands, Hoevelaken) during 30 minutes. The mean arterial pressure (MAP, $\mathrm{mmHg}$ ) was defined as the blood pressure that coincided with the mean of the two highest amplitudes of the pulse detected with the oscillometric device ${ }^{17}$. Laboratory measurements were performed at the laboratory of University 
Medical Centre Maastricht. We obtained the glomerular filtration rate (GFR) by dividing the 24 hours urine creatinine excretion by the concomitant serum creatinine level ${ }^{18}$. We performed cardiac ultrasound measurement using a phased-array echocardiographic Doppler system, a Philips IE 33 system (Philips Medical System, Best, the Netherlands) according to the guidelines of the American Society of Echocardiography ${ }^{19}$. From an apical approach, aortic flow across the aortic valve was measured using continuous wave Doppler ${ }^{20}$. Stroke volume (SV, $\mathrm{mL}$ ) was calculated by multiplying aortic velocity time integral (VTI) with the cross-sectional area measured at the level of the aortic annulus in the parasternal long axis view. The mean VTI, used to calculate SV, was measured by averaging the outer edge tracings of 3 to 5 consecutive CW Doppler recordings ${ }^{20}$. CO (L/min) was obtained by multiplying SV with heart rate (HR, beats/min). The HR was obtained by taking the reciprocal of the mean of 5 consecutive R-R intervals on the electrocardiogram. Peripheral vascular resistance ( $\mathrm{mmHg} / \mathrm{L} / \mathrm{min}$ ) was calculated by the formula: 80 * (MAP/CO). All data were stored on disk and calculations were performed offline using specific software (Excelera, Philips, the Netherlands) by a single observer. We used an indicator dilution technique to estimate plasma volume ( $P V, \mathrm{~mL}$ ) using 3 consecutively obtained venous blood samples taken within 10 min after the administration of a 10\% dextran-70 solution, as specified previously ${ }^{21}$. The total PV was calculated using linear regression analysis. We did not index $\mathrm{CO}$ and PV for body surface area as the standard formulas required for this are based on data from non-pregnant individuals and do not take into account the changes in maternal dimensions during pregnancy 22.

\section{STATISTICAL ANALYSIS}

We used the unpaired t-test to test for differences between groups and Pearson's chisquare test for categorical variables. The paired t-test was used to test for differences over time within groups. We assessed differences present at the pre-pregnancy state, and differences present at the visit closest to 18 weeks of gestation. To assess the differences in the adaptation during the first half of gestation, we computed the change between the pre-pregnancy state and the visit closest to 18 weeks, and tested whether these changes differ between the two groups.

There were some missing values present on the variables of interest. We chose not to impute these missing values, because we only computed pairwise comparisons, and performed no multivariable modelling. The proportions of cases dropped from pairwise comparisons are, in our sample, relatively low (30\%). Data are expressed as group means \pm standard deviation, means $\pm 95 \% \mathrm{Cl}$ or medians and interquartile ranges. A p-value less than, or equal to, 0.05 was considered to indicate a statistical significant difference. All analyses were performed using SPSS version 17.0, property of IBM. 


\section{RESULTS}

Table 1 illustrates the patient selection. Table 2 lists the patient characteristics of the prior and subsequent pregnancies. Both subgroups were comparable with respect to age and BMI, however, prior neonatal birth weight was lower and the gestational age at birth was earlier in women with a recurrent hypertensive complication compared to the uneventful subgroup. Of the 61 women with a history of a hypertensive disorder during pregnancy, 33 (54\%) had an uneventful next pregnancy outcome and 28 (46\%) had their next pregnancy complicated by a recurrent gestational hypertensive disorder. The subsequent pregnancy was complicated by preeclampsia in $29 \%$, by gestational hypertension in 50\%, by foetal growth restriction without hypertension in $14 \%$ and by HELLP syndrome in $7 \%$ of all cases.

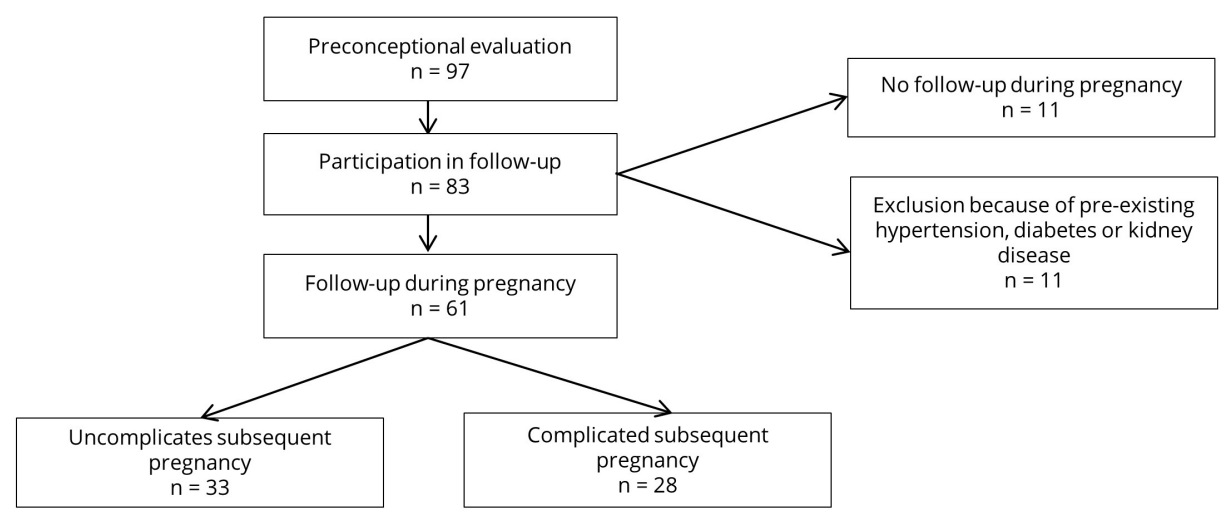

Table 1 Patient selection.

Table 3 lists the adaptive response to pregnancy at $18 \pm 2$ weeks' gestational age in both subgroups. The percent changes of the presumably most relevant variables are illustrated in figure 1. All changes in response to pregnancy in both subgroups were significant. Both subgroups responded to pregnancy with a rise in $\mathrm{CO}$, creatinine clearance, $\mathrm{PV}$, and a fall in MAP and peripheral vascular resistance. Women in the recurrent subgroup tended to have a lower PV (in $\mathrm{mL}$ ) prior to pregnancy than their counterparts in the uneventful subgroup. The magnitude of the changes in response to pregnancy was comparable in both subgroups, except for creatinine clearance and cardiac output, which was smaller in the recurrence subgroup than in the uneventful subgroup, $34 \mathrm{ml} / \mathrm{min}$ (23 - $45 \mathrm{ml} / \mathrm{min}$ ) vs. $19 \mathrm{ml} / \mathrm{min}(8-29 \mathrm{ml} / \mathrm{min})$ and $0.9 \mathrm{~L} / \mathrm{min}(0.6-1.3 \mathrm{ml} / \mathrm{min}) \mathrm{vs} .0 .4 \mathrm{~L} / \mathrm{min}(0.1-0.8 \mathrm{ml} /$ min), respectively. 


\begin{tabular}{|llll|}
\hline Prior pregnancy & $\begin{array}{l}\text { Uncomplicated } \\
\text { subsequent } \\
\text { pregnancy } \\
n=33\end{array}$ & $\begin{array}{l}\text { Complicated } \\
\text { subsequent } \\
\text { pregnancy } \\
n=28\end{array}$ & p-value \\
\hline GH & $2(6 \%)$ & $1(3 \%)$ & \\
\hline PE & $6(18 \%)$ & $10(36 \%)$ & \\
\hline PE/HELLP & $25(76 \%)$ & $17(61 \%)$ & 0.902 \\
\hline Gestational age at delivery (days) & $212 \pm 25$ & $213 \pm 25$ & 0.763 \\
\hline Birth weight (gram) & $1192 \pm 677$ & $1138 \pm 696$ & 0.210 \\
\hline Birth weight (centile) & p20-p50 & p20-p50 & \\
\hline Subsequent pregnancy & & & 0.644 \\
\hline Pre-pregnancy BMI (kg/m²) & $26 \pm 6$ & $26 \pm 5$ & \\
\hline GH & & $14(50,0 \%)$ & \\
\hline PE/HELLP & & $8(28.6 \%)$ & 0.435 \\
\hline HELLP & & $2(7.1 \%)$ & $0.012^{*}$ \\
\hline Growth restriction & $30 \pm 4$ & $30 \pm 4$ & $0.028^{*}$ \\
\hline Age (years) & $273 \pm 9$ & $261 \pm 26$ & 0.327 \\
\hline Gestational age at delivery (days) & $3392 \pm 441$ & $2684 \pm 1115$ & \\
\hline Birth weight (gram) & p20-p50 & $<p 10$ & \\
\hline Birth weight (centile) & & & \\
\hline
\end{tabular}

Table 2 Patient characteristic of prior and subsequent pregnancies.

An unpaired t-test was used to analyse differences between groups and a Pearson's chi-square test for categorical variables. GH = gestational hypertension, $\mathrm{PE}=$ pre-eclampsia, HELLP = haemolysis elevated liver enzymes and low platelet count, GA = gestational age. Data are adapted and represented as means and standard deviation and as frequencies with percentages (\%) for categorical variables, p-values < 0, 05 are considered significant. An asterisk represents a p-value less than 0.05 .

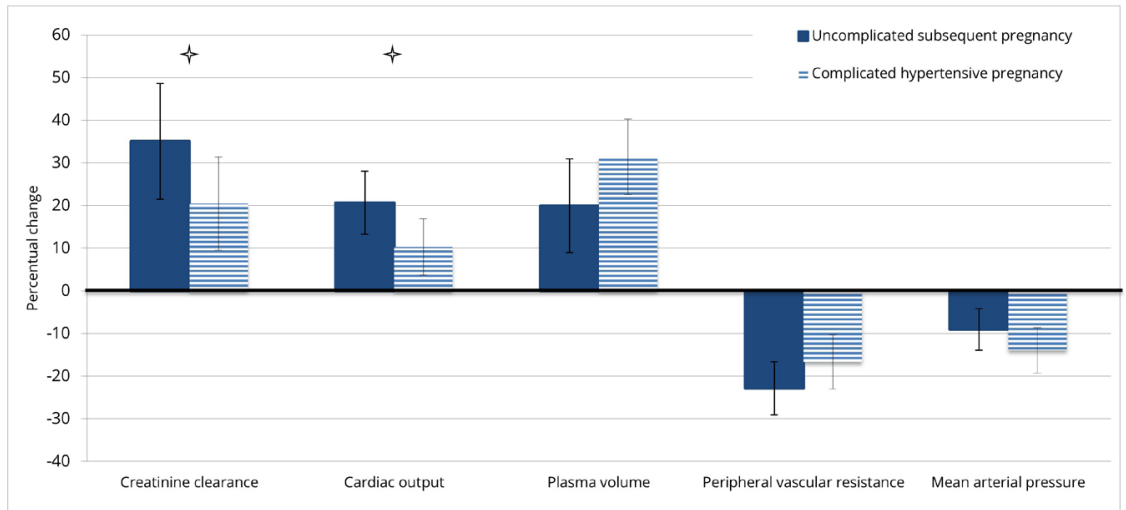

Figure 1 Adaptation to pregnancy in recurrent gestational hypertensive disorder and an uncomplicated subsequent pregnancy, percentual change. Bars represent mean change (\%) with 95\% confidence interval between measurement before pregnancy and at $18 \pm 2$ weeks' pregnancy. $p<0.05$ is considered to indicate a significant difference and are represented by a star above the bar chart. 
Creatinine clearance $(\mathrm{mL} / \mathrm{min})$ $\mathrm{n}=47$

Cardiac output (L/min)

$\mathrm{n}=50$

Plasma volume (ml)

$n=42$ n

Pre-pregnancy

$18 \pm 2$ weeks pregnancy

$\triangle$

$\mathrm{n}$

Pre-pregnancy

$18 \pm 2$ weeks pregnancy

$\triangle$

n

Pre-pregnancy

$18 \pm 2$ weeks pregnancy

$\triangle$

$n$

Pre-pregnancy

$18 \pm 2$ weeks pregnancy

$\triangle$

n

Pre-pregnancy

$18 \pm 2$ weeks pregnancy

$\triangle$

Table 3 Adaptation to pregnancy in recurrent hypertensive pregnancy and uncomplicated subsequent pregnancy. A paired t-test was used to test for differences over time within groups. Data are given as mean with 95\% confidence interval. $\Delta=$ difference between pre-pregnancy and $18 \pm 2$ weeks' gestational age. An asterisk represents a p-value less than 0.05 . 


\begin{tabular}{lll} 
Uncomplicated subsequent pregnancy & Recurrent hypertensive pregnancy & p-value \\
25 & 22 & \\
$109(101-117)$ & $109(98-120)$ & 0.985 \\
$144(134-154)$ & $131(119-142)$ & 0.085 \\
$34(23-45)$ & $19(8-29)$ & $0.035^{\star}$ \\
\hline 27 & 23 & 0.199 \\
$4.9(4.6-5.2)$ & $5.2(4.8-5.5)$ & 0.143 \\
$5.9(2.6-6.3)$ & $5.6(5.3-5.9)$ & $0.035^{\star}$ \\
$0.9(0.6-1.3)$ & $0.4(0.1-0.8)$ & \\
24 & 18 & 0.065 \\
$2403(2210-2596)$ & $2144(1973-2316)$ & 0.125 \\
$2813(2634-2992)$ & $2621(2453-2789)$ & 0.310 \\
$152(-78-381)$ & $309(105-514)$ & 0.814 \\
27 & 23 & 0.065 \\
$1515(1415-1615)$ & $1499(1403-1595)$ & 0.237 \\
$1138(1064-1212)$ & $1227(1167-1288)$ & \\
$-379(-497--261)$ & $-282(-399-164)$ & 0.447 \\
12 & 14 & 0.671 \\
$92(87-96)$ & $94(89-99)$ & 0.168 \\
\hline $81(76-86)$ & $81(78-87)$ & \\
$-9(-13--4)$ & $-15(-22--7)$ & \\
\hline
\end{tabular}




\section{DISCUSSION}

In this study we found that, in women with a hypertensive complicated pregnancy in their history, who developed a recurrent hypertensive disorder in their next pregnancy, the initial maternal renal and hemodynamic adaptive response to pregnancy was attenuated when compared to that in their counterparts who had an uneventful subsequent pregnancy. The attenuated response consisted of a smaller rise in creatinine clearance and cardiac output.

To our knowledge, an attenuated increase in creatinine clearance preceding clinical symptoms has not been described before. The marked rise in creatinine clearance in normal pregnancy is probably secondary to the up to $60 \%$ rise in renal blood flow ${ }^{23}$. The resulting renal hyperfiltration represents the largest measurable gestational change in a non-uterine organ function. Therefore, differences in the adaptive response to pregnancy of women with recurrent hypertensive disease are probably easiest discernible on the basis of this particular phenomenon. It is conceivable that altered set-points in the volume homeostasis ${ }^{24}$ in women with a subnormal pre-pregnant PV are responsible for a lower rise in pregnancy-induced renal blood flow ${ }^{25}$ and with it, creatinine clearance. If not all nephrons are functional, single nephron perfusion may still be raised, but probably to a lesser degree ${ }^{26,27}$.

Most mammals including humans, respond to pregnancy with a fall in systemic vascular resistance ${ }^{28}$. The resulting relative arterial underfill leads to activation of the baroreceptor and with it, a compensatory increase in $\mathrm{CO}^{7,24}$ along PV expansion by means of stimulation of renal sodium and water retention ${ }^{28,29}$. During a gestational hypertensive complication the systemic vascular resistance is raised ${ }^{30}$. The pattern of change in peripheral vascular resistance in the period preceding a gestational hypertensive disorder is still unclear ${ }^{31-33}$ Our explorative study supports the findings of Rang et el. ${ }^{31}$ that at $18 \pm 2$ weeks' gestation, the peripheral vascular resistance is probably already raised in the recurrent subgroup relative to that in the uncomplicated subgroup. We were unable to discern a difference in MAP in early pregnancy.

The pattern of change in MAP with advancing pregnancy in a low-risk population supports a higher MAP prior to a symptomatic hypertensive pregnancy disorder ${ }^{32-34}$. In this context, we want to emphasize that our observations in a high-risk population may not be typical for the events reported to occur in a low-risk population. 
To our surprise pre-pregnant PV in the recurrence subgroup was not lower than in the subgroup of women with a normal pregnancy course. Others found evidence for a subnormal plasma volume to predispose to recurrent preeclampsia ${ }^{35-39}$. This unexpected observation brings up the limitations of this clinical study. Firstly, the study population was both heterogeneous and modest in size. These features reduce the power of the study. Secondly, the relatively wide spectrum of outcome measures will have magnified the inter- and intra-patient variability in various measurements. Thirdly, in this descriptive study we could not standardize clinical management during the pregnancy used for group selection. Finally, outcome variables such as creatinine clearance, CO and PV are composite variables, which carry a relatively large coefficient of variation, which may even be larger during pregnancy ${ }^{18,40}$. It follows that these preliminary results require confirmation in a larger study population.

\section{PERSPECTIVES}

Seemingly healthy women who experienced a hypertensive complication in their first pregnancy are generally considered to be at high risk for recurrence. Therefore, they are subjected to intensive surveillance in their next pregnancy. However, only $7 \%$ of them are destined to develop a severe recurrent hypertensive complication in their next pregnancy ${ }^{41}$. So far, there are no simple and sensitive screening methods to identify the subgroup of really high-risk women early in the next pregnancy. This study provides evidence for an attenuated initial renal and cardiovascular adaptive response to pregnancy in the subgroup of women developing a recurrent - also mild - hypertensive disorder in their next pregnancy, relative to those who had an uneventful next pregnancy. Although the methodologic limitations reduced the discretionary potential of this study, we do not expect that these differences are sufficiently sensitive to be used in a screening test. Nevertheless, they support the concept that in former patients, even a mild recurrent hypertensive complication developing in the next pregnancy is preceded by an abnormal initial renal and cardiovascular adaptive response to pregnancy. 


\section{REFERENCES}

1. WHO. The World health report: make every mother and child count. 2005.

2. NVOG. Guidelines; Hypertensive diseases. 2005.

3. Dildy GA, 3rd, Belfort MA, Smulian JC. Preeclampsia recurrence and prevention. Semin. Perinatol. Jun 2007;31(3):135-141.

4. Langenveld J, Buttinger A, van der Post J, Wolf H, Mol BW, Ganzevoort W. Recurrence risk and prediction of a delivery under 34 weeks of gestation after a history of a severe hypertensive disorder. Br. J. Obstet. Gynaecol. Apr 2011;118(5):589-595.

5. van Rijn BB, Hoeks LB, Bots ML, Franx A, Bruinse HW. Outcomes of subsequent pregnancy after first pregnancy with early-onset preeclampsia. Am. J. Obstet. Gynecol. Sep 2006;195(3):723-728.

6. Hernandez-Diaz S, Toh S, Cnattingius S. Risk of pre-eclampsia in first and subsequent pregnancies: prospective cohort study. Br. Med. J. 2009;338:2255.

7. Conrad KP. Mechanisms of Renal Vasodilatation and Hyperfiltration During Pregnancy: Current Perspectives and Potentia; Implications for Preeclampsia. Endothelium. 2005 2005;12:57-62.

8. Duvekot JJ, Cheriex EC, Pieters FA, Menheere PP, Peeters LL. Early pregnancy changes in hemodynamics and volume homeostasis are consecutive adjustments triggered by a primary fall in systemic vascular tone. Am. J. Obstet. Gynecol. Dec 1993;169(6):1382-1392.

9. Duvekot JJ, Peeters LLH. Maternal cardiovascular hemodynamic adaptation to pregnancy. Obstet. Gynecol. Surv. Dec 1994;49(12 Suppl):S1-14.

10. Duvekot JJ, Cheriex EC, Pieters FA, Peeters LLH. Severely impaired fetal growth is preceded by materna hemodynamic maladaptation in very early pregnancy. Acta Obstet. Gynecol. Scand. Oct 1995;74(9):693-697.

11. Steegers EA, von Dadelszen P, Duvekot JJ, Pijnenborg R. Pre-eclampsia. Lancet. Aug 21 2010;376(9741):631-644.

12. Pijnenborg R, Luyten C, Vercruysse L, Van Assche FA. Attachment and differentiation in vitro of trophoblast from normal and preeclamptic human placentas. Am. J. Obstet. Gynecol. Jul 1996;175(1):30-36.

13. Pijnenborg R, Vercruysse $L$, Hanssens $M$. The uterine spiral arteries in human pregnancy: facts and controversies. Placenta. Sep-Oct 2006;27(9-10):939-958.

14. Noris MP, N and Remuzzi, G. Mechanisms of disease; pre-eclampsia. Nature Clinical Practice; Nephrology. 2005;1(2).

15. Report of the National High Blood Pressure Education Program Working Group on High Blood Pressure in Pregnancy. Am. J. Obstet. Gynecol. 2000;183(1):s1-s22.

16. Visser GH, Eilers PH, Elferink-Stinkens PM, Merkus HM, Wit JM. New Dutch reference curves for birthweight by gestational age. Early Hum. Dev. Dec 2009;85(12):737-744.

17. Inc C. Dinamap Adult/Pediatric and Neonatal Vital Signs Monitor Model 1864 Operation Manual. 1984.

18. Koetje PM, Spaan JJ, Kooman JP, Spaanderman MEA, Peeters LLH. Pregnancy reduces the accuracy of the estimated glomerular filtration rate based on Cockroft-Gault and MDRD formulas. Reprod. Sci. May 2011;18(5):456-462.

19. Picard MH, Adams D, Bierig SM, et al. American Society of Echocardiography recommendations for quality echocardiography laboratory operations. Journal of the American Society of Echocardiography : official publication of the American Society of Echocardiography. Jan 2011;24(1):1-10.

20. Sahn DJ DA, Kisslo J, Weyman A. Recommendations regarding quantitation in M-mode echocardiography: results of a survey of echocardiographic measurements. Circulation. 1978; 58(6):1072-1083.

21. van Kreel BK, van Beek E, Spaanderman ME, Peeters LL. A new method for plasma volume measurements with unlabeled dextran-70 instead of 125I-labeled albumin as an indicator. Clin. Chim. Acta. Jul 6 1998;275(1):71-80.

22. van Oppen AC, van der Tweel I, Duvekot JJ, Bruinse HW. Use of cardiac index in pregnancy: is it justified? Am. J. Obstet. Gynecol. Sep 1995;173(3 Pt 1):923-928.

23. Heineman MJ. Obstetrie en Gynaecologie De voortplanting van de mens 2007.

24. Schrier RW, Durr JA. Pregnancy: an overfill or underfill state. American journal of kidney diseases : the official journal of the National Kidney Foundation. Apr 1987;9(4):284-289.

25. Jeyabalan A, Conrad KP. Renal function during normal pregnancy and preeclampsia. Front. Biosci. 2007;12:24252437.

26. Lafayette RA, Druzin M, Sibley R, et al. Nature of glomerular dysfunction in pre-eclampsia. Kidney Int. Oct 1998;54(4):1240-1249.

27. Lafayette R. The kidney in preeclampsia. Kidney Int. Mar 2005;67(3):1194-1203. 
28. Duvekot JJ, Peeters LLH. Renal hemodynamics and volume homeostasis in pregnancy. Obstet. Gynecol. Surv. Dec 1994;49(12):830-839.

29. Schrier RW, Briner VA. Peripheral arterial vasodilation hypothesis of sodium and water retention in pregnancy: implications for pathogenesis of preeclampsia-eclampsia. Obstet. Gynecol. Apr 1991;77(4):632-639.

30. Lindheimer MD. Hypertension in pregnancy. Hypertension. Jul 1993;22(1):127-137.

31. Rang S, van Montfrans GA, Wolf H. Serial hemodynamic measurement in normal pregnancy, preeclampsia, and intrauterine growth restriction. Am. J. Obstet. Gynecol. May 2008;198(5):519 e511-519.

32. Easterling TR, Benedetti TJ, Schmucker BC, Millard SP. Maternal hemodynamics in normal and preeclamptic pregnancies: a longitudinal study. Obstet. Gynecol. Dec 1990;76(6):1061-1069.

33. Bosio PM, McKenna PJ, Conroy R, O'Herlihy C. Maternal central hemodynamics in hypertensive disorders of pregnancy. Obstet. Gynecol. Dec 1999;94(6):978-984.

34. De Paco C, Kametas N, Rencoret G, Strobl I, Nicolaides KH. Maternal cardiac output between 11 and 13 weeks of gestation in the prediction of preeclampsia and small for gestational age. Obstet. Gynecol. Feb 2008;111(2 Pt 1):292-300.

35. Andrietti S, Kruse AJ, Bekkers SC, Sep S, Spaanderman M, Peeters LL. Cardiac adaptation to pregnancy in women with a history of preeclampsia and a subnormal plasma volume. Reprod. Sci. Dec 2008;15(10):10591065.

36. Spaanderman MEA, Ekhart T, van Eyck J, de Leeuw P, Peeters LLH. Preeclampsia and maladaptation to pregnancy: a role for atrial natriuretic peptide? Kidney Int. Oct 2001;60(4):1397-1406.

37. Aardenburg R, Spaanderman MEA, Ekhart TH, van Eijndhoven HW, van der Heijden OW, Peeters LLH. Low plasma volume following pregnancy complicated by pre-eclampsia predisposes for hypertensive disease in a next pregnancy. Br. J. Obstet. Gynaecol. Nov 2003;110(11):1001-1006.

38. Bernstein IM, Ziegler W, Badger GJ. Plasma volume expansion in early pregnancy. Obstet. Gynecol. May 2001;97(5 Pt 1):669-672.

39. Aardenburg R, Spaanderman MEA, Courtar DA, van Eijndhoven HW, de Leeuw PW, Peeters LLH. A subnormal plasma volume in formerly preeclamptic women is associated with a low venous capacitance. J. Soc. Gynecol. Investig. Feb 2005;12(2):107-111.

40. Alper AB, Yi Y, Webber LS, et al. Estimation of glomerular filtration rate in preeclamptic patients. Am. J. Perinatol. Nov 2007;24(10):569-574.

41. van Kuijk SM, Nijdam ME, Janssen KJ, et al. A model for preconceptional prediction of recurrent early-onset preeclampsia: derivation and internal validation. Reprod. Sci. Nov 2011;18(11):1154-1159. 


\title{
CHAPTER 5
}

\section{Prevalence of chronic kidney disease after preeclampsia}

\author{
Veronica A. Lopes van Balen \\ Julia J. Spaan \\ Tom Cornelis \\ Marc E.A. Spaanderman
}

Journal of Nephrology; 2016 June 30(3):403-409 


\section{ABSTRACT}

INTRODUCTION Preeclampsia (PE), an endothelial disease that affects kidney function during pregnancy, is correlated to an increased future risk of cardiovascular and chronic kidney disease. The Kidney Disease Improving Global Outcomes (KDIGO) 2012 guideline emphasizes the combined role of glomerular filtration rate (GFR) and albuminuria in determining the frequency of monitoring of kidney function. In this study we determined the prevalence of CKD in women with a history of PE. We investigated how many seemingly healthy women would need monitoring of kidney function according to the KDIGO guideline.

METHODS We included 775 primiparous women with a history of PE. They were at least four months postpartum, had no pre-existing hypertension, diabetes or kidney disease. We estimated GFR by the CKD-EPI-equation and urinary albumin loss by albumin creatinine ratio in a 24 hour urine collection.

RESULTS Most women, 669 (86.3\%), had a normal GFR and absent albuminuria. According to the KDIGO guideline $13.7 \%$ women would require at least yearly monitoring of kidney function. Only $1.4 \%$ was classified to be at high risk for kidney function deterioration.

CONCLUSION Monitoring of kidney function seems relevant for about one in seven women with a history of preeclampsia women, mainly due to albuminuria. Albuminuria should be evaluated postpartum to identify those women that need further monitoring of kidney function. 


\section{INTRODUCTION}

Preeclampsia (PE), an endothelial disease characterized by hypertension and commonly coincided by proteinuria, is the most common cause of maternal death in the Netherlands. Maternal death due to PE occurs in 3.5 of 100.000 live births, accounting for $39 \%$ of total maternal death. PE affects kidney function during pregnancy and also increases the risk of future chronic hypertension, chronic kidney disease (CKD) and cardiovascular disease 1-6. PE is associated with a fourfold increased risk of developing end stage kidney disease within 10 years after pregnancy. Having more than one preeclamptic pregnancy, a lowbirth weight offspring or a preterm delivery increases this risk even further ${ }^{3}$. Glomerular endotheliosis observed during a preeclamptic pregnancy may be fully reversible when it does not reach the level of glomerular scarring ${ }^{7}$. The reversibility is obviously dependent on cessation of the cause of endothelial injury. In case of PE this is effectuated by termination of pregnancy and delivery of the placenta. It can take months before the fibrinous and granular material deposits in the glomerulus fully disappear ${ }^{8}$.

Data on follow-up of kidney function of these women are scarce. Some case-control and cohort studies suggest that most women with a history of PE do maintain good kidney function after PE 9-11. However, there remains a group of women that will have decreased glomerular filtration rate (GFR) and/or persistent urinary protein loss. The existence of moderately increased albuminuria, at any glomerular filtration rate, is considered an independent risk factor for the development of cardiovascular disease 12-14. The Kidney Disease Improving Global Outcomes (KDIGO) 2012 criteria emphasize the independent role of GFR and albuminuria to assess the prognosis of chronic kidney disease (CKD). The guideline provides us with comprehensive evidence based-decisions in order to monitor patients with chronic kidney disease. In this study we determined the prevalence of CKD in a large cohort of women with a history of PE. We aimed to establish how many of these seemingly healthy former patients would qualify for further monitoring of kidney function according to the KDIGO guideline. 


\section{METHODS}

In Maastricht University Medical Centre a postpartum cardiovascular screening is offered to women with a history of PE. The aim of this screening is to determine underlying disorders that might have contributed to the development of PE and to advise them on future pregnancies and cardiovascular implications. All women with a history of a vascular complicated pregnancy (either maternal hypertensive or foetal growth restricted) were offered a postpartum cardiovascular screening after delivery in our hospital; some were also referred from other hospitals in the country, between 1996 and 2014. For our analysis, to improve homogeneity of the studied population, we only selected women with PE (gestational hypertensive with de novo proteinuria) that entered our program ${ }^{15}$. We excluded multiparous and women who were evaluated less than 4 months after delivery. We also excluded women that were diagnosed with hypertension, diabetes mellitus or kidney disease prior to the complicated pregnancy. All measurements were performed in our assessment unit under controlled circumstances.

\section{MEASUREMENTS}

Measurements during the postpartum cardiovascular screening were performed in standardized environmental conditions. Blood pressure was recorded for a period of 30 minutes at 3 minute intervals using a semiautomatic oscillometric device in half-sitting position. Median values of 9 subsequent recordings were used for analysis. An extensive (family) history was taken. Urine analysis was done to determine albumin loss on a urine sample collected during 24 hours. Albuminuria was measured by immunochemical analysis. We categorized women into normal to mild albuminuria $<3 \mathrm{mg} / \mathrm{mmol}$, moderately increased albuminuria 3 - $30 \mathrm{mg} / \mathrm{mmol}$, and severely increased albuminuria $>30 \mathrm{mg} / \mathrm{mmol}$ 16. We also collected blood samples for serum creatinine, which was obtained by the Jaffe method until 2012 and thereafter through an enzymatic method (all three measurements with Cobas 8000 modular analyser series [Roche Diagnostics, Basel, Switzerland]). We estimated GFR by the CKD-EPI-equation in $\mathrm{ml} / \mathrm{min} / 1.73 \mathrm{~m}^{2}$. Both formulas are dependent on serum creatinine and used for female and Caucasian. When serum creatinine was below or equal to $0.7 \mathrm{mg} / \mathrm{dl}$, we used $144 \times$ (serum creatinine $/ 0.7)^{\wedge-0.329} \times 0.993^{\wedge a g e}$. When serum creatinine was above $0.7 \mathrm{mg} / \mathrm{dl}$, we used $144 \times($ serum creatinine / 0.7)^-1.209 $\times 0.993$ ^age. Albuminuria was quantified by albumin creatinine ratio (ACR). 
KDIGO

The KDIGO guideline provides a table with recommendations on frequency of monitoring of kidney function based on the risk of progression of CKD and another table on monitoring by a general clinician or referral to a nephrologist. We combined both tables into one overview table (Table 3). In this table the yellow colour indicates a preferred frequency of monitoring once a year, whereas orange indicates monitoring twice a year, light red three times a year, and dark red four times a year or more. Referral for monitoring of kidney function by a more specialized care, preferably a nephrologist, is indicated for severely increased albuminuria (ACR $>3 \mathrm{mg} / \mathrm{mmol}$ ) or decreased GFR $\left(<30 \mathrm{ml} / \mathrm{min} / 1.73 \mathrm{~m}^{2}\right)$, according to the KDIGO guideline, as is shown.

The KDIGO table on cardiovascular mortality is based on a meta-analysis ${ }^{16}$ of 105,872 people, extracted from 14 different studies (see Table 5). To calculate the adjusted relative risk of cardiovascular mortality for our study group, we classified all women based on this table.

\section{STATISTICAL ANALYSIS}

All analyses were performed using SPSS version 21.0, property of IBM. Data were expressed as group means and standard deviation or medians and interquartile ranges. A $p$-value $<0.05$ was considered to indicate a statistical significant difference. To analyse the differences between groups with normally distributed data we used the unpaired t-test. For non-normal distributed data, we used the Mann-Whitney $U$ test. Dichotomous data was analysed with a Chi square test. We corrected for months postpartum, age, gestational onset of hypertensive complication and gestational age at delivery where appropriate. 


\section{RESULTS}

Women with a history of PE, evaluated at least 4 months postpartum, were selected after exclusion of women that were multiparous or had pre-existing hypertension, diabetes mellitus and/or kidney disease (Table 1). Data required for categorization of kidney function were incomplete in $3.1 \%$ of all cases, leaving 775 complete cases.

Table 2 shows the characteristics of our study group. Women were on average 10 months postpartum, normotensive, had an early onset PE ( $<34$ weeks' gestation) and gave birth prematurely. The baseline values were equal for those women excluded due to missing urinary analyses. Women with persistent moderately to severely increased albuminuria (ACR $>3 \mathrm{mg} / \mathrm{mmol}$ ) were younger, gave birth at an earlier gestational age to smaller children and had higher blood pressure compared to women with normal to mildly increased albuminuria (ACR $<3 \mathrm{mg} / \mathrm{mmol}$ ). They had an average amount of protein loss of $196 \mathrm{mg} / 24$ hours IQR [154 - 237]) and used significantly more antihypertensive drugs, in particular renin- angiotensin system (RAS) blocking drugs.

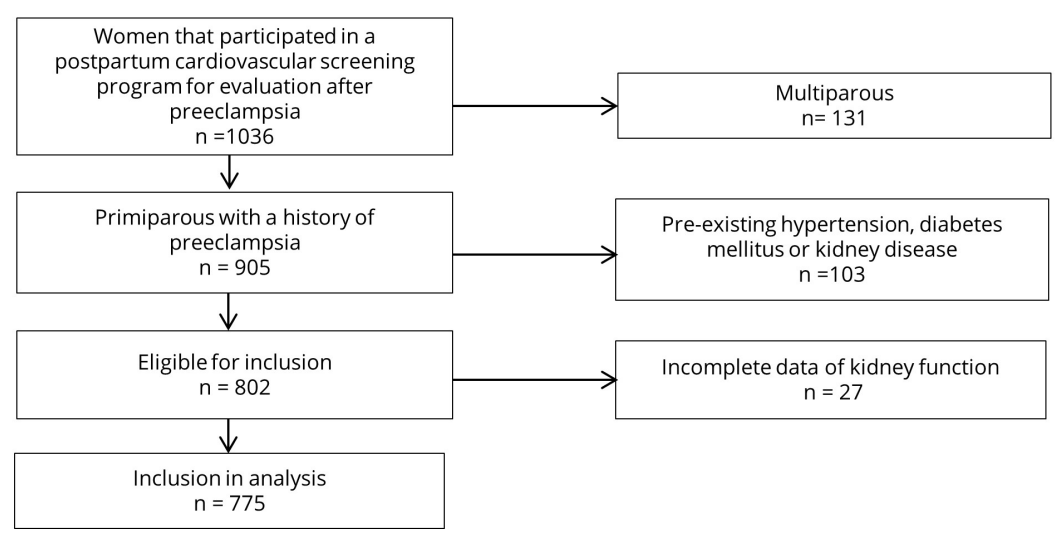

Table 1 Flowchart.

Table 3 shows the KDIGO table on the frequency of monitoring. According to the guideline, for 106 (13.7\%) women with a history of PE, monitoring of kidney function is indicated once yearly (yellow category). This is mostly due to a moderately to severely increased ACR with a normal GFR (> $\left.90 \mathrm{ml} / \mathrm{min} / 1.73 \mathrm{~m}^{2}\right)(11.9 \%)$. Only $1.8 \%$ had a decreased GFR $(<90 \mathrm{ml}$ $\mathrm{min} / 1.73 \mathrm{~m}^{2}$ ) and moderately to severely increased ACR. Moreover, 11 (1.4\%) women would need a more specialized care, preferably by a nephrologist, based on severely increased albuminuria (ACR $>3 \mathrm{mg} / \mathrm{mmol}$ ). 


\begin{tabular}{|c|c|c|c|c|}
\hline & $\begin{array}{l}\text { All } \\
\text { participants } \\
n=775\end{array}$ & $\begin{array}{l}\text { ACR }<3 \\
\mathrm{mg} / \mathrm{mmol} \\
\mathrm{n}=669\end{array}$ & $\begin{array}{l}\text { ACR } \geq 3 \\
\mathrm{mg} / \mathrm{mmol} \\
\mathrm{n}=106\end{array}$ & p-value \\
\hline Age (years) & $31 \pm 4.2$ & $31 \pm 4.1$ & $30 \pm 4.5$ & $<0.001$ * \\
\hline Months postpartum & $10[6-18]$ & $12[7-19]$ & $10[5-10]$ & 0.945 \\
\hline $\mathrm{BMI}\left(\mathrm{kg} / \mathrm{m}^{2}\right)$ & $25.0 \pm 4.8$ & $25.0 \pm 4.6$ & $25.0 \pm 5.2$ & 0.872 \\
\hline Smoking & $109(14 \%)$ & $94(14 \%)$ & $15(14 \%)$ & 0.978 \\
\hline Gestational age at diagnosis (weeks) & $31^{+5} \pm 4^{+1}$ & $31^{+6} \pm 4^{+1}$ & $30^{+6} \pm 4^{+0}$ & $0.049 *$ \\
\hline Gestational age at delivery (weeks) & $33^{+3} \pm 3^{+5}$ & $33^{+4} \pm 3^{+5}$ & $32^{+5} \pm 3^{+6}$ & $0.012^{*}$ \\
\hline Birth weight (gram) & $\begin{array}{l}1856 \\
{[1188-2447]}\end{array}$ & $\begin{array}{l}1890 \\
{[1230-2450]}\end{array}$ & $\begin{array}{l}1657 \\
{[980-2266]}\end{array}$ & $0.010 *$ \\
\hline Birth weight percentile & $20[9-40]$ & $20[9-45]$ & 18 [6-36] & $0.037 *$ \\
\hline Systolic blood pressure $(\mathrm{mmHg}) \dagger$ & $116 \pm 13$ & $116 \pm 12$ & $120 \pm 12$ & $<0.001 *$ \\
\hline Diastolic blood pressure $(\mathrm{mmHg}) \dagger$ & $72 \pm 9$ & $71 \pm 9$ & $75 \pm 10$ & $<0.001$ * \\
\hline $\begin{array}{l}\text { Glomerular filtration rate } \\
\left(\mathrm{ml} / \mathrm{min} / 1.73 \mathrm{~m}^{2}\right) \dagger\end{array}$ & $105 \pm 15$ & $105 \pm 15$ & $107 \pm 16$ & 0.471 \\
\hline Antihypertensive drugs & $66(8.5 \%)$ & $52(7.7 \%)$ & $14(13.2 \%)$ & $0.031 *$ \\
\hline ACR (mg/mmol) & $0.9[0.5-1.9]$ & $0.8[0.5-1.3]$ & $5[4-10]$ & \\
\hline RAS blocking drugs & $26(3.4 \%)$ & $16(2.4 \%)$ & $10(9.4 \%)$ & $<0.001^{*}$ \\
\hline
\end{tabular}

Table 2 Characteristics of all primiparous women with a history of preeclampsia and stratified by albumin creatinine ratio (ACR). RAS $=$ renin angiotensin system. $*=p<0,05, t=$ adjusted for gestational age at delivery and age. Data were expressed as group means and standard deviation or median and interquartile range.

Table 4 details the postpartum renal albumin loss categorized by time interval. Moderately to increased albumin loss was most frequent within the first months after delivery and seems to be less prominent in women who were more than $1 \frac{1}{2}$ years postpartum. Women who were two years postpartum had an increased ACR in $6 \%$ of the cases.

Table 5 presents the KDIGO table on calculated cardiovascular mortality risk for our study group. It shows that about $25 \%$ of women have a relative risk of 0.9 of cardiovascular mortality, 22\% has a 1.3 relative risk, and $10 \%$ has a 1.5 relative risk. Some women (7.6\%) even have a 2.3 increased risk. The median adjusted relative risk of cardiovascular mortality was $1.25 \pm 0.6$ (not shown in figure). The increased cardiovascular mortality risk is mostly based on increased albumin loss as opposed to a reduced GFR. 


\begin{tabular}{|c|c|c|c|}
\hline$n=775$ & $A C R<3$ & ACR 3 - 30 & $A C R>30$ \\
\hline GFR > 89 & $573(73.9 \%)$ & $83(10.7 \%)$ & $9(1.2 \%)$ \\
\hline GFR 60 - 89 & $96(12.4 \%)$ & $12(1.6 \%)$ & $1(0.1 \%)$ \\
\hline GFR 45 - 59 & & Monitoring & $1(0.1 \%)$ \\
\hline GFR 30 - 44 & & & \\
\hline GFR 15 - 29 & \multirow{2}{*}{\multicolumn{2}{|c|}{ Referral }} & \\
\hline GFR $<15$ & & & \\
\hline
\end{tabular}

Table 3 KDIGO table on frequency of monitoring showing the prevalence of CKD in primiparous women with a history of PE. Frequency of monitoring based on the risk of progression of CKD depending on ACR and GFR (19). Once a year monitoring is represented by yellow, twice by orange, three times for light red and four times by dark red. GFR is expressed in $\mathrm{ml} / \mathrm{min} / 1.73 \mathrm{~m}^{2}$ and ACR in $\mathrm{mg} / \mathrm{mmol}$. With referral a more specialized care is indicated, preferably by a nephrologist.

\begin{tabular}{llll} 
Months postpartum & $>3$ ACR $\mathrm{mg} / \mathrm{mmol} \mathrm{n} /$ total $(\%)$ & Adjusted odds \\
\hline $4-5$ & $51 / 150$ & $(25 \%)$ & Ref \\
$6-11$ & $33 / 248$ & $(12 \%)$ & $0.43(\mathrm{Cl} 0.26-0.71)^{\star}$ \\
$12-17$ & $13 / 96$ & $(12 \%)$ & $0.44(\mathrm{Cl} 0.23-0.87)^{\star}$ \\
$18-23$ & $1 / 58$ & $(2 \%)$ & $0.06(\mathrm{Cl} 0.00-0.46)^{\star}$ \\
$>24$ & $8 / 117$ & $(6 \%)$ & $0.25(\mathrm{Cl} 0.11-0.57)^{\star}$ \\
\hline
\end{tabular}

Table 4 Albuminuria after preeclampsia categorized by time interval between delivery and screening. Odds ratio represents the risk of persistent albuminuria ( $>3$ ACR) at a certain time period postpartum compared with the reference group (4 - 6 months postpartum), corrected for gestational age at delivery and maternal age. An asterisk represents a p-value less than 0.05.

\begin{tabular}{|c|c|c|c|c|c|c|c|c|}
\hline$n=775$ & \multicolumn{2}{|c|}{$A C R<1$} & \multicolumn{2}{|c|}{ ACR 1 - 2.9} & \multicolumn{2}{|c|}{ ACR $3-29.9$} & \multicolumn{2}{|c|}{$A C R>30$} \\
\hline eGFR > 105 & 0.9 & $193(24.9 \%)$ & 1.3 & $152(19.6 \%)$ & 2.3 & $58(7.5 \%)$ & 2.0 & $9(1.2 \%)$ \\
\hline eGFR 90 - 105 & Ref & $143(18.5 \%)$ & 1.5 & $85(11.0 \%)$ & 1.7 & $25(3.2 \%)$ & 3.7 & - \\
\hline eGFR 75 - 90 & 1.0 & $60(7.7 \%)$ & 1.3 & $24(3.1 \%)$ & 1.6 & $6(0.7 \%)$ & 3.7 & $1(0.1 \%)$ \\
\hline eGFR 60 - 75 & 1.1 & $9(1.2 \%)$ & 1.4 & $3(0.4 \%)$ & 2.0 & $6(0.8 \%)$ & 4.1 & - \\
\hline eGFR $45-60$ & 1.5 & - & 2.2 & - & 2.8 & - & 4.1 & $1(0.1 \%)$ \\
\hline eGFR 30 - 45 & 2.2 & - & 2.7 & - & 3.4 & - & 5.2 & - \\
\hline eGFR 15 - 30 & 1.5 & - & 7.9 & - & 4.8 & - & 8.1 & - \\
\hline
\end{tabular}

Table 5 KDIGO table on cardiovascular mortality risk showing the number of primiparous women with a history of preeclampsia in each risk category. The estimated cardiovascular mortality risk is based on a meta-analysis of 105,872 people. Each cell represents a pooled relative risk from this meta-analysis. Colours reflect the ranking of adjusted relative risk. The point estimates for each cell were ranked from 1 (lowest) to 28 (highest). The categories with rank numbers 1 - 8 are green, rank numbers 9 - 14 are yellow, rank numbers 15 - 21 are orange, and the rank numbers $22-28$ are coloured red (32). Normal to mild albuminuria is further categorized into ACR $<1$ and 1 - 3 mg/ $\mathrm{mmol}$. Number of women in each cell with percentage is given. GFR is expressed in $\mathrm{ml} / \mathrm{min} / 1.73$ $\mathrm{m}^{2}$ and $A C R$ in $\mathrm{mg} / \mathrm{mmol}$. 


\section{DISCUSSION}

Our study indicates that, based on the KDIGO monitoring guideline, about one in seven women after PE should have at least yearly monitoring of their kidney function, mainly due to moderately increased albuminuria. Only $1.4 \%$ was classified to be at high risk for kidney function deterioration and in need of referral to specialist care, preferably a nephrologist. About $6 \%$ of women had an increased ACR two years postpartum.

PE can manifest as a combination of maternal and foetal factors. It can be considered as more than one disease with major differences between near-term mild PE and PE that is associated with low birthweight and preterm delivery ${ }^{17}$. Our data indicate that mainly persistent albuminuria would classify $13.7 \%$ women after PE as having CKD. The prevalence of CKD in the general adult, and usually older, population is approximately $10 \%$ 18. Women with a history of preeclampsia are younger and have a 4 to 5 times increased risk of end stage kidney disease compared to women with no PE in their history. Having more than one preeclamptic pregnancy, a low-birth weight offspring or a preterm delivery increases the risk of CKD even further ${ }^{3}$.

The KDIGO guideline is written for the general population and indicates that kidney disease persisting for more than four months is considered to be chronic, irrespective of the aetiology. This provides us with guidance on how often we should follow women with a history of PE after delivery to ensure that albuminuria will regress over time and to clarify which women should be further investigated for underlying kidney disease. The persistence of albuminuria is considered to be either due to an undiagnosed preexisting kidney disease, a higher susceptibility of these women to develop CKD or due to irreversible kidney damage during the preeclamptic pregnancy. Though it is known that in several cases kidney disease is entirely reversible, either spontaneously or with treatment, it is prudent to ascertain whether this actually happens ${ }^{19}$.

The prevalence of albuminuria in our study population is lower compared with other data that mention $42 \%$ after 3 - 5 years postpartum and 20 - 30\% after 7 years postpartum $4,9,20,21$. On the contrary, some other studies conducted at a later postpartum interval show no increased persistent (micro) albuminuria ${ }^{1,11}$. We did however exclude women with known conditions that affect kidney function. It could be that, due to the heterogeneity of the disease of $\mathrm{PE}$, kidney function is affected differently but also recovers at a different time rate, possibly depending on a woman's general health. Another contributing factor could be the method used for the acquisition of data on albuminuria as some studies use 24 hour and others 8 hour collection or a single urine sample. 
We would also like to address that the risk of cardiovascular mortality is increased in women with a history of PE. As the risk of cardiovascular disease is also increased in women with CKD we could hypothesize that women with a history of PE and CKD have an even more increased risk of cardiovascular disease (CVD) ${ }^{22}$. Appropriate treatment, aimed at preventing progression of CVD, is important since blood pressure control, lifestyle advice and exercise can slow down or even reverse progression of both CVD and CKD ${ }^{23}$. Appropriate blood pressure regulation seems particularly relevant for this group, as in our study women with persistent albuminuria had a higher blood pressure compared to those with normal to mildly increased albuminuria. They did already use significantly more antihypertensive medication, in particular RAS blocking drugs. Blood pressure regulation, as part of cardiovascular risk management, improves the prognosis of CKD ${ }^{24}$. Albuminuria screening does not only seem effective but also cost-effective, when taking possible treatment with ACE inhibitors into account, seeing as it reduces the risk of both CKD and CVD ${ }^{25}$. Interestingly, screening of albuminuria in a high risk population is found to correlate with the occurrence of future renal replacement therapy ${ }^{26}$. This makes screening for albuminuria in a population of women with a history of preeclampsia even more plausible. From a practical point of view we would advise evaluation of kidney function, for women with a history of preeclampsia, at the regular 6 weeks postpartum control. Women who then have microalbuminuria could be seen one year thereafter by their general physician for follow-up of kidney function. If a cardiovascular screening should be available the inclusion of a test for microalbuminuria would be advisable.

There are some shortcomings that need to be addressed that effect extrapolation to other populations. First of all, even though the KDIGO offers a guideline with evidence based-decisions that provide the possibility to take both GFR and ACR into account when evaluating kidney function, it is difficult to extrapolate outcomes and the need for frequent follow-up of young and otherwise healthy women. It is possible that these women have very different relative risk changes and rates of progression than corresponding subjects with other renal diseases like diabetes or primary glomerular disease. The KDIGO guideline does not define patients by aetiology. Secondly, it may be that our population not completely represents the general formerly preeclamptic group of women as our population primarily consists of women with an early onset preeclampsia.

Early onset disease may cause an overestimation of the prevalence of CKD due to the severity of the disease and its impact on the endothelium. On the other hand, the results might be an underestimate of the prevalence of CKD after preeclampsia due to the exclusion of co-morbidities known to be related to kidney dysfunction such as diabetes mellitus, pre-existing hypertension and known kidney disease. 
The cross-sectional nature of this study is also an inevitable shortcoming as is the lack of a control group. Because not all women with a history of preeclampsia that delivered in our hospital partook in the screening and because women were referred from other hospitals we are not able to fully contextualize to the overall PE population. A future longitudinal study would be preferable to ascertain changes over time, preferably including data on pre-pregnancy GFR or albuminuria values to determine a possible pre-existing kidney condition.

The key strengths of this study are the relative long postpartum period, when compared to a regular check-up at 6 weeks after delivery and the large study population. In addition, recent studies indicate that CKD-EPI gives the best estimate of GFR when compared to the gold standard of $\mathrm{I}^{125}$ iothalamate ${ }^{27}$. It has the highest accuracy in women when compared to both the MDRD and Cockcroft-Gault formula and the use of CKD-EPI has, to date, not been reported in women with a history of PE ${ }^{28}$.

\section{CONCLUSION}

Monitoring of kidney function seems relevant for about one in seven formerly preeclamptic women, mainly due to albuminuria. Albuminuria should be evaluated postpartum to identify those women that need further monitoring of kidney function. 


\section{REFERENCES}

1. Spaan JJ, Ekhart T, Spaanderman MEA, Peeters LLH. Remote hemodynamics and renal function in formerly preeclamptic women. Obstetrics and gynecology. Apr 2009;113(4):853-859.

2. Vikse BE. Pre-eclampsia and the risk of kidney disease. The Lancet. 2013;382:104-106.

3. Vikse BE, Irgens LM, Leivestad T, Skjaerven R, Iversen BM. Preeclampsia and the risk of end-stage renal disease. N. Engl. J. Med. Aug 21 2008;359(8):800-809.

4. McDonald S, Han Z, Walsh M, Gerstein H, Devereaux P. Kidney Disease After Preeclampsia: A Systematic Review and Meta-analysis. Am. J. Kidney Dis. 2010;55(6):1026-1039.

5. van der Graaf AM, Toering TJ, Faas MM, Lely AT. From preeclampsia to renal disease: a role of angiogenic factors and the renin-angiotensin aldosterone system? Nephrol. Dial. Transplant. Oct 2012;27 Suppl 3:iii51-57.

6. Bellamy L, Casas JP, Hingorani AD, Williams DJ. Pre-eclampsia and risk of cardiovascular disease and cancer in later life: systematic review and meta-analysis. Br. Med. J. Nov 10 2007;335(7627):974

7. Lafayette R. The kidney in preeclampsia. Kidney Int. Mar 2005;67(3):1194-1203.

8. Hussein W, Lafayette RA. Renal function in normal and disordered pregnancy. Curr. Opin. Nephrol. Hypertens. Jan 2014;23(1):46-53.

9. Durk Berks, Eric A.P. Steegers, Marek Molas, Visser W. Resolution of Hypertension and Proteinuria After Preeclampsia. Obstet. Gynecol. 2009;114(6):1307-1314.

10. Lampinen KH, Ronnback M, Groop PH, Kaaja RJ. Renal and vascular function in women with previous preeclampsia: a comparison of low- and high-degree proteinuria. Kidney Int. Nov 2006;70(10):1818-1822.

11. Sandvik MK, Hallan S, Svarstad E, Vikse BE. Preeclampsia and prevalence of microalbuminuria 10 years later. Clin. J. Am. Soc. Nephrol. Jul 2013;8(7):1126-1134.

12. Chambers JC, Fusi L, Malik IS, Haskard DO, De Swiet M, Kooner JS. Association of maternal endothelia dysfunction with preeclampsia. JAMA. Mar 28 2001;285(12):1607-1612.

13. Arnlov J, Evans JC, Meigs JB, et al. Low-grade albuminuria and incidence of cardiovascular disease events in nonhypertensive and nondiabetic individuals: the Framingham Heart Study. Circulation. Aug 16 2005;112(7):969-975.

14. Schmieder RE, Schrader J, ZidekW, et al. Low-grade albuminuria and cardiovascular risk : what is the evidence? Clin. Res. Cardiol. May 2007;96(5):247-257.

15. Report of the National High Blood Pressure Education Program Working Group on High Blood Pressure in Pregnancy. Am. J. Obstet. Gynecol. 2000;183(1):s1-s22.

16. Levey AS, de Jong PE, Coresh J, et al. The definition, classification, and prognosis of chronic kidney disease: a KDIGO Controversies Conference report. Kidney Int. Jul 2011;80(1):17-28.

17. Sibai B, Dekker G, Kupferminc M. Pre-eclampsia. Lancet. Feb 26-Mar 4 2005;365(9461):785-799.

18. Levey AS, Coresh J. Chronic kidney disease. The Lancet. 2012;379:165-180.

19. Coresh J, Selvin E, Stevens L, et al. Prevalence of Chronic Kidney Disease in the United States. 2007;298(17):20382047.

20. Bar J, Boris Kaplan, Clara Wittenberg, et al. Microalbuminuria after pregnancy complicated by pre-eclampsia. Nephrol. Dial. Transplant. 1999(14):1129-1132.

21. Nisell H, Lintu H, Lunell NO, Mollerstrom G, Pettersson E. Blood pressure and renal function seven years after pregnancy complicated by hypertension. BJOG. Nov 1995;102(11):876-881.

22. Kurth T, de Jong PE, Cook NR, Buring JE, Ridker PM. Kidney function and risk of cardiovascular disease and mortality in women: a prospective cohort study. Br. Med. J. Jun 29 2009;338:b2392.

23. van der Velde M, Matsushita K, Coresh J, et al. Lower estimated glomerular filtration rate and higher albuminuria are associated with all-cause and cardiovascular mortality. A collaborative meta-analysis of high-risk population cohorts. Kidney Int. Jun 2011;79(12):1341-1352.

24. Parati G, Ochoa JE, Bilo G. Blood pressure variability, cardiovascular risk, and risk for renal disease progression. Curr. Hypertens. Rep. Oct 2012;14(5):421-431.

25. Boersma C, Gansevoort R, Pechlivanoglou P, et al. Screen-and-Treat Strategies for Albuminuria to Prevent Cardiovascular and Renal Disease: Cost-Effectiveness of Nationwide and Targeted Interventions Based on Analysis of Cohort Data From the Netherlands. Clin. Ther. 2010;32(6):1103-1121.

26. van der Velde M, Halbesma N, de Charro FT, et al. Screening for albuminuria identifies individuals at increased renal risk. J. Am. Soc. Nephrol. Apr 2009;20(4):852-862. 
27. Chen J, Muntner P, Hamm LL, et al. The metabolic syndrome and chronic kidney disease in U.S. adults. Ann. Intern. Med. Feb 03 2004;140(3):167-174.

28. Michels WM, Grootendorst DC, Verduijn M, Elliott EG, Dekker FW, Krediet RT. Performance of the CockcroftGault, MDRD, and new CKD-EPI formulas in relation to GFR, age, and body size. Clin. J. Am. Soc. Nephrol. Jun 2010;5(6):1003-1009. 


\title{
CHAPTER 6
}

\section{Endothelial and kidney function in women with a history of preeclampsia and healthy parous controls}

\author{
Veronica A. Lopes van Balen \\ Julia J. Spaan \\ Tom Cornelis \\ Wieteke M. Heidema \\ Ralph R. Scholten \\ Marc E.A. Spaanderman
}




\section{ABSTRACT}

INTRODUCTION Preeclampsia (PE) is a pregnancy related endothelial disease characterized by hypertension and albuminuria. Postpartum endothelial dysfunction often persists in these women after PE. We postulate that in women with a history of PE reduced endothelial dependent vasodilation coincides with attenuated kidney function, as both reflect endothelial dysfunction.

METHODS We assessed endothelial and kidney function in women with a history of PE $(n=79)$ and uncomplicated pregnancies $(n=49)$ at least 4 years postpartum. Women with hypertension, diabetes or kidney disease prior to pregnancy were excluded. Brachial artery flow mediated dilatation (FMD) was measured and analysed by a custom designed edge-detection and wall-tracking software. We measured albumin and creatinine levels in a 24 hour urine sample and calculated glomerular filtration rate (GFR) by CKD-EPI.

RESULTS Women with a history of PE had lower FMD but comparable GFR and albumin creatinine ratio (ACR) compared to controls. Independent of obstetric history, in both controls and women with a history of PE respectively, GFR $(r=0.19, p=0.17$ and $r=0.12, p=0.29)$ and albumin creatinine ratio $(r=0.07, p=0.62$ and $r=0.06 p=$ $0.57)$ did not correlate with FMD.

CONCLUSION At least 4 years after pregnancy, women with a history of PE demonstrated decreased FMD when compared to healthy parous controls. In this study, decreased flow mediated dilation however did not coincide with decreased kidney function. 


\section{INTRODUCTION}

Preeclampsia (PE) is a pregnancy induced endothelial disease that is characterized by hypertension and albuminuria. Despite the low incidence of 2 - $8 \%$ of pregnancies complicated by PE, it has great impact on maternal and foetal morbidity and mortality ${ }^{1}$. Worldwide, PE accounts for $14 \%$ of maternal deaths, resultantly PE is the second most common cause of maternal death after postpartum haemorrhage ${ }^{2,3}$. In women with a history of PE, endothelial function, as measured by flow mediated dilatation (FMD) is decreased. It improves in the first few weeks postpartum but fails to normalize in the first years after gestation when compared with FMD levels measured in healthy parous controls 4-6. This indicates only partial reversal of the endothelial dysfunction after a pregnancy complicated by PE. Women with an early onset PE or recurrent PE have more pronounced endothelial dysfunction after pregnancy suggesting that attenuated endothelial function parallels severity of disease in pregnancy ${ }^{6}$. Endothelial dysfunction during a pregnancy complicated by PE also affects kidney function. Kidney dysfunction, reflected by either albuminuria or decreased glomerular filtration rate (GFR), can persist over a period of time after delivery, but most women with a history of preeclampsia maintain good kidney function 7,8. Nonetheless, a subgroup of women have persistently decreased kidney function long after their complicated pregnancy 9,10. It may be that, in these women, endothelial dysfunction is reflected in both attenuated kidney function and endothelial dependent FMD ${ }^{11}$. In this study we tested the hypothesis that in women with a history of PE, kidney function correlates with endothelial dependent FMD. To this end, we measured endothelial function by brachial FMD and kidney function by evaluating 24 hours micro albuminuria and GFR, at least 4 years postpartum, in both women with a history of PE as in a control group of women who had normotensive pregnancies. 


\section{METHODS}

In this study, we used a database of women with a history of PE ( $n=99)$ who had been tested several years after their pregnancy complicated by PE. Healthy parous controls ( $\mathrm{n}$ = 49), women with normotensive pregnancies, were recruited through advertisement. All women included were of Northern European Ancestry and completed a follow-up program in 2011. Evaluations were performed in the non-pregnant state at least 4 years after the first (index) gestation postpartum. The study was approved by the Medical Ethics Committee of the Radboud University Medical Centre Nijmegen (CMO 2010/245). All women gave written informed consent. PE was defined according to the criteria of the Report of the National High Blood Pressure Education Program Working Group on High Blood Pressure in Pregnancy ${ }^{12}$. Women were instructed to fast overnight, abstain from alcohol and caffeine for 16 hours and not to perform any exercise in the 24 hours preceding the measurements. A 24 hour urine sample was collected. Upon arrival (body) characteristics were measured and a full medical history was taken. A venous blood sample was taken for measurement of kidney function. Serum creatinine and urine creatinine were measured by an enzymatic colorimetric method (Architect, Abbott Laboratories, Abbott Park, IL, USA) and urine albumin by immunonefelometry (Dade Behring BN II Nefelometer, Siemens, Mississauga, Canada). We estimated GFR by the CKD-EPI equation for female and Caucasian, in which both formulas are dependent on serum creatinine. When serum creatinine was below or equal to $0.7 \mathrm{mg} / \mathrm{d}$ l we used $144 \times$ (serum creatinine / 0.7) $)^{\wedge-0.329} \times$ $0.993^{\wedge a g e}$. When serum creatinine was above $0.7 \mathrm{mg} / \mathrm{dl}$ we used $144 \times$ (serum creatinine / $0.7^{\wedge-1.209} \times 0.993^{\wedge a g e}$. Albuminuria was quantified by the albumin creatinine ratio (ACR) in the 24 hour urine sample. Blood pressure was recorded for a period of 30 minutes at 3 minute intervals using a semiautomatic oscillometric device in half-sitting position. Median values of 9 subsequent recordings were used for analysis. Measurements of FMD were taken as previously described by Scholten et al. ${ }^{13}$. All FMD measurements were performed under standardized conditions in a temperature controlled $\left( \pm 20^{\circ} \mathrm{C}\right)$, quiet room. Before starting the protocol, subjects rested for at least 15 minutes, the arm was in an extended position at $\sim 80^{\circ}$ from the torso and patients were in supine position. An automated system ensured a rapid inflation and deflation of the cuff (D.E. Hokanson, Bellevue, WA), with cuff size recommended for the arm circumference and positioned around the forearm, a few centimetres distal to the olecranon.

A 10-MHz multifrequency linear array probe attached to a high resolution ultrasound machine (T3000, Terason, Burlington, MA) was used to image the brachial artery in the distal third of the upper arm, 2 - $5 \mathrm{~cm}$ above the antecubital fossa. The continuous Doppler velocity was simultaneously measured with the ultrasound at an angle of $<60^{\circ}$. 
First a 1 minute baseline recording of brachial artery diameter velocity was measured. Thereafter the forearm cuff was inflated (> $200 \mathrm{mmHg}$ ) for 5 minutes. The diameter and flow assessments were done 30 seconds before deflation until 3 minutes after deflation. FMD analysis was done by custom designed edge-detection and wall-tracking software, independent of investigator bias. Peak diameter was automatically detected according to an algorithm that is described in detail elsewhere ${ }^{14}$. Reproducibility of FMD using this semi-automated software possesses a coefficient of variation of $6.7-10.5 \%$.

\section{STATISTICAL ANALYSIS}

All analyses were performed using SPSS version 21.0, property of IBM. Data were expressed as group means and standard deviation or medians and interquartile ranges. A p-value less than 0.05 was considered to indicate a statistical significant difference. An unpaired t-test was used to analyze differences between groups with normally distributed data. For non-normal distributed data, we used the Mann-Whitney $U$ test. Dichotomic data was analysed with a chi square test. Bivariate correlations were analysed by Spearman's test. A logistic regression was performed to correct for months postpartum, age and the use of antihypertensive drugs. 


\section{RESULTS}

A total of 128 women, 79 with a history of PE and 49 with an uncomplicated pregnancy were selected after exclusion of women who had hypertension ( $n=18)$, diabetes mellitus $(n=5)$ prior to the pregnancy. None had a known history of kidney disease prior to pregnancy (Table 1).

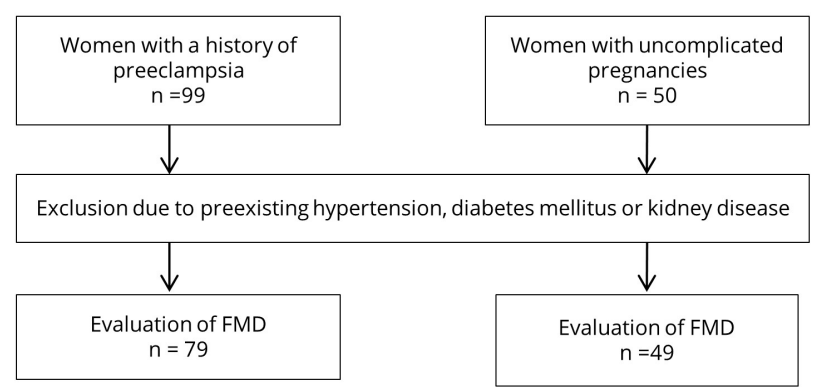

Table 1 Flowchart.

Three women with a history of PE and two healthy parous controls did not collect 24 hour urine. We did not estimate microalbuminuria in these subjects. Table 2 shows the characteristics of the study group. Women with a history of PE were on median 4.4 years postpartum, delivered at an earlier gestational age and gave birth to smaller infants at lower birth weight centile compared with healthy parous controls. Controls were on median 8.7 years postpartum and older compared to women with a history of PE. Women with a history of PE delivered in 53\% of cases before 34 weeks' gestation and 58\% had a child small for gestational age, defined as a growth below the 10th centile. FMD and GFR and micro-albuminuria were similar in women with PE with or without SGA and in women with PE and a delivery before or after 34 weeks pregnancy (see supplemental Tables 4 and 5). 


\begin{tabular}{llll} 
& $\begin{array}{l}\text { Uncomplicated } \\
\text { pregnancy } \\
\mathrm{n}=49\end{array}$ & $\begin{array}{l}\text { History of } \\
\text { preeclampsia } \\
\mathrm{n}=79\end{array}$ & $\mathrm{p}$-value \\
\hline Age (years) & $39 \pm 4$ & $35 \pm 4$ & $<0.001$ \\
\hline BMl (kg/m $\left.{ }^{2}\right)$ & $23 \pm 3$ & $25 \pm 6$ & 0.067 \\
\hline Smoking & $5(10 \%)$ & $8(9 \%)$ & 0.605 \\
\hline Primiparity & $5(10 \%)$ & $25(33 \%)$ & 0.009 \\
\hline Gestational age at delivery (weeks) & $39 \pm 2.3$ & $33 \pm 4.5$ & $<0.001$ \\
\hline Birth weight (gram) & $3358[3012-3745]$ & $1786[1036-2715]$ & $<0.001$ \\
\hline Birth centile & $44[20-65]$ & $24[5-34]$ & $<0.001$ \\
\hline Months postpartum & $96[70-119]$ & $59[47-66]$ & $<0.001$ \\
\hline Antihypertensive drugs & 0 & $7(9 \%)$ & 0.031 \\
\hline
\end{tabular}

Table 2 Baseline characteristics of women with a history of preeclampsia and uncomplicated pregnancy.

Women with a history of preeclampsia had lower FMD (6.6 \pm 3.0 vs. $9.0 \pm 3.6, p=0.001)$ and higher systolic blood pressure (114 $\pm 11 \mathrm{vs.} 110 \pm 10, p=0.005)$ but similar GFR (105 \pm 16 vs. $99 \pm 14, p=0.55)$ and albumin creatinine ratio (0.6 [0.3 - 1.3] vs. 0.5 [0.4 - 1.1], p $=0.92$ ) when compared with women with an uncomplicated pregnancy (Table 3).

\begin{tabular}{llll} 
& $\begin{array}{l}\text { Uncomplicated } \\
\text { pregnancy } \\
\mathrm{n}=49\end{array}$ & $\begin{array}{l}\text { History of } \\
\text { preeclampsia } \\
\mathrm{n}=79\end{array}$ & p-value \\
\hline GFR (m//min/1.73m2) & $98 \pm 14$ & $105 \pm 16$ & 0.55 \\
\hline Albumin creatinine ratio (mg/mmol) & $0.5[0.4-1.1]$ & $0.6[0.3-1.3]$ & 0.92 \\
\hline Flow mediated dilation (\%) & $9.0 \pm 3.6$ & $6.6 \pm 3.0$ & 0.001 \\
\hline Baseline diameter a. brachialis (mm) & $3.0 \pm 0.3$ & $3.0 \pm 0.4$ & 0.76 \\
\hline Systolic blood pressure (mmHg) & $110 \pm 10$ & $114 \pm 11$ & 0.005 \\
\hline Diastolic blood pressure $(\mathrm{mmHg})$ & $71 \pm 7$ & $72 \pm 8$ & 0.09 \\
\hline
\end{tabular}

Table 3 Kidney function, endothelial function and blood pressure in women with a history of preeclampsia and uncomplicated pregnancy. Data presented were adjusted for months postpartum, age and use of antihypertensive drugs.

Women with a history of PE also used more antihypertensive drugs ( $0 \%$ vs. $8.8 \%, p=$ $0.031)$. Baseline diameter values of the brachial artery between groups were comparable $(p=0.76)$. In both women with a history of PE and controls respectively, FMD did not correlate with either albumin creatinine ratio $(r=0.07, p=0.62$ and $r=0.07, p=0.51)$ or GFR ( $r=0.19, p=0.17$ and $r=0.15, p=0.19)$ (Figures 1 and 2 ). 


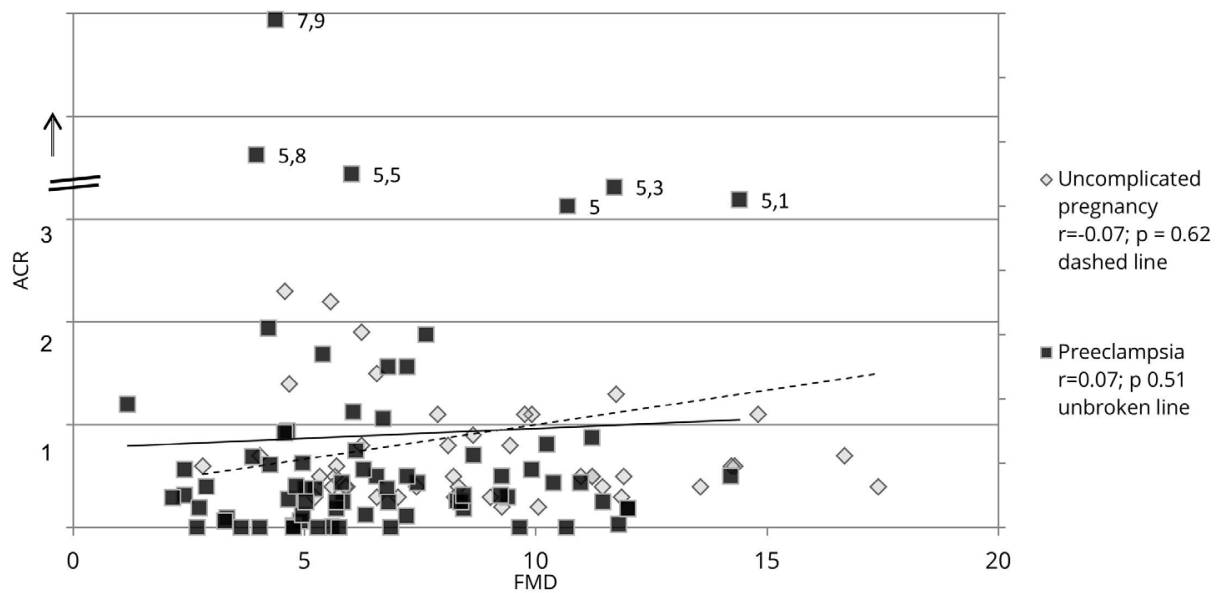

Figure 1 Correlation between albumin creatinine ratio and flow mediated dilatation (ACR/FMD) in women with a history of preeclampsia and uncomplicated pregnancy. The unbroken trend line represents women with preeclampsia and the dashed represents controls.

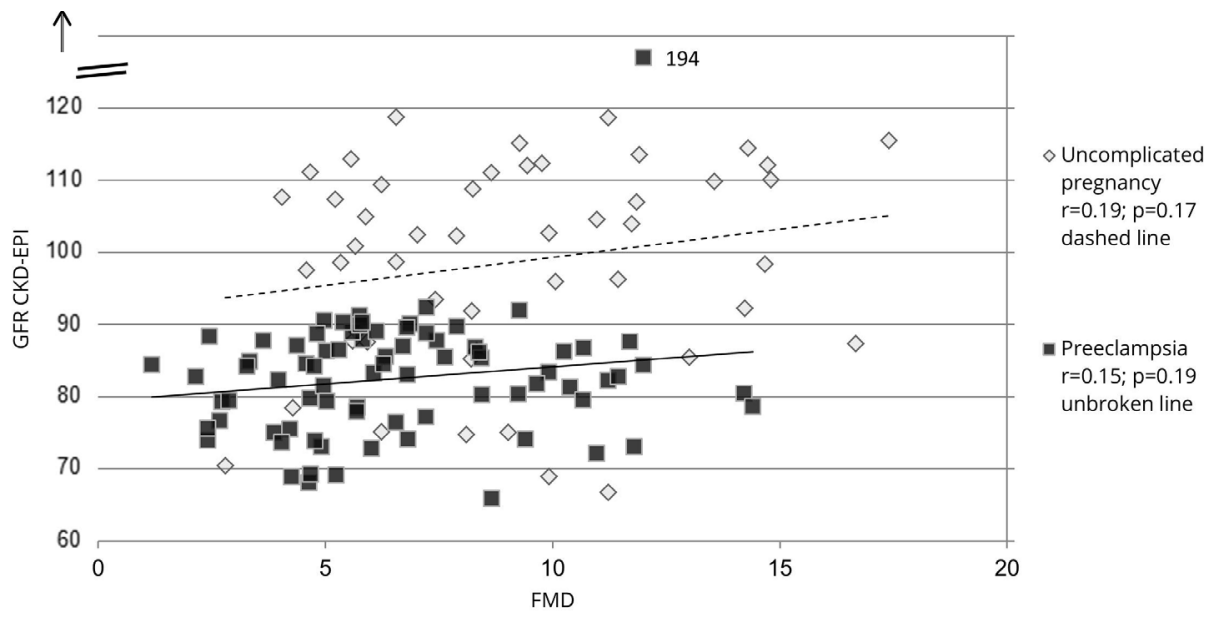

Figure 2 Correlation between glomerular filtration rate and flow mediated filtration (GFR/FMD) in women with a history of preeclampsia and uncomplicated pregnancy. The unbroken trend line represents women with preeclampsia and the dashed represents controls. 


\section{DISCUSSION}

Women with a history of PE have decreased FMD, suggesting endothelial dysfunction, but demonstrate similar GFR and urinary albumin loss when compared to healthy parous controls. In contrast to our expectations, we observed no correlation between FMD and kidney function. Interestingly a correlation has been found between a decreased FMD and proteinuria at 5 years postpartum in women with a history of PE ${ }^{15}$. We were, however, unable to find a correlation between GFR and FMD on the one hand and microalbuminuria and FMD on the other. This could be a result of a different postpartum interval, chosen exclusion criteria or differences in the severity of the gestational disease. A sub-analysis of women with an increased ACR and FMD was not possible due to the small number of women.

Damaged endothelium frequently results in altered permeability of vessels, in the glomerulus; this is clinically apparent as albuminuria ${ }^{16}$. The absence of micro albuminuria, a variable that is considered to reflect endothelial disease, particularly if GFR is normal, might suggest that there is endothelial recovery in the kidneys, but that women with a history of PE could have constitutional reduced FMD or a higher susceptibility for endothelial dysfunction as compared to healthy parous controls. Chronic kidney disease in itself is associated with a decreased endothelial function as measured by FMD ${ }^{17-19}$. Even though decreased FMD and microalbuminuria both reflect endothelial dysfunction, they may reflect different aspects of endothelial function. Alternatively endothelial function as assessed by micro-albuminuria may follow a different recovery rate than endothelial function expressed with FMD.

The vaso homeostasis of our endothelium is primarily effectuated by nitric oxide which is released in response to increased shear stress caused by changes in blood flow. When the endothelium loses its ability to maintain the delicate balance of circulatory, haemostatic and immunological homeostasis, it becomes vulnerable for the invasion of lipids and leukocytes at locations where lesions occur. A dysfunctioning endothelium, and a decreased availability of nitric oxide, is considered the first step in the development of atherosclerosis and subsequently cardiovascular disease.

Although time consuming, FMD is a useful non-invasive reproducible ultrasonographic technique that has been used in many clinical studies and is an indicator of vascular health ${ }^{20,21}$.

During preeclampsia, FMD is decreased, indicating endothelial dysfunction ${ }^{22-25}$. During pregnancy, this dysfunction is paralleled by increased permeability leading to extravasation 
of fluid and oedema formation on the one hand, and the loss of albumin due to glomerular endotheliosis on the other. The endothelium not only seems to be attenuated during the pregnancy affected by $P E$, but this seems to persist until several years postpartum 6,26-29. The relative difference of FMD between women with a history of PE and controls is generally between 20 - 58\%, which is similar in our study ${ }^{26-28}$.

Endothelial dysfunction is also expressed by a decreased kidney function during a pregnancy complicated by PE and thereafter. Women with PE exhibit increased albuminuria during pregnancy and have a 4 to 5 times increased risk of end stage renal disease in later life 30. Some women maintain a decreased kidney function after PE. This could be either due to an undiagnosed pre-existing kidney disease, higher susceptibility or concomitant renal risk factors in these women to develop chronic kidney disease or due to irreversible kidney damage as a consequence of PE. Most studies on kidney function after PE have insufficient data on pre-pregnancy GFR or albuminuria values to determine a possible subclinical undetected pre-existing kidney dysfunction ${ }^{10}$. Despite the fact that we excluded women with known pre-existing renal disease, we cannot rule out subtle abnormalities in renal function. Nonetheless, the observed kidney function does not support the thought that the population studied in this study suffered from kidney dysfunction. Even though some studies show decreased kidney function after pregnancy complicated by PE ${ }^{31}$, others do not 32,33 . Nonetheless, as a group, women with a history of PE have an increased risk of end stage renal disease, even when corrected for common risk factors and confounders such as pre-existing kidney disease, rheumatic disease, hypertension, or diabetes mellitus before pregnancy ${ }^{34}$. Similarly, FMD appears to be diminished in several, but not all studies in women with a history of PE 6,32,35.

It is important to note that all these studies are heterogeneous in nature due to multiple factors namely, measurements at a certain point in time after delivery, differences in severity of PE and in treatment during pregnancy, but also because of different health policies instituted after pregnancy. It is therefore difficult to draw conclusions based on such heterogeneous studies on the pattern of recovery.

Endothelial dysfunction, either expressed by albuminuria or decrease in FMD, has been investigated in several prospective studies in relation to cardiovascular disease ${ }^{36-38}$. Meta-analysis detailed that an absolute increase in FMD by $1 \%$ decreased the risk of a cardiovascular event in the upcoming 8 years by $13 \%{ }^{39}$. Moreover, a two-fold increase in albuminuria, another predictor of cardiovascular mortality, is associated with 29\% more risk for cardiovascular mortality ${ }^{40}$. Therefore, it seems prudent to evaluate FMD and micro-albuminuria in the follow-up of women with a history of PE as both are considered markers that indicated the risk of future cardiovascular disease. 
There are some shortcomings in this study that need to be addressed. First, on the one hand, our population contains predominantly women with either early onset disease or concomitant small for gestational age infancy, both indicators considered to represent more severe disease. This may limit generalizability of our results to all women with a history of PE. On the other hand, the results might be an underestimation of the prevalence of chronic kidney disease as we excluded women with pre-existing diabetes mellitus, hypertension and kidney disease. Second, the cross-sectional nature of this study may have affected observations. It may be that pace in recovery from pregnancy may be different in the kidney as compared to the endothelium in general, reflected by FMD, which could only be detected by repeated measures over time.

\section{CONCLUSION}

Several years after gestation, women with a history of PE have decreased endothelial function as measured by FMD. The decreased FMD does relate neither to decreased glomerular filtration rate nor to albuminuria. 


\section{APPENDIX}

\begin{tabular}{|c|c|c|c|}
\hline & $\begin{array}{l}\mathrm{PE}<34 \text { weeks } \\
\mathrm{n}=42\end{array}$ & $\begin{array}{l}\text { PE }>34 \text { weeks } \\
n=37\end{array}$ & p-value \\
\hline $\mathrm{GFR}\left(\mathrm{ml} / \mathrm{min} / 1.73 \mathrm{~m}^{2}\right)$ & $104 \pm 14$ & $107 \pm 19$ & 0.290 \\
\hline Albumin creatinine ratio ( $\mathrm{g} / \mathrm{mmol})$ & $1.5 \pm 2.6$ & $1.3 \pm 2.4$ & 0.655 \\
\hline Flow mediated dilation (\%) & $7.1 \pm 2.7$ & $6,2 \pm 3,2$ & 0.316 \\
\hline Baseline diameter a. brachialis ( $\mathrm{mm}$ ) & $3.1 \pm 0.4$ & $3,0 \pm 0,5$ & 0.950 \\
\hline Systolic blood pressure (mmHg) & $116 \pm 12$ & $113 \pm 10$ & 0.116 \\
\hline Diastolic blood pressure (mmHg) & $74 \pm 8$ & $70 \pm 8$ & $0.029 *$ \\
\hline
\end{tabular}

Supplemental Table 4 Kidney function, endothelial function and blood pressure in women with a history of preeclampsia (PE) subdivided by small for gestational age (SGA). Data presented were adjusted for months postpartum, age and use of antihypertensive drugs.

\begin{tabular}{llll} 
& $\begin{array}{l}\text { PE without SGA } \\
\mathrm{n}=46\end{array}$ & $\begin{array}{l}\text { PE with SGA } \\
\mathrm{n}=33\end{array}$ & p-value \\
\hline GFR $\left(\mathrm{ml} / \mathrm{min} / 1.73 \mathrm{~m}^{2}\right)$ & $106 \pm 18$ & $103 \pm 14$ & 0.231 \\
\hline Albumin creatinine ratio $(\mathrm{g} / \mathrm{mmol})$ & $1.5 \pm 2.9$ & $1.3 \pm 1.7$ & 0.457 \\
\hline Flow mediated dilation $(\%)$ & $6.2 \pm 2.8$ & $7.2 \pm 3.1$ & 0.279 \\
\hline Baseline diameter a. brachialis $(\mathrm{mm})$ & $3.0 \pm 0.4$ & $3.0 \pm 0.4$ & 0.608 \\
\hline Systolic blood pressure $(\mathrm{mmHg})$ & $113 \pm 12$ & $117 \pm 10$ & 0.138 \\
\hline Diastolic blood pressure $(\mathrm{mmHg})$ & $71 \pm 8$ & $74 \pm 7$ & 0.34 \\
\hline
\end{tabular}

Supplemental Table 5 Kidney function, endothelial function and blood pressure in women with a history of preeclampsia (PE) subdivided by early and late onset. Data presented were adjusted for months postpartum, age and use of antihypertensive drug. 


\section{REFERENCES}

1. Ghulmiyyah L, Sibai B. Maternal mortality from preeclampsia/eclampsia. Semin. Perinatol. Feb 2012;36(1):56-59.

2. WHO. The World health report: make every mother and child count. 2005.

3. Say L, Chou D, Gemmill A, et al. Global causes of maternal death: a WHO systematic analysis. Lancet Global Health. Jun 2014;2(6):E323-E333.

4. Higashi Y. Assessment of endothelial function. History, methodological aspects, and clinical perspectives. Int. HeartJ. 2015;56(2):125-134.

5. Kuscu NK, Kurhan Z, Yildirim Y, Tavli T, Koyuncu F. Detection of endothelial dysfunction in preeclamptic patients by using color Doppler sonography. Arch. Gynecol. Obstet. Jun 2003;268(2):113-116.

6. Chambers JC, Fusi L, Malik IS, Haskard DO, De Swiet M, Kooner JS. Association of maternal endothelial dysfunction with preeclampsia. JAMA. Mar 28 2001;285(12):1607-1612.

7. Lafayette RA, Druzin M, Dibley R, et al. Nature of glomerular dysfunction in pre-eclampsia. Kidney Int. 1998;54:1240-1249.

8. Hussein W, Lafayette RA. Renal function in normal and disordered pregnancy. Curr. Opin. Nephrol. Hypertens. Jan 2014;23(1):46-53.

9. Spaan JJ, Ekhart T, Spaanderman ME, Peeters LL. Remote hemodynamics and renal function in formerly preeclamptic women. Obstet. Gynecol. Apr 2009;113(4):853-859.

10. McDonald S, Han Z, Walsh M, Gerstein H, Devereaux P. Kidney Disease After Preeclampsia: A Systematic Review and Meta-analysis. Am. J. Kidney Dis. 2010;55(6):1026-1039.

11. Deckert T, Feldt-Rasmussen B, Borch-Johnsen $K$, Jensen $T$, Kofoed-Enevoldsen A. Albuminuria reflects widespread vascular damage. The Steno hypothesis. Diabetologia. 1989(32):219-226.

12. Report of the National High Blood Pressure Education Program Working Group on High Blood Pressure in Pregnancy. Am. J. Obstet. Gynecol. 2000;183(1):s1-s22.

13. Scholten RR, Spaanderman MEA, Green DJ, Hopman MT, Thijssen DH. Retrograde shear rate in formerly preeclamptic and healthy women before and after exercise training: relationship with endothelial function. Am. J. Physiol. Heart Circ. Physiol. Aug 01 2014;307(3):H418-425.

14. Black MA, Cable NT, Thijssen DH, Green DJ. Importance of measuring the time course of flow-mediated dilatation in humans. Hypertension. Feb 2008;51(2):203-210.

15. Aykas F, Solak Y, Erden A, et al. Persistence of cardiovascular risk factors in women with previous preeclampsia: a long-term follow-up study. J. Investig. Med. Apr 2015;63(4):641-645.

16. Salmon AH, Satchell SC. Endothelial glycocalyx dysfunction in disease: albuminuria and increased microvascular permeability. J. Pathol. Mar 2012;226(4):562-574.

17. Malyszko J. Mechanism of endothelial dysfunction in chronic kidney disease. Clin. Chim. Acta. Oct 09 2010;411(19-20):1412-1420.

18. Verbeke FH, Agharazii M, Boutouyrie P, Pannier B, Guerin AP, London GM. Local shear stress and brachial artery functions in end-stage renal disease. J. Am. Soc. Nephrol. Feb 2007;18(2):621-628.

19. Verbeke FH, Pannier B, Guerin AP, Boutouyrie P, Laurent S, London GM. Flow-mediated vasodilation in endstage renal disease. Clin. J. Am. Soc. Nephrol. Aug 2011;6(8):2009-2015.

20. Thijssen DH, Black MA, Pyke KE, et al. Assessment of flow-mediated dilation in humans: a methodological and physiological guideline. Am. J. Physiol. Heart Circ. Physiol. Jan 2011;300(1):H2-12.

21. Atkinson G, Batterham AM. The clinical relevance of the percentage flow-mediated dilation index. Curr. Hypertens. Rep. Feb 2015;17(2):4.

22. Guimaraes MF, Brandao AH, Rezende CA, et al. Assessment of endothelial function in pregnant women with preeclampsia and gestational diabetes mellitus by flow-mediated dilation of brachial artery. Arch. Gynecol. Obstet. Sep 2014;290(3):441-447.

23. Dorup I, Skajaa K, Sorensen KE. Normal pregnancy is associated with enhanced endothelium-dependent flow-mediated vasodilation. Am. J. Physiol. 2. 1999;276: H821-H825.

24. Matias Costa Vieira, Edson Vieira da Cunha Filho, Letícia Germany Paula, Marta Ribeiro Hentschke, Carlos Eduardo Poli-de-Figueiredo, Costa BEPd. Flow-mediated dilatation of brachial artery as marker of preeclampsia morbidity. Int. J. Cardiol. 2013;168:4424-4425.

25. Weissgerber TL. Flow-mediated dilation: can new approaches provide greater mechanistic insight into vascular dysfunction in preeclampsia and other diseases? Curr. Hypertens. Rep. Nov 2014;16(11):487. 
26. Hamad R, Eriksson MJ, A S, Hamsten A, Bremmea K. Decreased flow-mediated dilation is present 1 year after a pre-eclamptic pregnancy. J. Hypertens. 2007;25(2301-2307).

27. Paez O, Alfie J, Gorosito M, et al. Parallel decrease in arterial distensibility and in endothelium-dependent dilatation in young women with a history of pre-eclampsia. Clin. Exp. Hypertens. Oct 2009;31(7):544-552.

28. Goynumer G, Yucel N, Adali E, Tan T, Baskent E, Karadag C. Vascular risk in women with a history of severe preeclampsia. J. Clin. Ultrasound. Mar-Apr 2013;41(3):145-150.

29. Murphy MS, Vignarajah M, Smith GN. Increased microvascular vasodilation and cardiovascular risk following a pre-eclamptic pregnancy. Physiol Rep. Nov 01 2014;2(11).

30. Vikse BE. Pre-eclampsia and the risk of kidney disease. The Lancet. 2013;382(9887):104-106.

31. Bar J, Boris Kaplan, Clara Wittenberg, et al. Microalbuminuria after pregnancy complicated by pre-eclampsia. Nephrol. Dial. Transplant. 1999(14):1129-1132.

32. Sandvik MK, Hallan S, Svarstad E, Vikse BE. Preeclampsia and prevalence of microalbuminuria 10 years later. Clin. J. Am. Soc. Nephrol. Jul 2013;8(7):1126-1134.

33. Lampinen KH, Ronnback M, Groop PH, Kaaja RJ. Renal and vascular function in women with previous preeclampsia: a comparison of low- and high-degree proteinuria. Kidney Int. Nov 2006;70(10):1818-1822.

34. Vikse BE, Irgens LM, Leivestad T, Skjaerven R, Iversen BM. Preeclampsia and the risk of end-stage renal disease. N. Engl. J. Med. Aug 21 2008;359(8):800-809.

35. Sandvik MK, Leirgul E, Nygard O, et al. Preeclampsia in healthy women and endothelial dysfunction 10 years later. Am. J. Obstet. Gynecol. Dec 2013;209(6):569 e561-569 e510.

36. Ras RT, Streppel MT, Draijer R, Zock PL. Flow-mediated dilation and cardiovascular risk prediction: a systematic review with meta-analysis. Int. J. Cardiol. Sep 20 2013;168(1):344-351.

37. Frick M, Suessenbacher A, Alber HF, et al. Prognostic value of brachial artery endothelial function and wall thickness. J. Am. Coll. Cardiol. Sep 20 2005;46(6):1006-1010.

38. Maruhashi T, Soga J, Fujimura N, et al. Relationship between flow-mediated vasodilation and cardiovascular risk factors in a large community-based study. Heart. Dec 2013;99(24):1837-1842.

39. Inaba Y, Chen JA, Bergmann SR. Prediction of future cardiovascular outcomes by flow-mediated vasodilatation of brachial artery: a meta-analysis. Int. J. Cardiovasc. Imaging. Aug 2010;26(6):631-640.

40. Hillege HL. Urinary Albumin Excretion Predicts Cardiovascular and Noncardiovascular Mortality in General Population. Circulation. 2002;106(14):1777-1782. 


\title{
CHAPTER 7
}

\section{Vascular aging in young and middle-aged women after a hypertensive complicated and uncomplicated pregnancy}

\author{
Veronica A. Lopes van Balen \\ Chahinda Ghossein-Doha \\ Julia J. Spaan \\ Eva G. Mulder \\ Sander M.J. van Kuijk \\ Erjona Morina-Shijaku \\ Hester M. den Ruijter \\ Gerard Pasterkamp \\ Anton Jan van Zonneveld \\ Abraham A. Kroon \\ Vanessa van Empel \\ Hans-Peter Brunner LaRocca \\ Arnold P. Hoeks \\ Koen D. Reesink \\ Marc E.A. Spaanderman
}




\section{ABSTRACT}

INTRODUCTION Arterial ageing, characterized by decreased endothelial function, raises the risk of cardiovascular disease (CVD). During hypertensive complicated pregnancy, women have decreased endothelial function and an increased future risk of CVD compared to uncomplicated pregnancies. We tested the hypothesis that the age-related decline in endothelial function is attenuated in women with a history of hypertensive complicated pregnancy compared to uncomplicated pregnancies.

METHODS We performed a case-control study in which we included women with hypertensive complicated and uncomplicated pregnancies 0.5 to 30 years thereafter. Arterial aging was assessed by brachial artery flow-mediated dilation (FMD) and sublingual nitroglycerine. To analyse the cross-sectional age-related decline in endothelial function we performed a multivariable adjusted logistic regression.

RESULTS We analysed 391 cases and 237 controls (23 to 61 years of age). FMD did not differ between groups and remained unaltered when age increased. In both groups nitroglycerine dilation decreased $(-0.075 \% / y e a r, p=0.008)$ and brachial artery diameter increased comparably $(0.014 \mathrm{~mm} / \mathrm{year}, \mathrm{p}<0.001)$ over time. When adjusted for confounders known to affect endothelial function, the age-associated enlargement of brachial artery diameter over time remained $(0.012 \mathrm{~mm} / \mathrm{year}, \mathrm{p}<0.001)$ unlike the association with nitroglycerine dilation.

CONCLUSION In young to middle-aged women, irrespective of obstetric history, there is an age-related decline in nitroglycerine induced dilation and increase in brachial artery diameter. However, FMD did not change within age groups regardless of obstetric history. The increased CVD risk in women with hypertensive complicated pregnancy in their history does not to go hand in hand with a decline in FMD or nitroglycerine dilation. 


\section{INTRODUCTION}

Arterial aging is a physiological process that develops gradually over time and increases the risk of cardiovascular disease (CVD). With advancing age, several arterial changes contribute to this increased risk ${ }^{1,2}$. One of the most clinically important changes in the development of vascular dysfunction is a decline in endothelial function observed as an impaired dilatory capacity of the vessel. The dilatory capacity of arteries is mediated by endogenous nitric oxide (NO) release by the endothelium and consequent relaxation of vascular smooth muscle ${ }^{3-5}$. Underlying this physiological response, whether or not related to (transient) distal hyperaemia, is the process of autoregulation keeping local wall shear stress constant 6 .

Flow-mediated dilatation (FMD) is commonly used to assess endothelial function. FMD of the brachial artery can be quantified non-invasively by high-resolution ultrasound. Diminished FMD is associated with CVD and is therefore used as proxy for cardiovascular health 7-10. Endothelium-independent dilation can be assessed by bypassing the endogenous endothelial NO release through administration of exogenous (sublingual) nitroglycerine (NG). This allows measurement of the maximum capacity to dilate.

Besides age, a decline in endothelial function may be accelerated by both conventional and sex-specific cardiovascular risk factors ${ }^{11-14}$. Preeclampsia (PE), a hypertensive complication of pregnancy, is mostly marked by blunted endothelial function, not only during but also in the first years after pregnancy ${ }^{15,16}$. PE is shown to increase the risk of CVD 2 - 7 fold and can therefore be considered an early sex-specific cardiovascular risk factor ${ }^{17-19}$. In the present study, we hypothesize that the age-related decline in endothelial function is more pronounced in women with a hypertensive complicated pregnancy in their history compared to women who experienced uncomplicated pregnancies. 


\section{METHODS}

This study is part of a large cohort study to investigate subclinical heart failure in women (Queen of Hearts study; ClinicalTrials.gov Identifier: NCT02347540) and was approved by the Medical Ethics Committee of the Maastricht University Medical Centre (MEC azM/UM 14-2-20136 NL 47252.068.14). All participating women gave written informed consent. The procedures were in conformity with institutional guidelines and adhered to the principles of the Declaration of Helsinki and Title 45, U.S. Code of Federal Regulation, Part 46, Protection of Human Subjects, Revised October 2013, Fortaleza, Brazil.

Between December 2014 and February 2017 we included women with a history of a vascular complicated pregnancy, namely PE, and women with a history of normotensive pregnancies. We recruited our study population by actively inviting by e-mail or postal mail, women who had previously undergone a preconceptional consultation in our tertiary hospital. The control group was included by advertising on social media, in local newspapers and on national television. Our inclusion criteria for the study population were a history of PE (not confined to any specific pregnancy), more than 6 months up to 30 years ago and being older than 18 years of age. The control group had similar inclusion criteria, but required having had a normotensive pregnancy in absence of any kind of placental associated disease (hypertensive complicated pregnancy, PE, HELLP syndrome, placental abruption, and small for gestational age infancy or foetal demise). Exclusion criteria for both groups were hypertension, auto-immune disease, or kidney disease prior to the first pregnancy. Participants were allowed to be diagnosed with any of these conditions after their first pregnancy. PE was defined according to the criteria of the Report of the National High Blood Pressure Education Program Working Group on High Blood Pressure in Pregnancy ${ }^{20}$. Hypertension was defined a systolic blood pressure $\geq 140$ and/or diastolic blood pressure $\geq 90 \mathrm{mmHg}$. A positive family history of CVD was defined by a parent or sibling below the age of 65 years with CVD ${ }^{21}$.

\section{MEASUREMENTS}

Cardiovascular analysis was performed in combination with endothelium-dependent and endothelium-independent evaluation of brachial artery dilation, with FMD and a sublingual dose of NG, respectively. Women were instructed to fast overnight, for at least 10 hours. Upon arrival (body) characteristics were measured, blood samples were drawn and a complete (obstetrical) medical history was taken. Neonatal birth weight centile was determined using Dutch reference curves for birth weight ${ }^{22}$. Height and weight were measured to calculate body mass index (BMI). Glucose was determined by standard hospital laboratory procedures using a Cobas 8000 device (Roche Diagnostics, Indianapolis, IN). 
Blood pressure was recorded for a period of 30 minutes at 3-minute intervals using an oscillometric device (Carescape V100, GE Healthcare, Wisconsin, USA) in half-sitting position. Median values of 9 subsequent recordings were used for analysis.

FMD and NG-dilation measurements were performed sequentially under standardized conditions in a temperature controlled $\left( \pm 22{ }^{\circ} \mathrm{C}\right.$ ) room. Before the measurement, participants rested in supine position on a comfortable bed for at least 15 minutes. The arm was in an extended position at $\sim 80^{\circ}$ from the torso. A rapid inflation and deflation cuff (Hokanson, Bellevue, VA 98005) was positioned around the forearm distal to the olecranon. A multifrequency linear array probe attached to a high resolution ultrasound machine (Voluson p6, GE Healthcare) with an operating frequency of $5 \mathrm{MHz}$ was used to image the brachial artery in the distal third of the upper arm, $2-5 \mathrm{~cm}$ above the antecubital fossa. The probe was fixed during all measurement by a custom-made fixation device made by hospital technicians. For the endothelium-dependent evaluation we acquired a 3-minute baseline recording of brachial artery diameter. Thereafter, the forearm cuff was inflated (200 $\mathrm{mmHg}$ ) for 5 minutes followed by rapid deflation. The diameter and Doppler spectrum assessments were done continuously from 3 minutes before inflation to 5 minutes after deflation, but interrupted from 30 seconds after inflation to 30 seconds before deflation.

Image analysis of the brachial artery diameter was done off-line with a custom designed edge-detection and wall-tracking software ${ }^{6}$, a proprietary algorithm by A. Hoeks (Matlab R2013b, The Mathworks Inc. Natick, MA), independent of investigator bias. Peak diameter was automatically detected according to an algorithm that is described in detail elsewhere ${ }^{6}$. The percentage increase in diameter, i.e. FMD, was based on the peak change in diameter with respect to leading baseline, with leading referring to the period before cuff-release.

Two experienced ultra-sonographers performed these measurements. In our hands, the inter-observer agreement, quantified as the intraclass correlation coefficient, was 0.82 , and the intra-observer agreement was 0.83 . This was calculated based on repeated measurements in 15 healthy volunteers by the two sonographers.

The NG evoked dilation also started with a 3 min baseline recording after which a dose of nitroglycerine (0.4 mg/dose) was administered sublingually. The recording ended $10 \mathrm{~min}$ after sublingual administration of NG and was analysed by the same custom designed edge-detection and wall-tracking software. 
FMD was also expressed with an allometric scaling as proposed by Atkinson et el. to avoid the baseline dependency observed in previous studies ${ }^{23}$. The regression slope between the logarithmically transformed values of both baseline diameter and peak diameter yielded a 0.99 scaling exponent; which was used to calculate the allometric scaled FMD.

\section{STATISTICAL ANALYSIS}

All analyses were performed using IBM SPSS version 21.0. Data were expressed as group means and standard deviation or, in case of dichotomous measurements, as numbers and percentages. To analyse differences in means of baseline characteristics between women with a history of PE and controls, we used the independent t-test and a chi square test for dichotomous variables. We compared the baseline characteristics of those women who did not undergo FMD measurements due to logistic reasons or artefacts with women that did undergo FMD.

To analyse the primary outcome we performed a multivariable linear regression analysis to evaluate the effect of age, group, and the interaction between age and group on endothelial function. In case the interaction term was not statistically significant, this term was omitted from the model. Subsequently, if group (control or case) was not significant it was also removed from the model. We computed both the unadjusted coefficients, and the coefficients after adjusting for known factors that affect endothelial function, namely smoking, multivitamin use, use of anti-hypertensive drugs, mean arterial pressure, fasting glucose levels and menopausal state ${ }^{4}$. These factors were chosen because of their known negative effect on FMD measurements. To evaluate the effect of postpartum interval on the primary outcomes a post hoc analysis was done equal to the primary analysis whereby age was replaced by postpartum interval.

A stepwise backward multivariable linear regression was used to estimate the effect of above mentioned, literature based, factors that might influence endothelial function in our sample. For the allometric FMD, a method to compensate for potential differences in vessel diameter, we calculated the regression slope between logarithmically transformed values of both baseline diameter and peak diameter and derived the correct scaling exponent for our dataset ${ }^{23}$. A p $<0.05$ is considered significant and signalled by an asterisk. 


\section{RESULTS}

In the above mentioned time period we included 673 women for the study. Due to logistical circumstances, $6.4 \%$ of vessel function tests could not be performed; these women were not included in the analysis. This resulted in 628 women included, 391 cases and 237 controls. There was no difference in patient characteristics between women that did or did not undergo endothelial function measurements. A small percentage (6.3\%) of FMD/ NG recordings were excluded, because of movement artefacts rendering wall detection unreliable.

Table 1 shows participants characteristics for both the study group and the control group. Women with a hypertensive complicated pregnancy in their history were on average 6 years younger and had their measurements performed at a shorter postpartum interval compared to controls. The study group had on average given birth to smaller babies ( $\Delta$ 22 centile, $p<0.001$ ) born at an earlier gestational age compared to controls ( $\Delta 4$ weeks and 6 days, $\mathrm{p}<0.001$ ). At the time of measurement, both groups had a similar BMI and a comparable fasting glucose, but mean arterial blood pressure was higher in women with a history of PE compared to controls $(p<0.001)$. In addition, women with a history of PE had more hypertension (17.4\% vs. 8.9\%; $p=0.001)$, used more antihypertensive drugs compared to controls (12.3\% vs. $7.2 \%$; $p=0.019)$, were more likely to have given birth only once (42.2\% vs. 18.9\%; $p$ < 0.001), and used less often supplemental multivitamins $(p=0.028)$. Nicotine use was comparable between both groups ( $p=0.094)$. A positive family history of CVD did not differ substantially between cases and controls ( $p=0.353$ ).

The development of new onset auto-immune disease and diabetes mellitus was not different between both groups (19 (5.1\%) cases and 6 (2.8\%) controls, $p=0.148$ and 6 (2\%) cases and 1 (0.5\%) control, $p=0.198$, respectively).

Figures 1 and 2 show data on baseline brachial artery diameter (mm), FMD (\%), allometric flow mediated dilation (FMD allometric, \%) and endothelium independent dilation induced by exogenous nitroglycerine (NG, \%) for cases and controls subdivided by age interval and Table 2 shows results of the statistical analysis to detect differences.

Table 2a presents the results of the multivariable linear regression to analyse the effect of time, group, and the interaction between time and group. We did not observe an agerelated change in FMD in both groups (regression coefficient $(\beta)-0.006(-0.039-0.028), p$ $=0.747)$. The allometric scaled FMD showed similar results as compared to the traditional FMD. NG evoked dilation decreased significantly in both groups with age ( $\beta-0.075$ (-0.131 - -0.020), $p=0.008$ ) and baseline brachial artery diameter increased significantly with age 
( $\beta 0.014(0.009-0.019), p<0.001)$. There was no significant effect of group on any variable or of the interaction between age and group.

\begin{tabular}{llll} 
& $\begin{array}{l}\text { Cases } \\
\mathrm{n}=391\end{array}$ & $\begin{array}{l}\text { Controls } \\
\mathrm{n}=237\end{array}$ & p- value \\
\hline Age (years) & $39 \pm 8$ & $45 \pm 8$ & $<0.001^{*}$ \\
\hline Months postpartum & $111 \pm 90$ & $197 \pm 99$ & $<0.001^{*}$ \\
\hline BMI (kg/m²) & $26 \pm 5$ & $25 \pm 4$ & 0.119 \\
\hline Gestational age at delivery (weeks) & $35^{+0} \pm 4^{+4}$ & $39+6 \pm 3^{+0}$ & $<0.001^{*}$ \\
\hline Birth weight centile & $32 \pm 26$ & $54 \pm 28$ & $<0.001^{*}$ \\
\hline Mean arterial pressure (mmHg) & $90 \pm 11$ & $86 \pm 9$ & $<0.001^{*}$ \\
\hline Glucose (mmol/L) & $5.0 \pm 0.8$ & $5.0 \pm 0.4$ & 0.173 \\
\hline Smoking & $25(6.4 \%)$ & $24(10 \%)$ & 0.091 \\
\hline Primiparity & $165(42.2 \%)$ & $45(18.9 \%)$ & $<0.001^{*}$ \\
\hline Antihypertensive drugs & $52(12.3 \%)$ & $17(7.2 \%)$ & $0.017^{*}$ \\
\hline Hypertension & $68(17.4 \%)$ & $21(8.9 \%)$ & $0.003^{*}$ \\
\hline Multivitamin use & $109(27.9 \%)$ & $86(36.2 \%)$ & $0.027^{*}$ \\
\hline Postmenopausal & $44(11.3 \%)$ & $52(21.9 \%)$ & $<0.001^{*}$ \\
\hline Positive family history for CVD & $241(61 \%)$ & $137(58 \%)$ & 0.610 \\
\hline
\end{tabular}

Table 1 Patient characteristic of women with a history of PE (Cases), and uncomplicated pregnancies (Controls) in means and standard deviation or numbers and percentages. CVD = Cardiovascular disease. An independent T-test analysed the differences between groups and a chi square test analysed dichotomous variables.

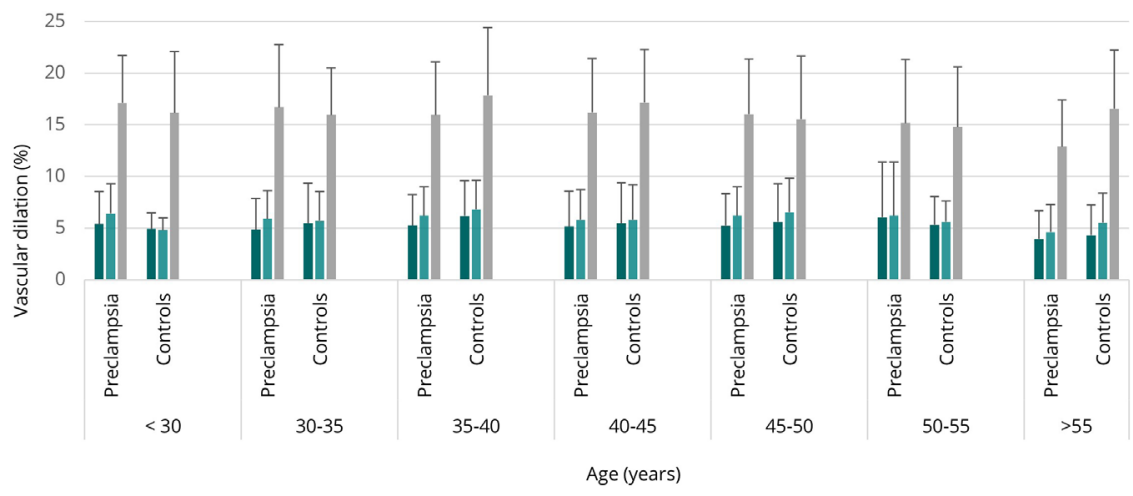

- Flow mediated dilation (FMD) $\quad$ FMD allometric Nitroglycerine induced dilation (NG)

Figure 1 In women with a history of PE (Cases) and uncomplicated pregnancies (Controls) brachial artery endothelium-independent dilation as measured by NG induced dilation declines with increasing age while flow mediated dilation (FMD, \%) and allometric flow mediated dilation (FMD allometric, \%) do not change (for statistical analysis see Table $2 \mathrm{a}$ and b). Data are expressed in mean and standard deviation. 


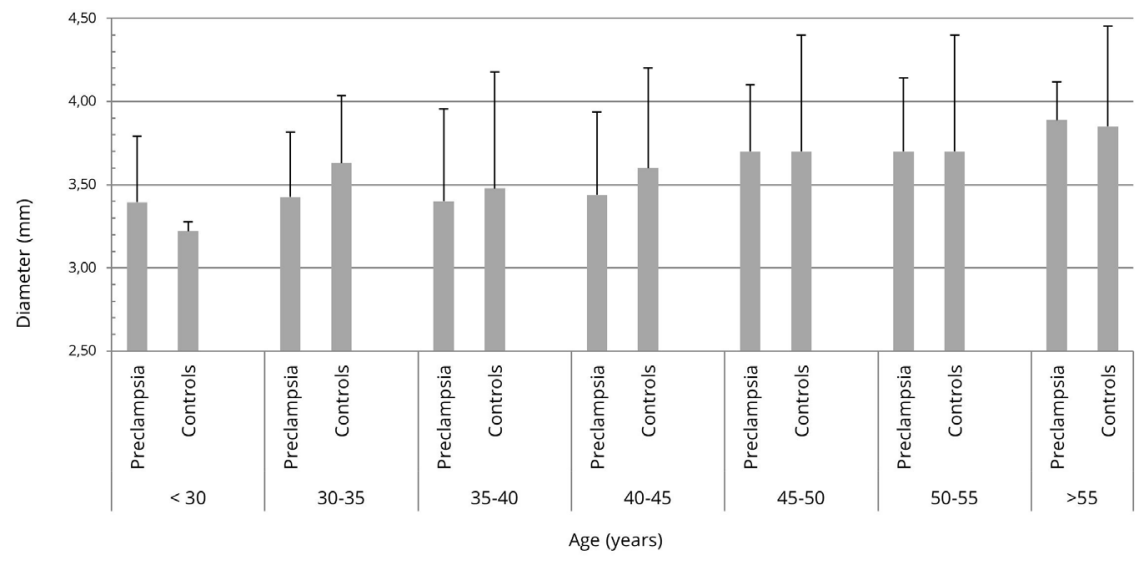

Figure 2 Baseline brachial artery diameter $(\mathrm{mm})$ in women with a history PE (Cases) and uncomplicated pregnancies (Controls) increases with increasing age, irrespective of obstetric history (Table $2 a$ and b). Data are expressed in mean and standard deviation.

Table 2b presents data of the multivariable linear regression analysis adjusted for smoking, multivitamin use, use of anti-hypertensive drugs, mean arterial pressure, fasting glucose and menopausal state. After adjustment for these factors, the difference in endothelial independent dilation measured with NG vanished while the increase in baseline diameter with age, remained $(\beta 0.011$ (0.005 - 0.016), $p<0.001)$. When corrected for factors that affect endothelial function there was a difference in group whereby women with a history of a hypertensive pregnancy had a smaller baseline diameter $(\beta-0.113$ (-0.202:-0.023), $p=0.014)$ when compared to controls. The post hoc analysis on postpartum interval did not provide different results when compared to the effect of age.

The stepwise backward elimination revealed no significant effect of potential confounders of the association between endothelial function and FMD, nor on allometrically scaled FMD. There was, besides the age effect, a significant effect of fasting glucose ( $p=0.022)$ and mean arterial pressure $(p=0.001)$ on maximal dilation through NG induced dilation. Baseline diameter was affected by age $(p<0.001)$ and mean arterial pressure $(p=0.001)$ 


\section{Unadjusted}

\section{Age}

$\begin{array}{lll} & \mathrm{B}(95 \% \mathrm{Cl}) & \text { p-value } \\ \text { Brachial artery diameter }(\mathrm{mm}) & 0.014(0.009 ; 0.019) & <0.001^{*} \\ \text { FMD (\%) } & -0.006(-.039 ; 0.028) & 0.747 \\ \text { FMD allometric (\%) } & -0.013(-0.042 ; 0.017) & 0.402 \\ \text { NG (\%) } & -0.075(-0.131 ;-0.020) & 0.008^{*}\end{array}$

Table 2a Brachial artery diameter (mm), FMD (\%), allometric flow mediated dilation (FMD allometric, $\%$ ), endothelium independent dilation measured by nitroglycerine (NG, \%) in women with a history of PE and uncomplicated pregnancies. A multivariable linear regression analysed the effect of age, group and interaction between age and group.

\section{Adjusted}

Age

\begin{tabular}{lll} 
& $B(95 \% \mathrm{Cl})$ & p-value \\
Brachial artery diameter $(\mathrm{mm})$ & $0.011(0.005 ; 0.016)$ & $<0.001$ * \\
FMD (\%) & $-0.006(-0.044 ; 0.031)$ & 0.748 \\
FMD allometric (\%) & $-0.013(-0.047 ; 0.020)$ & 0.429 \\
NG (\%) & $-0.056(-0.118 ; 0.006)$ & 0.078 \\
\hline
\end{tabular}

Table $2 \mathrm{~b}$ Brachial artery diameter ( $\mathrm{mm}$ ), FMD (\%), allometric flow mediated dilation (FMD allometric, $\%$ ), endothelium independent dilation measured by nitroglycerine (NG, \%) in women with a history of vascular complicated and uncomplicated pregnancies. A multivariable linear regression analysed the effect of age, group and interaction between age and group adjusted for smoking, multivitamin use, use of anti-hypertensive drugs, mean arterial pressure, fasting glucose and menopausal state. 
Group

B $(95 \% \mathrm{Cl})$

$-0.062(-0.149 ; 0.024)$

$-0.318(-.914 ; 0.277)$

$0.790(-0.597 ; 0.454)$

$-0.307(-.272 ; 0.659)$

\section{Age * Group}

B $(95 \% \mathrm{Cl})$

p-value

$-0.002(-0.012 ; 0.009) \quad 0.764$

$0.023(-0.051 ; 0.96)$

0.546

0.294

$0.001(-0.064 ; 0.066)$

0.981

$0.002(-0.117 ; 0.121)$

0.974

\section{Group}

B (95\%Cl)

$-0.113(-0.202 ;-0.023)$

$-0.151(-0.771 ; 0.468)$

$-0.040(-0.588 ; 0.508)$

$-0.046(-1.043 ; 0.951)$

\section{Age * Group}

B $(95 \% \mathrm{Cl})$

p-value

$\begin{array}{lll}p \text {-value } & B(95 \% \mathrm{Cl}) & p \text {-value } \\ 0.014^{*} & -0.001(-0.012 ; 0.010) & 0.829\end{array}$

0.631

$0.032(-0.042 ; 0.107)$

0.395

0.886

$0.007(-0.059 ; 0.073)$

0.827

0.928

$0.009(-0.111 ; 0.129)$

0.882 


\section{DISCUSSION}

We hypothesised that women with a hypertensive complicated pregnancy in their history have an increased age-related decline in endothelial function compared to women with uncomplicated pregnancies. In our cross sectional analysis we observed that in women between 23 and 61 years of age, endothelium-dependent vasodilation as measured by brachial FMD did not change regardless of prior hypertensive complicated pregnancy. Irrespective of obstetric history, endothelium-independent dilation as measured by NG-dilation of the brachial artery showed an age-related decline, suggesting a reduced dilatory capacity. Concurrently, brachial artery diameter showed an age-related increase which may reflect a structural basis for the age-related reduction in dilatory capacity.

The finding that baseline diameter increases with age in both groups, even when corrected for influencing factors, is in line with the diameter enlargement described by others ${ }^{24,25}$. With age, structural and functional changes occur in our arteries. The most consistent changes are diameter enlargement (dilatation) and wall thickening (remodelling), with related changes in elastic properties. The mechanisms underlying arterial aging, in particular the loss in dilatory capacity, are not fully understood. It is suggested that enlargement is a compensatory adaptation to plaque formation in order to maintain sufficient luminal area, while increases in wall thickness are instrumental to maintain wall stress/tension ${ }^{26}$. However, dilatation can also occur in the absence of plaque formation 27,28. Alternatively, dilatation appears associated with mechanical strain, occurring due to age-related loss of elastic fibres ${ }^{11,25,29}$ and/or fracturing of elastic lamellae ${ }^{30}$. Especially the age-related loss of elastin may be of particular relevance to the changes in diameter we observed. The age-related increase in diameter is not only apparent in the carotid but also in the brachial artery ${ }^{31-33}$, which is corroborated by the present study. Interestingly, this increase in baseline diameter itself is independently associated with an increased risk of $\mathrm{CVD}^{34,35}$. Since the brachial artery is hardly prone to atherosclerosis, the observed diameter increase suggests that it is more dependent on age related structural remodelling of the vessel wall rather than plaque formation.

With advancing age, elastin content of arteries decreases, elastin fibres elongate and lose some of the elastic recoil capacity. Consequently, arterial mechanical load due to blood pressure is more borne by the stiffer collagen in the arterial wall 11,36,37, at the expense of the relative dilatory capacity in response to endogenous or exogenous NO. The magnitude of (endogenous/exogenous NO-mediated) vascular dilation also depends on the ability of the smooth muscle cells to relax. 
The dilatory capacity of arteries is typically assessed by quantifying the maximum dilatory response following sublingual NG ${ }^{38}$. With aging, smooth muscle cells undergo changes that may impact the vascular dilatory capability, such as changes in phenotype and senescence 39,40. This process appears accelerated by high blood pressure ${ }^{41}$. As a result of these changes elastic vessel properties are also altered, shifting the balance from elastin towards collagen. Therefore, we interpret the age-related decline in NG-dilation we observed in the present study as reflective of an altered smooth muscle phenotype, either by loss of sensitivity to NO, stiffer acting extra-cellular matrix and/or an already stretched vessel wall due to luminal enlargement $39,42,43$.

Age-related attenuation in endothelial vascular responses in women is reported as early as from the 4th decade of life onwards, where, after menopause, these changes become more pronounced ${ }^{24,44-47}$. Within the age-range considered in this present study, namely relative young, seemingly healthy women, the observed decline in dilatory response seems primarily related to the increasing diameter along with known risk factors that negatively affect arterial function that come with age, rather than age itself 34,39,48,49. When correcting for, in particular, mean arterial pressure, brachial artery diameter differed in women with hypertensive complicated pregnancy compared to normotensive gestation, indicating a, so far unknown, vascular predisposition. A lifetime exposure to (cardiovascular) risk factors and the susceptibility of individuals to the harmful consequences of these risk factors combined with aging itself results in a decrease in arterial function ${ }^{50}$. This was apparent as the decrease in NG-induced dilation over time disappeared after adjustment for factors that affect endothelial function. However, as aging and underlying progressive risk factors for disease are interrelated, it is a challenge to separate the so called biological aging from aging associated diseases ${ }^{51}$.

Since FMD is commonly defined as the percentage increase in diameter with respect to the baseline diameter, it acts as an isometric index, suggesting the absolute (peak) diameter change is proportional to the baseline diameter. Wall shear stress homeostasis does imply that the relative diameter increase is the response of interest (DD/D), when considering the FMD stimulus (Dflow-velocity/flow-velocity) ${ }^{6}$. Some investigators argue that it is the absolute dilatory response that captures the endothelium-dependent dilatory capacity best, and hence, when using FMD as usually defined (i.e. relative changes), smaller vessels intrinsically show greater FMD ${ }^{52}$. Some studies correct for this vessel diameter dependency by allometric scaling which we chose to include in our analysis focusing on age-related changes in FMD ${ }^{23}$. We found that allometrically-scaled FMD yielded similar results compared to FMD, suggesting that differences in baseline vessel diameter did not explain the absence of an age-related decline in FMD in our study population. 
The evidence for structural and functional changes as gleaned from the observed increase in brachial artery diameter and a decrease in NG-induced dilation, were not paralleled by changes in FMD over age periods in our cohort. Several studies have shown a diminished FMD in the first decade after a vascular complicated pregnancy, while others did not find any difference in later time periods when compared to control groups ${ }^{53-59}$. In contrast to our expectations, we found an absence of difference in FMD over age periods and, based on the post hoc analysis, for postpartum interval. Studies that did find differences between younger and older patients were also cross sectional in nature whereby most did not use edge-to-edge wall tracking software, choosing to set peak diameter at a certain time point, thereby possibly overestimating differences ${ }^{60-62}$. The use of this wall tracking software, which improves reliability and reproducibility, could be viewed as a strength of our study. Another strength of this study is a longer age interval than current published studies. Moreover we analyses of both FMD and NG induced dilation which gave more insight in the age related effects of endothelial function. In addition, several factors that could confound our endothelial function measurements were taken into account in our multivariable analysis.

Limitations of this study should also be acknowledged. The study design permitted only cross-sectional analysis of age-related effects. As a result, no hypotheses on causal pathways could be tested. This may have obscured differences between cases and controls that might surface with repeated measurements within individuals. Furthermore, vascular function may only be affected by a hypertensive complicated pregnancy in a subgroup of women with a specific predisposition, which we, unfortunately could not distinguish in our study. Even though women with a vascular complicated pregnancy in their history have an increased risk of CVD the absolute incidence is low, which hampers the detection of differences in this study ${ }^{19}$. The relative young age of women in our study sample and their mostly premenopausal state may also have affected our observations. Another aspect in this apparent lack of difference in FMD between both groups is the prevalence of a positive family history for CVD. In the general population this is, depending on one's age, between $10-16 \%$ 21. In our sample we found a positive family history for CVD in about 50\% of both cases and controls. We would expect women with a history of PE to have twice the prevalence of a positive family history for CVD ${ }^{63}$. Despite that our cases seemed to be healthy at inclusion we cannot rule out that the controls that participated in our study had a different risk profile than the general population.

Lastly, FMD is considered a measurement plagued by methodological variability. However, in the hands of experienced sonographers and following a strict measurement protocol, variations can be kept to a minimum 4,64. The inter-observer agreement, quantified by intraclass correlation coefficient, is generally between 0.70 and 0.92 65-67, and the intraobserver agreement between 0.84 and 0.99 65,67; our FMD method yielded similar agreement. 


\section{CONCLUSION}

This study in young to middle aged parous women showed an age-related decline in endothelial function and structural vascular adaptation of the brachial artery. Contrary to our expectations, we could not demonstrate a difference in endothelial-dependent or -independent function, as assessed with brachial artery FMD and NG administration between subjects with and without a hypertensive obstetric history. This may indicate that the increased CV risk in women with hypertensive complicated pregnancy does not go hand in hand with a decline in endothelial function as measured by FMD or NG induced dilation. 


\section{REFERENCES}

1. Donato AJM, R. G. Walker, A. E. Lesniewski, L. A. Cellular and molecular biology of aging endothelial cells. J. Mol. Cell. Cardiol. Dec 2015;89:122-135.

2. Seals DR, Jablonski KL, Donato AJ. Aging and vascular endothelial function in humans. Clin. Sci. (Lond.). May 2011;120(9):357-375.

3. Miura H, Wachtel RE, Liu Y, et al. Flow-Induced Dilation of Human Coronary Arterioles Important Role of Ca2 -Activated K Channels Circulation. 2001;103:1992-1998.

4. Harris RA, Nishiyama SK, Wray DW, Richardson RS. Ultrasound assessment of flow-mediated dilation. Hypertension. May 2010;55(5):1075-1085.

5. Corretti MC, Anderson TJ, Benjamin EJ, et al. Guidelines for the ultrasound assessment of endothelialdependent flow-mediated vasodilation of the brachial artery. J. Am. Coll. Cardiol. 2002;39(2):257-265.

6. van Bussel FCG, van Bussel BCT, Hoeks APG, et al. A control systems approach to quantify wall shear stress normalization by flow-mediated dilation in the brachial artery. PLoS One. 2015;10(2):e0115977.

7. Inaba Y, Chen JA, Bergmann SR. Prediction of future cardiovascular outcomes by flow-mediated vasodilatation of brachial artery: a meta-analysis. Int. J. Cardiovasc. Imaging. Aug 2010;26(6):631-640.

8. Ras RT, Streppel MT, Draijer R, Zock PL. Flow-mediated dilation and cardiovascular risk prediction: a systematic review with meta-analysis. Int. J. Cardiol. Sep 20 2013;168(1):344-351.

9. Shimbo D, Grahame-Clarke C, Miyake Y, et al. The association between endothelial dysfunction and cardiovascular outcomes in a population-based multi-ethnic cohort. Atherosclerosis. May 2007;192(1):197-203.

10. Yeboah J, Folsom AR, Burke GL, et al. Predictive value of brachial flow-mediated dilation for incident cardiovascular events in a population-based study: the multi-ethnic study of atherosclerosis. Circulation. Aug 11 2009;120(6):502-509.

11. Greenwald SE. Ageing of the conduit arteries. J. Pathol. Jan 2007;211(2):157-172.

12. Harvey A, Montezano AC, Lopes RA, Rios F, Touyz RM. Vascular Fibrosis in Aging and Hypertension: Molecular Mechanisms and Clinical Implications. Can. J. Cardiol. May 2016;32(5):659-668.

13. Higashi $Y$, Kihara $Y$, Noma K. Endothelial dysfunction and hypertension in aging. Hypertens. Res. Nov 2012;35(11):1039-1047.

14. Lakatta EG. Arterial and Cardiac Aging: Major Shareholders in Cardiovascular Disease Enterprises: Part I: Aging Arteries: A "Set Up" for Vascular Disease. Circulation. 2003;107(1):139-146.

15. Aykas F, Solak Y, Erden A, et al. Persistence of cardiovascular risk factors in women with previous preeclampsia: a long-term follow-up study. J. Investig. Med. Apr 2015;63(4):641-645.

16. Hamad R, Eriksson MJ, A S, Hamsten A, Bremmea K. Decreased flow-mediated dilation is present 1 year after a pre-eclamptic pregnancy. J. Hypertens. 2007;25(2301-2307).

17. Wu P, Haththotuwa R, Shing Kwok C, et al. Preeclampsia and Future Cardiovascular Health A Systematic Review and Meta-Analysis. Circ. Cardiovasc. Qual. Outcomes. 2017.

18. Fraser A, Nelson SM, Macdonald-Wallis C, et al. Associations of pregnancy complications with calculated cardiovascular disease risk and cardiovascular risk factors in middle age: the Avon Longitudinal Study of Parents and Children. Circulation. Mar 20 2012;125(11):1367-1380.

19. McDonald SD, Malinowski A, Zhou Q, Yusuf S, Devereaux PJ. Cardiovascular sequelae of preeclampsia/ eclampsia: a systematic review and meta-analyses. Am. Heart J. Nov 2008;156(5):918-930.

20. Report of the National High Blood Pressure Education Program Working Group on High Blood Pressure in Pregnancy. Am. J. Obstet. Gynecol. 2000;183(1):s1-s22.

21. Benjamin EJ, Blaha MJ, Chiuve SE, et al. Heart Disease and Stroke Statistics-2017 Update: A Report From the American Heart Association. Circulation. Mar 7 2017;135(10):e146-e603.

22. Nederland Spr. 10 jaar perinatale registratie Nederland, de grote lijnen. 2011.

23. Atkinson G, Batterham AM. The percentage flow-mediated dilation index: a large-sample investigation of its appropriateness, potential for bias and causal nexus in vascular medicine. Vasc. Med. Dec 2013;18(6):354-365.

24. Skaug EA, Aspenes ST, Oldervoll L, et al. Age and gender differences of endothelial function in 4739 healthy adults: the HUNT3 Fitness Study. Eur J Prev Cardiol. Aug 2013;20(4):531-540.

25. van der Heijden-SpekJJ, Staessen JA, Fagard RH, Hoeks AP, Struijker Boudier HA, Van Bortel LM. Effect of Age on Brachial Artery Wall Properties Differs From the Aorta and Is Gender Dependent. Hypertension. 2000;35:637642. 
26. Schmidt-Trucksass A, Grathwohl D, Schmid A, et al. Structural, Functional, and Hemodynamic Changes of the Common Carotid Artery With Age in Male Subjects. Arterioscler. Thromb. Vasc. Biol. 1999;19:1091-1097.

27. Jensen-Urstad K, Jensen-Urstad M, Johansson J. Carotid Artery Diameter Correlates With Risk Factors for Cardiovascular Disease in a Population of 55-year-Old Subjects. Stroke. 1999;30:1572-1576.

28. Eigenbrodt ML, Bursac Z, Rose KM, et al. Common carotid arterial interadventitial distance (diameter) as an indicator of the damaging effects of age and atherosclerosis, a cross-sectional study of the Atherosclerosis Risk in Community Cohort Limited Access Data (ARICLAD), 1987-89. Cardiovasc. Ultrasound. Jan 03 2006;4:1.

29. Jacob MP. Extracellular matrix remodeling and matrix metalloproteinases in the vascular wall during aging and in pathological conditions. Biomed. Pharmacother. 2003;57(5-6):195-202.

30. O'Rourke MF, Hashimoto J. Mechanical factors in arterial aging: a clinical perspective. J. Am. Coll. Cardiol. Jul 03 2007;50(1):1-13.

31. Nair SB, Malik R, Khattar RS. Carotid intima-media thickness: ultrasound measurement, prognostic value and role in clinical practice. Postgrad. Med. J. Dec 2012;88(1046):694-699.

32. van den Munckhof I, Scholten R, Cable NT, Hopman MT, Green DJ, Thijssen DH. Impact of age and sex on carotid and peripheral arterial wall thickness in humans. Acta Physiol. (Oxf.). Dec 2012;206(4):220-228.

33. Nishiyama SK, Wray DW, Richardson RS. Aging affects vascular structure and function in a limb-specific manner. J. Appl. Physiol. 2008;105:1661-1670.

34. Holewijn S, den Heijer M, Swinkels DW, Stalenhoef AF, de Graaf J. Brachial artery diameter is related to cardiovascular risk factors and intima-media thickness. Eur. J. Clin. Invest. Jul 2009;39(7):554-560.

35. Yeboah J, Crouse JR, Hsu FC, Burke GL, Herrington DM. Brachial flow-mediated dilation predicts incident cardiovascular events in older adults: the Cardiovascular Health Study. Circulation. May 8 2007;115(18):23902397.

36. Lakatta EG. The reality of aging viewed from the arterial wall. Artery Res. Jun 01 2013;7(2):73-80.

37. Oana Mirea, Donoiu I, Plesea IE. Arterial aging a brief review. Rom. J. Morphol. Embryol. 2012;53(3):473-477.

38. Thijssen DH, Carter SE, Green DJ. Arterial structure and function in vascular ageing: are you as old as your arteries? J. Physiol. Apr 15 2016;594(8):2275-2284.

39. Montero D, Pierce GL, Stehouwer CD, Padilla J, Thijssen DH. The impact of age on vascular smooth muscle function in humans. J. Hypertens. Mar 2015;33(3):445-453; discussion 453.

40. Qiu H, Zhu Y, Sun Z, et al. Short communication: vascular smooth muscle cell stiffness as a mechanism for increased aortic stiffness with aging. Circ. Res. Sep 03 2010;107(5):615-619.

41. Mistriotis P, Andreadis ST. Vascular aging: Molecular mechanisms and potential treatments for vascular rejuvenation. Ageing Res Rev. Aug 2017;37:94-116.

42. Lacolley P, Regnault V, Nicoletti A, Li Z, Michel JB. The vascular smooth muscle cell in arterial pathology: a cell that can take on multiple roles. Cardiovasc. Res. Jul 15 2012;95(2):194-204.

43. Walsh T, Donnelly T, Lyons D. Impaired endothelial nitric oxide bioavailability: a common link between aging, hypertension, and atherogenesis? J. Am. Geriatr. Soc. Jan 2009;57(1):140-145.

44. Celermajer DS, Sorensen KE, Spiegelhalter DJ, Georgakopoulos D, Robinson J, Deanfield JE. Aging is associated with endothelial dysfunction in healthy men years before the age-related decline in women. J. Am. Coll. Cardiol. 1994;24(2):471-476.

45. Juonala M, Kahonen M, Laitinen T, et al. Effect of age and sex on carotid intima-media thickness, elasticity and brachial endothelial function in healthy adults: the cardiovascular risk in Young Finns Study. Eur. Heart J. May 2008;29(9):1198-1206.

46. Jensen-Urstad K, Johanssen J. Gender difference in age-related changes in vascular function. Journal of International Medicine. 2001(250):29-36.

47. Black MA, Cable NT, Thijssen DHJ, Green DJ. Impact of age, sex, and exercise on brachial artery flow-mediated dilatation. Am. J. Physiol. Heart Circ. Physiol. 2009;297.

48. Gerhard M, Roddy MA, Creager SJ, Creager MA. Aging Progressively Impairs Endothelium-Dependent Vasodilation in Forearm Resistance Vessels of Humans. Hypertension. 1996;27(4):849-853.

49. Gascho JA, Fanelli C, Zelis R. Aging reduces venous distensibility and the venodilatory response to nitroglycerin in normal subjects. Cardiovascular pharmacology. 1989(63):1267-1270.

50. Olsen MH, Angell SY, Asma S, et al. A call to action and a lifecourse strategy to address the global burden of raised blood pressure on current and future generations: the Lancet Commission on hypertension. The Lancet. 2016;388(10060):2665-2712.

51. Blumenthal HT. The Aging-Disease Dichotomy: True or False? J. Gerontol. 2003;58A(2):138-145. 
52. Carlson BE, Arciero JC, Secomb TW. Theoretical model of blood flow autoregulation: roles of myogenic, sheardependent, and metabolic responses. Am. J. Physiol. Heart Circ. Physiol. Oct 2008;295(4):H1572-1579.

53. Goynumer G, Yucel N, Adali E, Tan T, Baskent E, Karadag C. Vascular Risk inWomen with a History of Severe Preeclampsia. J. Clin. Ultrasound. 2012;41(3).

54. Sandvik MK, Leirgul E, Nygard O, et al. Preeclampsia in healthy women and endothelial dysfunction 10 years later. Am. J. Obstet. Gynecol. Dec 2013;209(6):569 e561-569 e510.

55. Paez O, Alfie J, Gorosito M, et al. Parallel decrease in arterial distensibility and in endothelium-dependent dilatation in young women with a history of pre-eclampsia. Clin. Exp. Hypertens. Oct 2009;31(7):544-552.

56. Lampinena KH, Ronnbackb M, Kaajaa R, Groop P-H. Impaired vascular dilatation in women with a history of pre-eclampsia. J. Hypertens. 2006;24:751-756.

57. Weissgerber TL, Milic NM, Milin-Lazovic JS, Garovic VD. Impaired Flow-Mediated Dilation Before, During, and After Preeclampsia: A Systematic Review and Meta-Analysis. Hypertension. Feb 2016;67(2):415-423.

58. Chambers JC, Fusi L, Malik IS, Haskard DO, De Swiet M, Kooner JS. Association of maternal endothelial dysfunction with preeclampsia. JAMA. Mar 28 2001;285(12):1607-1612.

59. Sandvik MK, Hallan S, Svarstad E, Vikse BE. Preeclampsia and prevalence of microalbuminuria 10 years later. Clin. J. Am. Soc. Nephrol. Jul 2013;8(7):1126-1134.

60. Irace C, Padilla J, Carallo C, Scavelli F, Gnasso A. Delayed vasodilation is associated with cardiovascular risk. Eur. J. Clin. Invest. Jun 2014;44(6):549-556.

61. Woodman RJ, Playford DA, Watts GF, et al. Improved analysis of brachial artery ultrasound using a novel edgedetection software system. Journal of Applied Fysiology. 2001;91:929-937.

62. Black MA, Cable NT, Thijssen DH, Green DJ. Importance of measuring the time course of flow-mediated dilatation in humans. Hypertension. Feb 2008;51(2):203-210.

63. Rigo J, Boze T, Derzsy Z, et al. Family history of early-onset cardiovascular disorders is associated with a higher risk of severe preeclampsia. European Journal of Obstetrics Gynecology and Reproductive Biology. Sep-Oct 2006;128(1-2):148-151.

64. van Mil AC, Greyling A, Zock PL, et al. Impact of volunteer-related and methodology-related factors on the reproducibility of brachial artery flow-mediated vasodilation: analysis of 672 individual repeated measurements. J. Hypertens. Sep 2016;34(9):1738-1745.

65. Charakida M, de Groot E, Loukogeorgakis SP, et al. Variability and reproducibility of flow-mediated dilatation in a multicentre clinical trial. Eur. HeartJ. Dec 2013;34(45):3501-3507.

66. Welsch MA, Allen JD, Geaghan JP. Stability and reproducibility of brachial artery flow-mediated dilation. Med. Sci. Sports Exerc. 2002;34(6):960-965.

67. Meirelles CdM, Leite SP, Montenegro CAB, Gomes PSC. Reliability of Brachial Artery Flow-Mediated Dilatation Measurement Using Ultrasound. Arq. Bras. Cardiol. 2007;89(3):160-167. 


\section{CHAPTER 8}

General discussion 



\section{GENERAL DISCUSSION}

This thesis provides insight on two aspects that are related to normotensive and hypertensive pregnancy. First of all it describes the adaptations a female body goes through as a result of pregnancy and discusses maladaptation resulting in a hypertensive complicated pregnancy. Secondly it contributes to current literature concerning recovery after preeclamptic pregnancy, namely long term effects on vascular endothelial function and kidney function as a result of these complicated pregnancies.

\section{ADAPTATION AND MALADAPTATION TO PREGNANCY}

The adaptive changes a female body goes through during pregnancy are extensive. A primary fall in the vascular resistance constitutes one of the earliest physiological changes in response to pregnancy ${ }^{1}$. It initiates a chain of events resulting in haemodilution, renal hyperfiltration, rise in cardiac output, higher vascular compliance and a rise in plasma volume, so as to maintain a high-flow and low-resistance circulation ${ }^{2-13}$. The mechanisms responsible for the pregnancy associated vasodilation are not yet fully understood but are thought to be mostly accomplished by a decrease of endothelial vascular responsiveness to constrictors angiotensin $\|$ and norepinephrine and an increased availability and susceptibility for endothelium- derived relaxing factor and nitric oxide (NO). Moreover relaxin, oestrogen and progesterone, which are increased during pregnancy, are also know to stimulate NO production and may account, at least partly, for the observed vascular functional adjustment ${ }^{14-17}$.

Maladaptation of maternal vascular and central hemodynamic function during pregnancy may affect remodelling of placental arteries and with it trophoblastic invasion, creating an imbalance in maternal-foetal interface. These adverse adjustments may result in additional endothelial impairment. Clinically this impairment manifests in placental dysfunction and induced foetal growth restriction on the one hand, and maternal capillary-leak induced oedema, organ hypoperfusion, hypertension, proteinuria, platelet activation and consumption and erythrocyte decay on the other hand ${ }^{18,19}$.

Pregnancy can be seen as a 'stress test' in which women with a hypertensive complicated pregnancy fail ${ }^{20}$. On the one hand adaptive responses may be attenuated leading to progressively circulatory stress and on the other hand pre-existing cardiovascular risk factors may lower the threshold towards vascular disease.

Current clinical practice is focused on detecting a hypertensive complicated pregnancy as early as possible; it does however not focus on maternal adaptation. This is partially because there is a lack of knowledge on healthy physiologic boundaries during pregnancy 
and partially because we do, as of yet, not know if potential interventions to change this adaptive process can result in reducing the risk of a hypertensive complicated pregnancy and or the maternal and foetal morbidity and mortality that coincides.

One of the aims of this thesis was to provide more insight in maternal adaptation to pregnancy and to discuss maladaptation during pregnancy. We constructed reference values for both endothelial function (flow mediated dilation (FMD)), and kidney function (glomerular filtration rate (GFR) and serum creatinine) during physiologic uncomplicated pregnancy. These reference curves are based on current literature and improve our understanding of the females' adaptive capabilities during pregnancy.

Our meta-analysis on endothelial function showed that during healthy pregnancy endothelial function, expressed by FMD of the brachial artery, changes. Both FMD and the brachial artery diameter increased as pregnancy progresses, indicating vasodilation and enhanced dilatory response. Recently a large meta-analysis reported a lower FMD before the clinical onset and during preeclampsia compared to uncomplicated pregnancies ${ }^{21}$ We only included studies using a non-pregnant reference value within the same study to compensate for different methods employed. This did exclude some studies lacking a non-pregnant reference group this and could therefore have resulted in a lower statistical power because of numbers, but increased validity because of the use of identical measurement techniques. We found that women with a complicated pregnancy did fall within the lower range of FMD values when compared to uncomplicated pregnancies. A lack of serial assessments impeded an adequate comparison of hypertensive complicated pregnancies to the course of adaptation of uncomplicated pregnancy.

However, there was, to the best of our knowledge, no comparable analysis of current literature that evaluated changes in endothelial function, measured by FMD, during pregnancy. In contrast to our expectations, FMD, as a measure to evaluate vascular function during pregnancy, maybe of limited value because of the broad confidence interval seen in the reference curve. This has partially to do with the difficulty of the measurement but also the quality of FMD measurements included in the study. Fortunately, in recent years FMD measurements protocols have become more standardised and regulated thereby improving reliability and reproducibility ${ }^{22,23}$.

Our meta-analysis on kidney function portrayed an increase in GFR during a healthy physiologic pregnancy either measured by inulin clearance or creatinine clearance compared to non-pregnant conditions. The largest increase was between 40 - 50\%, depending on the type of GFR measurement. The increase in GFR based on inulin clearance seems consistent, with similar increases between studies resulting in relative 
narrow $5^{\text {th }}$ to $95^{\text {th }}$ percentile. GFR based on creatinine clearance on the other hand differs between studies after the first trimester, leading to a wider confidence interval making the reference curve less usable in daily practice. This does not necessarily mean that the wider confidence intercal is as a result of the pregnancy but could it also be as a result of the method of collection and/or the fact that creatinine quantities in the blood can be influenced by dietary intake and/or exercise ${ }^{25}$. In contrast, serum creatinine is a stable and easy parameter to evaluate kidney function during pregnancy. Although the upper limit of the reference curve changes throughout gestation, from a clinical perspective, serum creatinine above $0.75 \mathrm{mg} / \mathrm{dl}$ ( $66 \mathrm{umol} / \mathrm{L}$ ) should be considered abnormal at all gestational ages. A serum creatinine above this threshold does not necessarily provide evidence of a hypertensive complicated pregnancy but it does give pause to look further at both maternal and foetal condition.

The use of serum creatinine-based formulas to estimate GFR over the course of pregnancy could not be analysed in this thesis which is unfortunate as it is a regularly used parameter during pregnancy. Future research measuring CKD-EPI, the more reliable estimation of GFR outside of pregnancy ${ }^{26}$, in order to establish reference values before, during and after pregnancy is relevant to clinicians in daily practice. This would improve understanding of maternal physiology during pregnancy which would also give prominence to abnormal findings.

In our meta-analysis the physiologic adaptation of kidney function as seen during uncomplicated pregnancy was not observed in women with a hypertensive complicated pregnancy. Women with a pregnancy complicated by hypertensive disease had significantly lower inulin clearance and creatinine clearance. They also had higher levels of serum creatinine when compared to uncomplicated gestation. The difference in changes of kidney function in pregnancies destined to be complicated by a gestational hypertensive disease could not be investigated because there were not enough studies in each gestational age interval.

There are some other studies performed earlier, focussing on maternal adaptation but they are scarce and all call for more research into this particular field of medicine ${ }^{27-29}$. By providing more insight in the normal physiology during pregnancy, the understanding of pathological changes during pregnancy increases and can possibly facilitate in a way to distinguish between both. We therefore investigated maternal adaptation in a small observation study in women with a hypertensive complicated pregnancy in their history. We found that in women destined to develop a recurrent hypertensive disorder in their next pregnancy, the initial maternal renal and hemodynamic adaptive response to pregnancy was attenuated when compared to that in their counterparts who had an uneventful subsequent 
pregnancy. The attenuated response consisted of a smaller rise in creatinine clearance and cardiac output. This maladaptive response is interesting to highlight because it might be able to provide the opportunity to use maternal cardiovascular maladaptation as a screening method to select women at risk for hypertensive complicated pregnancies. Even though our study focused on a high risk population, making it more likely to find maladaptation in a subsequent pregnancy, it does provide an interesting window into maladaptive responses to pregnancy and should instigate more research on maternal adaptation to pregnancy.

\section{LONG TERM EFFECTS RESULTING FROM A HYPERTENSIVE COMPLICATED PREGNANCY}

Over the past decade a hypertensive complicated pregnancy, in particular preeclampsia, has become a well-accepted risk factor for the development of future cardiovascular disease (CVD). Several prospective studies have reported that the future risk of CVD after preeclampsia is both related to the severity of the disease and some also report association with the number of times it occurs during a lifetime ${ }^{30,31}$. Even though women before menopause have relative lower risk of developing CVD of around 2\%, this risk does increase with age ${ }^{32,33}$. The absolute incidence coincides with a 2 to 7 fold increase in relative risk of CVD after preeclampsia, depending on the severity of the disease during pregnancy ${ }^{31,33}$. These figures are comparable to women with type 1 diabetes.

Women with a hypertensive complicated pregnancy in their history also have a 4 to 5 times higher risk of end stage renal disease ${ }^{34}$, although the absolute increase is relatively low, due to the incidence of $0.0037 \%$. Chronic kidney disease (CKD), a precursor to end stage renal disease, can still be, even in severe cases, reversible. This can be either spontaneously or with treatment, therefore making it an important disease to investigate, especially in relation to preeclampsia. Moreover, a two-fold increase in albuminuria, that can be present at any stage of CKD, is associated with $29 \%$ more risk for cardiovascular mortality ${ }^{35}$. As the risk of CVD is also increased in women with CKD, we could extrapolate that women with a history of a hypertensive disease and CKD have an even more increased risk of CVD ${ }^{36}$.

Because of these reasons we chose to investigate the occurrence of CKD in a cohort of women after hypertensive complicated pregnancy by applying the KDIGO criteria. The KDIGO guideline, of the international society of nephrology, was written for the general patient population and indicates that kidney disease persisting for more than four months is considered to be chronic, irrespective of aetiology. This guideline provided us with guidance on how often we should follow up on women with a history of preeclampsia after delivery to ensure that microalbuminuria would regress over time and to clarify which women should be further investigated for kidney disease. Following this guideline, about $14 \%$ of women after hypertensive complicated pregnancy should have at least yearly monitoring of their kidney function, mainly due to moderately increased microalbuminuria 
found around one year after giving birth. Only $1.4 \%$ of our population was classified to be at high risk for kidney function deterioration and would be in need of referral to specialist care, preferably a nephrologist. It was the first study to use the KDIGO criteria in women with a hypertensive complicated pregnancy it their history, and provides an opportunity to find women at risk of CKD and advice on necessary follow up.

As women with a history of a hypertensive complicated pregnancy can exhibit endothelial dysfunction we also postulated that there would be a relationship between vascular endothelial function expressed by FMD and kidney function expressed by GFR and microalbuminuria. This study demonstrated that, five years after pregnancy, a decreased endothelial function is present, expressed by a decreased FMD in women with a hypertensive complicated pregnancy in their history compared to women with uncomplicated pregnancies. There was however not a decrease in kidney function and no apparent correlation between the two. Even though decreased FMD and microalbuminuria both reflect endothelial dysfunction they may reflect different aspects of endothelial function. Alternatively endothelial function as assessed by microalbuminuria may follow a different recovery rate than endothelial function expressed by FMD.

Long term effects on vascular endothelial function after hypertensive complicated pregnancy are also affected by the progression of time. Independent of sex, with increasing age, several changes to arteries contribute to the increased risk of CVD. The most consistent changes are diameter enlargement (dilatation) and wall thickening (remodelling), with related changes in elastic properties. The mechanisms underlying arterial aging, in particular the loss in dilatory capacity, are not fully understood. It is suggested that enlargement is a compensatory adaptation to plaque formation in order to maintain sufficient luminal area, while increases in wall thickness are instrumental to maintain wall stress/tension ${ }^{37}$. However, enlargement can also occur in the absence of plaque formation ${ }^{38,39}$. Alternatively, enlargement appears to be associated with mechanical strain, occurring due to age-related loss of elastic fibres ${ }^{40-42}$ and/or fracturing of elastic lamellae ${ }^{43}$. One of the most clinically important changes in the development of vascular endothelial dysfunction is the impaired possibility to vasodilate.

We found that independent of obstetric history, FMD did not change over time periods while, consistent with vasculature stiffening, maximum vessel dilatory capacity (measured by nitroglycerine induced dilation) decreased and vessel diameter increased. Factors that affect endothelial function explained the age-related vessel stiffening observed. The observed increase in vessel diameter with aging did reamain, also after adjustment for these factors. Of clinical importance is the increase in baseline diameter itself that is also independently associated with an increased risk of CVD 44,45. 
The absence of difference in FMD between women with a history of preeclampsia and controls after uncomplicated pregnancy was contradictory to our previous study that did show a decreased FMD several years after a hypertensive complicated pregnancy ${ }^{46}$. This could be partially due to selection bias seeing as the previous study included women that were willing to also participate in a rigorous exercise program to improve health and the latter study saw more women that were to benefit from a cardiovascular evaluation mostly due to a familial cardiovascular burden. Differences between study populations in relation to the severity of the disease during pregnancy could also play a role. Interestingly FMD appears to be diminished in several but not all studies in women with a history of a preeclampsia ${ }^{47,48}$. This uncovers several difficulties: the heterogeneity of the disease studied, selection in studies population and reliability of the used measurement, thereby make it difficult to compare study results.

Age-related attenuation of endothelial vascular response in women, including a decrease in FMD, is reported as early as from the $4^{\text {th }}$ decade of life onwards, where after menopause, these changes become more pronounced ${ }^{49-53}$. A lifetime exposure to cardiovascular risk factors and the susceptibility of individuals to the harmful consequences of these risk factors combined with aging itself is what results in a decrease in arterial function ${ }^{54}$. Surprisingly, we did not find an age related decrease in FMD in our young to middle aged controls nor in women without a history of preeclampsia. We did however find a decrease in maximal dilation capacity and increase in vessel diameter in both groups, both indicative of age related changes.

FUTURE PERSPECTIVES FOR WOMEN WITH HYPERTENSIVE COMPLICATED PREGNANCY IN THEIR HISTORY

Preeclampsia is a cardiovascular insult at a young age which may provide us opportunities for early prevention strategies to reduce risks. However, despite the increased relative risk the majority will not develop CVD and/ or CKD. An ultimate goal would be to be able to screen women after a hypertensive complicated pregnancy and to select a subgroup of women at risk of CVD and/or CKD. These women could then be subject to preventive measures that can postpone or even prevent progression of disease. Although feasible this is still a distant goal because there is much information lacking about treatment, adherence, effectiveness and costs of such strategies within this group of apparently healthy young women.

Classical cardiovascular risk factors are important players in the development of a dysfunctioning endothelium and consequently CVD. A worldwide study in 52 countries showed that 9 (potentially) modifiable risk factors account for over $90 \%$ of the population attributable risk of a first myocardial infarction. These 9 aspects are: smoking, dyslipidaemia, 
hypertension, diabetes mellitus, abdominal obesity, psychosocial factors, daily consumption of fruits and vegetables, regular alcohol consumption and regular physical activity ${ }^{55}$. The attribution of these risk factors to CVD seems even higher in women than in men. This thesis did not focus on cardiovascular risk factors, we are however aware that there are studies that indicate that women with a history of preeclampsia have a higher incidence of these modifiable risk factors, even before pregnancy ${ }^{56,57}$.

The Framingham risk score uses age, total and high-density lipoprotein cholesterol, systolic blood pressure, treatment for hypertension, smoking and diabetes status to calculate the risk of future CVD. It does take sex into account but a history of a hypertensive complicated pregnancy is not included in this traditional risk model. Interestingly, some studies calculated Framingham risk scores for women with a history of preeclampsia and found either a higher prevalence of risk factors or a higher risk of CVD compared to women with normotensive pregnancies in their history 58,59 .

Luckily, in recent years guidelines have been adapted to address CVD and CVD prevention in women ${ }^{60-62}$. These guidelines do acknowledge the increased risk for CVD after a hypertensive complicated pregnancy but do not offer specific recommendations on frequency or content of CVD risk assessment. General recommendations however are made, aimed at blood pressure control, lifestyle advice and exercise. The American Heart association recommends, for the general population, taking your blood pressure once every two years and cholesterol tests every 5 years above the age of 20 .

Blood pressure regulation, as part of cardiovascular risk management, improves the prognosis of both CVD and CKD ${ }^{63}$. Three studies in this thesis show a significant increased, though still considered normal, blood pressure and/or more use of antihypertensive drugs in women with a history of a hypertensive complicated pregnancy when compared to controls. Appropriate blood pressure regulation seems therefore particularly relevant for women with a hypertensive complicated pregnancy in their history. Besides, blood pressure regulation and kidney function screening by measuring albuminuria has proven to be not only effective but also cost-effective, when taking possible treatment with ACE inhibitors into account, as it reduces the risk of both CKD and CVD ${ }^{64}$. This makes screening for albuminuria in a population of women with a history of preeclampsia even more plausible.

Based on this thesis and from a practical point of view we would advise evaluation of kidney function, for women with a history of preeclampsia, at the regular 6 weeks postpartum control. Women who then have microalbuminuria could be seen one year thereafter by their general physician for follow up of kidney function and a cardiovascular (risk factor) 
assessment. A cardiovascular risk assessment for women with a hypertensive complicated pregnancy in their history that is focused on aformentioned (potentially) modifiable risk factors combined with kidney function assessment, and management thereof, could be performed every 3 years by their general physician thereby keeping track of women at higher risk of both CVD and CKD.

\section{FUTURE RESEARCH}

Focussing on maternal adaptation during an uncomplicated pregnancy would be valuable with the thought in mind that maladaptation could be better discernible when knowing more about physiology during pregnancy. Serial measurements of, for example, mean arterial pressure, cardiac output, kidney function and peripheral resistance in women with uncomplicated pregnancies and pregnancies complicated by a hypertensive disease would be prized information. This could eventually lead us to be able to discern possible differences in patterns of adaptation in which some women develop a (more severe) hypertensive complication compared to other that do not, providing us guidance in frequency of follow-up. This could ultimately lead to research focussing on possible interventions aimed at adjusting maladaptation, early in pregnancy, if this proves to reduce maternal and foetal complications.

A major problem in the prevention of CVD has been the difficulty to identify individuals at risk at an early enough stage for them to benefit from interventions. Research focussed on identifying those women, after hypertensive complicated pregnancy, that are at risk of CVD would be relevant as these women would develop CVD earlier that they counterparts. By serial assessment of cardiovascular risk factors in women after a hypertensive complicated pregnancy, insight could be obtained in the pattern of recovery and possibly single out women at higher risk. The goal to strive for would be to search for a way to predict which women would develop CVD and/or CKD after a hypertensive complicated pregnancy. 


\section{REFERENCES}

1. Conrad KP. Mechanisms of Renal Vasodilatation and Hyperfiltration During Pregnancy: Current Perspectives and Potentia; Implications for Preeclampsia. Endothelium. 2005 2005;12:57-62.

2. Mahendru AA, Everett TR, Wilkinson IB, Lees CC, McEniery CM. A longitudinal study of maternal cardiovascular function from preconception to the postpartum period. J. Hypertens. Apr 2014;32(4):849-856.

3. ClappJF, Capeless E. Cardiovascular Function Before, During, and After the First and Subsequent Pregnancies. Am. J. Cardiol. 1997;80:1469-1473.

4. Grindheim G, Estensen ME, Langesaeter E, Rosseland LA, Toska K. Changes in blood pressure during healthy pregnancy: a longitudinal cohort study. J. Hypertens. Feb 2012;30(2):342-350.

5. deAlvarez RR, Wash S. Glomerular filtration rates, renal plasma flow, and sodium and water retention in pregnancy toxemia. Am. J. Obstetrics and Gynaecology. Dec 1950;60(5):1051-1067.

6. Pritchard JA. Changes in the blood volume during pregnancy and delivery. Anesthesiology. 1965;26(4):393-399.

7. Chesley LC. Plasma and red cell volumes during pregnancy. Am. J. Obstet. Gynecol. Feb 01 1972;112(3):440-450.

8. Bader RA, Bader ME, Rose DF, Braunwald E. Hemodynamics at rest and during exercise in normal pregnancy as studies by cardiac catheterization. J. Clin. Invest. Oct 1955;34(10):1524-1536.

9. Stephen C. Robson, Stewart Hunter, Boys RJ, Dunlop W. Serial study of factors influencing changes in cardiac output during human pregnancy. Am. J. Physiol. 1989;256(25):1060-1065.

10. Hunter S, Robson SC. Adaptation of the maternal heart in pregnancy. Br. Heart J. 1992;68:540543.

11. Mabie WC, DiSessa TG, Crocker LG, Sibai BM, Arheart KL. A longitudinal study of cardiac output in normal human pregnancy. Am. J. Obstet. Gynecol. Mar 1994;170(3):849-856.

12. Duvekot JJ, Cheriex EC, Pieters FA, Menheere PP, Peeters LL. Early pregnancy changes in hemodynamics and volume homeostasis are consecutive adjustments triggered by a primary fall in systemic vascular tone. Am. J. Obstet. Gynecol. Dec 1993;169(6):1382-1392.

13. Duvekot JJ, Peeters LLH. Maternal cardiovascular hemodynamic adaptation to pregnancy. Obstet. Gynecol. Surv. Dec 1994;49(12 Suppl):S1-14.

14. Haynes MP, Sinha D, Russell KS, et al. Membrane Estrogen Receptor Engagement Activates Endothelial Nitric Oxide Synthase via the PI3-Kinase-Akt Pathway in Human Endothelial Cells. Circ Res. . 2000;87:677-682.

15. Simoncini T, Hafezi-Moghadam A, Brazil DP, Ley K, Chin WW, Liao JK. Interaction of oestrogen receptor with the regulatory subunit of phosphatidylinositol-3-OH kinase. Nature. Sep 28 2000;407(6803):538-541.

16. Boeldt DS, Bird IM. Vascular adaptation in pregnancy and endothelial dysfunction in preeclampsia. J. Endocrinol. Jan 2017;232(1):R27-R44.

17. Bird IM, Zhang L, Magness RR. Possible mechanisms underlying pregnancy-induced changes in uterine artery endothelial function. Am. J. Physiol. Regul. Integr. Comp. Physiol. Feb 2003;284(2):R245-258.

18. Stella CL, Sibai BM. Preeclampsia: Diagnosis and management of the atypical presentation. J. Matern. Fetal Neonatal Med. Jul 2006;19(7):381-386.

19. Report of the National High Blood Pressure Education Program Working Group on High Blood Pressure in Pregnancy. Am. J. Obstet. Gynecol. 2000;183(1):s1-s22.

20. Naveed Sattar IAG. Pregnancy complications and maternal cardiovascular risk: opportunities for intervention and screening? Br. Med. J. 2002;325:157-160.

21. Weissgerber TL, Milic NM, Milin-Lazovic JS, Garovic VD. Impaired Flow-Mediated Dilation Before, During, and After Preeclampsia A Systematic Review and Meta-Analysis. Hypertension. Feb 2016;67(2):415-423.

22. Corretti MC, Anderson TJ, Benjamin EJ, et al. Guidelines for the ultrasound assessment of endothelial-dependent flow-mediated vasodilation of the brachial artery. J. Am. Coll. Cardiol. 2002;39(2):257-265. 
23. Foo FL, McEniery CM, Lees C, Khalil A, International Working Group on Maternal H. Assessment of arterial function in pregnancy: recommendations of the International Working Group on Maternal Haemodynamics. Ultrasound Obstet. Gynecol. Jul 012017.

24. Lindheimer MD, Davison JM, Katz Al. The kidney and hypertension in pregnancy: twenty exciting years. Semin. Nephrol. Mar 2001;21(2):173-189.

25. Stevens LA, Coresh J, Greene T, Levey AS. Assessing kidney function--measured and estimated glomerular filtration rate. N. Engl. J. Med. Jun 8 2006;354(23):2473-2483.

26. Inker LA, Shaffi K, Levey AS. Estimating glomerular filtration rate using the chronic kidney diseaseepidemiology collaboration creatinine equation: better risk predictions. Circ. Heart Fail. May 01 2012;5(3):303-306.

27. Melchiorre K, Sharma R, Khalil A, Thilaganathan B. Maternal Cardiovascular Function in Normal Pregnancy: Evidence of Maladaptation to Chronic Volume Overload. Hypertension. Apr 2016;67(4):754-762.

28. Tiralongo GM, Lo Presti D, Pisani I, et al. Assessment of total vascular resistance and total body water in normotensive women during the first trimester of pregnancy. A key for the prevention of preeclampsia. Pregnancy Hypertens. Apr 2015;5(2):193-197.

29. Bosio PM, McKenna PJ, Conroy R, O'Herlihy C. Maternal Central Hemodynamics in Hypertensive Disorders of Pregnancy. Obstatrics \& Gynecology. 1999;94:978-984.

30. Lee G, Tubby J. Preeclampsia and the risk of cardiovascular disease later in life--A review of the evidence. Midwifery. Dec 2015;31(12):1127-1134.

31. Bellamy L, Casas JP, Hingorani AD, Williams DJ. Pre-eclampsia and risk of cardiovascular disease and cancer in later life: systematic review and meta-analysis. Br. Med.J. Nov10 2007;335(7627):974.

32. Leslie MS, Briggs LA. Preeclampsia and the Risk of Future Vascular Disease and Mortality: A Review. J Midwifery Womens Health. May 2016;61(3):315-324.

33. McDonald SD, Malinowski A, Zhou Q, Yusuf S, Devereaux PJ. Cardiovascular sequelae of preeclampsia/eclampsia: a systematic review and meta-analyses. Am. HeartJ. Nov 2008;156(5):918930.

34. Bjørn Egil Vikse, Lorentz M. Irgens, Torbjørn Leivestad, Rolv Skjærven, Iversen BM. Preeclampsia and the Risk of End-Stage Renal Disease. N. Engl. J. Med. 2008;359:800-809.

35. Hillege HL. Urinary Albumin Excretion Predicts Cardiovascular and Noncardiovascular Mortality in General Population. Circulation. 2002;106(14):1777-1782.

36. Kurth T, de Jong PE, Cook NR, Buring JE, Ridker PM. Kidney function and risk of cardiovascular disease and mortality in women: a prospective cohort study. Br. Med. J. Jun 29 2009;338:b2392.

37. Schmidt-Trucksass A, Grathwohl D, Schmid A, et al. Structural, Functional, and Hemodynamic Changes of the Common Carotid Artery With Age in Male Subjects. Arterioscler. Thromb. Vasc. Biol. 1999;19:1091-1097.

38. Jensen-Urstad K, Jensen-Urstad M, Johansson J. Carotid Artery Diameter Correlates With Risk Factors for Cardiovascular Disease in a Population of 55-year-Old Subjects. Stroke. 1999;30:15721576.

39. Eigenbrodt ML, Bursac Z, Rose KM, et al. Common carotid arterial interadventitial distance (diameter) as an indicator of the damaging effects of age and atherosclerosis, a cross-sectional study of the Atherosclerosis Risk in Community Cohort Limited Access Data (ARICLAD), 1987-89. Cardiovasc. Ultrasound. Jan 03 2006;4:1.

40. Greenwald SE. Ageing of the conduit arteries. J. Pathol. Jan 2007;211(2):157-172.

41. Jacob MP. Extracellular matrix remodeling and matrix metalloproteinases in the vascular wall during aging and in pathological conditions. Biomed. Pharmacother. 2003;57(5-6):195-202.

42. van der Heijden-Spek JJ, Staessen JA, Fagard RH, Hoeks AP, Struijker Boudier HA, Van Bortel LM. Effect of Age on Brachial Artery Wall Properties Differs From the Aorta and Is Gender Dependent. Hypertension. 2000;35:637-642.

43. O'Rourke MF, Hashimoto J. Mechanical factors in arterial aging: a clinical perspective. J. Am. Coll. Cardiol. Jul 03 2007;50(1):1-13.

44. Witte DR, Westerink J, de Koning EJ, van der Graaf Y, Grobbee DE, Bots ML. Is the association between flow-mediated dilation and cardiovascular risk limited to low-risk populations? J. Am. Coll. Cardiol. Jun 21 2005;45(12):1987-1993. 
45. Daichi Shimboa CG-C, Yumiko Miyake a, Carlos Rodriguez,Robert Sciacca, Marco Di Tullio, Bernadette Boden-Albala,, Ralph Sacco SH. The association between endothelial dysfunction and cardiovascular outcomes in a population-based multi-ethnic cohort. Atherosclerosis. 2006;192:197-203.

46. Lopes van Balen VA, Spaan JJ, Cornelis T, Heidema WM, Scholten RR, Spaanderman MEA. Endothelial and kidney function in women with a history of preeclampsia and healthy parous controls: A case control study. Microvasc. Res. Mar 2018;116:71-76.

47. Chambers JC, Fusi L, Malik IS, Haskard DO, De Swiet M, Kooner JS. Association of maternal endothelial dysfunction with preeclampsia. JAMA. Mar 28 2001;285(12):1607-1612.

48. Sandvik MK, Hallan S, Svarstad E, Vikse BE. Preeclampsia and prevalence of microalbuminuria 10 years later. Clin. J. Am. Soc. Nephrol. Jul 2013;8(7):1126-1134.

49. Celermajer DS, Sorensen KE, Spiegelhalter DJ, Georgakopoulos D, Robinson J, Deanfield JE. Aging is associated with endothelial dysfunction in healthy men years before the age-related decline in women. J. Am. Coll. Cardiol. 1994;24(2):471-476.

50. Juonala M, Kahonen M, Laitinen T, et al. Effect of age and sex on carotid intima-media thickness, elasticity and brachial endothelial function in healthy adults: the cardiovascular risk in Young Finns Study. Eur. Heart J. May 2008;29(9):1198-1206.

51. Jensen-Urstad K, Johanssen J. Gender difference in age-related changes in vascular function. Journal of International Medicine. 2001(250):29-36.

52. Black MA, Cable NT, Thijssen DHJ, Green DJ. Impact of age, sex, and exercise on brachial artery flow-mediated dilatation. Am. J. Physiol. Heart Circ. Physiol. 2009;297.

53. Skaug EA, Aspenes ST, Oldervoll L, et al. Age and gender differences of endothelial function in 4739 healthy adults: the HUNT3 Fitness Study. Eur J Prev Cardiol. Aug 2013;20(4):531-540.

54. Olsen MH, Angell SY, Asma S, et al. A call to action and a lifecourse strategy to address the global burden of raised blood pressure on current and future generations: the Lancet Commission on hypertension. The Lancet. 2016;388(10060):2665-2712.

55. Yusuf S, Hawken S, Ôunpuu S, et al. Effect of potentially modifiable risk factors associated with myocardial infarction in 52 countries (the INTERHEART study): case-control study. The Lancet. 2004;364(9438):937-952.

56. Udenze IC. Association of pre-eclampsia with metabolic syndrome and increased risk of cardiovascular disease in women: A systemic review. Niger. J. Clin. Pract. Jul-Aug 2016;19(4):431435.

57. Veerbeek JH, Hermes W, Breimer AY, et al. Cardiovascular disease risk factors after early-onset preeclampsia, late-onset preeclampsia, and pregnancy-induced hypertension. Hypertension. Mar 2015;65(3):600-606.

58. van Rijn BB, Nijdam ME, Bruinse HW, et al. Cardiovascular disease risk factors in women with a history of early-onset preeclampsia. Obstet. Gynecol. May 2013;121(5):1040-1048.

59. Fraser A, Nelson SM, Macdonald-Wallis C, et al. Associations of pregnancy complications with calculated cardiovascular disease risk and cardiovascular risk factors in middle age: the Avon Longitudinal Study of Parents and Children. Circulation. Mar 20 2012;125(11):1367-1380.

60. Bushnell C, McCullough LD, Awad IA, et al. Guidelines for the prevention of stroke in women: a statement for healthcare professionals from the American Heart Association/American Stroke Association. Stroke. May 2014;45(5):1545-1588.

61. Heida KY, Bots ML, de Groot CJ, et al. Cardiovascular risk management after reproductive and pregnancy-related disorders: A Dutch multidisciplinary evidence-based guideline. Eur J Prev Cardiol. Nov 2016;23(17):1863-1879.

62. Mosca L, Benjamin EJ, Berra K, et al. Effectiveness-based guidelines for the prevention of cardiovascular disease in women--2011 update: a guideline from the american heart association. Circulation. Mar 22 2011;123(11):1243-1262.

63. Parati G, Ochoa JE, Bilo G. Blood pressure variability, cardiovascular risk, and risk for renal disease progression. Curr. Hypertens. Rep. Oct 2012;14(5):421-431.

64. Boersma C, Gansevoort R, Pechlivanoglou P, et al. Screen-and-Treat Strategies for Albuminuria to Prevent Cardiovascular and Renal Disease: Cost-Effectiveness of Nationwide and Targeted Interventions Based on Analysis of Cohort Data From the Netherlands. Clin. Ther. 2010;32(6):11031121. 


\section{CHAPTER 9}

Valorisation 



\section{VALORISATION}

This chapter addresses the relevance and possible impact of this thesis for society. In this thesis the objective was to provide more insight in maternal adaptation to pregnancy. Thereafter we discuss maladaptation during pregnancy and bring attention to possible long term consequences on vascular endothelial and kidney function as a result of a hypertensive complicated pregnancy.

\section{SOCIETAL RELEVANCE}

There are half a million maternal deaths worldwide related to pregnancy, of which 1\% occurs in high income countries ${ }^{1}$. Preeclampsia, a hypertensive complicated pregnancy, occurs in 2 - 8\% of all pregnancies. Interestingly, the proportion of maternal death directly related to preeclampsia is similar in high income compared to middle and low income countries. In the Netherlands, preeclampsia is the leading cause of maternal mortality 2. It impacts maternal health and child's health and wellbeing, especially if the child is born prematurely. For many women, developing preeclampsia is generally an unexpected, difficult and traumatic life experience. This is particularly relevant if they felt ill, gave birth too early, or if their baby died. It is not surprising that preeclampsia, especially with secondary neonatal intensive care necessity, strongly links to post traumatic stress disorder and depression. Moreover, in presence or absence of psychological trauma, women often find difficulties to reintegrate in societal labour after giving birth.

Maternal cardiovascular adaptation during pregnancy can be suboptimal contributing in the development of a hypertensive complicated pregnancy. In conjunction with maternal characteristics, the placenta plays a pivotal role in the development of such a maladaptive pregnancy. During the disease state however, there are currently no curative treatment options available, but only symptomatic treatment to correct the underlying cardiovascular shortcomings, namely optimizing blood-pressure and -flow and protection from possible imminent seizures that can occur as a result of the disease.

Better understanding of the healthy adaptational process during pregnancy may result in future therapies targeted to improve maternal cardiovascular adjustments during the implantation and development of the placenta and possibly reduce penetrance of parallel maternal circulatory maladaptation. Consequently, the risk of later maternal and foetal complications may be reduced.

Adaptational changes supporting physiological pregnancy are not systematically taken into account. In order to facilitate nomogram guided care, we established reference values for both vascular endothelial function, expressed by flow mediated dilation, and kidney 
function, expressed by glomerular filtration rate, to describe the normal physiological processes during pregnancy. These reference curves are based on current literature and improve our understanding of the females' adaptive capabilities during pregnancy. In these meta-analyses we highlight the maladaptive response in women with a hypertensive complicated pregnancy. Moreover, we set up an explorative study from which we concluded that women destined to develop a hypertensive complicated pregnancy exhibited a decreased adaptation of both cardiac and kidney function before the occurrence of disease. This indicates the possibility of using maternal haemodynamic maladaptation as an indication for an increased risk of a hypertensive complicated pregnancy. A nonphysiological adaptive pattern detailed by serial measurements may therefore open up the possibility to institute tailored preventive measures before disease becomes apparent.

Recently the possible long term consequences of a hypertensive complicated pregnancy have been more in the scientific spotlight. The increased risk of cardiovascular and chronic kidney disease in these affected women have become more apparent, more accepted in medicine and integrated in guidelines. Several meta-analyses show this increased risk. Women with a hypertensive complicated pregnancy are at a 2 - 6 fold increased risk of future cardiovascular disease and a 3 - 7 fold increased risk of future kidney disease, depending on the severity of the disease hypertensive disease during pregnancy ${ }^{3,4}$. It seems therefore relevant to understand why this increased risk is present and what, if any, the common pathways would be. The understanding of the system-biology towards disease might eventually provide possibilities to develop targeted therapies.

Three chapters in this thesis are dedicated to this particular understanding of possible correlations between a hypertensive complicated disease and cardiovascular and/or chronic kidney disease in later life. We brought attention to long term consequences on both vascular endothelial and kidney function and found that women with a history of preeclampsia have a decreased kidney function, expressed by loss of albumins in their urine. This indicates that a particular subset of woman is at risk of future kidney disease.

We also found that aging women independently of a hypertensive complicated pregnancy have stiffened brachial arteries along with increased vessel diameter, even though endothelial dependent vasodilation capacity remained intact. Age related arterial stiffening primarily seems related to emerging traditional cardiovascular risk factors and only partially to aging itself. This finding sheds a different light on the concept of vascular aging, as aging cannot be influenced, while the occurrence and development of traditional cardiovascular risk factors can. 
A major problem in the prevention of cardiovascular disease has been the difficulty to identify individuals at risks at an early enough stage for them to benefit from interventions. Appropriate treatment, aimed at preventing progression of cardiovascular disease and risk factors, is important since blood pressure control, lifestyle advice and exercise can slow down or even reverse progression of cardiovascular disease. The current approach in the follow-up of women with a history of a hypertensive complicated pregnancy is not uniform or even, in most cases, recommended in healthcare communities. However, these women are at an increased risk and should therefore be monitored more closely. Changes in lifestyle and a healthy exercise pattern can be beneficial not only for the women with an increased risk but could also stimulate the whole family to lead a healthier life. A healthy life style is also beneficial for general health and can prevent or reduce the risk of other diseases as well.

A large cross-sectional study is still ongoing (titled Queen of Hearts) with the aim to improve diagnosis of early stages of cardiovascular disease in women, in the particular subset of women with a hypertensive pregnancy in their history. The main goal is to obtain biomarkers, based on pathogenesis of microvascular disease, which improve the early diagnosis of diastolic dysfunction and heart failure in women.

This thesis aimed to highlight the importance of a better understanding of the maternal adaptations during pregnancy and the possible long term effect of a hypertensive complicated pregnancy. By understanding the physiological adaptation to pregnancy we may eventually be able to reduce maladaptation leading to a hypertensieve complicated pregnancy and to improve maternal health. More research is needed not only to improve understanding of adaptation but also to evaluate effectiveness and costs of such strategies.

\section{DISSEMINATION AND TARGET}

This thesis highlights a patient population that are young women, who are generally in good health except for a hypertensive complicated pregnancy in their history and are curious to know how this disease affects their cardiovascular risk. We have an obligation as physicians to inform women of this increased risk and to provide guidance on how to prevent and/or treat women affected. Recent changes in guidelines do help in the dissemination of this information to physicians and eventually to patients. 
We have taken various efforts to ensure that the knowledge gained from our studies is spread across different target groups. Four out of six studies, described in this dissertation, have already been published in several international journals to spread the findings to the international research community. In addition, most findings have been presented at national and international conferences that hosted many researchers from across the globe. The findings discussed in this dissertation are relevant to multiple target groups. Firstly, researchers in the field of obstetrics, nephrology and general practitioners can benefit from the information in this thesis. Secondly, physicians may use nomograms to follow healthy pregnancy adjustments form adverse patterns. 


\section{REFERENCES}

1. Duley L. The global impact of pre-eclampsia and eclampsia. Semin. Perinatol. Jun 2009;33(3):130-137.

2. Schutte JM, Steegers EA, Schuitemaker NW, et al. Rise in maternal mortality in the Netherlands. BJOG. Mar 2010;117(4):399-406.

3. Bjørn Egil Vikse, Lorentz M. Irgens, Torbjørn Leivestad, Rolv Skjærven, Iversen BM. Preeclampsia and the Risk of End-Stage Renal Disease. N. Engl. J. Med. 2008;359:800-809.

4. McDonald SD, Malinowski A, Zhou Q, Yusuf S, Devereaux PJ. Cardiovascular sequelae of preeclampsia/ eclampsia: a systematic review and meta-analyses. Am. Heart J. Nov 2008;156(5):918-930. 


\section{CHAPTER 10}

Summary/Samenvatting 



\section{SUMMARY}

Preeclampsia is a syndrome occurring during pregnancy that is a state of endothelial dysfunction, expressed by hypertension and commonly coincided by loss of proteins in the urine, disseminated intravascular coagulation, hepatic and kidney problems. During preeclampsia vascular endothelial function is disturbed. This can be seen by a decrease or downregulations of different factors needed for uncomplicated maternal adaptation to pregnancy. The changes a female body goes through during pregnancy are extensive. This thesis contains research providing more insight in maternal adaptation during pregnancy, necessary to understand normal pattern and be able to distinguish proper adaptation from maladaptation.

Long term effects of preeclampsia are an increased risk of cardiovascular and chronic kidney disease. This thesis contributes to current literature concerning changes that are present after a hypertensive disease during pregnancy. This was conducted by analysing long term effects on vascular endothelial function and kidney function as a result of a hypertensive complicated pregnancy, namely preeclampsia.

CHAPTER 1 provides a general introduction as well as the aims and outline of this thesis.

CHAPTER 2 is a review and meta-analysis with the purpose to establish reference values for flow mediated dilation (FMD) and brachial artery diameter in pregnancy and to provide insight in the physiological and pathological course of endothelial adaptation throughout human singleton pregnancies. During healthy pregnancy endothelial dependent vasodilatation measured by FMD and brachial artery diameter are increased. Women with a complicated pregnancy fell within the lower range of FMD values when compared to uncomplicated pregnancies, but as group, they did not differ from each other.

CHAPTER 3 is a review and meta-analysis with the purpose to systematically review and quantify current literature on kidney function during pregnancy, to estimate the extent of adaptation over the course of both physiological and hypertensive complicated singleton pregnancies and to determine a possible threshold defining normal. In healthy uncomplicated pregnancy kidney function is increased throughout gestation, reflected by an increased glomerular filtration rate (GFR) measured by inulin or creatinine clearance, and a decrease in serum creatinine. In contrast, this increased kidney function is not observed during hypertensive complicated pregnancy. Kidney function measured by creatinine clearances is less reliable during pregnancy compared to serum creatinine because of broad confidence intervals. Although the upper limit of the reference curve 
changes throughout gestation, from a clinical perspective, serum creatinine above 0.75 $\mathrm{mg} / \mathrm{dl}$ ( $66 \mathrm{umol} / \mathrm{L}$ ) should be considered abnormal during pregnancy.

CHAPTER 4 discusses an observational study wherein the initial systemic cardiovascular and renal adaptation was studied in women with a history of preeclampsia who either developed a recurrent hypertensive disorder in their next pregnancy or had an uneventful subsequent pregnancy. The initial maternal renal and hemodynamic adaptive response to pregnancy was attenuated in women with a hypertensive complicated pregnancy when compared to women who had an uneventful subsequent pregnancy. This study supports the concept that in women with a history of preeclampsia, recurrent hypertensive complication that develops in the next pregnancy is preceded by an abnormal initial renal and cardiovascular adaptive response to pregnancy.

CHAPTER 5 highlights an observational cohort study to estimate the prevalence of chronic kidney disease in women with a history of preeclampsia, based on the criteria written by the National Kidney Foundation. This foundation published the Kidney Disease: Improving Global Outcomes (KDIGO) guideline for the definition, classification, and prognosis of chronic kidney disease. Based on these criteria $14 \%$ of women with a history of preeclampsia have a decreased kidney function one year postpartum, compared to 10\% in the general population, mainly as a result of microalbuminuria. A yearly check-up of kidney function would be advisable for this subset of women.

CHAPTER 6 describes in a case-control study the relationship between vascular endothelial function and kidney function in women with a history of preeclampsia compared to women with uncomplicated pregnancies. Women with a history of preeclampsia have, five years after pregnancy, a decreased endothelial function expressed by FMD. There is however not a decrease in kidney function expressed by glomerular filtration rate and microalbuminuria, and no apparent correlation between FMD and kidney function. Even though decreased FMD and microalbuminuria both reflect endothelial dysfunction they may reflect different aspects of endothelial function. Alternatively the endothelial function as assessed by microalbuminuria may follow a different recovery rate than endothelial function expressed with FMD.

CHAPTER 7 discusses a cohort study, analysing the effects of aging on vascular function expressed by FMD and nitroglycerine induced dilation, particularly focussing on women with a history of preeclampsia compared to women with uncomplicated pregnancies. Arterial aging is a normal physiological process that develops gradually over time. One of the most clinically important changes in the development of vascular endothelial dysfunction is the impaired possibility to vasodilate. Nitroglycerine induced vasodilation decreased 
while brachial artery diameter increased over time in women with a history of vascular complicated and uncomplicated pregnancies suggesting stiffening of the vasculature. However, when corrected for confounders that are known to affect endothelial function, only the increase in diameter over time remained. Interestingly there was no change in FMD over time in both groups.

CHAPTER 8 encompasses a general discussion wherein wider implications and suggestions for future research are given.

CHAPTER 9 facilitates a valorisation addendum, indicating the value of this thesis for our society.

CHAPTER 10 provides a summary in English and Dutch. 



\section{SAMENVATTING}

Pre-eclampsie is een syndroom dat ontstaat tijdens de zwangerschap en is een uiting van endotheliale dysfunctie. Pre-eclampsie wordt gekenmerkt door hypertensie en proteïnurie en kan samengaan met uitgebreide intravasale stolling, lever- en nierproblemen. Om een ongecompliceerde maternale aanpassing aan de zwangerschap te bewerkstelligen zijn meerdere factoren van wezenlijk belang. Indien deze factoren onvoldoende aanwezig of zelfs afwezig zijn, dan is de maternale aanpassing gestoord en kan endotheliale dysfunctie ontstaan. Dit proefschrift geeft inzicht in de maternale aanpassingen aan de zwangerschap en beschrijft ook wat gebeurd indien dit proces abnormaal verloopt, ook wel maternale maladaptatie genoemd.

Lange termijn effecten van preeclampsie zijn een verhoogd risico op cardiovasculaire ziekte en chronische nierziekte. Dit proefschrift beschrijft tevens de lange termijn effecten van een door pre-eclampsie gecompliceerde zwangerschap op het vasculaire endotheel en de nierfunctie.

HOOFDSTUK 1 Algemene introductie en doelstellingen van dit proefschrift.

HOOFDSTUK 2 Review en meta-analyse waarin referentie curves weergegeven worden van flow mediated dilation (FMD) en de diameter van de brachiale arterie. Hierdoor is meer inzicht verkregen in de fysiologische en pathofysiologische veranderingen van het endotheel tijdens de zwangerschap. Tijdens een ongecompliceerde zwangerschap neemt de endotheel afhankelijke vasodilatatie gemeten door middel van FMD en de diameter van de brachiale arterie toe. Vrouwen met een door hypertensie gecompliceerde zwangerschap lieten lagere FMD waarden zien in vergelijking met vrouwen die een ongecompliceerde zwangerschap doormaakten.

HOOFDSTUK 3 Review en meta-analyse waarin de nierfunctie gedurende een fysiologische en hypertensief gecompliceerde zwangerschap gekwantificeerd wordt om een onderscheid te kunnen maken tussen een normale en abnormale adaptatie. Tijdens een ongecompliceerde zwangerschap neemt de maternale nierfunctie toe, en dit uit zich in een verhoogde glomerulaire filtratie (GFR) snelheid en een verlaagd serum creatinine. Tijdens een hypertensief gecompliceerde zwangerschap wordt een afgenomen nierfunctie waargenomen. Nierfunctie uitgedrukt in creatinineklaring lijkt minder toepasbaar tijdens de zwangerschap, gezien het grote betrouwbaarheidsinterval van de referentiecurve. De bovengrens van serum creatinine verandert gedurende de zwangerschap, maar vanuit een klinisch perspectief kan een waarde boven $0.75 \mathrm{mg} / \mathrm{dl}$ (66 umol/L) als abnormaal worden beschouwd. 
HOOFDSTUK 4 Observationele studie waarbij de vroege renale en cardiovasculaire adaptatie wordt onderzocht bij vrouwen die eerder een hypertensief gecompliceerde zwangerschap doormaakten en die wel of geen herhaling van de complicaties in een volgende zwangerschap kregen. De vroege maternale renale en hemodynamische adaptatie blijft verminderd in de groep vrouwen waarbij de volgende zwangerschap wederom gecompliceerd werd door een hypertensieve zwangerschap in vergelijking met vrouwen waarbij de volgende zwangerschap ongecompliceerd verloopt. Deze studie ondersteunt het concept dat vrouwen met een herhaaldelijk hypertensief gecompliceerde zwangerschap een initiële suboptimale en daarmee niet fysiologische renale en cardiovasculaire aanpassing aan een eventueel volgende zwangerschap hebben.

HOOFDSTUK 5 Observationele studie waarin vrouwen gemiddeld één jaar na een hypertensief gecompliceerde zwangerschap een verminderde nierfunctie laten zien conform de National Kidney Foundation (NKF) criteria. De NKF heeft een richtlijn gepubliceerd: Kidney Disease Improving Global Outcomes (KDIGO) waarin de definitie, classificatie en prognose van chronische nierziekte wordt beschreven. De richtlijn beschrijft het risico op het ontwikkelen van chronische nierziekte gebaseerd op nierfunctie uitgedrukt in een verlaagde glomerulaire filtratie snelheid en/of microalbuminurie. Op basis van de NKF criteria heeft 14\% van de vrouwen met een doorgemaakte pre-eclampsie een verminderde nierfunctie ten opzichte van $10 \%$ in de algemene populatie. Een jaarlijkse controle van de nierfunctie zou raadzaam zijn voor deze groep vrouwen.

HOOFDSTUK 6 Case-control studie die de relatie beschrijft tussen endotheel functie en nierfunctie bij vrouwen met en zonder een hypertensief gecompliceerde zwangerschap in de voorgeschiedenis. Vijf jaar na een doorgemaakte pre-eclampsie wordt bij deze vrouwen een verminderde endotheel-functie gemeten, uitgedrukt in een verlaagde FMD, maar geen vermindering in nierfunctie en geen correlatie tussen beide vertegenwoordigers van endotheel-functie. Hoewel een lage FMD en microalbuminurie allebei een reflectie zijn van endotheel dysfunctie, is het mogelijk dat beiden een ander aspect van de functie van het endotheel vertegenwoordigen. Een andere mogelijkheid is dat endotheel-functie uitgedrukt in microalbuminurie een ander herstelpatroon volgt dan endotheel-functie uitgedrukt in FMD.

HOOFDSTUK 7 Cohort studie waarin het effect van ouderdom op arteriële vasculaire functie beoordeeld bij vrouwen met en zonder een hypertensief gecompliceerde zwangerschap in de voorgeschiedenis. Arteriële vasculaire functie wordt uitgedrukt in FMD en nitroglycerine geïnduceerde dilatatie. Arteriële ouderdom is een normaal fysiologisch proces dat geleidelijk ontstaat. Met het toenemen van de leeftijd ontstaan verschillende arteriële veranderingen die bijdragen aan het verhoogde risico op cardiovasculaire 
aandoeningen. Een klinisch relevante verandering bij het ontwikkelen van endotheel dysfunctie is de verminderde dilatatie van een vat. Bij vrouwen met zowel een hypertensief gecompliceerde als ongecompliceerde zwangerschap verminderde de nitroglycerine geïnduceerde vasodilatatie, terwijl de diameter van de arteria brachialis toenam in de loop van de tijd. Dit suggereert een verstijving van de bloedvaten. Echter, wanneer gecorrigeerd werd voor factoren die zowel een bekend effect hebben op endotheel functie, als ook toenemen met de leeftijd, bleef enkel de toename in diameter aanwezig. Tussen beide groepen werd geen verschil in FMD, endotheel afhankelijke dilatatie, over de tijd gezien.

HOOFDSTUK 8 Algemene discussie waarin implicaties en suggesties voor vervolg onderzoeken worden gedaan.

HOOFDSTUK 9 Valorisatie appendix waarin de maatschappelijke waarde van dit proefschrift wordt beschreven.

HOOFDSTUK 10 Engelse en Nederlandse samenvatting. 


\title{
CHAPTER 11
}

\author{
List of co-authors \\ List of publications \\ Curriculum Vitae \\ Dankwoord
}




\section{LIST OF CO-AUTHORS}

Prof. dr. Hans-Peter Brunner LaRocca

Department of Cardiology, Maastricht University Medical Centre, the Netherlands

Dr. Tom Cornelis

Department of Nephrology, Jessa Hospital, Hasselt, Belgium

Dr. Joris van Drongelen

Department of Obstetrics and Gynaecology, Radboud University Nijmegen Medical

Centre, the Netherlands

Dr. Vanessa van Empel

Department of Cardiology, Maastricht University Medical Centre, the Netherlands

Drs. Tessa A.G. van Gansewinkel

Department of Obstetrics and Gynaecology, Maastricht University Medical Centre, the Netherlands

Dr. Chahinda Ghossein-Doha

Department of Obstetrics and Gynaecology, Maastricht University Medical Centre, the Netherlands \& Department of Cardiology, Maastricht University Medical Centre, the Netherlands

Drs. Sander de Haas

Department of Obstetrics and Gynaecology, Maastricht University Medical Centre, the Netherlands

Drs. Wieteke M. Heidema

Department of Obstetrics and Gynaecology, Radboud University Nijmegen Medical

Centre, the Netherlands

Prof. dr. Ir. Arnold P. Hoeks

Department of Biomedical Engineering, Maastricht University, the Netherlands

Prof. dr. Abraham A. Kroon

Department of Internal Medicine, Maastricht University Medical Centre the Netherlands

Dr. Sander M.J. van Kuijk

Department of Clinical Epidemiology and Medical Technology Assessment, Maastricht University Medical Centre, the Netherlands

Ir. Erjona Morina-Shijaku

Department of Obstetrics and Gynaecology, Maastricht University Medical Centre, the Netherlands

Drs. Eva G. Mulder

Department of Obstetrics and Gynaecology, Maastricht University Medical Centre, the Netherlands 
Prof. dr. Gerard Pasterkamp

Department of Experimental Cardiology Laboratory, University Medical Centre, Utrecht, the Netherlands

Dr. Louis L.H. Peeters

Department of Obstetrics and Gynaecology, Maastricht University Medical Centre, the Netherlands

Dr. Ir. Koen D. Reesink

Department of Biomedical Engineering, Maastricht University, the Netherlands

Dr. Ir. Hester M. den Ruijter

Department of Experimental Cardiology Laboratory, University Medical Centre, Utrecht, the Netherlands

Dr. Ralph R. Scholten

Department of Obstetrics and Gynaecology, Radboud University Nijmegen Medical Centre, the Netherlands

Dr. Julia J. Spaan

Department of Obstetrics and Gynaecology, Maastricht University Medical Centre, the Netherlands

Prof. dr. Marc E.A. Spaanderman

Department of Obstetrics and Gynaecology, Maastricht University Medical Centre, the Netherlands

Prof. dr. Anton Jan van Zonneveld

Department of Experimental Cardiology Laboratory, University Medical Centre, Utrecht, the Netherlands 


\section{LIST OF PUBLICATIONS}

Early pregnancy circulatory adaptation and recurrent hypertensive disease; an explorative study. Veronica A. Lopes van Balen, Julia J. Spaan, Chahinda Ghossein-Doha, Sander M.J. van Kuijk, Marc. E.A. Spaanderman and Louis L.H Peeters.

Reproductive Science 2013 20(9):1069-74.

The prevalence of chronic kidney disease after preeclampsia. Veronica A. Lopes van Balen, Julia J. Spaan, Tom Cornelis and Marc E.A. Spaanderman.

Journal of Nephrology 201730 (3):403-409.

Endothelial and kidney function in women with a history of preeclampsia and healthy parous controls. Veronica A. Lopes van Balen, Julia J. Spaan, Tom Cornelis, Koen D. Reesink, Wieteke M. Heidema, Ralph R. Scholten and Marc E.A. Spaanderman.

Microvascular Research 2018 116:71-76

Physiologic adaptation of endothelial function to pregnancy: a systematic review and metaanalysis. Veronica A. Lopes van Balen, Tessa A.G. van Gansewinkel, Sander de Haas, Chahinda Ghossein-Doha, Sander M.J. van Kuijk, Joris van Drongelen and Marc E.A. Spaanderman.

Ultrasound Obstetrics and Gynecology 2017 50(6):697-708

Vascular ageing in young and middle-aged women after hypertensive complicated and uncomplicated pregnancy. Veronica A. Lopes van Balen, Chahinda Ghossein-Doha, Julia J. Spaan, Eva G. Mulder, Sander M.J. van Kuijk, Erjona-Morina Shijaku, Hester M. den Ruijter, Gerard Pasterkamp, Anton Jan van Zonneveld, Abraham A. Kroon, Vanessa van Empel, Hans-Peter Brunner LaRocca, Arnold P. Hoeks, Koen D. Reesink and Marc E.A. Spaanderman.

[Submitted]

Kidney function during pregnancy: a systematic review and meta-analysis. Veronica A. Lopes van Balen, Tessa A.G. van Gansewinkel, Sander de Haas, Julia J. Spaan, Tom Cornelis, Chahinda Ghossein-Doha, Sander M.J. van Kuijk, Joris van Drongelen and Marc E.A. Spaanderman.

[Accepted for publication in Ultrasound Obstetrics and Gynecology]

Metabolic syndrome as a risk factor for hypertension after preeclampsia. Julia J. Spaan, Simone J. Sep, Veronica A. Lopes van Balen, Marc E.A. Spaanderman, and Louis L.H. Peeters.

Obstetrics and Gynaecology 2012 Aug 120(2 Pt 1):311-7 
HOMA beta cell function as a predictor of abnormal oral glucose tolerance testing in pregnancy. Jonas Ellerbrock, Jolijn M.H.A Bohnen, Veronica A. Lopes van Balen, Eva G. Mulder, Robert Aardenburg and Marc E.A. Spaanderman.

Gynaecological Endocrinology 2017 33(12):911-917

Agreement Conform Current Operational Rules and Directives (ACCORD), a method to reach multidisciplinary consensus in healthcare. Stéphanie M.P. Lemmens, Hubertina C.J. Scheepers, Veronica A. Lopes van Balen, Yvonne C.M. Röselaers, Raymond de Vries and Marc E.A. Spaanderman.

[Submitted] 



\section{CURRICULUM VITAE}

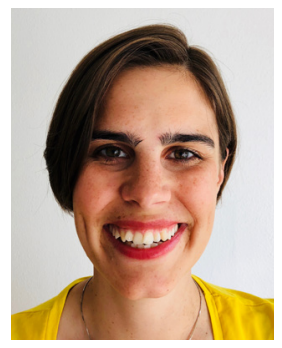

Veronica werd geboren op 23 februari 1987 in Rio de Janeiro, Brazilië, dochter van een Braziliaanse moeder en een Nederlandse vader. Ze behaalde haar VWO diploma op het Mollerlyceum te Bergen op Zoom in 2005 en startte vervolgens de opleiding geneeskunde aan de Universiteit van Maastricht.

Na haar afstuderen werkte Veronica tussen 2012 en 2014 als arts niet in opleiding tot specialist (ANIOS) bij de afdeling obstetrie en gynaecologie in het Sint Elisabeth Ziekenhuis te Tilburg en in het Erasmus Universitair Medisch Centrum te Rotterdam.

In 2015 startte ze haar promotietraject onder begeleiding van Prof. dr. Marc E.A. Spaanderman en dr. Julia J. Spaan waarvan het resultaat hier voor u ligt. Tijdens deze periode heeft ze als ANIOS en onderzoeker gewerkt in het Maastricht Universitair Medisch Centrum. 


\section{DANKWOORD}

Dit boekje leek ver weg toen ik vier jaar geleden aan mijn promotie begon, maar de tijd is voorbijgevlogen. Ik ben blij en trots dat het nu af is.

Dit proefschrift was er niet gekomen zonder jullie steun. Vele mensen hebben mij gesteund de afgelopen jaren, waarvan ik een aantal in het bijzonder wil bedanken.

Om te beginnen gaat mijn dank uit naar alle vrouwen die vrijwillig hebben deelgenomen aan de studies in dit proefschrift.

Mijn promotor prof. dr. Marc Spaanderman en copromotor dr. Julia Spaan.

Beste Marc, bedankt dat je me deze kans hebt gegeven. Ik waardeer je adviezen en ben dankbaar voor de steun die je me in de loop der jaren op zowel persoonlijk als professioneel vlak hebt gegeven. Door jou heb ik geleerd om van alles een leermoment te maken. Je hebt me het vertrouwen gegeven om verantwoordelijkheid te nemen in verschillende situaties, waardoor ik kon groeien en zoveel nieuws kon leren.

Beste Julia, bedankt dat je mijn copromotor wilde zijn. Je enthousiasme voor onderzoek, je steun, je kritische en nuchtere blik hebben me door uitdagende periodes heen geholpen. Ik ben zo blij dat je een plek in Breda hebt gevonden.

Beoordelingscommissie prof. dr. B.W.W. Kramer, prof. dr. J.P. Kooman, prof. dr. W. Gyselears, prof. dr. M.T.E. Hopman. Ik dank u allen voor het lezen en beoordelen van mijn proefschrift en ik dank de promotiecommissie voor de oppositie tijdens mijn promotie.

Stafleden, arts-assistenten en secretariaat van het MUMC+, veel dank voor de prettige samenwerking.

Alle medeauteurs, bedankt voor jullie kritische opmerkingen en fijne suggesties. Sander van Kuijk, het was heel fijn om jou als statistisch adviseur te hebben. Je geduldige en prettige manier van uitleg geven zijn verhelderend.

Heren van de Biomedische Technologie, Koen en Arnold, het was heel fijn dat jullie mij wilden helpen. Na onze gesprekken was ik altijd weer enthousiast en geïnspireerd om verder te gaan met mijn onderzoek.

Alle dames van het Transmuraal Vrouwen Dagcentrum in het MUMC+,

Nathalie, Marie-Jeanne, Lonneke, Gonny, Betul, Stella, Monique, Caroline, Yvonne en Yolanda. Bedankt voor de samenwerking de afgelopen jaren. Ik heb met veel plezier met jullie samengewerkt. Het waren leuke en leerzame jaren. Ik ben heel trots op jullie en het werk dat we op het TVDC doen.

\section{TVDC doc's Eva en Veronique}

Eef, het voelt alsof we samen het TVDC hebben gemaakt tot wat het nu is en daar ben ik heel trots op. Je open en intrinsiek vrolijke houding kan ik erg waarderen. Omdenken is een kunst! 
V2, bedankt dat je het team bent komen versterken. Je bent een prettige en enthousiaste collega en een superfijne kamergenoot.

Eri, ik ben blij dat je onze TVDC vaatfunctielaborante bent. Je geduldige manier van werken en oog voor detail zijn enkele eigenschappen van je die ik waardeer. Bedankt voor de samenwerking de afgelopen jaren.

Tessa, bedankt dat je tijdens je geneeskundestudie en in je vrije tijd wilde helpen met mijn onderzoek. Het was een kers op de taart dat we samen naar een congres konden gaan. Door een kleine vakantie eraan vast te plakken hebben we vijf nationale parken in de VS gezien. Hoe dan? Door 40000 km te rijden in één week :-). Wat een avontuur!

Ghislaine, oud kamergenootje, het waren fijne tijden samen op de UNS 50 en ik ben heel blij dat je nu met manlief en prachtige dochter Amélie samen bent.

Paranimfen Stephanie en Jonas, ik waardeer het enorm dat jullie naast mij staan.

Steef, het was heerlijk om samen de dag te beginnen met een kopje koffie. Samen op de bank, raampje open, zonnestralen in de kamer. Fijne momenten.

Jonas, Sugardaddy, samen nadenken over hoe iets te verwoorden in je artikelen waren hoogtepunten van mijn week. Dat we dat nog vaak samen kunnen doen.

Erwin, Manouk, Marian, studiemaatjes, bedankt voor alle fijne momenten die we samen hebben gehad. ledereen heeft inmiddels zijn plekje gevonden. Nu ik nog (;)

Maarten, ik ben dankbaar dat ik je mijn broer kan noemen en dat je een luisterend oor had gedurende alle momenten dat ik even wat minder enthousiast was.

Wolfpack, Tom \& Kelly, ik ben zo blij dat ik jullie heb leren kennen. Onze weekenden samen zijn kostbaar. Dat we nog vaak samen op vakantie kunnen gaan :)

Jos en Jantine, bedankt voor jullie steun de afgelopen jaren. Ik vind het bijzonder fijn dat ik bij de familie Vriens hoor en dat jullie mij accepteren zoals ik ben.

$\mathrm{Pa}$, bedankt dat je altijd achter mijn beslissingen staat en dat je me de ruimte hebt gegeven om mijn weg te vinden.

Mae, minha amiga querida. A maravilhosa mulher que me fez a mulher que eu sou hoje. Tenho certeza absoluta que voce seria trots como um pauw durante a minha defesa. Vou sentir sempre falta de você.

Dennis, mijn tweede ik, mijn maatje, liefde van mijn leven. Wat zal ik intens gelukkig zijn als we weer echt samenwonen na al die jaren op en neer pendelen van Roosendaal naar Maastricht. 
